\title{
Feedforward and Feedback Tracking Control of Diffusion-Convection-Reaction Systems using Summability Methods
}

\author{
Von der Fakultät Maschinenbau der Universität Stuttgart \\ zur Erlangung der Würde eines \\ Doktor-Ingenieurs (Dr.-Ing.) genehmigte Abhandlung
}

Vorgelegt von

Thomas Meurer

geboren in Gaildorf

Hauptberichter: $\quad$ Prof. Dr.-Ing. Dr.h.c. M. Zeitz

Mitberichter: Prof. Dr. P. Rouchon

Tag der mündlichen Prüfung: 24. Juni 2005

Institut für Systemdynamik und Regelungstechnik der Universität Stuttgart 



\section{Vorwort}

Die vorliegende Arbeit entstand in den Jahren 2001 bis 2005 während meiner Tätigkeit als wissenschaftlicher Mitarbeiter am Institut für Systemdynamik und Regelungstechnik der Universität Stuttgart. Die durchgeführten Untersuchungen wurden u.a. von der Deutschen Forschungsgemeinschaft (DFG) im Rahmen des Projekts "Flachheitsbasierte Regelung von Systemen mit verteilten Parametern" gefördert.

Mein besonderer Dank gilt Herrn Prof. Dr.-Ing. Dr.h.c. M. Zeitz für die ausgezeichnete und engagierte Betreuung bei der Durchführung der Arbeit. Ihm gilt auch mein herzlicher Dank für die stetige und langjährige Förderung seit der Studienzeit, sein allzeit "offenes Ohr" sowie die eingeräumten fachlichen Freiräume.

Sehr gefreut habe ich mich über die Übernahme des Mitberichts durch Herrn Prof. Dr. P. Rouchon von der École des Mines de Paris, einem der Mitbegründer der Theorie der flachen Systeme und deren Erweiterung auf den verteilt-parametrischen Fall. Ihm danke ich für sein Interesse, die schnelle Durchsicht des Manuskripts sowie seine konstruktiven Hinweise.

Ausserdem bedanke ich mich bei dem Institutsleiter, Herrn Prof. Dr.-Ing. Dr.h.c.mult. E.D. Gilles, für die günstigen Arbeitsbedingungen in seinem Institut. Den Kolleginnen und Kollegen am Institut danke ich für das gute Arbeitsklima und stete Hilfsbereitschaft. Besonders danke ich Matthias Bitzer, Knut Graichen und Reto Köhler für die sehr gute Zusammenarbeit in der Gruppe von Herrn Prof. Zeitz. Darüberhinaus bedanke ich mich bei Jens Becker, Matthias Boldt, Alexander Schaum, Michael Schenk und ganz speziell bei Marc Oliver Wagner, die durch ihre Studien- und Diplomarbeiten wertvolle Beiträge zu der vorliegenden Thematik geleistet haben.

Schliesslich, aber nicht zuletzt, danke ich meinen Eltern für ihre Unterstützung und Barbara für ihren Rückhalt und ihr Verständnis. 
Für meine Eltern 


\section{Contents}

List of Symbols

VIII

$\begin{array}{lll}\text { Abstract } & \text { XI }\end{array}$

German summary / Deutsche Kurzfassung $\quad$ XIII

1 Introduction $\quad 1$

1.1 Control of systems governed by PDEs . . . . . . . . . . . . . . . . 1

1.2 Flatness-based methods for PDEs . . . . . . . . . . . . . . . . . 2

1.3 Goals of the thesis . . . . . . . . . . . . . . . . . 4

1.4 Structure of the thesis . . . . . . . . . . . . . . . . . . 6

2 Formal power series parameterization of boundary controlled DCR-systems 8

2.1 Scalar DCR-equations with boundary input . . . . . . . . . . . 8

2.1.1 The linear heat equation . . . . . . . . . . . . . . . 9

2.1.2 The linear diffusion-convection equation . . . . . . . . . . . . 14

2.1.3 Scalar DCR-equations with polynomial nonlinearities . . . . . . . . . . 17

2.2 MIMO DCR-equations with boundary inputs . . . . . . . . . . . . 20

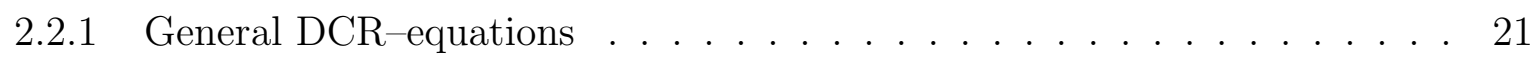

2.2.2 A tubular reactor model . . . . . . . . . . . . . . . . 25

2.3 Formal power series parameterizability, flatness and controllability of DCR-systems 30

2.4 Conclusions . . . . . . . . . . . . . . . . . . . . 33

3 Summability methods and sequence transformations $\quad 34$

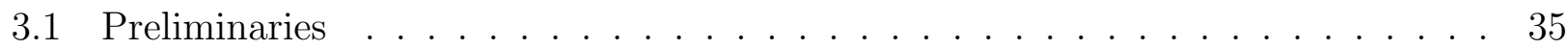

3.2 Asymptotic power series and Gevrey functions . . . . . . . . . . . . . 37

3.2.1 Formal power series on Banach spaces . . . . . . . . . . . . . 37 
3.2 .2 Asymptotic expansions . . . . . . . . . . . . . . . . . 39

3.2 .3 Gevrey asymptotics . . . . . . . . . . . . . . . . 41

3.3 Summation of power series . . . . . . . . . . . . . . . . . . 42

3.3.1 General considerations . . . . . . . . . . . . . . . 43

3.3.2 Summation in a direction . . . . . . . . . . . . . . . . 44

3.4 Towards numerical implementation and convergence acceleration . . . . . . . . 48

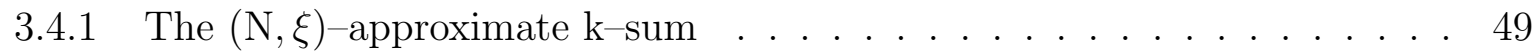

3.4 .2 Numerical example: the divergent Euler series . . . . . . . . . . . . . 51

3.5 General sequence transformations . . . . . . . . . . . . . . . . 53

3.5.1 General considerations . . . . . . . . . . . . . 53

3.5.2 Examples of linear and nonlinear sequence transformations . . . . . . . . 54

3.5.3 Numerical example: the divergent Euler series . . . . . . . . . . . . 56

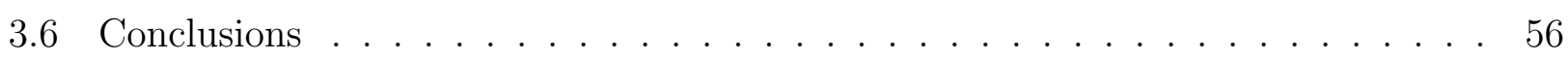

4 Motion planning and feedforward control $\quad 58$

4.1 Enhanced motion planning in Gevrey classes . . . . . . . . . . . . . . . . . 59

4.1.1 Multiple transitions between stationary profiles . . . . . . . . . . . . 59

4.1.2 Multiple transitions between arbitrary profiles . . . . . . . . . . 61

4.2 Power series solutions and summability methods for PDEs . . . . . . . . . . 63

4.3 Scalar DCR-equations with boundary input . . . . . . . . . . . . . 64

4.3.1 Enhanced motion planning for the linear heat equation . . . . . . . . . 64

4.3.2 Convergence acceleration for the linear diffusion-convection equation . . 68

4.3 .3 Summary . . . . . . . . . . . . . . . . . . 72

4.4 MIMO DCR-equations with boundary inputs . . . . . . . . . . . 73

4.4.1 Feedforward control design for general DCR-systems . . . . . . . . 73

4.4.1.1 Summation of series resulting from DCR-systems . . . . . . . 73

4.4.1.2 Choice of summation parameters in $(\mathrm{N}, \xi)$-approximate k-sum . 75

4.4.2 Feedforward control design for the tubular reactor model . . . . . . . . 76

4.5 Conclusions . . . . . . . . . . . . . . . . . . . . . . . 79

$5 \quad$ Flatness-based feedback boundary tracking control of DCR-systems 82

5.1 Scalar DCR-equations with boundary input . . . . . . . . . . . . 82

5.1.1 Feedback tracking control design for the linear heat equation . . . . . . . 83 
5.1.1.1 Finite-dimensional design model via formal power series and summation methods . . . . . . . . . . . . . 83

5.1.1.2 Tracking control with observer . . . . . . . . . . . 87

5.1.1.3 Stability of the tracking control scheme . . . . . . . . . . 89

5.1.1.4 Simulation results . . . . . . . . . . . . . . . . . 92

5.1 .2 Nonlinear scalar DCR-equations . . . . . . . . . . . . . . 92

5.2 MIMO DCR-equations with boundary inputs . . . . . . . . . . . . . 93

5.2.1 Feedback tracking control design for general DCR-systems . . . . . . . . 93

5.2.1.1 Finite-dimensional design model via formal power series and summation methods . . . . . . . . . . . . . 93

5.2.1.2 Flatness-based tracking control with observer . . . . . . . . 96

5.2.1.3 Spatial profile estimation . . . . . . . . . . . . . 99

5.2.1.4 The case $\mathrm{M}<\mathrm{K}$. . . . . . . . . . . . . . . 100

5.2.2 Feedback control for the tubular reactor model . . . . . . . . . . . . . 100

5.2.2.1 Nonlinear controller normal form . . . . . . . . . . . . 101

5.2.2.2 Simulation results for flatness-based tracking control with ob-

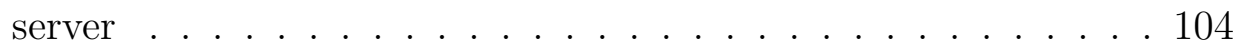

5.2.2.3 Simulation results for feedforward control and output feedback . 106

5.3 Conclusions . . . . . . . . . . . . . . . . . . . . . . 110

6 Conclusions and outlook $\quad 111$

$\begin{array}{ll}\text { A Mathematical glossary } & 113\end{array}$

A.1 Analysis . . . . . . . . . . . . . . . . . . . 113

A.2 Complex analysis . . . . . . . . . . . . . . . . . . . 114

$\begin{array}{ll}\text { B Smooth functions with compact support } & 115\end{array}$

C An algorithm for the efficient evaluation of nonlinear differential recurrence $\begin{array}{ll}\text { relations } & 117\end{array}$

D Lemmas and parameters for the non-isothermal tubular reactor model 119

D.1 Lemma on recursion . . . . . . . . . . . . . . . . . . . . 119

D.2 Model and simulation parameters . . . . . . . . . . . . . . 120

$\begin{array}{ll}\text { Bibliography } & 121\end{array}$

$\begin{array}{lr}\text { Internal reports, student and diploma theses } & 128\end{array}$ 


\section{List of Symbols}

The following list only contains symbols that are used continuously throughout the text. Local symbols are not listed.

\section{Variables}

$\boldsymbol{\zeta}(t) \quad$ vector of time-variable functions

$u(t) \quad$ scalar input from 1 -dimensional functional space $\mathcal{U}$

$u_{d}(t) \quad$ feedforward boundary control

$\boldsymbol{u}(t) \quad$ input vector from $M$-dimensional functional space $\mathcal{U}$

$\boldsymbol{u}_{d}(t) \quad M$-vector of feedforward boundary controls

$\boldsymbol{u}_{S} \quad M$-vector of stationary input values

$x(z, t) \quad$ state from 1-dimensional functional space $\mathcal{X}$

$x_{d}(z, t) \quad$ desired state profile

$\hat{x}(z, t) \quad$ formal power series $\hat{x}(z, t)=\sum_{n=0}^{\infty} \hat{x}_{n}(t) z^{n}$

$\underline{\hat{x}}_{n}(t) \quad \underline{\hat{x}}_{n}(t)=\left[\hat{x}_{0}(t), \hat{x}_{1}(t), \ldots, \hat{x}_{n}(t)\right]^{T}$ for $n \in \mathbb{N}_{0}$

$\boldsymbol{x}(z, t) \quad$ state vector from $K$-dimensional functional space $\mathcal{X}[0,1]$

$\boldsymbol{x}_{d}(z, t) \quad K$-vector of desired state profiles

$\hat{\boldsymbol{x}}(z, t) \quad$ formal power series $\hat{\boldsymbol{x}}(z, t)=\sum_{n=0}^{\infty} \hat{\boldsymbol{x}}_{n}(t) z^{n}$

$\hat{\boldsymbol{x}}_{2 N-2}^{\prime} \quad$ state vector $\hat{\boldsymbol{x}}_{2 N-2}^{\prime}=\left[x_{0}, x_{2}, \ldots, x_{2 N-2}\right]^{T}$ of the SISO design model in series coefficients

$\underline{\hat{\boldsymbol{x}}}_{n}(t) \quad \underline{\hat{\boldsymbol{x}}}_{n}(t)=\left[\hat{\boldsymbol{x}}_{1}^{T}(t), \hat{\boldsymbol{x}}_{2}^{T}(t), \ldots, \hat{\boldsymbol{x}}_{n}^{T}(t)\right]^{T}$ for $n \in \mathbb{N}_{0}$

$\underline{\hat{\boldsymbol{x}}}_{2 N-2}^{\prime} \quad$ state vector $\underline{\hat{\boldsymbol{x}}}_{2 N-2}^{\prime}=\left[\hat{\boldsymbol{x}}_{0}^{T}, \hat{\boldsymbol{x}}_{2}^{T}, \ldots, \hat{\boldsymbol{x}}_{2 N-2}^{T}\right]^{T}$ of the MIMO design model in series coefficients 
$\boldsymbol{x}_{S}(z) \quad K$-vector of stationary profiles

$y(t) \quad$ scalar parameterizing function

$y_{d}(t) \quad$ desired trajectory for parameterizing function $y(t)$

$\boldsymbol{y}(t) \quad M$-vector of parameterizing functions

$\boldsymbol{y}_{d}(t) \quad M$-vector of desired trajectories for parameterizing function $\boldsymbol{y}(t)$

$\boldsymbol{y}_{S} \quad M$-vector of stationary values of the parameterizing function

\section{Scalars}

$K \quad$ dimension of state vector $\boldsymbol{x}(z, t)$

$M \quad$ dimension of input vector $\boldsymbol{u}(z, t)$

$p_{i, N+1} \quad$ parameters for the dynamics of the tracking error

$q_{i, N+1} \quad$ parameters for the dynamics of the observer error

\section{Formal power series and summation methods}

$\boldsymbol{A}(\Omega, \mathbb{E}) \quad$ set of all functions $x \in \boldsymbol{H}(\Omega, \mathbb{E})$ having asymptotic expansion $\hat{x}(z)$

$\mathcal{A I}_{p_{\mathcal{A I}}}^{\left(q_{\mathcal{A I}}\right)}(z)$ Aitken's $\Delta^{2}$-formula

$\boldsymbol{A}^{(k)}(S, \mathbb{E})$ set of all functions $x$, holomorphic, of exponential growth at most $k$ in $S$, and continuous at the origin

$\boldsymbol{A}_{\alpha}(\Omega, \mathbb{E}) \quad$ set of all functions $x \in \boldsymbol{H}(\Omega, \mathbb{E})$ having asymptotic expansion $\hat{x}(z)$ of order $\alpha$

$\mathbb{E} \quad$ Banach space equipped with a norm $\|\cdot\|$ over the field of complex numbers $\mathbb{C}$

$\mathbb{E}\{z\}_{k, d} \quad$ set of all $k$-summable power series $\hat{x}(z)$ in direction $d$

$\mathbb{E}[[z]] \quad$ set of all formal power series $\hat{x}$ in $z \in \mathbb{C}$

$\mathbb{E}[[z]]_{\alpha} \quad$ set of all formal power series $\hat{x}$ in $z \in \mathbb{C}$ of Gevrey order $\alpha \geq 1$

$\mathbb{E}\{z\} \quad$ set of all convergent power series $\hat{x}$ in $z \in \mathbb{C}$

$G_{M, R, \alpha}(\Omega)$ Gevrey class of order $\alpha$ in $\Omega, M, R$ positive constants

$\boldsymbol{H}(\Omega, \mathbb{E}) \quad$ set of all $\mathbb{E}$-valued functions holomorphic in a sectorial region $\Omega$

$\mathcal{L}_{k} \quad$ Laplace integral of order $k$

$\mathcal{S}_{P} \quad$ partial summation 
$\mathcal{S}_{k, d} \quad k$-summation in direction $d$

$\mathcal{S}_{k}^{N, \xi} \quad(N, \xi)$-approximate $k$-summation

$\delta_{p_{\delta}}^{\left(q_{\delta}\right)}(\zeta) \quad$ Weniger's $\delta$-algorithm

$\hat{x}(z) \quad$ formal power series (in $z \in \mathbb{C}$ ) with coefficients $\left\{\hat{x}_{n}\right\}_{n}^{\infty}$ from a Banach space $\mathbb{E}$

\section{Abbreviations}

Da Damköhler number

Le $\quad$ Lewis number

Pe Peclet number

2DOF two-degree-of-freedom

BC boundary condition

BVP boundary value problem

CAS computer-algebra-system

DCR diffusion-convection-reaction

DCRE diffusion-convection-reaction equation

DPS distributed parameter system

FPSP formal power series parameterizability

GST generalized sequence transformation

IBVP initial-boundary-value problem

IC initial condition

IVP initial-value problem

MIMO multiple input multiple output

MOL method--of-lines

ODE ordinary differential equation

PDE partial differential equation

SISO single input single output

$\mathbb{I}_{K} \quad K \times K$ unit matrix

$\mathrm{O}_{K} \quad K \times K$ zero matrix 


\section{Abstract}

Diffusion-convection-reaction (DCR) processes occur in a large variety in chemical and biochemical engineering such as fixed-bed tubular reactors for production or degradation. These systems typically exhibit complex dynamical behavior, which in particular complicates control design. Thereby, advanced control strategies are required due to the increasing demands on product quality and production efficiency.

Since modeling of these processes usually leads to distributed-parameter systems (DPSs), control design is either based on early or late lumping approaches. In the early lumping approach, the system is approximated first and control design is performed based on the lumped model. This often leads to high-dimensional and complex feedback control structures. On the other hand in the late lumping approach, control synthesis is based on the infinite-dimensional process model. Although theoretically appealing, this approach may lead to non-implementable control laws and is mainly restricted to linear systems.

Furthermore, classical early and late lumping approaches typically address stabilization while neglecting the tracking control problem. For its solution, differential flatness is a well-established tool for finite-dimensional nonlinear systems with recent extensions to motion planning and feedforward control design for infinite-dimensional systems governed by partial differential equations (PDEs). Here, the property of parameterizability can be identified as the constitutive principle with the type of PDE determining the parameterization approach. In particular for linear and certain nonlinear DCR-equations with boundary inputs, power series in the spatial coordinate are applied to determine differential recursions for the time-variable series coefficients. The solution of these recursions can be expressed in terms of a parameterizing function (corresponding to the flat output) and its time-derivatives up to infinite order.

Once this parameterization is obtained, the respective feedforward controls, i.e. the boundary input which ensures tracking of an appropriate trajectory for the parameterizing function, follows directly from the evaluation of the inhomogeneous boundary conditions. Nevertheless, the necessary proof of uniform convergence is directly related to the problem of motion planning. Thereby several drawbacks emerge. At first, uniform convergence restricts motion planning to certain smooth functions having compact support. Secondly, no information on the respective speed of convergence, which e.g. might be slow for convection dominated systems, can be extracted from the convergence proof. Finally for nonlinear problems, the resulting convergence conditions relate motion planning and process parameters, which further constraints the applicability of the approach. 
In order to overcome these limitations, this thesis considers a combination of formal power series and suitable summation methods, whereby the notion formal denotes the fact that the radius of convergence might well be equal to zero. This is in particular focused on enhanced motion planning and enlarged applicability of the feedforward control design using formal power series.

Therefore, the notion of formal power series parameterizability (FPSP) is introduced, which formally allows to overcome the restriction to uniformly convergent power series. The underlying algebraic structure of the considered space of formal power series is thereby determined by summability methods with the focus on $k$-summability. This in particular allows to deal with both uniformly convergent as well as certain divergent series. Although various results on $k^{-}$ summability of formal solutions to PDEs are available, the theory is far from complete and is restricted to the Cauchy problem on unbounded domains. Furthermore, nonlinear problems typically allow only the determination of a finite number of coefficients for the formal series.

Hence, certain modifications of the summability approach are required which lead to the consideration of generalized sequence transformations (GSTs). These methods provide highly accurate approximations of the sum of a given slowly converging or possibly diverging series based on only a finite number of series coefficients. Within this framework, a variant of $k$-summation is introduced, namely the $(N, \xi)$-approximate $k$-summation, which approximately combines the advantages of $k$-summation with the demands imposed from practical problems. This novel combination of FPSP and GSTs greatly extends the applicability of the formal power series approach for feedforward tracking control design, as is illustrated in various examples including divergent solutions to the linear heat equation, slowly converging series in case of the linear diffusion-convection equation, and the nonlinear model of a tubular reactor within several branches of operation.

Since pure feedforward control is only applicable for the nominal case with perfectly known and stable plant, feedback control is required to account for instability, model errors, and/or exogenous disturbances. Therefore it is shown, that a re-interpretation of FPSP allows to determine a finite-dimensional inherently flat approximation of the governing infinite-dimensional DPS. This in particular allows to adopt standard techniques from flatness-based tracking control design with observer. In addition, it is shown that the estimated data from the observer can be utilized for spatial profile estimation e.g. for monitoring purposes. Thereby, the range of applicability of this feedback control approach can be increased by considering the $(N, \xi)-$ approximate $k$-summation, which is illustrated in numerical simulations for tracking control of the linear heat equation and the nonlinear model of a non-isothermal tubular reactor.

In summary, formal power series in conjunction with sophisticated summation methods provide a systematic analysis and design approach suitable for numerical evaluation and computeraided implementation for certain practically relevant nonlinear parabolic systems of second order PDEs with boundary inputs. 


\section{Deutsche Kurzfassung}

\section{Steuerung und Folgeregelung von Diffusions-Konvektions-Reaktions- Systemen unter Verwendung von Summationsmethoden}

Systeme, die durch Diffusions-Konvektions-Reaktions-Gleichungen (DKRGn) beschrieben werden, treten in großer Vielfalt beispielsweise im Bereich der Verfahrenstechnik auf. Typische Modellprozesse umfassen Rohr- und Festbettreaktoren mit komplexem dynamischen Verhalten. Die Analyse dieser parabolischen verteilt-parametrischen Systeme wird durch deren unendlichdimensionalen Charakter bestimmt, der sich in den entsprechenden Methoden der reinen, angewandten und numerischen Mathematik widerspiegelt. Aus Ingenieurssicht gilt im Allgemeinen das spezielle Interesse der Untersuchung des dynamischen Eingangs-/Ausgangsverhaltens. Andererseits und wie auch in dieser Arbeit gezeigt wird, eröffnet die Bestimmung des inversen Systems weiterführende Einsichten in die Systemdynamik, die zum modellbasierten Entwurf von Steuerungen und Regelungen genutzt werden können.

Im Allgemeinen beruht der modellbasierte Regelungsentwurf für Systeme, die durch partielle Differenzialgleichungen (PDGLn) beschrieben werden, auf zwei Konzepten:

- Entwurf einer Regelung ggf. mit Beobachter basierend auf einer geeigneten Approximation der Modellgleichungen ("early lumping") beispielsweise mittels Differenzenverfahren, Finite-Element-Methoden, modalen Ansätzen (Georgakis et al., 1977a,b,c; Balas, 1978) oder Projektionsverfahren (Ray, 1981; Atwell and King, 2001; Christofides, 2001).

- Entwurf einer Regelung ggf. mit Beobachter direkt anhand der verteilt-parametrischen Modelle ("late lumping") mittels funktionalanalytischer Methoden (Fattorini, 1968; Nambu, 1979, 1984; Lasiecka and Triggiani, 1983; Curtain and Zwart, 1995) und anschliessede Approximation der unendlich-dimensionalen Regelung.

Beide Ansätze weisen gewisse Nachteile auf. Im Fall des "early lumping" stellt sich insbesondere die Frage nach der Konvergenz, d.h. strebt die Lösung des reduzierten Modells gegen 
die Lösung des Originalmodells. Hieraus ergeben sich meist hoch-dimensionale Regelgesetze, die die Anwendbarkeit deutlich einschränken. Andererseits muss der mathematisch exakte, im Allgemeinen auf unendlich-dimensionale Regelgesetze führende "late lumping" Entwurf zur Realisierung und Implementierung geeignet approximiert werden. Weiterhin ist diese Methodik mit wenigen Ausnahmen auf lineare Systeme beschränkt.

Insbesondere ist zu bemerken, dass mit Ausnahme der Arbeiten zur optimalen Steuerung und Regelung (Butkovsky, 1969; Lions, 1971; Ray, 1981; Fursikov, 1999), deren Ergebnisse jedoch mit einem hohen numerischen Aufwand einhergehen, meist die Stabilisierungsaufgabe jedoch nicht das Trajektorien-Folgeproblem betrachtet wird. Zu dessen Lösung hat sich bei nichtlinearen endlich-dimensionalen Systemen die Eigenschaft der differenziellen Flachheit als eine geeignete Basis zur Trajektorienplanung sowie zum systematischen Entwurf von Steuerungen und Folgeregelungen mit Beobachter erwiesen (Fliess et al., 1995; Rothfuß et al., 1997; Rothfuß, 1997). Aktuelle Arbeiten befassen sich insbesondere mit der Erweiterung der flachheitsbasierten Methoden auf Systeme mit verteilten Parametern (SVPn), wobei speziell die Trajektorienplanung und der Steuerungsentwurf im Mittelpunkt der Untersuchungen stehen (Rudolph, 2003a). Hierbei werden bei parabolischen PDGLn Potenzreihenansätze verwendet, die die Parametrierung der Zustands- und Eingangsgrößen durch eine parametrierende Funktion (entsprechend dem flachen Ausgang) und deren Zeitableitungen bis zur unendlichen Ordnung ermöglichen siehe z.B. Fliess et al. (1997); Martin et al. (1997); Laroche et al. (1998); Fliess et al. (1998a,b); Laroche et al. (2000); Lynch and Rudolph (2002); Rudolph (2003a) und dortige Referenzen. In einer gewissen Analogie zum "early lumping", zeigt sich auch hier das Problem der Konvergenz, da durch eine geeignete Wahl der Trajektorien für die parametrierende Funktion, gleichmäßige Konvergenz des Reihenansatzes sichergestellt werden muss. Hieraus ergeben sich einige Einschränkungen:

- Gleichmäßige Konvergenz erzwingt die Wahl von Trajektorien aus gewissen GevreyKlassen, d.h. glatten Funktionen, deren Ableitungen bestimmten Wachstumseigenschaften unterliegen. Anderseits zeigen numerische Ergebnisse (Laroche et al., 2000), dass diese Einschränkung gelockert werden kann, was jedoch auf punktweise bzw. möglicherweise divergente Reihen führt, die geeignet summiert werden müssen.

- Der Konvergenznachweis liefert keine Information über die Konvergenzgeschwindigkeit der Reihe, die beispielsweise für konvektionsdominante Systeme wie die lineare DiffusionsKonvektions-Gleichung sehr klein sein kann (siehe Kapitel 2.1.2).

- Für nichtlineare DKRGn führt der Konvergenznachweis neben Bedingungen an die Trajektorien der parametrierenden Funktion zu weiteren Forderungen an die Systemparameter, die die Anwendbarkeit des Potenzreihenansatzes weiter einschränken (siehe Kapitel 2.1.3 und 2.2.2).

Diese Beobachtungen stellen den Ausgangspunkt dieser Arbeit dar, in der Lösungsansätze untersucht werden, um die genannten Einschränkungen von Potenzreihen zu überwinden. Au- 
ßerdem wird die weitreichende Anwendbarkeit von Potenzreihen zum Steuerungs- und Folgeregelungsentwurf für nichtlineare Diffusions-Konvektions-Reaktions-Systeme mit Randeingriffen erläutert. Speziell wird gezeigt, dass

- formale Potenzreihen ${ }^{1}$ in Verbindung mit geeigneten Methoden zur Konvergenzbeschleunigung und Summation divergenter Reihen ein mathematisches Gerüst zum Steuerungsentwurf für DKRGn mit Randeingriffen darstellen,

- formale Potenzreihen zum flachheitsbasierten Folgeregelungsentwurf mit Beobachter und Profilschätzung geeignet sind,

- der Steuerungs- und Regelungsentwurf basierend auf formalen Potenzreihen rechnergestützt mittels Computer-Algebra-Systemen durchgeführt werden kann,

- komplexe praktische Problemstellungen mittels des vorgeschlagenen Ansatzes behandelt werden können.

Zur Illustration der genannten Punkte wird der Einfachheit halber zunächst die lineare Wärmeleitungsgleichung behandelt. Mit diesen Ergebnissen wird die Erweiterung der Methodiken anhand des Modells eines nicht-isothermen Festbettreaktors bestehend aus zwei gekoppelten nichtlinearen DKRGn dargestellt.

\section{Steuerungsentwurf für DKRGn mit Randeingriff mittels Summationsmethoden}

Im Hinblick auf eine allgemeine Behandlung von parabolischen SVPn mit Randeingriff werden Potenzreihen in der Ortskoordinate mit zeitvariablen Koeffizienten angesetzt. Dies erlaubt für eine relativ große Klasse von DKRGn eine direkte Parametrierung von Systemzuständen und Eingängen in Abhängigkeit von einer Basisgröße, der sogenannten parametrierenden Funktion. Das prinzipielle Vorgehen wird im Folgenden zunächst anhand des Steuerungsentwurfs für die lineare Wärmeleitungsgleichung mit Randeingriff vorgestellt.

\section{Parametrierung der linearen Wärmeleitungsgleichung}

In der Modellgleichung des betrachteten Wärmeleiters werden im Weiteren alle Größen der Einfachheit halber als dimensionslos und normiert angenommen, d.h.

$$
\frac{\partial x(z, t)}{\partial t}=\lambda \frac{\partial^{2} x(z, t)}{\partial z^{2}}+\beta x(z, t), \quad z \in(0,1), t>0
$$

\footnotetext{
${ }^{1}$ Formal heißt in diesem Zusammenhang, dass der Konvergenzradius der Potenzreihe identisch Null sein kann.
} 
mit der Anfangsbedingung (AB)

$$
x(z, 0)=x_{0}(z), \quad z \in[0,1]
$$

und den konsistenten Randbedingungen (RBn)

$$
\begin{array}{rlrl}
\frac{\partial x}{\partial z}(0, t) & =0, & t>0 \\
p_{1} \frac{\partial x}{\partial z}(1, t)+r_{1} x(1, t) & =u(t), \quad t>0 .
\end{array}
$$

Die normierte Temperatur $x(z, t)$ kann über den Randeingriff $u(t)$ in (4) beeinflusst werden. Unter der formalen Annahme einer gleichmäßigen Konvergenz der Reihe

$$
\hat{x}(z, t)=\sum_{n=0}^{\infty} \hat{x}_{n}(t) z^{n}
$$

liefert die Substitution von (5) in PDGL (1) und RB (3) eine Differenzialrekursion 2. Ordnung für die zeitabhängigen Koeffizienten $\hat{x}_{n}(t), n \geq 2$ :

$$
\begin{aligned}
\hat{x}_{n+2}(t) & =\frac{\dot{\hat{x}}_{n}(t)-\beta \hat{x}_{n}(t)}{\lambda(n+2)(n+1)}, \quad n \in \mathbb{N}_{0} \\
\hat{x}_{1}(t) & =0 .
\end{aligned}
$$

Zur Lösung der Rekursion (6) ist neben (7) eine weitere Start-Bedingung notwendig. Diese kann beispielsweise aus dem Ansatz $x(0, t)=y(t)$ bestimmt werden, womit sich aus (5) ein weiterer Startwert ergibt:

$$
\hat{x}_{0}(t)=y(t)
$$

Somit ist die geschlossene Auswertung der Differenzialrekursion in Abhängigkeit von der eingeführten Größe $y(t)$ und deren Zeitableitungen möglich:

$$
\hat{x}(z, t)=\sum_{n=0}^{\infty} \frac{z^{2 n}}{\lambda^{n}(2 n) !} \sum_{i=0}^{n}\left(\begin{array}{c}
n \\
i
\end{array}\right)(-\beta)^{n-i} y^{(i)}(t) .
$$

Weiterhin kann aus dieser Parametrierung durch Differenziation mittels (4) die entsprechende Gleichung der Eingangsgröße angegeben werden:

$$
\hat{u}(t)=r_{1} \sum_{n=0}^{\infty} \frac{\sum_{i=0}^{n}\left(\begin{array}{c}
n \\
i
\end{array}\right)(-\beta)^{n-i} y^{(i)}(t)}{\lambda^{n}(2 n) !}+p_{1} \sum_{n=0}^{\infty} \frac{\sum_{i=0}^{n+1}\left(\begin{array}{c}
n+1 \\
i
\end{array}\right)(-\beta)^{n+1-i} y^{(i)}(t)}{\lambda^{n+1}(2 n+1) !}=: \sum_{n=0}^{\infty} \hat{u}_{n}(t) .
$$

Hieraus ist leicht ersichtlich, dass durch Vorgabe einer geeigneten $\mathcal{C}^{\infty}$-Funktion $y(t)$ die notwendige Steuerung $\hat{u}(t)$ bestimmt werden kann, die die Größe $x(0, t)$ entlang von $y(t)$ führt. Dies setzt die gleichmäßige Konvergenz der Reihe $\hat{x}(z, t)$ voraus. Hierzu kann leicht folgender Satz bewiesen werden (siehe auch (Widder, 1975, p.51) oder (Taylor, 1996, p.225)): 

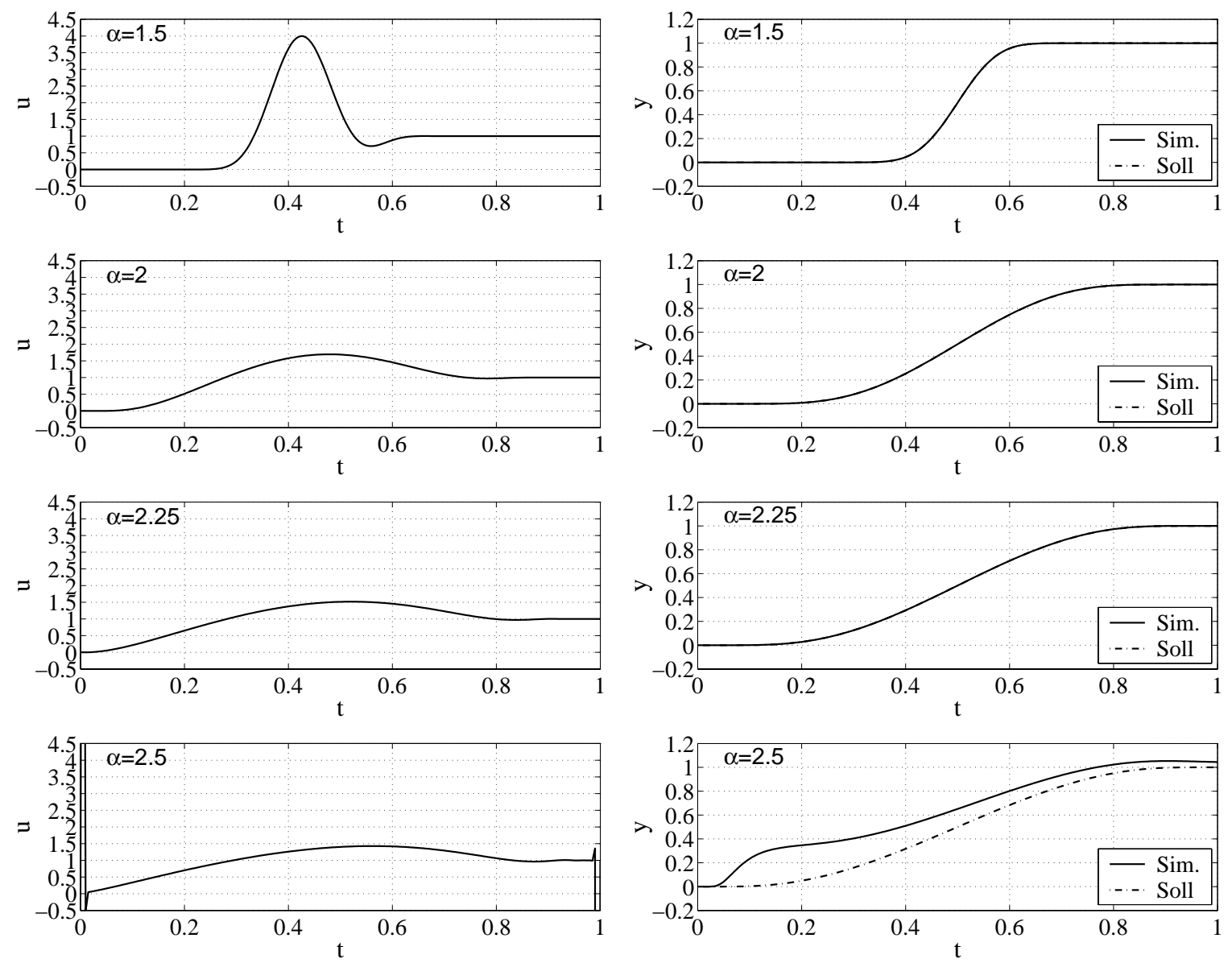

Bild 1: Vergleich der numerischen Ergebnisse für die Randsteuerung von (1)-(4) mit Parametern $\lambda=1$, $\beta=0, p_{1}=0$, und $r_{1}=1$ für die Soll-Trajektorie $y_{d}(t)=\Phi_{\gamma, T}(t)$ entsprechend Glg. (B.1) mit der Übergangszeit $T=1$ bei Variation von $\gamma$ bzw. $\alpha=1+1 / \gamma$. Links: Steuereingriff $u_{N}(t)$ aus (12) für $N=20$; rechts: Vergleich von Ist-Trajektorie $y(t)=x(0, t)$ und Soll-Trajektorie $y_{d}(t)$.

Satz 1. Die Reihe (9) konvergiert gleichmäßig für alle z gegen die Lösung von (1) mit den RBn (3), (4), falls y(t) eine Gevrey-Funktion der Ordnung $\alpha<2$ ist, d.h.

$$
\sup _{t \in \mathbb{R}^{+}}\left|y^{(n)}(t)\right| \leq \frac{M}{R^{n}}(n !)^{\alpha}, \quad n \in \mathbb{N}_{0}, M, R \in \mathbb{R}^{+} .
$$

Offensichtlich bedingt die Realisierung der Steuerfunktion den Abbruch der Reihe (10) an einer geeigneten Summationsgrenze $N \in \mathbb{N}$, so dass anstelle von $\hat{u}(t)$ die Steuerung durch

$$
u_{N}(t)=\sum_{n=0}^{N} \hat{u}_{n}(t)
$$

approximiert wird. Abbildung 1 zeigt Simulationsergebnisse für die Anwendung der Steuerfunktion (12) auf ein semi-diskretisiertes Modell des Wärmeleiters bei Variation der GevreyOrdnung $\alpha$. Dabei wird die in Anhang B vorgestellte Funktion $\Phi_{\gamma, T}(t)$ (B.1) der GevreyOrdnung $\alpha=1+\frac{1}{\gamma}$ als Solltrajektorie für $y(t)$ vorgegeben. Diese Funktion mit kompaktem 
Träger erlaubt insbesondere die Realisierung von Übergängen innerhalb endlicher Zeitintervalle $t \in[0, T]$. Wie in Kapitel 4.1 gezeigt wird, kann durch die Vorgabe von $y(t)$ direkt ein Übergang zwischen entsprechenden stationären Profilen erzeugt werden.

Erwartungsgemäß treten für $\alpha \leq 2$ keine Abweichungen zwischen Soll- und Ist-Trajektorie auf, wobei mit ansteigendem $\alpha$ eine Abnahme der Steueramplitude zu beobachten ist. Anderseits zeigen sich an den Rändern des Ortsbereichs störende Oszillationen bei Überschreiten des Schwellenwertes von $\alpha=2$, deren Intensität mit zunehmendem $\alpha$ ansteigt. Für $\alpha \approx 2$ ist ein glatter Steuereingang mit gutem Folgeverhalten zu beobachten, obwohl theoretisch divergentes Verhalten auftreten sollte. Dies ist in diesem Fall durch die Wahl von $N=20$ begründet, wobei die Addition weiterer Reihenglieder $\hat{u}_{n}(t), n>N$, zu einem Verhalten ähnlich dem für $\alpha=2.5$ führt. Dieses für einige divergente Reihen typische Verhalten (Knopp, 1964) wird beispielsweise in Laroche et al. (2000) in der Anwendung der sogenannten "Summation zum kleinsten Term" angewandt, bei der Reihenglieder solange addiert werden, bis der betragsmäßig kleinste Term erreicht wird. Die Anwendbarkeit dieser eher heuristischen Methode ${ }^{2}$ ist jedoch deutlich eingeschränkt (Wagner et al., 2004). Andererseits existieren weitaus geeignetere Summationsmethoden, die Gegenstand der weiteren Betrachtungen sind.

\section{Der Begriff der formalen Potenzreihenparametrierung}

Ausgehend von den Ergebnissen für den linearen Wärmeleiter kann der Potenzreihenansatz direkt auf komplexere PDGLn mit Randeingriff erweitert werden. Im Rahmen dieser Arbeit werden speziell die lineare Diffusions-Konvektions-Gleichung, skalare DKRGn mit polynomialen Nichtlinearitäten sowie Systeme nichtlinearer parabolischer PDGLn untersucht — siehe Kapitel 2 und das Anwendungsbeispiel am Ende dieser Kurzfassung.

Wie einleitend erwähnt, stellen der Konvergenznachweis bzw. die sich daraus ergebenden Bedingungen deutliche Einschränkungen der Anwendbarkeit des Potenzreihenansatzes dar. Hieraus ergibt sich die Motivation, die Betrachtungen mit sogenannten formalen Potenzreihen fortzusetzen, deren Konvergenzradius ohne Einschränkung gleich Null sein kann (Balser, 2000). Somit sind die Rechenoperationen im Folgenden als rein formal anzusehen, da sie typischerweise gleichmäßige Konvergenz der Reihe voraussetzen. Hieraus kann eine wesentliche Verallgemeinerung der Parametrierbarkeit von DKRGn erreicht werden, die zur Definition der sogenannten formalen Potenzreihenparametrierung (FPRP) führt (Meurer and Zeitz, 2004a; Wagner et al., 2004; Meurer and Zeitz, 2005).

Definition 1 (Formale Potenzreihenparametrierung). Eine K-dimensionale Menge von nichtlinearen DKRGn 2. Ordnung mit Randeingriffen

$$
G_{i}\left(\frac{\partial \boldsymbol{x}(z, t)}{\partial t}, \boldsymbol{x}(z, t), \frac{\partial \boldsymbol{x}(z, t)}{\partial z}, \frac{\partial^{2} \boldsymbol{x}(z, t)}{\partial z^{2}}\right)=0, \quad z \in(0,1), \quad t>0, \quad i=1, \ldots, K
$$

\footnotetext{
${ }^{2}$ Es ist beispielsweise im Allgemeinen nicht möglich, den kleinsten Term a priori zu bestimmen.
} 


$$
\begin{aligned}
& R_{0, j}\left(\boldsymbol{x}(0, t), \frac{\partial \boldsymbol{x}}{\partial z}(0, t), \boldsymbol{u}_{0}(t)\right)=0, t>0, \quad j=1, \ldots, K_{0} \\
& R_{1, l}\left(\boldsymbol{x}(1, t), \frac{\partial \boldsymbol{x}}{\partial z}(1, t), \boldsymbol{u}_{1}(t)\right)=0, \quad t>0, \quad l=1, \ldots, K_{1} \quad\left(K_{0}+K_{1}=2 K\right) \\
& \boldsymbol{x}(z, 0)=\boldsymbol{x}_{0}(z), \quad z \in[0,1]
\end{aligned}
$$

definiert auf $(z, t) \in[0,1] \times \mathbb{R}^{+}$mit dem Zustandsvektor $\boldsymbol{x}(z, t)=\left[x_{1}(z, t), \ldots, x_{K}(z, t)\right]^{T}$ und den Eingangsgrößen $\boldsymbol{u}(t)$ aus geeigneten Funktionenräumen $\mathcal{X}[0,1]$ bzw. $\mathcal{U}$, wird formal potenzreihenparametrierbar (FPRP) genannt, falls eine formale Potenzreihe

$$
\hat{\boldsymbol{x}}(z, t)=\sum_{n=0}^{\infty} \hat{\boldsymbol{x}}_{n}(t) p_{n}(z)
$$

mit $p_{n}(z)$ einem geeigneten Polynom in z der Ordnung $n$ existiert, die formal (13)-(16) erfüllt und deren Koeffizienten $\hat{\boldsymbol{x}}_{n}(t)$ durch eine parametrierende Funktion

$$
\boldsymbol{y}(t)=\boldsymbol{H}\left(\boldsymbol{x}(0, t), \frac{\partial \boldsymbol{x}}{\partial z}(0, t), \boldsymbol{x}(1, t), \frac{\partial \boldsymbol{x}}{\partial z}(1, t), \boldsymbol{u}_{0,1}(t)\right)
$$

mit $\operatorname{dim} \boldsymbol{y}=\operatorname{dim} \boldsymbol{u}_{0}+\operatorname{dim} \boldsymbol{u}_{1}$ und ihren Zeitableitungen ausgedrückt werden können, d.h.

$$
\hat{\boldsymbol{x}}_{n}(t)=\boldsymbol{\Upsilon}_{n}\left(\boldsymbol{y}(t), \dot{\boldsymbol{y}}(t), \ldots, \boldsymbol{y}^{\left(r_{x}\right)}(t)\right)
$$

mit $r_{x} \in \mathbb{N}_{0}$, wobei ggf. $r_{x} \rightarrow \infty$ mit $n \rightarrow \infty$. Die Eingangsgrößen $\boldsymbol{u}_{0,1}(t)$ werden FPRP genannt, falls (13)-(16) FPRP sind und (14), (15) nach $\boldsymbol{u}_{0,1}(t)$ (explizit) auflösbar sind.

Diese Definition drückt im Wesentlichen die Parametrierbarkeit der Zustands- und Eingangsgrößen eines Systems von DKRGn aus, womit sich eine gewisse Ähnlichkeit zum Flachheitsbegriff ergibt. Insbesondere kann FPRP als eine Art konstruktiver Steuerbarkeitsnachweis interpretiert werden, da direkt durch Vorgabe einer Trajektorie für die parametrierende Größe $\boldsymbol{y}(t)$ formal die zugehörige Steuerung $\boldsymbol{u}(t)$ ermittelt werden kann, die das System im offenen Kreis entlang dieser Trajektorie führt. Speziell für lineare DKRGn kann im Fall gleichmäßiger Reihenkonvergenz gezeigt werden, dass FPRP approximative Steuerbarkeit impliziert (Laroche, 2000, Prop. 2.6.2).

Diese verallgemeinerte formale Betrachtungsweise bedingt, dass aus der parametrierten und möglicherweise divergenten formalen Potenzreihe (17), (19) mittels geeigneter mathematischer Methoden ein sinnvoller Grenzwert extrahiert werden muss. Im Weiteren wird gezeigt, dass dies über geeignete Summationsmethoden erreicht werden kann.

\section{Steuerungsentwurf mittels Summationsmethoden}

Die gebräuchlichste Summationsmethode, obwohl nicht als solche bezeichnet, stellt die Grenzwertbildung in der klassischen Partialsummenbildung dar, d.h.

$$
\mathcal{S}_{P}\left(\sum_{n=0}^{\infty} \hat{x}_{n} z^{n}\right)=\lim _{N \rightarrow \infty} \sum_{n=0}^{N} \hat{x}_{n} z^{n} .
$$


Hierbei kann $\mathcal{S}_{P}$ als lineares Funktional aufgefasst werden, das jede konvergente Potenzreihe auf ihre natürliche Summe abbildet. Offensichtlich handelt es sich hierbei um eine schwache Methode, da sie nur auf konvergente Reihen anwendbar ist, sie zeigt jedoch den prinzipiellen Ansatz. Im Folgenden sei $\mathcal{S}_{V}$ ein lineares Funktional auf einem linearen Raum $X_{\mathcal{S}}$ von Folgen bzw. Reihen und $\left\{a_{n}(\xi)\right\}_{n \in \mathbb{N}_{0}}$ eine Folge von Funktionen in $\xi \geq 0$. Des Weiteren soll die Wirkung von $\mathcal{S}_{V}$ auf eine formale Reihe $\hat{x}(z)=\sum_{n=0}^{\infty} \hat{x}_{n} z^{n}$ mit $z \in \mathbb{C}$ folgendermaßen gegeben sein:

$$
\mathcal{S}_{V}\left(\sum_{n=0}^{\infty} \hat{x}_{n} z^{n}\right)=\lim _{\xi \rightarrow \infty} \sum_{n=0}^{\infty} a_{n}(\xi) \hat{x}_{n} z^{n}=\lim _{\xi \rightarrow \infty} \hat{x}^{A}(z ; \xi) .
$$

Falls $\hat{x}^{A}(z ; \xi)$ für $|z|<\rho$ konvergiert, so definiert dies eine Familie von holomorphen Funktionen in einem Kreis um $z=0$ mit Radius $\rho$. Konvergiert der Grenzwert für $\xi \rightarrow \infty$ gleichmäßig in $z$, so definiert $x(z)=\lim _{\xi \rightarrow \infty} x^{A}(z ; \xi)$ eine holomorphe Funktion in einem Gebiet $\Omega$ der komplexen Ebene (Ramis, 1993). In diesem Fall wird die Reihe $\hat{x}(z)$ als A-summierbar im Gebiet $\Omega$ bezeichnet, und die Funktion $x(z)=\left(\mathcal{S}_{V} \hat{x}\right)(z)$ kann als die $A$-Summe von $\hat{x}(z)$ aufgefasst werden. Der Raum $X_{\mathcal{S}}$ aller $A$-summierbaren Reihen wird als Summierbarkeitsbereich der Summationsmethode $\mathcal{S}_{V}$ bezeichnet. Eine allgemeine Diskussion dieser Ergebnisse ist beispielsweise in Hardy (1949) zu finden.

Um eine Summationsmethode zur Lösung von Differenzialgleichungen einzusetzen, muss diese weitere Bedingungen erfüllen (Ramis, 1993; Balser, 2000). Hierzu muss (i) der Summierbarkeitsbereich $X_{\mathcal{S}}$ die Struktur einer Differenzialalgebra aufweisen und eine Erweiterung des Raums der konvergenten Reihen darstellen; (ii) $\mathcal{S}_{V}$ regulär sein, d.h. jede konvergente Reihe auf ihre natürliche Summe aufsummieren; (iii) $\mathcal{S}_{V}$ einen linearen Homomorphismus darstellen, der Produkte auf Produkte sowie Ableitungen auf Ableitungen abbildet; (iv) $\mathcal{S}_{V}$ der formalen eine gegebene Differenzialgleichung (DGL) erfüllenden - Reihe $\hat{x}(z)$ eindeutig eine Summe $x(z)=\left(\mathcal{S}_{V} \hat{x}\right)(z)$ zuweisen, die ebenfalls die DGL erfüllt. Zur Behandlung von PDGLn müssen noch weitere Bedingungen formuliert werden, wie insbesondere die gleichmäßige Summierbarkeit, um Ableitungen der variablen Reihenkoeffizienten nach der Zeit $t$ zuzulassen (vgl. Glg. $(6))$.

Eine Summationsmethode, welche die genannten Forderungen erfüllt, ist die sogenannte $k^{-}$ Summation, die von Ramis (1980) als eine Erweiterung der Arbeit von Borel (1928) zur Summierbarkeit von divergenten Reihen eingeführt wird. Im Folgenden wird nur eine der möglichen äquivalenten Definitionen herangezogen (Balser and Braun, 2000):

$$
x(z) \cong \hat{x}(z)=\lim _{\xi \rightarrow \infty} \frac{\sum_{n=0}^{\infty} s_{n}(z) \frac{\xi^{n}}{\Gamma\left(1+\frac{n}{k}\right)}}{\sum_{n=0}^{\infty} \frac{\xi^{n}}{\Gamma\left(1+\frac{n}{k}\right)}}=:\left(\mathcal{S}_{B}^{k} \hat{x}\right)(z),
$$

wobei $s_{n}(z)$ die $n$-te Partialsumme darstellt. Für eine ausführliche Diskussion der Eigenschaften sowie des Nachweises der $k$-Summierbarkeit wird auf Ramis (1980); Malgrange (1995); Balser $(1994,2000)$ und deren Referenzen verwiesen. Beispielsweise erlaubt die Anwendung von $\mathcal{S}_{B}^{k}$ die Summation der geometrischen Reihe $\sum_{n=0}^{\infty} z^{n}$ außerhalb ihres Konvergenzbereiches $|z|<1$, d.h. in der gesamten komplexen Halbebene $\Re z<1$, zur Summe $\frac{1}{1-z}$. 
Aktuelle Arbeiten betreffen insbesondere den Nachweis der $k$-Summierbarkeit von formalen Potenzreihenlösungen für lineare PDGLn, wobei fast ausschließlich das Cauchy-Problem auf einem unendlich ausgedehnten Ortsbereich betrachtet wird (Lutz et al., 1996; Balser, 1999; Balser and Miyake, 1999; Hibino, 2001, 2003; Balser, 2003; Balser and Malek, 2003; Balser and Kostov, 2003). Diese Ergebnisse sind somit nicht zur Behandlung der formalen Lösung von den betrachteten Randsteuerungsproblemen für DKRGn anwendbar. Des Weiteren können für nichtlineare Probleme nur eine endliche Anzahl von Reihenkoeffizienten bestimmt werden, so dass die beiden Grenzwertprozesse in der Definition (20) der $k$-Summation $(n \rightarrow \infty$ und $\xi \rightarrow \infty)$ nicht ausgewertet werden können. Dies ist der Grund für die Definition der sogenannten $(N, \xi)$-approximativen $k$-Summe (Wagner, 2003), (Wagner et al., 2004; Meurer and Zeitz, 2004a)

Definition 2. Die $(N, \xi)$-approximative $k$-Summe $\mathcal{S}_{k}^{N, \xi}$ einer $k$-summierbaren formalen Potenzreihe $\hat{x}(z)=\sum_{n=0}^{\infty} \hat{x}_{n} z^{n}$ wird als

$$
\mathcal{S}_{k}^{N, \xi} \hat{x}(z)=\frac{\sum_{n=0}^{N} s_{n}(z) \frac{\xi^{n}}{\Gamma\left(1+\frac{n}{k}\right)}}{\sum_{n=0}^{N} \frac{\xi^{n}}{\Gamma\left(1+\frac{n}{k}\right)}}
$$

mit der $n$-ten Partialsumme $s_{n}(z)=\sum_{j=0}^{n} \hat{x}_{j} z^{j}$ definiert.

Wie anhand einiger klassischer Beispiele gezeigt werden kann (siehe Kapitel 3 dieser Arbeit), vereint diese Summationsvorschrift in guter Näherung die günstigen Eigenschaften der $k^{-}$ Summation mit den Anforderungen, die sich aus der Implementierung der Steuerung ergeben.

Im Folgenden wird die Anwendung der $(N, \xi)$-approximativen $k$-Summe am Beispiel des Randsteuerungsentwurfs für die lineare Wärmeleitungsgleichung (1)-(4) demonstriert. Hierzu wird die Partialsummenbildung (12) zur Bestimmung der Steuerung durch $\mathcal{S}_{k}^{N, \xi}$ ersetzt, so dass sich die Steuerung mit Koeffizienten $\hat{u}_{n}(t)$ entsprechend (10) zu

$$
u_{k}^{N, \xi}(t)=\mathcal{S}_{k}^{N, \xi}\left(\sum_{n=0}^{\infty} \hat{u}_{n}(t)\right)
$$

ergibt. Abbildung 2 zeigt die numerischen Ergebnisse für die Anwendung von $u_{k}^{N, \xi}(t)$ auf ein semi-diskretisiertes Modell des linearen Wärmeleiters (1)-(4). Die Solltrajektorien sind hierbei so gewählt, dass durch Erhöhung der Gevrey-Ordnung (11) über den Schwellenwert von $\alpha=2$ ein möglichst glatter Übergang zwischen den stationären Werten mit minimaler Steueramplitude erreicht werden kann. Die Anwendung der $(N, \xi)$-approximativen $k$-Summe (22) ermöglicht offensichtlich die Summation der divergenten Reihenlösung zu einer sinnvollen Steuerfunktion, die das vorgegebene Folgeverhalten hinreichend genau realisiert. Wie in Abbildung 1 gezeigt, kann dies nicht mit der klassischen Partialsummenbildung erreicht werden. Neben den sich ergebenden erweiterten Möglichkeiten der Trajektorienplanung können geeignete Summationsmethoden auch zur Konvergenzbeschleunigung langsam konvergierender Reihen herangezogen werden, wie z.B. im Fall formaler Potenzreihenlösungen der linearen Diffusions-KonvektionsGleichung (siehe Kapitel 4.3.2). Des weiteren bietet, wie Vergleiche mit sogenannten verallgemeinerten Summationsprozessen (Weniger, 1994) zeigen, speziell die $(N, \xi)$-approximative 

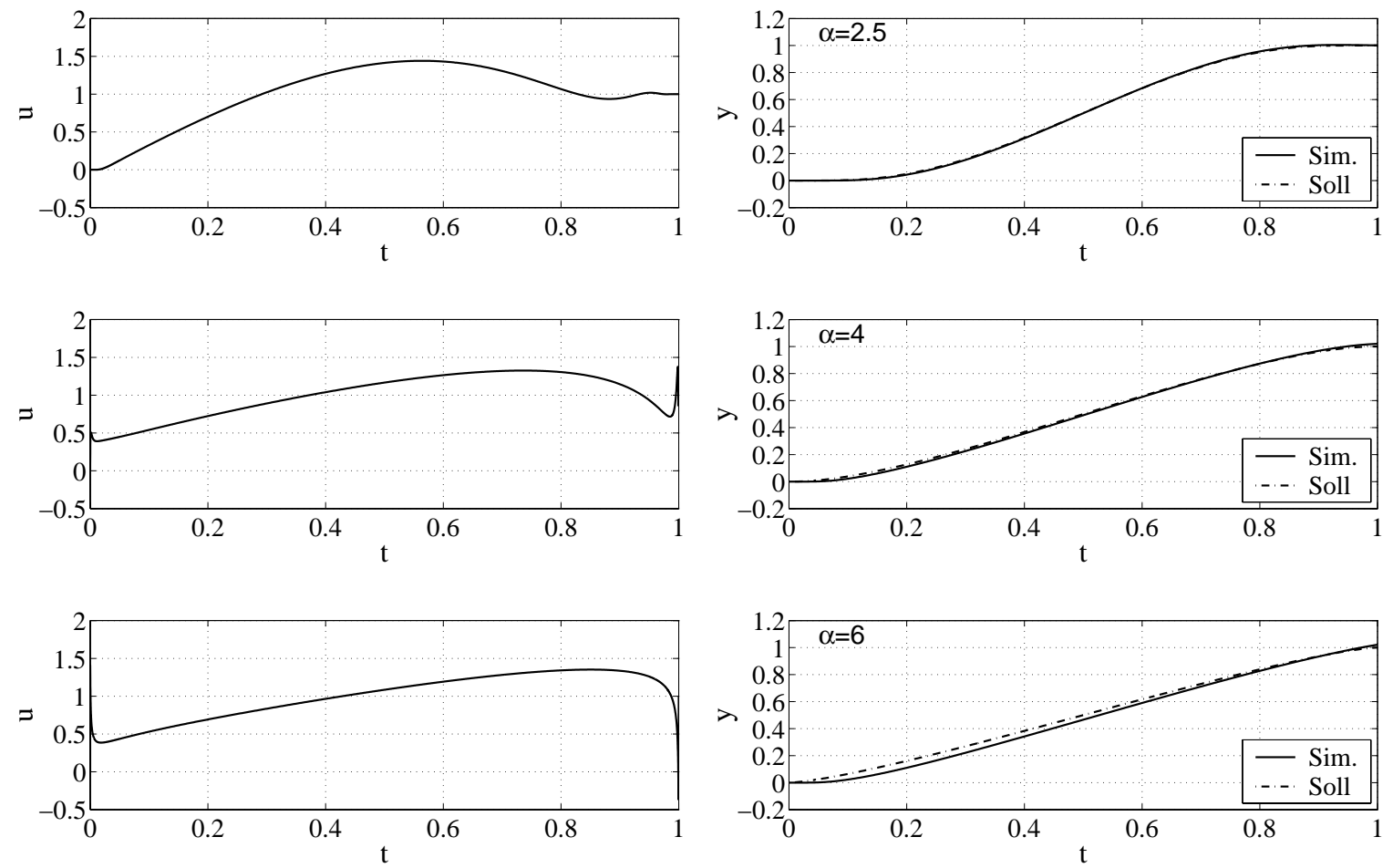

Bild 2: Numerische Simulation des linearen Wärmeleiters (1)-(4) mit Randsteuerung aus $(N, \xi)-$ approximativer $k$-Summe (21) für Parameter $\lambda=1, \beta=0, p_{1}=0$ und $r_{1}=1$ und Soll-Trajektorie $y_{d}(t)=\Phi_{\gamma, T}(t)$ entsprechend Glg. (B.1) mit einer Übergangszeit $T=1$ bei Variation der GevreyOrdnung $1+1 / \gamma=\alpha \in\{2.5,4,6\}$. Links: Steuereingriff $u_{N}(t)$ aus (12) für $N=20$; rechts: Vergleich von Ist-Trajektorie $y(t)=x(0, t)$ und Soll-Trajektorie $y_{d}(t)$.

k-Summe weitreichende Möglichkeiten zum Steuerungsentwurf für Systeme nichtlinearer parabolischer PDGLn mit Randeingriff, wie dies anhand des Anwendungsbeispiels am Ende dieser Kurzfassung gezeigt wird. Ein allgemeiner Zugang wird in Kapitel 4.4 vorgestellt.

Im Allgemeinen ist die Anwendbarkeit einer reinen Steuerung stark eingeschränkt, so dass der Regelkreis durch eine stabilisierende Rückführung zur Kompensation von Modellfehler und exogenen Störungen robust gemacht werden muss. Im Folgenden wird gezeigt, dass hierzu ebenfalls formale Potenzreihen herangezogen werden können.

\section{Flachheitsbasierter Folgeregelungsentwurf für DKRGn mit Randeingriffen}

Ausgehend von obigen Ergebnissen zum Randsteuerungsentwurf ermöglicht eine NeuInterpretation der formalen Potenzreihenparametrierung die Herleitung eines endlich-dimensionalen und differenziell flachen Entwurfsmodells in den Koeffizienten der formalen Potenzreihe (Meurer, 2002), (Meurer and Zeitz, 2003b). Dies erlaubt die direkte Übertragung 
der bekannten flachheitsbasierten Entwurfsmethoden zur Folgeregelung mit Beobachter auf DKRGn.

Der Einfachheit halber wird wieder das Beispiel des linearen Wärmeleiters mit Randeingriff (1)-(4) betrachtet (für eine ausführliche Betrachtung sei auf Kapitel 5.1.1 verweisen). Die Idee der folgenden Modellreduktion basiert auf der Interpretation der durch Einsetzen des Potenzreihenansatzes ermittelten Differenzialrekursion (6) als ein System gewöhnlicher DGLn in den Reihenkoeffizienten $\hat{x}_{2 n}(t) .{ }^{3}$ Unter der formalen Annahme der gleichmäßigen Konvergenz ist es ausreichend, nur die ersten $N \in \mathbb{N}$ Koeffizienten zu betrachten, d.h.

$$
\begin{aligned}
\frac{d \hat{x}_{2 n}}{d t} & =(2 n+2)(2 n+1) \lambda \hat{x}_{2 n+2}+\beta \hat{x}_{2 n}, \quad n=0,1, \ldots, N-2 \\
\frac{d \hat{x}_{2 N-2}}{d t} & =(2 N)(2 N-1) \lambda \hat{x}_{2 N}+\beta \hat{x}_{2 N-2} .
\end{aligned}
$$

Der Koeffizient $\hat{x}_{2 N}(t)$ in der letzten DGL kann durch Auswertung der inhomogenen Randbedingung (4) mittels der Potenzreihe $\hat{x}(z, t)$ und deren Abbruch nach $2 N-1$ Gliedern in Abhängigkeit von $\hat{x}_{2 n}(t), n=0,1, \ldots, N-1$, und Eingang $\hat{u}(t)$ ausgedrückt werden. Alternativ kann anstelle der Partialsummenbildung die $(N, \xi)$-approximate $k$-Summe der Reihe für $\hat{u}(t)$ verwendet werden, was speziell die restriktive Annahme der gleichmäßigen Konvergenz umgeht. Dies führt auf ein lineares System gewöhnlicher DGLn

$$
\begin{aligned}
\dot{\hat{\boldsymbol{x}}}^{\prime}{ }_{2 N-2} & =A \hat{\boldsymbol{x}}_{2 N-2}^{\prime}+\boldsymbol{b} \hat{u}, \quad t>0, \quad \hat{\boldsymbol{x}}_{2 N-2}^{\prime}(0)=\hat{\boldsymbol{x}}_{2 N-2,0}^{\prime} \in \mathbb{R}^{N} \\
y & =\boldsymbol{c}^{T} \hat{\boldsymbol{x}}_{2 N-2}^{\prime} .
\end{aligned}
$$

mit dem Zustand $\hat{\boldsymbol{x}}_{2 N-2}^{\prime}=\left[x_{0}, x_{2}, \ldots, x_{2 N-2}\right]^{T}$ in Abhängigkeit von dem Eingang $\hat{u}$. Wie in Kapitel 5.1.1 für den Wärmeleiter ausführlich gezeigt, liefert dieses endlich-dimensionale Modell eine hinreichend genaue Approximation der dominanten Dynamik des Originalsystems (1)(4). Die Systemmatrix $A$ besitzt eine charakteristische Bandstruktur, weshalb (25) bzgl. der parametrierenden Funktion (26) in einer verallgemeinerten Regelungs-Normalform vorliegt. Somit kann leicht, z.B. mittels klassischer Eigenwertvorgabe, eine asymptotisch stabilisierende Folgeregelung entworfen werden:

$$
b_{\xi, k}^{N} \hat{u}=-\underbrace{\boldsymbol{h}^{T} \hat{\boldsymbol{x}}_{2 N-2}^{\prime}}_{\text {Kompensation }}-\underbrace{\boldsymbol{s}^{T} \hat{\boldsymbol{x}}_{2 N-2}^{\prime}}_{\text {Stabilisierung }}-\underbrace{\frac{p_{0} \int_{0}^{t}\left(y(\tau)-y_{d}(\tau)\right) d \tau}{(2 N-2) ! \lambda^{N-1}}}_{\text {Integral-Anteil }}+\underbrace{\frac{y_{d}^{(N)}(t)+\sum_{n=0}^{N-1} p_{n} y_{d}^{(n)}(t)}{(2 N-2) ! \lambda^{N-1}}}_{\text {Steuerung }} .
$$

Diese gliedert sich in den zustandsrückführenden Teil, bestehend aus Kompensation und Stabilisierung, einen Integral-Anteil zur Kompensation statischer Abweichungen sowie einen Steuerungsanteil zur Aufprägung des Sollverlaufs. Untersuchungen zur Stabilität sowie numerische Simulationsergebnisse für den linearen Wärmeleiter sind in Kapitel 5.1.1 zu finden.

Insbesondere ist hierbei zu betonen, dass der Ansatz zur Herleitung eines endlich-dimensionalen Entwurfsmodells auf alle nichtlinearen Diffusions-Konvektions-Reaktions-Systeme mit Randeingiff anwendbar ist, welche die Bedingungen von Definition 1 erfüllen. Wie in Kapitel 5 detailliert gezeigt, führt diese Art der Modellreduktion im Allgemeinen auf ein endlich-dimensionales

\footnotetext{
${ }^{3}$ Aufgrund von $\hat{x}_{1}(t)=0$ aus Glg. (7) kann leicht gezeigt werden, dass $\hat{x}_{2 n+1}(t)=0, \forall n \in \mathbb{N}_{0}$.
} 
nichtlineares inhärent flaches Mehrgrößensystem. Dies erlaubt eine direkte Übertragung der bekannten flachheitsbasierten Methodik zum Folgeregelungsentwurf auf nichtlineare DKRGn.

Im Folgenden wird die weitreichende Anwendbarkeit der in dieser Arbeit vorgeschlagenen Kombination von formalen Potenzreihen und geeigneten Summationsmethoden zum Steuerungsund Folgeregelungsentwurf für nichtlineare DKRGn mit Randeingriff anhand des Modells eines nicht-isothermen Rohrreaktors erläutert.

\section{Anwendungsbeispiel: Modell eines nicht-isothermen Rohrreaktors mit Randeingriff}

Hierzu wird das pseudo-homogene Dispersionsmodells eines Rohrreaktors im Fall einer irreversiblen Flüssigphasen-Reaktion $A \rightarrow B$ betrachtet. Die Modellgleichungen des Rohrreaktors in dimensionsloser Form ergeben sich für $z \in(0,1), t>0$ entsprechend (Ray, 1981; Jensen and Ray, 1982) zu

$$
\frac{\partial \boldsymbol{x}}{\partial t}=\left(\begin{array}{cc}
\frac{L e}{P e_{1}} & 0 \\
0 & \frac{1}{P e_{2}}
\end{array}\right) \frac{\partial^{2} \boldsymbol{x}}{\partial z^{2}}+\left[\begin{array}{c}
L e \frac{\partial x_{1}}{\partial z}+\operatorname{DaLe}\left(1-x_{1}\right) \exp \left(\frac{x_{2}}{1+x_{2} / \sigma}\right) \\
\frac{\partial x_{2}}{\partial z}-\beta\left(x_{2}-x_{2 w}\right)+B D a\left(1-x_{1}\right) \exp \left(\frac{x_{2}}{1+x_{2} / \sigma}\right)
\end{array}\right]
$$

mit dem Zuständsvektor $\boldsymbol{x}(z, t):=\left[x_{1}(z, t), x_{2}(z, t)\right]^{T}$, den Danckwerts Randbedingungen

$$
\begin{array}{ll}
\frac{\partial \boldsymbol{x}}{\partial z}(0, t)=\mathbf{0}, & t>0 \\
\left(\begin{array}{cc}
\frac{1}{P e_{1}} & 0 \\
0 & \frac{1}{P e_{2}}
\end{array}\right) \frac{\partial \boldsymbol{x}}{\partial z}(1, t)+\boldsymbol{x}(1, t)=\boldsymbol{u}(t), & t>0
\end{array}
$$

und den Anfangsbedingungen

$$
\boldsymbol{x}(z, 0)=\boldsymbol{x}_{0}(z), \quad z \in[0,1] .
$$

Eine genaue Beschreibung der dimensionslosen Kennzahlen ist in Kapitel 2.2.2 zu finden, wobei speziell die Peclet-Zahlen $P e_{1}$ und $P e_{2}$ der Massen- bzw. Energiebilanz, die das Verhältnis von Konvektion zu Diffusion beschreiben, für die weiteren Betrachtungen hervorzuheben sind. Die weiteren Kennzahlen sind die Lewis-Zahl Le, die die Packungsdichte beschreibt (z.B. gilt nach Jensen and Ray (1982) Le $\approx 500$ für den gepackten Festbettreaktor) sowie die Damköhler-Zahl $D a$, welche die Reaktion kennzeichnet. Die dimensionslosen Zustandsgrößen $x_{1}(z, t)$ und $x_{2}(z, t)$ können im Wesentlichen als Umsatz und normierte Temperatur interpretiert werden.

Unter der Annahme, dass die Exponentialfunktion in (27) durch Polynome im Zustand $x_{2}$ approximiert werden kann, liefert das formale Einsetzen der Potenzreihe

$$
\hat{\boldsymbol{x}}(z, t)=\sum_{n=0}^{\infty} \hat{\boldsymbol{x}}_{n}(t) z^{n}
$$



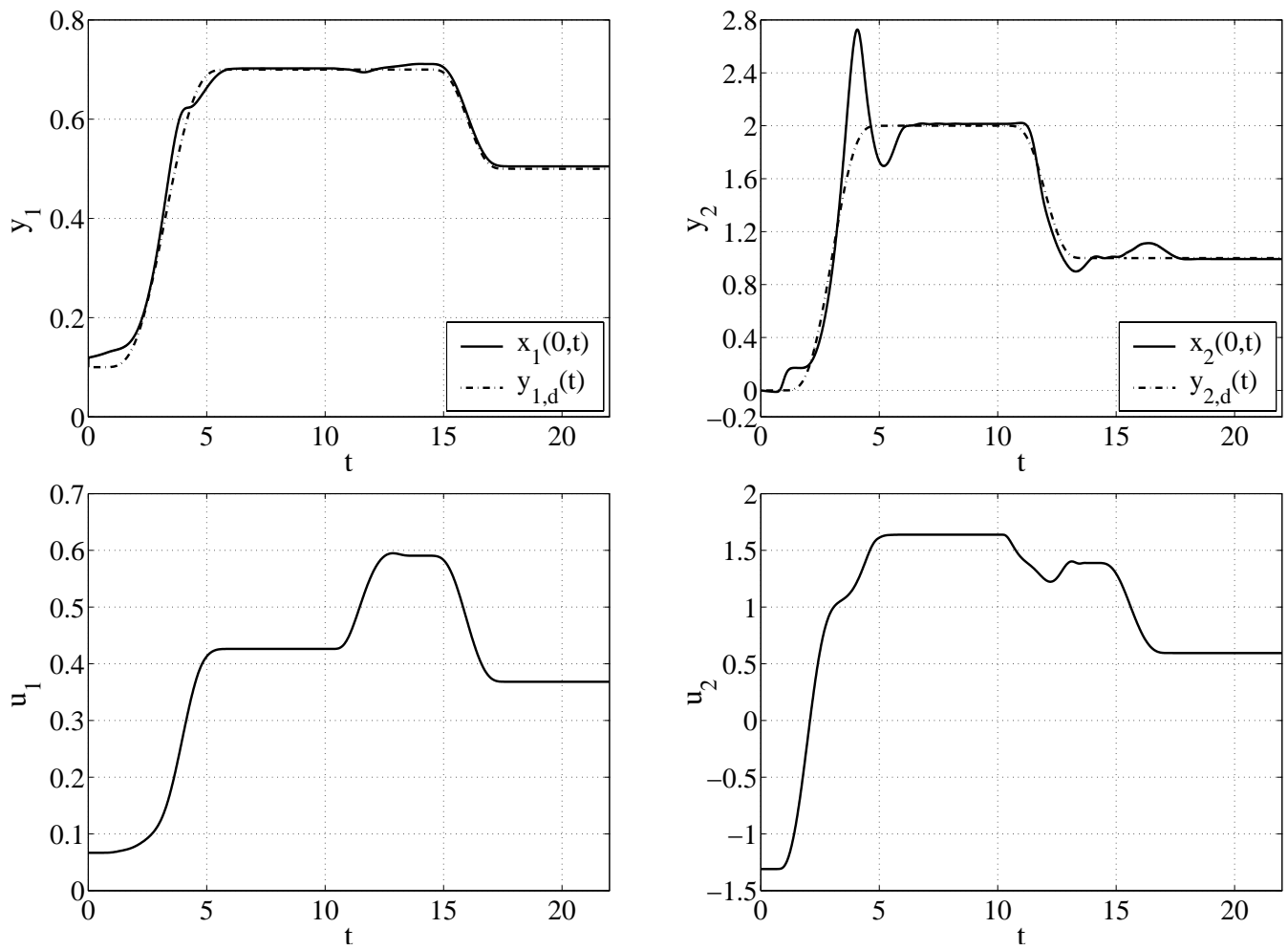

Bild 3: Numerische Simulation des Rohrreaktormodells (27)-(30) mit Randsteuerung (34) für $P e_{1}=$ 200, $P e_{2}=100, L e=500$ und weiteren Parametern entsprechend Anhang D.2. Oben: Folgeverhalten für $x_{1}(0, t)$ und $x_{2}(0, t)$; Unten: zeitlicher Verlauf der Eingriffsgrößen (34).

in die PDGLn (27) unter Auswertung von Produkten und Potenzen der Reihen $\hat{x}_{1}(z, t)$ und $\hat{x}_{2}(z, t)$ mittels der Cauchyschen Produktformel ein gekoppeltes System von Differenzialrekursionen zweiter Ordnung für die Reihenkoeffizienten $\hat{\boldsymbol{x}}_{n}(t), n \geq 2$. Die notwendigen vier Startbedingungen zur rekursiven Auswertung dieser Gleichungen können aus den homogenen RBn (28) sowie der Einführung einer parametrierenden Funktion $\boldsymbol{y}(t)=\boldsymbol{x}(0, t)$ zu $\hat{\boldsymbol{x}}_{0}(t)=\boldsymbol{y}(t)$ und $\hat{\boldsymbol{x}}_{1}(t)=0$ bestimmt werden ${ }^{4}$. Es zeigt sich, dass der Zustand $\boldsymbol{x}(z, t)$ und Randeingriff $\boldsymbol{u}(t)$ Parametrierungen der Form

$$
\begin{aligned}
\hat{\boldsymbol{x}}(z, t)= & \sum_{n=0}^{\infty}\left[\hat{\boldsymbol{x}}_{2 n}\left(\boldsymbol{y}(t), \dot{\boldsymbol{y}}(t), \ldots, \boldsymbol{y}^{(n)}(t)\right) z^{2 n}+\hat{\boldsymbol{x}}_{2 n+1}\left(\boldsymbol{y}(t), \dot{\boldsymbol{y}}(t), \ldots, \boldsymbol{y}^{(n)}(t)\right) z^{2 n+1}\right] \\
\hat{u}_{i}(t) & =y_{i}(t)+\sum_{n=0}^{\infty}\left[1+\frac{2 n+1}{P e_{i}}\right] \hat{\boldsymbol{x}}_{i, 2 n+1}\left(\boldsymbol{y}(t), \ldots, \boldsymbol{y}^{(n)}(t)\right) \\
& +\sum_{n=1}^{\infty}\left[1+\frac{2 n}{P e_{i}}\right] \hat{\boldsymbol{x}}_{i, 2 n}\left(\boldsymbol{y}(t), \ldots, \boldsymbol{y}^{(n-1)}(t), y_{i}^{(n)}(t)\right)=: \sum_{n=0}^{\infty} \hat{u}_{i, n}(t), \quad i=1,2
\end{aligned}
$$

genügen. Unter hier nicht näher spezifizierten, die Anwendung stark einschränkenden Bedingungen kann ein Konvergenzradius größer eins für (32) nachgewiesen werden, falls $\boldsymbol{y}(t)$ GevreyFunktionen jeweils der Ordnung $\alpha \leq 2$ und die Peclet-Zahlen $P e_{i} \leq 2, i=1,2$, sind (siehe

\footnotetext{
${ }^{4}$ Für eine ausführliche Betrachtung der Differenzialrekursion, Bestimmung der nicht eindeutigen parametrierenden Funktion und schematischen Parametrierung wird auf Kapitel 2.2.2 verwiesen.
} 
Kapitel 2.2.2). Dies bedeutet, dass zwar gleichmäßige Konvergenz in einem Bereich hoher Diffusion durch geeignete Trajektorienplanung gewährleistet werden kann, die Reaktoren jedoch in der Praxis im hoch-konvektiven Bereich mit $P e_{1} \approx 200$ bzw. $P e_{2} \approx 100$ betrieben werden. Diese Einschränkung gilt nur für die klassische Partialsumme, jedoch nicht für die $(N, \xi)-$ approximative $k$-Summe, deren Leistungsfähigkeit in Abb. 3 gezeigt wird. Hier werden numerische Ergebnisse für die Anwendung der mittels $(N, \xi)$-approximativer $k$-Summation entworfenen Randsteuerungen

$$
u_{i, k_{i}}^{N, \xi_{i}}(t)=\mathcal{S}_{k_{i}}^{N, \xi_{i}}\left(\sum_{n=0}^{\infty} \hat{u}_{i, n}(t)\right), \quad i=1,2
$$

mit geeigneten Summationsparametern $\xi_{i}$ und $k_{i}, i=1,2$, dargestellt. Als Simulationsmodell dienen hierbei die semi-diskretisierten Gleichungen des Rohrreaktors. Die Solltrajektorien $y_{i, d}(t), i=1,2$, sind so gewählt, dass ein Übergang zwischen vier verschiedenen stationären Profilen zum Anfahren, zwei Arbeitspunktwechseln und Abfahren realisiert werden kann (siehe Kapitel 4.1 und 4.4.2). Trotz einiger Abweichungen im Temperaturverlauf $x_{2}(0, t)$, die durch Approximationsfehler beim Steuerungsentwurf zu begründen sind, wird gutes Folgeverhalten erzielt. Insbesondere ist hierbei zu betonen, dass in der Literatur zur Regelung dieser Systeme üblicherweise nur Peclet-Zahlen $P e_{i}<10, i=1,2$, betrachtet werden (Christofides, 2001; Antoniades and Christofides, 2001).

Des Weiteren kann das für die lineare Wärmeleitungsgleichung vorgeschlagene Verfahren zum flachheitsbasierten Entwurf einer Folgeregelung mit Beobachter auf dieses nichtlineare Modell übertragen werden (siehe Kapitel 5.2.2). Hierzu zeigt Abb. 4 Simulationsergebnisse im Fall moderater $P e$-Zahlen. Zur Illustration der Robustheit der Folgeregelung wird die Da-Zahl im Simulationsmodell um 10\% höher als im Entwurfsmodell angenommen. Die Reaktion läuft somit exothermer ab als im nominellen Fall. Trotz dieses Modellfehlers kann die Folgeregelung das System (27)-(30) nahezu exakt auf die vorgegebenen Solltrajektorien führen.

Zusätzlich kann der anhand des Entwurfsmodells entworfene Beobachter für die Koeffizienten der formalen Potenzreihe zur Profilschätzung herangezogen werden. Im Prinzip werden hierzu die Beobachterzustände zur Bestimmung der betrachteten Reihenkoeffizienten herangezogen und entsprechend der Summationvorschrift addiert (siehe auch Kapitel 5.2.1.3). Wie Abb. 4 zeigt, erlaubt dies eine hochgenaue Bestimmung des Profils, wobei hier nur $\boldsymbol{x}(0, t)$ als Messgröße angenommen wurde.

\section{Zusammenfassung}

In dieser Arbeit werden die vielfältigen Möglichkeiten der Anwendung formaler Potenzreihen und geeigneter Summationsmethoden zum Steuerungs- und flachheitsbasierten Folgeregelungsentwurf für lineare und nichtlineare parabolische DKRGn erläutert. Die durchgängige Verwendung formaler Potenzreihen zur Systemparametrierung und Herleitung geeignter Entwurfsmodelle erlaubt einen systematischen Zugang, der direkt zur numerischen Auswertung und rechnerunterstützten Implementierung verwendet werden kann. Dabei werden die Ergebnisse der 

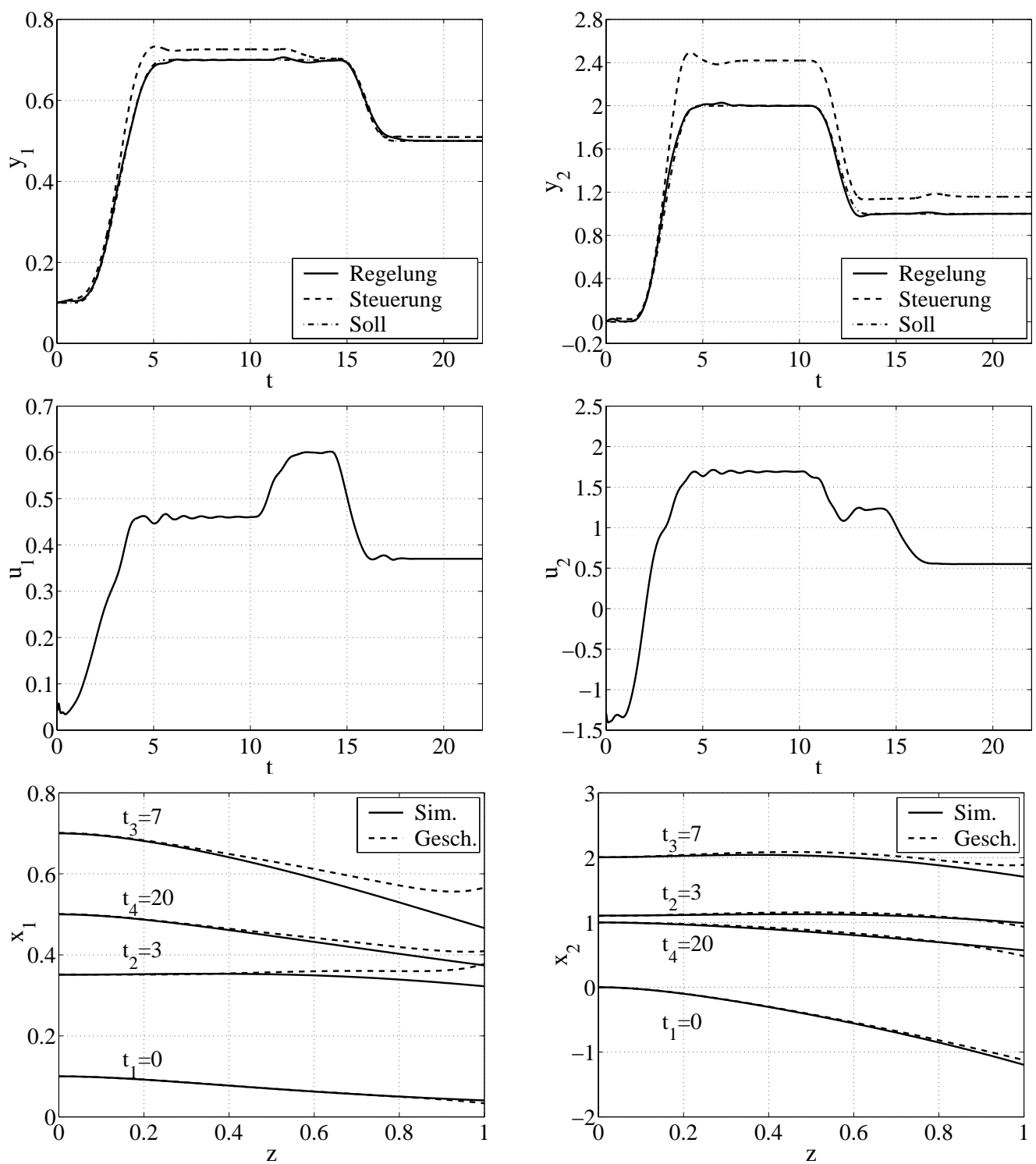

Bild 4: Numerische Simulation des Rohrreaktormodells (27)-(30) mit flachheitsbasierter Folgeregelung für $P e_{1}=P e_{2}=6, L e=1$ mit Modellfehler $D a_{\text {Reaktor }}=1.1 D a_{\text {Entwurf }}$ (weitere Parametern entsprechend Anhang D.2). Zusätzlich sind die Ergebnisse der reinen Steuerung dargestellt. Oben: Folgeverhalten für $x_{1}(0, t)$ and $x_{2}(0, t)$; Mitte: zeitlicher Verlauf der Eingriffsgößen; Unten: Vergleich der geschätzten Profile $\tilde{x}_{i}\left(z, t_{j}\right), i=1,2$ und simulierten Profile $x_{i}\left(z, t_{j}\right), i=1,2$ zu verschiedenen Zeitpunkten $t_{j}, j=1,2,3,4$.

Methodik anhand der linearen Wärmeleitungsgleichung, der linearen Diffusions-KonvektionsGleichung, skalarer nichtlinearen DKRGn mit polynomialen Nichtlinearitäten sowie einem komplexen Modell eines nicht-isothermen Rohrreaktors erläutert. Weitere Arbeiten betreffen u.a. die Anwendung formaler Potenzreihen in Kombination mit geeigneten Summationsmethoden zum Steuerungsentwurf für DKRGn mit Eingangsbeschränkungen (Meurer et al., 2005). 



\section{Chapter 1}

\section{Introduction}

The control of infinite-dimensional systems, i.e. systems described by delay equations or partial differential equations (PDEs), is an area of research comprising both theoretical impacts and practical applications stemming e.g. from mechanics or chemical engineering. Thereby the mathematical treatment of these control problems is complicated by their infinite-dimensional character.

In particular modeling of chemical processes leads to parabolic diffusion-convection-reaction equations (DCREs) due to the appearing diffusive and convective phenomena combined with strong nonlinearities in the reactive regime. Well-known examples concern tubular and fixedbed reactors with multiple steady states and complex dynamical behavior (Jensen and Ray, 1982), where in addition to the stabilization problem the increasing demands on product quality and process efficiency require advanced model-based tracking control schemes to force the output variables to follow some pre-defined trajectories. This illustrates the importance of appropriate motion planning, i.e. the design of feasible output trajectories. Typical examples concern startup or shutdown of a reactor, as well as the realization of finite-time transitions between stationary operating points. Although being of practical interest, traditional approaches seem to provide only indirect and often unsatisfactory solutions to the tracking control problem.

\subsection{Control of systems governed by PDEs}

Model-based control design methods for DCREs are typically based on either early or late lumping approaches. In the early lumping approach, the process model is approximated first, using for instance finite-difference or finite-element techniques, modal approaches (Georgakis et al., 1977a,b,c; Balas, 1978), proper orthogonal decompositions (Atwell and King, 2001), or Galerkin's method and its modifications (Ray, 1981; Christofides, 2001), in order to proceed with the controller synthesis based on the approximation of the infinite-dimensional system by a finite-dimensional set of ordinary differential equations (ODEs) capturing the essential dynamics. Nevertheless, while being useful for system analysis, these approximations usually lead to high-dimensional and often complex model-based feedback control structures, confining 
their applicability. Furthermore an immense computational effort is often required for the determination of the finite-dimensional process models, as e.g. in the case of Galerkin's method in conjunction with so-called (approximate) inertial manifolds (Temam, 1988; Christofides, 2001).

In the late lumping approach, the distributed nature of the system is kept as long as possible and controller synthesis is performed using the infinite-dimensional process model. Thereby, the functional analytic setting using semigroup theory and related concepts (see Curtain and Zwart (1995) for a modern and comprehensive overview) has proven to be a powerful tool to feedback control design for delay equations, parabolic and also hyperbolic distributed-parameter systems (DPS). If the control is applied spatially concentrated, e.g. at a boundary of a given plant, the application of semigroup theory requires a reformulation of the control problem into an abstract boundary control system as initially introduced by Fattorini (1968). For the boundary control of parabolic PDEs, various general results exist concerning the design of feedback control, see e.g. the extensive work of Nambu $(1979,1984)$ or Lasiecka and Triggiani (1983) in the functional analytic setting or the comprehensive treatise in Curtain and Zwart (1995). The main drawback of this theoretically appealing approach stems from the necessary approximation of the usually obtained infinite-dimensional control laws as required for implementation. In addition, the extension to nonlinear DPSs is not straightforward.

It is particularly interesting to notice that besides optimal control for PDEs (Butkovsky, 1969; Lions, 1971; Ray, 1981; Fursikov, 1999) with their computational drawbacks, the main focus of controller design concerns the stabilization problem. Only few analytical (late lumping) approaches exist to the design of tracking control for DPS, i.e. control of a fixed plant so that its outputs track desired reference signals. In the functional analytic setting, initial results on the output tracking problem for DPS by means of feedback boundary control are provided in Byrnes et al. $(2000,2004)$, who extend the geometric theory of output regulation to linear infinitedimensional control systems. Nevertheless in general, there is no straightforward extension of results from finite-dimensional control theory to infinite dimensions.

\section{$1.2 \quad$ Flatness-based methods for PDEs}

On the other hand when dealing with tracking control problems for finite-dimensional linear and nonlinear systems, differential flatness (Fliess et al., 1995; Rothfuß et al., 1996; Rothfuß, 1997; Rothfuß et al., 1997) is a well established tool for system analysis, motion planning, openloop as well as feedback control design. The recent work on flatness has mainly dealt with its extension to motion planning and open-loop control design for infinite-dimensional systems in the late-lumping context, i.e. it is desired to directly study the governing infinite-dimensional system (Rudolph, 2003a). It was shown, that the type of PDE (parabolic or hyperbolic) determines the approach for parameterizing the solution by a flat output, such that there is no general definition of differential flatness for DPS. However, the property of parameterizability can be interpreted as the underlying principle as is illustrated below. Therefore, consider a 
scalar second order PDE in the state $x(z, t)$ with distributed, infinite-dimensional input $u(z, t)$, i.e.

$$
\frac{\partial x(z, t)}{\partial t}=G\left(x(z, t), \frac{\partial x(z, t)}{\partial z}, \frac{\partial^{2} x(z, t)}{\partial z^{2}}\right)+u(z, t), \quad z \in(0,1), t>0
$$

and boundary (BCs) and initial conditions (IC)

$$
\begin{gathered}
R_{j}\left(x(j, t), \frac{\partial x}{\partial z}(j, t)\right)=0, \quad t>0, \quad j=0,1 \\
x(z, 0)=x_{0}(z), \quad z \in[0,1] .
\end{gathered}
$$

Obviously the choice $y(z, t)=x(z, t)$ provides the inverse system representation

$$
x(z, t)=y(z, t), \quad u(z, t)=\frac{\partial y(z, t)}{\partial t}-G\left(y(z, t), \frac{\partial y(z, t)}{\partial z}, \frac{\partial^{2} y(z, t)}{\partial z^{2}}\right)
$$

parameterized in terms of $y(z, t)$ and its derivatives. In view of Fliess et al. (1995), the function $y(z, t)$ can be interpreted as a flat or parameterizing output. Hence by specifying a trajectory $(z, t) \mapsto y_{d}(z, t)$ being consistent with the BCs and IC, substitution of $y_{d}(z, t)$ into the latter equation directly yields the feedforward control $u_{d}(z, t)$ necessary to track the state $x(z, t)$ along $x_{d}(z, t)=y_{d}(z, t)$ in the nominal case, i.e. exponentially stable system without model errors or disturbances. Obviously this is a problem of motion planning in view of the BCs and IC followed by purely algebraic operations to determine $u_{d}(z, t)$. In case of an unstable system, the feedback

$$
u(z, t)=\frac{\partial y_{d}(z, t)}{\partial t}-p_{0}\left[y(z, t)-y_{d}(z, t)\right]-G\left(y(z, t), \frac{\partial y(z, t)}{\partial z}, \frac{\partial^{2} y(z, t)}{\partial z^{2}}\right)
$$

provides a linear PDE for the tracking error $e(z, t)=y(z, t)-y_{d}(z, t)$, i.e.

$$
\frac{\partial e(z, t)}{\partial t}=-p_{0} e(z, t), \quad z \in(0,1), t>0
$$

which is asymptotically stable for any $p_{0}>0$. Hence in the case of infinite-dimensional input functions $u(z, t)$, the flatness results for finite-dimensional systems directly carry over to the infinite-dimensional case. Nevertheless these appealing results are not reflected in applications where typically the input is either located at the boundaries, i.e. $R_{j}(x(j, t), \partial x / \partial z(j, t))=$ $u_{j}(t), j=0,1$ or finitely distributed along the spatial domain, i.e. $u(z, t)=\sum_{j=1}^{m} b_{j}(z) u_{j}(t)-$ both significantly complicate analysis and design. However under certain assumptions on the structure of the PDE, it is still possible to determine the inverse system representation for such DPS control systems in a fashion similar to the above.

Applying operational calculus or series approaches, for boundary controlled parabolic and biharmonic PDEs the parameterization of system states and input by a flat or parameterizing output leads to fractional differentiation operators or respectively infinite power series representations (Fliess et al., 1997; Rudolph and Woittennek, 2002). The series coefficients depend on 
time-derivatives of a flat output, which has to be chosen to belong to a certain Gevrey class to ensure convergence. The latter basically states that only smooth trajectories can be assigned, whose derivatives satisfy certain growth bounds.

Applications for parabolic PDEs concern motion-planning and open-loop boundary control for the linear heat equation (Martin et al., 1997; Laroche et al., 1998; Laroche and Martin, 2000; Laroche et al., 2000; Lynch and Rudolph, 2000a) and the linear diffusion equation (Laroche and Martin, 2000; Laroche et al., 2000; Lynch and Rudolph, 2000a, 2002) with spatially dependent coefficients (Laroche et al., 1998, 2000; Lynch and Rudolph, 2002) in several state-variables (Fliess et al., 1998a,b). Special work concerns cylindrical coordinate descriptions of a heated ring (Laroche and Martin, 2000) and Maxwell's equations modeling a solenoid valve (Rothfuß et al., 2000). Work on time as well as state dependent coefficients is available (Lynch and Rudolph, 2000a, 2002), considering scalar heat and diffusion equations. Finally, extensions to nonlinear parabolic equations with boundary control are provided by Lynch and Rudolph (2000a,b,c, 2002) for tubular reactor models of various complexity. A review on recent results on motion planning can be found in Rudolph (2004). In addition to the theoretical work cited above, experimental results validating the applicability of the flatness-based approach are available for motion planning and open-loop boundary control of a flexible robot arm modeled by the biharmonic Euler-Bernoulli equations (Aoustin et al., 1997). Further work considers hyperbolic PDEs with boundary inputs (Rudolph, 2000b, 2003a; Woittennek and Rudolph, 2003; Rouchon, 2005) and differing approaches for flatness-based open-loop control of nonlinear infinite-dimensional systems (Petit, 2002; Ollivier and Sedoglavic, 2001; Rouchon, 2001). For the design of spatially distributed control within the flatness concept, only few results are available for linear DPS. These are either based on certain assumptions on the input characteristics (Rudolph and Woittennek, 2002, 2003) or on the parameterization of the governing equations in modal states (Meurer, 2003a), (Meurer and Zeitz, 2003a).

However, within these results several questions remain open, which are directly related to the existing approaches and, as is illustrated, their evolving drawbacks.

\subsection{Goals of the thesis}

In this thesis, formal power series are applied for the design of feedforward and stabilizing feedback tracking control for boundary controlled linear and nonlinear DCR-systems in one spatial dimension. Thereby, it is desired to analyze the drawbacks of the existing approaches to feedforward boundary control design based on formal power series. These drawbacks can be summarized as follows:

- Motion planning for the parameterizing function is restricted to smooth functions of a certain Gevrey class to ensure series convergence. Numerical experiments for the linear heat equation (Laroche et al., 2000) show that this class can be prolonged, with the drawback of divergent series solutions, which have to be summed appropriately. 
- The proof of convergence does not yield any information on the respective speed of convergency, which might be slow for convection dominated systems (Wagner et al., 2004).

- In case of boundary controlled nonlinear DCR-systems, the resulting convergence conditions impose further bounds on the system parameters (Lynch and Rudolph, 2002), which severely limit the applicability of the approach (Meurer and Zeitz, 2005).

In order to overcome these limitations, the following fundamental question arises:

- Does there exist a uniform mathematical framework in order to deal with divergent series and convergence acceleration in view of motion planning and feedforward control design for nonlinear systems of DCR-equations with boundary inputs?

Besides being useful for motion planning and feedforward control design for nonlinear finitedimensional systems, the property of differential flatness directly allows the design of asymptotically stabilizing feedback tracking control (Fliess et al., 1995; Rothfuß, 1997). As outlined in the previous section, this property carries over to the case of flat or parameterizable DPS with infinite-dimensional inputs. Nevertheless, there is no analogon for parameterizable boundary controlled DPS. Consequently, the question arises:

- Using formal power series, is it possible to design flatness-based asymptotically stabilizing tracking control for nonlinear systems of DCR-equations?

It is known that the structure of the governing PDE system with BCs and ICs determines the possibility of parameterization. For benchmark examples such as the linear heat and diffusion equation, the choice of the parameterizing function is typically apparent. On the other hand, for general boundary controlled DCR-systems no criteria are available to decide whether the system is parameterizable in the described sense:

- Given a general boundary controlled DCR-system, is it possible to determine the parameterizing functions directly from the DCR structure? In other words, which real world examples are structurally parameterizable?

Besides the existence of a state and input parameterization, its explicit determination is desired in terms of a parameterizing function. This requires the solution of a differential recursion. Thereby, closed-form solutions are only available for particular linear recursions (e.g. the heat equation), and nonlinear problems only allow the determination of a certain number of series coefficients depending on the computational complexity. Since the quality of the parameterizations (at least for convergent series) is directly correlated to the number of series coefficients, appropriate computational algorithms are needed to maximize this number:

- What is the computational effort for the parameterization of the series coefficients in terms of the parameterizing function for real world problems? How can the necessary computations be realized using computer-algebra systems? 
In this thesis, it is desired to address these problems based on theoretical and numerical results. Therefore, several DCR examples such as the linear heat equation or the model of a nonisothermal fixed-bed tubular reactor are studied within the formal power series approach. Whenever possible, extensions are provided in order to generalize the underlying ideas.

\subsection{Structure of the thesis}

The structure of this thesis is can be directly deduced from the goals quoted in the previous section. For the feedforward control design, formal power series in the spatial variable with coefficients in some space of functions are applied in Chapter 2. Under the formal assumption of uniform series convergence, differential recursions for the series coefficients can be derived from the governing distributed-parameter descriptions. Evaluation of the recursions is possible whenever a sufficient number of starting conditions can be determined from the system's BCs. Thereby, additional BCs containing fictious, but typically physically interpretable, functions have to be imposed, which replace the inhomogeneous BCs involving the input variables. This allows the sequential computation of the series coefficients in terms of the imposed BCs and their time-derivatives up to infinite order. Uniform convergence of the resulting state parameterizations can be ensured by appropriate motion planning for the imposed BCs in terms of functions of certain Gevrey classes. Substitution of the arising parameterized uniformly convergent power series into the inhomogeneous BCs involving the inputs provides the input parameterization. Hence, an appropriate choice of trajectories for the imposed BCs directly yields the feedforward control necessary to track the prescribed trajectory in the nominal case, i.e. stable system without model errors or exogenous disturbances.

Nevertheless, certain restrictions emerge from the proof of convergence, which in various cases prevent the application of the determined series solutions. Especially when dealing with convective effects, which typically arise in applications governed by DCR-equations, the speed of convergence of the resulting series might possibly be very slow as is illustrated for the linear diffusion equation. In addition, nonlinear effects further restrict convergence to certain system parameter sets depending on the choice of trajectories for the imposed BCs. For nonlinear scalar DCR-equations, the parameter space of convergence characteristically shrinks when increasing convection, contracts to a single point at a certain threshold of convection, and vanishes afterwards. These effects are intensified when considering boundary controlled systems of DCR-equations.

Hence, a more general approach would be desirable to extract meaningful expressions from the determined formal series solutions. Although rather surprising, it is shown in Chapters 3 and 4 , that divergent series solutions provide the mathematical framework to overcome these restrictions. Therefore, Chapter 3 summarizes the necessary mathematical tools for the study of (divergent) formal power series with coefficients in Banach spaces. In doing so, the definitions, results, and examples are arranged in view of the introduction of summation methods for power series with particular focus on $k$-summation. For its application and implementation, 
a variant, the so-called $(N, \xi)$-approximate $k$-summation, is defined in order to account for convergence acceleration and summation of divergent series in cases when only a finite number of series coefficients is available. Note that this situation is typical for the formal parameterization of boundary controlled DCR-systems. Since the summation of divergent series is also a common tool in quantum physics (Zinn-Justin, 1989) and quantum chemistry (Weniger, 1994), prototype examples of so-called generalized sequence transformations used in these branches are studied for comparison purposes.

After general remarks on motion planning for DCR-systems, the results of Chapter 3 are exemplarily applied to motion planning and feedforward control design for linear and nonlinear parabolic DPS in Chapter 4. Thereby, the properties of the different summation techniques are compared for summing divergent series solutions of the linear heat equation and for convergence acceleration of convergent series solutions of the linear diffusion-convection equation with increasing convective effects. Based on the results for these simple examples, it is shown that the introduced $(N, \xi)$-approximate $k$-summation provides a widely applicable approach. This is confirmed by results for the feedforward control of a non-isothermal tubular reactor model, where the control design can be performed for a broad range of system parameters covering the unpacked as well as the packed fixed-bed reactor with severe dynamical effects. Some generalizations conclude the chapter.

Based on the promising results for motion planning and feedforward tracking control design for boundary controlled nonlinear DCR-systems using formal power series and suitable summation techniques, Chapter 5 is devoted to the design of flatness-based feedback tracking control with observer. Therefore, it is shown that a re-interpretation of the formal power series approach allows to determine an inherently flat, finite-dimensional nonlinear control system in the power series coefficients, which approximates the originally infinite-dimensional structure. In particular, application of the $(N, \xi)$-approximate $k$-summation technique provides a further increase in the quality of the approximation. For the finite-dimensional approximation, classical flatness-based methods can be utilized for tracking control design. Since full state information is necessary for the implementation of the flatness-based tracking control, an observer is needed to estimate the non-measured states. As is shown, the designed observer can be additionally used for spatial profile estimation during the transition process to provide further insight in the process behavior e.g. for monitoring purposes. Finally, the results of the thesis are summarized in Chapter 6.

In Appendix A, some mathematical background is provided, followed by a brief introduction of smooth functions with compact support in Appendix B. As outlined above, state and input parameterization is based on the solution of a differential recursion, where closed-form expressions are only available in special cases. For nonlinear problems, one is restricted to sequentially evaluate the recursion formula to determine a certain number of series coefficients. Thereby, the possible number of series coefficients is limited by the computational effort. In Appendix $\mathrm{C}$ an algorithm is proposed which allows to symbolically compute a large number of series coefficients using computer-algebra systems (CAS). Lemmas and parameters used for the considered non-isothermal tubular reactor example are summarized in Appendix D. 


\section{Chapter 2}

\section{Formal power series parameterization of boundary controlled DCR-systems}

In this chapter, the parameterization of diffusion-convection-reaction equations with boundary inputs using formal power series is studied for various system structures. Starting from the linear heat and diffusion-convection equation, nonlinear scalar as well as systems of DCRequations are analyzed and studied within numerical simulations. Thereby, uniform convergence of the resulting series has to be ensured by appropriate motion planning.

This crucial step within the analysis is carried out exemplarily for the linear heat and diffusionconvection equation as well as the case of polynomial nonlinearities in Section 2.1. Thereby, emerging drawbacks are illustrated which appear within the classical framework of uniformly convergent power series. These serve as a motivation to embed the formal power series results into a larger class of series containing only pointwise convergent and certain divergent series. Based on results for MIMO DCR-equations in Section 2.2, the new notion of formal power series parameterizability is motivated and defined for general boundary controlled DCR-systems in Section 2.3. This notion basically states that any system state and input of a formally parameterizable DCR-system can be expressed in terms of parameterizing functions (or basic outputs) and their time-derivatives possibly up to an infinite order. For DCR-systems satisfying these conditions, the resulting formal parameterizations can be directly utilized for feedforward control design.

\subsection{Scalar DCR-equations with boundary input}

In the sequel, the application of formal power series to analyze boundary controlled DCRsystems is motivated by studying several variants of scalar DCR-equations, which typically arise in chemical and biochemical engineering. Thereby, formal power series are used to determine an inverse system representation in view of feedforward control design. 
As a general scalar example consider the following nonlinear DCR-equation

$$
\frac{\partial x(z, t)}{\partial t}=\lambda \frac{\partial^{2} x(z, t)}{\partial z^{2}}+\nu \frac{\partial x(z, t)}{\partial z}+\beta x(z, t)+\Psi(x(z, t)), \quad z \in(0,1), t>0
$$

with initial condition (IC)

$$
x(z, 0)=x_{0}(z), \quad z \in[0,1]
$$

and consistent boundary conditions (BCs)

$$
\begin{aligned}
\frac{\partial x}{\partial z}(0, t) & =0, \quad t>0 \\
p_{1} \frac{\partial x}{\partial z}(1, t)+r_{1} x(1, t) & =u(t), \quad t>0 .
\end{aligned}
$$

It is assumed that convection takes place in the negative $z$-direction, such that $\nu>0$. Furthermore, state $x(z, t)$, input $u(t)$, and any system parameter are considered as non-dimensional, which can be achieved by normalization.

By suitably choosing the system parameters $\lambda, \nu, \beta, p_{1}$, and $r_{1}$ as well as the nonlinearity $\Psi(x(z, t))$, several well-known boundary controlled DPS examples can be derived from (2.1)(2.4). At first two linear versions of (2.1) are studied, i.e. $\Psi(x) \equiv 0$, namely the linear heat equation $(\nu=0)$ and the linear diffusion-convection equation $(\nu>0)$. Secondly, the nonlinear scalar DCR-equation is considered for polynomial nonlinearities $\Psi(x)$.

\subsubsection{The linear heat equation}

In the special case of $\Psi(x) \equiv 0$ and $\nu=0,(2.1)-(2.4)$ reduce to the boundary controlled linear heat equation. Applying the Laplace transformation or, quite similar but in a more general framework of convolution, the so-called Mikusiński transformation (Mikusiński, 1983; Mikusiński and Boehme, 1987), the solution to the initial-boundary-value-problem (IBVP) can be easily determined. For Laplace as well as Mikusiński transformation, the heat equation (2.1) can be associated with the following $s$-dependent boundary value problem (BVP) for the ordinary differential equation (ODE)

$$
\begin{aligned}
& \lambda \frac{d^{2} X(z, s)}{d z^{2}}=(s-\beta) X(z, s), \quad z \in(0,1) \\
& \frac{d X}{d z}(0, s)=0 \\
& p_{1} \frac{d X}{d z}(1, s)+r_{1} X(1, s)=U(s)
\end{aligned}
$$

assuming $x_{0}(z) \equiv 0$, which can be achieved for any stationary initial profile. Note that the case $x_{0}(z) \not \equiv 0$ can be treated similarly. Here, $X(z, s)$ and $U(s)$ denote the operational function corresponding to $x(z, t)$ and $u(t)$, respectively. The general solution to (2.5)-(2.7) can be expressed as

$$
X(z, s)=\frac{\cosh (\kappa(s) z)}{r_{1} \cosh (\kappa(s))+\kappa(s) p_{1} \sinh (\kappa(s))} U(s)
$$


where $\kappa(s)=\sqrt{(s-\beta) / \lambda}$. The solution $x(z, t)$ in the original time-domain can be determined by applying the inverse Laplace (or Mikusiński) transformation on (2.8) for given $U(s)$. On the other hand from a control perspective, it is desired to determine $U(s)$ or respectively $u(t)$, to solve a certain control problem. Therefore, rewrite (2.8) in the following two-step procedure introducing a new quantity $Y(s)$

$$
\begin{aligned}
U(s) & =D(s) Y(s) \quad \text { with } \quad D(s)=\left[r_{1} \cosh (\kappa(s))+\kappa(s) p_{1} \sinh (\kappa(s))\right] \\
X(z, s) & =N(z, s) Y(s) \quad \text { with } \quad N(z, s)=\cosh (\kappa(s) z) .
\end{aligned}
$$

Equation (2.8) can be recovered by solving either equation for $Y(s)$ and substituting the result into the remaining equation. This approach allows further interpretation by evaluation of (2.10): substituting $z=0$ provides $X(0, s)=Y(s)$. Hence in view of $(2.9)$ and $(2.10), U(s)$ and $X(z, s)$ can be expressed in terms of $Y(s)=X(0, s)$. This provides an operational parameterization of input $U(s)=D(s) Y(s)$ and state $X(z, s)=N(z, s) Y(s)$ in terms of $Y(s)$.

By re-writing $D(s)$ and $N(z, s)$ using series expansions of hyperbolic functions (Bronstein et al., 1997), it follows that

$$
\begin{aligned}
U(s) & =\left[r_{1} \sum_{n=0}^{\infty} \frac{(s-\beta)^{n}}{\lambda^{n}(2 n) !}+p_{1} \sum_{n=0}^{\infty} \frac{(s-\beta)^{n+1}}{\lambda^{n+1}(2 n+1) !}\right] Y(s) \\
X(z, s) & =\sum_{n=0}^{\infty} \frac{(s-\beta)^{n} z^{2 n}}{\lambda^{n}(2 n) !} Y(s) .
\end{aligned}
$$

Furthermore, termwise application of the inverse transformation with the correspondence $s^{n} \bullet \bullet \frac{d^{n}}{d t^{n}}$ for any $n \in \mathbb{N}$ together with the binomial theorem yields the parameterization of $u(t)$ and of $x(z, t)$ in terms of $y(t)=x(0, t)$, i.e.

$$
\begin{aligned}
u(t)= & r_{1} \sum_{n=0}^{\infty} \frac{1}{\lambda^{n}(2 n) !} \sum_{i=0}^{n}\left(\begin{array}{c}
n \\
i
\end{array}\right)(-\beta)^{n-i} y^{(i)}(t)+ \\
& p_{1} \sum_{n=0}^{\infty} \frac{1}{\lambda^{n+1}(2 n+1) !} \sum_{i=0}^{n+1}\left(\begin{array}{c}
n+1 \\
i
\end{array}\right)(-\beta)^{n+1-i} y^{(i)}(t) \\
x(z, t)= & \sum_{n=0}^{\infty} \frac{z^{2 n}}{\lambda^{n}(2 n) !} \sum_{i=0}^{n}\left(\begin{array}{c}
n \\
i
\end{array}\right)(-\beta)^{n-i} y^{(i)}(t) .
\end{aligned}
$$

Meaningful expressions for $x(z, t)$ and hence $u(t)^{1}$ can be recovered from the formal solutions, if a radius of convergence $\rho \geq 1$ can to be verified for (2.14). This is sufficient since the set of power series being uniformly convergent for $z \in[0, \rho)$ and $t \in \mathbb{R}^{+}$(or $t \in[0, T], T>0$ ) forms a differential algebra, such that differentiations with respect to $z$ and $t$ become valid operations preserving convergency. In view of (2.14), this is obviously a problem of finding appropriate functions $y(t)$, which first have to be of class $\mathcal{C}^{\infty}$, i.e. smooth, and secondly satisfy certain growth conditions on their derivatives. This problem is well-known - see e.g. (Widder, 1975, p.51) or (Taylor, 1996, p.225) — and the convergence results reads as follows:

\footnotetext{
${ }^{1}$ The expression for $u(t)$ can be determined from BC $(2.4)$ by differentiating $x(z, t)$. It hence suffices to prove uniform convergence for (2.14) to validate this operation.
} 
Theorem 2.1. The series (2.14) converges to the solution of (2.1) with $\nu=0$ and BCs (2.3), (2.4) for all $z$, i.e. the series has an infinite radius of convergence, if there exist $M, R \in \mathbb{R}^{+}$ such that $y(t)$ satisfies

$$
\sup _{t \in \mathbb{R}^{+}}\left|y^{(n)}(t)\right| \leq \frac{M}{R^{n}}(n !)^{\alpha}, \quad n \in \mathbb{N}_{0}
$$

for $\alpha<2$.

Functions $y(t)$ satisfying condition (2.15) are called Gevrey of order $\alpha$. This notion will be re-considered in Chapter 3.

Proof. Assume that (2.15) holds, then the inner sum of (2.14) satisfies

$$
\begin{aligned}
\left|\sum_{j=0}^{n}\left(\begin{array}{c}
n \\
j
\end{array}\right)(-\beta)^{n-j} y^{(j)}(t)\right| & \leq M|\beta|^{n}(n !)^{\alpha+1} \sum_{j=0}^{n} \frac{1}{(|\beta| R)^{j} j !(n-j) !|\beta|^{j}} \\
& \leq M\left(\frac{1+R|\beta|}{R}\right)^{n}(n !)^{\alpha}=\frac{M}{\widetilde{R}^{n}}(n !)^{\alpha} .
\end{aligned}
$$

Hence the Gevrey order $\alpha$ is preserved within this inner sum. Substituting

$$
\widetilde{y}(t):=\sum_{j=0}^{n}\left(\begin{array}{l}
n \\
j
\end{array}\right)(-\beta)^{n-j} y^{(j)}(t)
$$

in (2.14) and applying the Cauchy-Hadamard Theorem A.1 outlined in Appendix A, the radius of convergence $\rho$ of the series (2.14) can be determined by

$$
\frac{\lambda}{\rho^{2}}=\limsup _{n \rightarrow \infty}\left|\frac{\widetilde{y}(t)}{(2 n) !}\right|^{\frac{1}{n}} \stackrel{(2.16)}{\leq} \limsup _{n \rightarrow \infty} \frac{1}{\widetilde{R}} \frac{\left[n !^{\frac{1}{n}}\right]^{\alpha}}{\left[(2 n) ! \frac{1}{2 n}\right]^{2}} \leq \limsup _{n \rightarrow \infty} \frac{1}{4 \widetilde{R}}\left(\frac{n}{e}\right)^{\alpha-2}
$$

where the last inequality can be deduced from Stirling's formula summarized in Lemma A.1 of the Appendix. As a result, it follows that

$$
\rho= \begin{cases}\infty & \text { for } \alpha<2 \\ 2 \sqrt{\lambda \widetilde{R}} & \text { for } \alpha=2 \\ 0 & \text { for } \alpha>2\end{cases}
$$

As a main result of the above analysis, suitably chosen functions $y(t)=x(0, t)$ provide the feedforward control $u(t)$ by evaluating (2.13), which when applied to the governing heat equation $(2.1)-(2.4)$ yields the solution $x(0, t)=y(t)$. Hence, $u(t)$ can be interpreted as the feedforward control to ensure tracking of the trajectory $t \mapsto y(t)$ in open-loop mode.

In order to illustrate the result of Theorem 2.1, a function $t \mapsto y_{d}(t)$ of class $\mathcal{C}^{\infty}$ satisfying condition (2.15) has to be assigned for $y(t)$. Under the assumption of a desired finite-time 
transition between stationary profiles during $t \in[0, T], y_{d}(t)$ naturally has to be non-analytical at the initial and final point. This is due to Liouville's theorem (Rudin, 1999, p. 253) since an analytic function being constant on an interval of its domain is necessarily constant on the whole domain. As outlined in detail in Section 4.1.1, stationary profiles for the heat equation are implicitly parameterized by the determined series (2.14) when considering the limit $t \rightarrow \infty$ such that $y(t) \rightarrow y_{S}, y^{(n)}(t) \rightarrow 0$ for $n \geq 1$. Hence changes in $y_{S}$ result in different stationary profiles.

A prototype function (B.1) fulfilling these requirements is studied in Appendix B and used for the following numerical evaluations. Since convergence is ensured by Theorem 2.1, it is sufficient to sum only a finite number of terms, i.e.

$$
\begin{aligned}
u_{N}(t)= & r_{1} \sum_{n=0}^{N} \frac{1}{\lambda^{n}(2 n) !} \sum_{i=0}^{n}\left(\begin{array}{c}
n \\
i
\end{array}\right)(-\beta)^{n-i} y^{(i)}(t)+ \\
& p_{1} \sum_{n=0}^{N-1} \frac{1}{\lambda^{n+1}(2 n+1) !} \sum_{i=0}^{n+1}\left(\begin{array}{c}
n+1 \\
i
\end{array}\right)(-\beta)^{n+1-i} y^{(i)}(t)
\end{aligned}
$$

in order to approximate the function $u(t)$. This is motivated by the fact that for given $\epsilon>0$ there always exists an $N_{\epsilon} \in \mathbb{N}$ such that $\left|u(t)-u_{N}(t)\right| \leq \epsilon$ for $N \geq N_{\epsilon}$ due to convergence. Substituting $y(t)$ by $y_{d}(t)=\Phi_{\gamma, T}(t)$ defined in (B.1), $u_{N}(t)$ yields the necessary feedforward control to track the quantity $y(t)=x(0, t)$ of the DPS (2.1)-(2.4) with $\nu=0$ and $\Psi(x)=0$ along $y_{d}(t)$ in open-loop. Numerical results when varying the Gevrey order $\alpha$ of $y_{d}(t)$ with system parameters $\lambda=1, \beta=0, p_{1}=0, r_{1}=1$ are depicted in Figure 2.1. In order to compare the obtained behavior for $y(t)=x(0, t)$ with its desired value $y_{d}(t)$, the feedforward control $u_{N}(t)$ for $N=20$ is applied to a 'method-of-lines' (MOL) discretized simulation model of (2.1)-(2.4). Obviously no deviations between actual and desired behavior appear for Gevrey orders $\alpha \leq 2$. From the simulation results, a decrease in input amplitude can be observed for increasing $\alpha$. This is due to the declining amplitude of the gradients of $y_{d}(t)$ whenever $\alpha$ is increased as outlined in Appendix B.

On the other hand when increasing $\alpha$ above the verified threshold of $\alpha=2$, spurious oscillations appear at the boundaries of the temporal domain $t \in[0, T]$. Nevertheless, for $\alpha>2$ but close to the threshold 2 from Theorem 2.1, actual and desired behavior match nicely whereby a much smoother transition is obtained ${ }^{2}$. Hence it seems promising to consider also the divergent case, whereby classical summation must be replaced by stronger techniques as will be shown in the sequel. Initial results on this topic can be also found in Laroche et al. (2000).

Compared to the application of operational calculus with its necessity to invert the solution operators, the previous results illustrate that it is alternatively possible to directly analyze the governing PDE in the time domain using a formal power series. The notion formal simply denotes the fact, that the radius of convergence of the power series might eventually equal zero.

\footnotetext{
${ }^{2}$ Note that this result depends also on the number $N$ of series coefficients used for partial summation. By increasing $N$ above the used value of $N=20$, spurious oscillations might appear.
} 

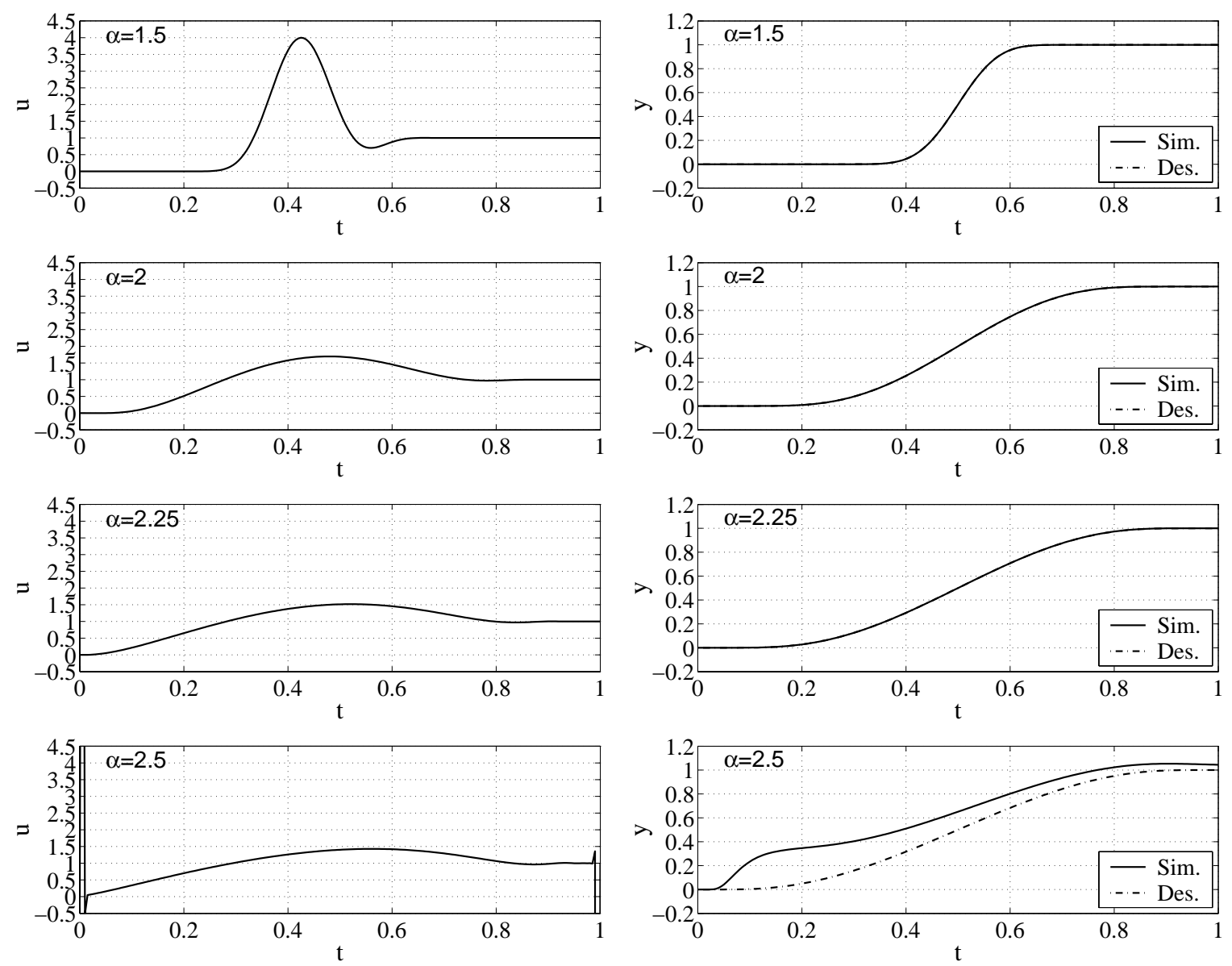

Figure 2.1: Comparison of numerical results for open-loop boundary control of (2.1)-(2.4) with $\lambda=1$, $\nu=0, \beta=0, p_{1}=0, r_{1}=1, \Psi(x)=0$ for desired trajectory $y_{d}(t)=\Phi_{\gamma, T}(t)$ defined in (B.1) with $T=1$ when varying $\gamma$ or $\alpha=1+1 / \gamma$, respectively. Left: feedforward control $u_{N}(t)$ from (2.17) with $N=20$; right: output trajectory $y(t)=x(0, t)$ compared to desired trajectory $y_{d}(t)$.

In order to illustrate this, introduce

$$
\begin{aligned}
\hat{X}_{2 n}(s) & =\frac{(s-\beta)^{n}}{\lambda^{n}(2 n) !} Y(s) \\
\hat{X}_{2 n+1}(s) & =0 .
\end{aligned}
$$

Hence, Eqn. (2.12) can be rewritten as

$$
\hat{X}(z, s)=\sum_{n=0}^{\infty} \hat{X}_{n}(s) z^{n}
$$

which reads in the time-domain

$$
\hat{x}(z, t)=\sum_{n=0}^{\infty} \hat{x}_{n}(t) z^{n} .
$$


In view of (2.18), (2.19) and $s^{n} \bullet-\frac{d^{n}}{d t^{n}}, n \in \mathbb{N}_{0}$, the time-varying coefficients of (2.20) evaluate to

$$
\begin{aligned}
\hat{x}_{2 n}(t) & =\frac{1}{\lambda^{n}(2 n) !} \sum_{i=0}^{n}\left(\begin{array}{c}
n \\
i
\end{array}\right)(-\beta)^{n-i} y^{(i)}(t) \\
\hat{x}_{2 n+1}(t) & =0 .
\end{aligned}
$$

Furthermore, it follows from (2.12) that

$$
\hat{U}(s)=r_{1} \sum_{n=0}^{\infty} \hat{X}_{n}(s)+p_{1} \sum_{n=0}^{\infty}(n+1) \hat{X}_{n+1}(s)
$$

or respectively

$$
\hat{u}(t)=\sum_{n=0}^{\infty}\left(p_{1}(n+1) \hat{x}_{n+1}(t)+r_{1} \hat{x}_{n}(t)\right) .
$$

The previous analysis illustrates, that it is equivalent to study the governing (linear) PDE using operational calculus or start with a formal power series in the spatial coordinate with time-varying coefficients. As will be illustrated in Section 2.2, the latter approach is more powerful since it also allows to treat special classes of nonlinear PDEs. Before doing so, a second prototype example, namely the linear diffusion-convection equation, is studied using the formal power series approach.

\subsubsection{The linear diffusion-convection equation}

The general scalar DCR-equation (2.1)-(2.4) reduces to the boundary controlled linear diffusion-convection equation for $\Psi(x) \equiv 0$. Although this PDE can be converted into the linear heat equation by introducing the transformation

$$
\tilde{x}(z, t):=x(z, t) \exp \left(\frac{\nu}{2 \lambda} z\right)
$$

the original problem is considered in order to illustrate the convective effect on the results obtained when studying a formal ${ }^{3}$ solution in terms of a power series ${ }^{4}$

$$
\hat{x}(z, t)=\sum_{n=0}^{\infty} \hat{x}_{n}(t) z^{n} .
$$

\footnotetext{
${ }^{3}$ The term formal is used to express that any operation such as differentiation (with respect to $t$ or $z$ ) or interchange of summation and differentiation is assumed to be valid formally. A rigorous validation requires the proof of uniform convergency with a radius of convergence greater than unity.

${ }^{4}$ Throughout this thesis, the accent $\hat{x}$ is used to indicate the description of $x$ by a formal power series.
} 
The coefficients $\hat{x}_{n}(t)$ of (2.23) can be determined from the PDE (2.1) with $\Psi(x)=0$ and BCs (2.3), (2.4) by formal differentiation of $\hat{x}(z, t)$ with respect to $t$ and $z$

$$
\begin{aligned}
\frac{\partial \hat{x}(z, t)}{\partial t} & =\sum_{n=0}^{\infty} \dot{\hat{x}}_{n}(t) z^{n} \\
\frac{\partial^{i} \hat{x}(z, t)}{\partial z^{i}} & =\sum_{n=0}^{\infty}\left[\prod_{j=1}^{i}(n+j)\right] \hat{x}_{n+i}(t) z^{n}, \quad i \in \mathbb{N} .
\end{aligned}
$$

Substitution into (2.1) and sorting terms of equal order in $z^{n}$ yields

$$
\sum_{n=0}^{\infty} z^{n}\left[\dot{\hat{x}}_{n}(t)-\lambda(n+2)(n+1) \hat{x}_{n+2}(t)-\nu(n+1) \hat{x}_{n+1}(t)-\beta \hat{x}_{n}(t)\right]=0 .
$$

Since this expression has to hold for any $z \in(0,1)$, it follows that

$$
\hat{x}_{n+2}(t)=\frac{\dot{\hat{x}}_{n}(t)-\nu(n+1) \hat{x}_{n+1}(t)-\beta \hat{x}_{n}(t)}{\lambda(n+2)(n+1)}, \quad n \in \mathbb{N}_{0} .
$$

On the other hand, formal evaluation of the BCs (2.3), (2.4) results in

$$
\begin{aligned}
\hat{x}_{1}(t) & =0 \\
\hat{u}(t) & =\sum_{n=0}^{\infty}\left(p_{1}(n+1) \hat{x}_{n+1}(t)+r_{1} \hat{x}_{n}(t)\right) .
\end{aligned}
$$

In view of (2.24), a unique determination of the series coefficients by sequential evaluation requires a specification of two starting conditions, namely $\hat{x}_{0}(t)$ and $\hat{x}_{1}(t)$. The value of $\hat{x}_{1}(t)$ follows directly from (2.25). Since no further information on the coefficients can be extracted from (2.26), consider the power series ansatz evaluated at $z=0$, i.e. $\hat{x}(0, t)=\hat{x}_{0}(t)$. If uniform convergence of the power series $\hat{x}(z, t)$ with a radius of convergence greater than unity is ensured such that $x(z, t) \equiv \hat{x}(z, t)$, the coefficient $\hat{x}_{0}(t)$ corresponds to the state value $x(0, t)$ at the boundary $z=0$, which will be denoted as $y(t)$, i.e

$$
\hat{x}_{0}(t)=y(t) .
$$

As a result, (2.24) can be interpreted as a differential recursion for the coefficients $\hat{x}_{n+2}(t)$ with starting conditions $\hat{x}_{0}(t)=y(t)$ and $\hat{x}_{1}(t)=0$. By induction it is possible to verify that the coefficients

$$
\begin{aligned}
\hat{x}_{0}(t) & =y(t) \\
\hat{x}_{1}(t) & =0 \\
\hat{x}_{2 n}(t) & =\frac{1}{(2 n) ! \lambda^{n}} \sum_{j=1}^{n} \varpi_{2 n, j}\left(\frac{\nu^{2}}{\lambda}\right)^{n-j} D^{j}\{y(t)\}, \quad n \geq 1 \\
\hat{x}_{2 n+1}(t) & =-\frac{\nu}{(2 n+1) ! \lambda^{n+1}} \sum_{j=1}^{n} \varpi_{2 n+1, j}\left(\frac{\nu^{2}}{\lambda}\right)^{n-j} D^{j}\{y(t)\}, \quad n \geq 1
\end{aligned}
$$


describe the unique solution to the differential recursion with $\varpi_{i, j}=\varpi_{i-1, j}+\varpi_{i-2, j-1}$, $\varpi_{i, 1}=$ $1, \varpi_{2 i, i}=1$ for $i \geq 1, j \geq 2$, and operator $D=d / d t-\beta, D^{j}=D\left\{D^{j-1}\right\}, j \geq 1$.

As for the linear heat equation, the proof of convergency obviously refers to a problem of motion planning by choosing functions $y(t)$ of a certain Gevrey order:

Theorem 2.2. The series (2.23) with coefficients (2.28) converges to the solution of (2.1)(2.4) for all $z$ if $y(t)$ is Gevrey of order $\alpha \leq 2$.

The explicit proof can be performed similarly to the proof of Theorem 2.1. Alternatively Lynch and Rudolph (2002) provide an approach to verify uniform convergence which directly acts on the differential recursion and is hence applicable to more general cases where the parameterization is not available in closed form. ${ }^{5}$ Thus the proof of Theorem 2.2 reads as follows:

Proof. By induction, it can be concluded that the series coefficients $\hat{x}_{n}(t), n \geq 0$ satisfy

$$
\sup _{t \in \mathbb{R}^{+}}\left|\hat{x}_{n}^{(l)}(t)\right| \leq \frac{M \epsilon^{n}}{R^{l}} \frac{(n+l) !^{\alpha}}{(n !)^{\frac{\alpha}{2}+1}},
$$

such that application of the Cauchy-Hadamard Theorem A.1 yields the radius of convergence $\rho$ of the series $\hat{x}(z, t)=\sum_{n} \hat{x}_{n}(t) z^{n}$ as

$$
\rho= \begin{cases}\infty & \text { for } \alpha<2 \\ \frac{1}{\epsilon} & \text { for } \alpha=2 \\ 0 & \text { for } \alpha>2\end{cases}
$$

For $n=0$, Eqn. (2.29) directly holds since $y(t)$ is assumed to be Gevrey of order $\alpha$. This carries over to $n=1$ since $\hat{x}_{1}(t)=0$ by definition. Shifting indices and differentiating the differential recursion (2.24) provides

$$
\left|\hat{x}_{n}^{(l)}\right| \leq \frac{1}{n(n-1) \lambda}\left(\left|\hat{x}_{n-2}^{(l+1)}\right|+\nu(n-1)\left|\hat{x}_{n-1}^{(l)}\right|+|\beta|\left|\hat{x}_{n-2}^{(l)}\right|\right), \quad n \geq 2 .
$$

With (2.29), it follows that

$$
\begin{aligned}
\sup _{t \in \mathbb{R}^{+}}\left|\hat{x}_{n}^{(l)}(t)\right| & \leq \frac{M \epsilon^{n}}{R^{l}} \frac{(n+l) !^{\alpha}}{(n !)^{\frac{\alpha}{2}+1}}\left(\frac{1}{\lambda \epsilon^{2} R} \frac{[n(n-1)]^{\frac{\alpha}{2}}}{(n+l)^{\alpha}}+\frac{\nu}{\lambda \epsilon} \frac{n^{\frac{\alpha}{2}}}{(n+l)^{\alpha}}+\frac{|\beta|}{\lambda \epsilon^{2}} \frac{[n(n-1)]^{\frac{\alpha}{2}}}{[(n+l)(n+l-1)]^{\alpha}}\right) \\
& \leq \frac{M \epsilon^{n}}{R^{l}} \frac{(n+l) !^{\alpha}}{(n !)^{\frac{\alpha}{2}+1}} \underbrace{\left(\frac{1}{\lambda \epsilon^{2} R}+\frac{\nu}{\sqrt{2} \lambda \epsilon}+\frac{|\beta|}{\lambda \epsilon^{2}}\right)}_{h(\epsilon)} .
\end{aligned}
$$

Setting the latter expression $h(\epsilon)=1$ yields a quadratic equation for $\epsilon$, whose greater root determines $\epsilon$ in (2.29). As a result, the radius of convergence of the series (2.23) with coefficients (2.24), (2.25), (2.27) is given by (2.30). Hence uniform convergency with infinite radius of convergence of the series parameterization is ensured if $\alpha<2$. A finite radius of convergence is obtained for $\alpha=2$.

\footnotetext{
${ }^{5}$ Note that due to the use of the formal power series ansatz (2.23), the approach of Lynch and Rudolph (2002) has to be slightly adopted.
} 
Since at least a unit radius of convergence is verified for functions $y(t)$ of Gevrey order $\alpha \leq 2$, the input parameterization (2.26) can be truncated at some integer $N$ for numerical evaluation

$$
u_{N}(t)=\sum_{n=0}^{N}\left(p_{1}(n+1) \hat{x}_{n+1}(t)+r_{1} \hat{x}_{n}(t)\right) .
$$

Thereby, for given $\epsilon>0$ there always exists an $N_{\epsilon} \in \mathbb{N}$ such that $\left|u(t)-u_{N}(t)\right| \leq \epsilon$ for $N \geq N_{\epsilon}$. Hence, a sufficiently accurate approximation of $u(t)$ or respectively $\hat{u}(t)$ can be obtained by considering $u_{N}(t)$.

Although convergency of the series solution is ensured by Theorem 2.2, the speed of convergence is unknown. In fact, depending on the strength of convection represented by the parameter $\nu$, the series (2.31) has to be truncated at different $N$ in order to provide a meaningful boundary input to track the desired trajectory. Simulation results depicted in Figure 2.2 for two values of $N \in\{20,25\}$ and varied convection parameter $\nu \in\{0,10,15,17,20,25\}$ illustrate this effect. In particular, a rapid change between smooth and oscillating behaviour of the feedforward term $u_{N}(t)$ can be observed (e.g. between $\nu=15$ and $\nu=20$ for $N=20$ ), whereby the transition point can be postponed to higher convection values if the number of series coefficients in increased.

\subsubsection{Scalar DCR-equations with polynomial nonlinearities}

In the following, the nonlinear parabolic DCRS (2.1)-(2.4) is studied using a formal power series approach. Therefore similar to the previous section, formally substitute the ansatz (2.23) into PDE (2.1) and BCs (2.3), (2.4). Assuming polynomial nonlinearities, i.e.

$$
\Psi(x)=\sum_{j=0}^{J} \mu_{j} x^{2+j}, \quad J \geq 0
$$

then the following differential recursion can be derived using sequential application of the Cauchy product formula for powers of series

$$
\begin{aligned}
\hat{x}_{n+2}(t) & =\frac{\dot{\hat{x}}_{n}(t)-\nu(n+1) \hat{x}_{n+1}(t)-\beta \hat{x}_{n}(t)-\hat{\Psi}_{n}\left(\underline{\hat{x}}_{n}(t)\right)}{\lambda(n+2)(n+1)} \\
\hat{x}_{1}(t) & =0
\end{aligned}
$$

where the nonlinearities are summarized in

$$
\hat{\Psi}_{n}\left(\underline{\hat{x}}_{n}\right)=\sum_{j=0}^{J} \mu_{j} \tilde{\Psi}_{n}^{j+1}\left(\underline{\hat{x}}_{n}\right)
$$

with

$$
\tilde{\Psi}_{j_{i}}^{i}\left(\underline{\hat{x}}_{j_{i}}\right)=\sum_{j_{i-1}=0}^{j_{i}} \hat{x}_{j_{i}-j_{i-1}} \tilde{\Psi}_{j_{i-1}}^{i-1}\left(\underline{\hat{x}}_{j_{i-1}}\right), \quad i=2,3, \ldots, J-1, \quad \tilde{\Psi}_{j_{1}}^{1}=\sum_{j_{0}=0}^{j_{1}} \hat{x}_{j_{1}-j_{0}} \hat{x}_{j_{0}}
$$



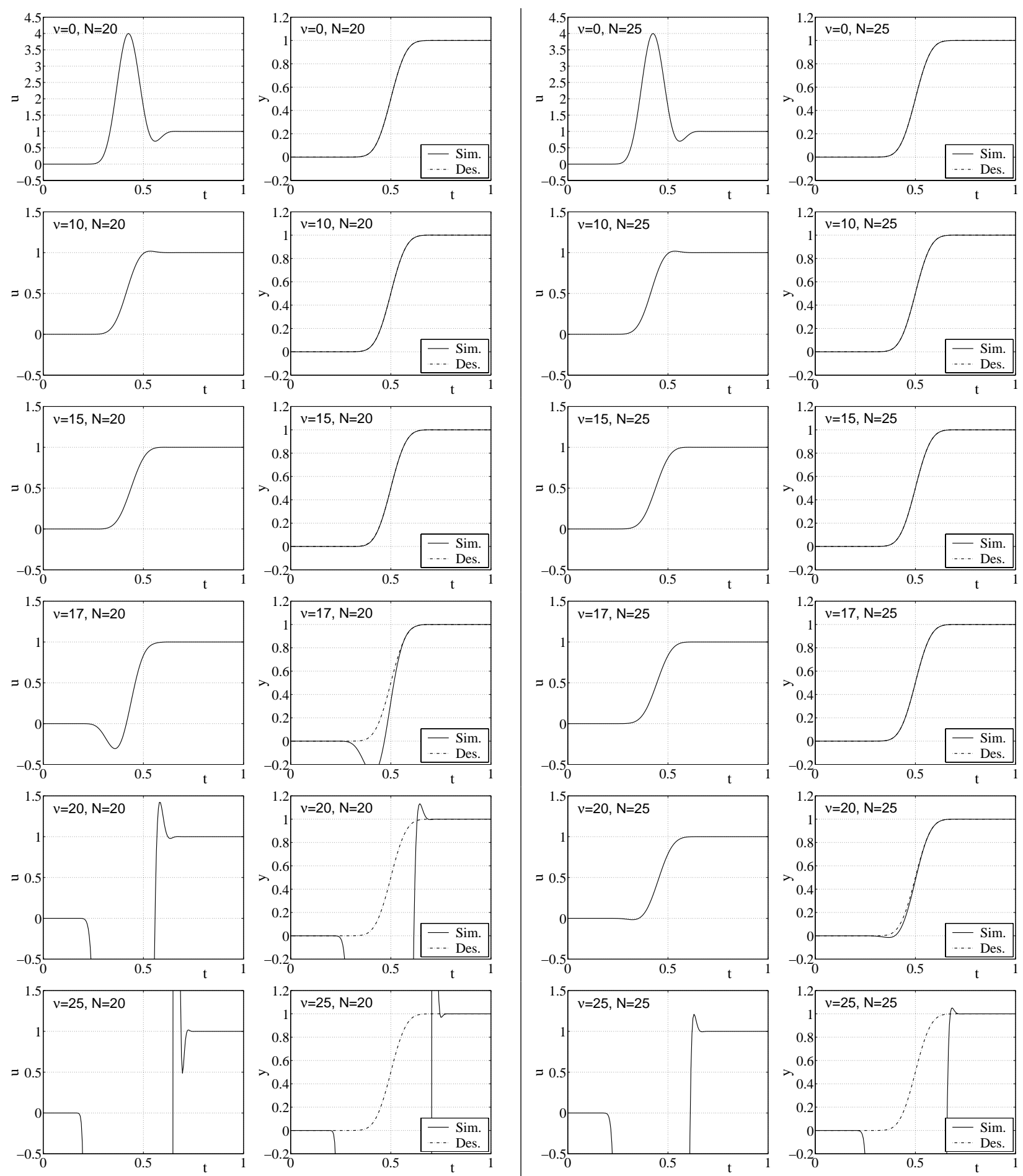

Figure 2.2: Comparison of numerical results for open-loop boundary control of the linear diffusionconvection system (2.1)-(2.4) with $\Psi(x)=0$ for desired trajectory $y_{d}(t)=\Phi_{\gamma, T}(t)$ from (2.17) with $\gamma=2, T=1$. Left: results for truncation at $N=20$ - feedforward control $u_{N}(t)$ from $(2.31)$ and output trajectory $y(t)=x(0, t)$ compared to desired trajectory $y_{d}(t)$; right: $N=25$. 
and $\underline{\hat{x}}_{n}(t)=\left[\hat{x}_{0}(t), \hat{x}_{1}(t), \ldots, \hat{x}_{n}(t)\right]^{T}$ for $n \in \mathbb{N}_{0}$. It can be easily verified that $\sum_{j_{i}=0}^{\infty} \tilde{\Psi}_{j_{i}}^{i}\left(\underline{\hat{x}}_{j_{i}}\right) z^{j_{i}}$ corresponds to the Cauchy product evaluation of $\hat{x}^{i+1}(z, t), i \geq 1$.

Since the differential recursion (2.33) is of second order, its unique solution requires two starting conditions. Besides (2.34), substitution of the free BC $y(t)=x(0, t)$ results in

$$
\hat{x}_{0}(t)=y(t) .
$$

The structure of the differential recursion (2.33) with starting conditions (2.34), (2.36) clearly illustrates, that a closed-form solution cannot be expected. Nevertheless, uniform convergence of the series has to be ensured in order to obtain a solution to the scalar DCRS (2.1)-(2.4) and to validate the performed operations such as differentiation with respect to $t$ and $z$ as well as the interchange of summation and multiplication.

The convergence condition for the special case $J=1$, i.e. quadratic and cubic terms of the state appear in the PDE, reads as follows:

Theorem 2.3. The series solution (2.23) with coefficients satisfying the differential recursion (2.33)-(2.36) converges to the solution of the DCRS (2.1)-(2.4) with at least a unit radius of convergence if $y(t)$ is Gevrey of order $\alpha \leq 2$ with constants $M, R$ and if the following inequality is satisfied

$$
\nu+\sqrt{\nu^{2}+16 \lambda\left[\frac{1}{R}+\frac{1}{2}\left(|\beta|+M\left|\mu_{0}\right|+M^{2}\left|\mu_{1}\right|\right)\right]} \leq 4 \lambda .
$$

The proof utilizes some ideas of (Lynch and Rudolph, 2002) and extends their results to the case of cubic nonlinearites (Meurer and Zeitz, 2004b).

Proof. By induction, it is shown that

$$
\sup _{t \in \mathbb{R}^{+}}\left|\hat{x}_{n}^{(l)}(t)\right| \leq \frac{M \epsilon^{n}}{R^{l}} \frac{(n+l) !^{\alpha}}{n !^{\alpha}} .
$$

Therefore shifting indices and taking successive derivatives of the differential recursion (2.33)(2.36) with $J=1$ yields for $n \geq 2$ and arbitrary $l \in \mathbb{N}_{0}$

$$
\lambda \hat{x}_{n}^{(l)}=\hat{x}_{n-2}^{(l+1)}-\nu \hat{x}_{n-1}^{(l)}-\beta \hat{x}_{n-2}^{(l)}-\sum_{i=0}^{n-2} \sum_{r=0}^{l}\left(\begin{array}{l}
l \\
r
\end{array}\right) \hat{x}_{n-2-i}^{(r)}\left[\mu_{0} \hat{x}_{i}^{(l-r)}+\mu_{1} \sum_{j=0}^{i} \sum_{q=0}^{l-r}\left(\begin{array}{c}
l-r \\
q
\end{array}\right) \hat{x}_{j}^{(l-r-q)} \hat{x}_{i-j}^{(q)}\right],
$$

with $\hat{x}_{0}^{(l)}(t)=y^{(l)}(t)$ and $\hat{x}_{1}^{(l)}(t)=0$. Substituting (2.38), it follows that

$$
\begin{aligned}
\lambda\left|\hat{x}_{n}^{(l)}\right| \leq & \frac{M \epsilon^{n-2}}{R^{l}}\left[\frac{(n-1+l) !^{\alpha}}{R(n-2) !^{\alpha}}+\frac{\nu}{\epsilon} \frac{(n-1+l) !^{\alpha}}{(n-1) !^{\alpha}}+|\beta| \frac{(n-2+l) !^{\alpha}}{(n-2) !^{\alpha}}+\right. \\
& \left|\mu_{0}\right| M \sum_{i=0}^{n-2} \frac{\left(\begin{array}{c}
n-2 \\
i
\end{array}\right)}{(i !(n-2-i) !)^{\alpha}} \times \sum_{r=0}^{l}\left(\begin{array}{l}
l \\
r
\end{array}\right)(i+l-r) !^{\alpha}(n-2-i+r) !^{\alpha}+ \\
& \left|\mu_{1}\right| M^{2} \sum_{i=0}^{n-2} \frac{\left(\begin{array}{c}
n-2 \\
i
\end{array}\right)}{(n-2-i) !^{\alpha}} \sum_{r=0}^{l}\left(\begin{array}{c}
l \\
r
\end{array}\right)(n-2-i+r) !^{\alpha} \times \\
& \left.\sum_{j=0}^{i} \frac{\left(\begin{array}{c}
i \\
j
\end{array}\right)}{(j !(i-j) !)^{\alpha}} \sum_{q=0}^{l-r}\left(\begin{array}{c}
l-r \\
q
\end{array}\right)(j+l-r-q) !^{\alpha}(i-j+q) !^{\alpha}\right] .
\end{aligned}
$$



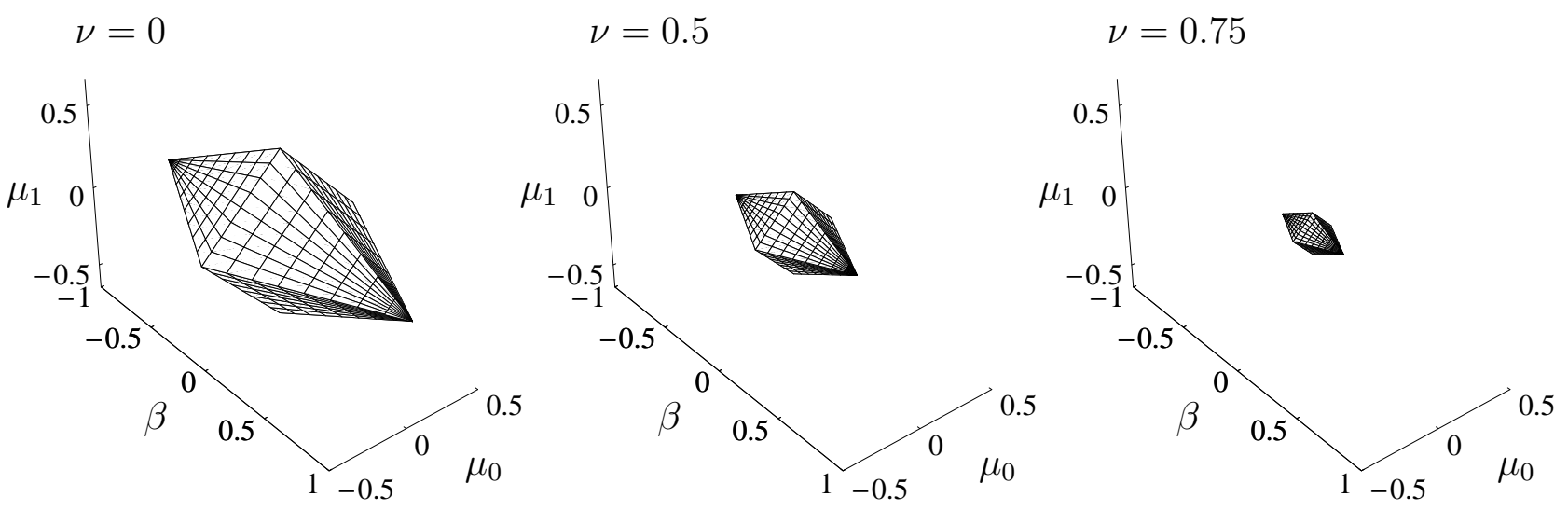

Figure 2.3: Regions of uniform convergence (inside body) for series solution (2.33)-(2.36) described by inequality (2.37) in parameter space $\left\{\beta, \mu_{0}, \mu_{1}\right\}$ for fixed $\lambda=1, M=1, R=2$ and $\nu \in\{0,0.5,0.75\}$ varied.

Successive application of Lemmas A.2 and A.3 of the Appendix A yields $\lambda\left|\hat{x}_{n}^{(l)}\right| \leq \frac{M \epsilon^{n}(n+l) !^{\alpha}}{R^{l} n !^{\alpha}}\left[\frac{(n(n-1))^{\alpha-1}}{\epsilon^{2} R(n+l)^{\alpha}}+\frac{\nu n^{\alpha-1}}{\epsilon(n+l)^{\alpha}}+\frac{|\beta|(n(n-1))^{\alpha-1}}{\epsilon^{2}((n+l)(n+l-1))^{\alpha}}+\frac{\left|\mu_{0}\right| M}{\epsilon^{2} n}+\frac{\left|\mu_{1}\right| M^{2}}{2 \epsilon^{2}}\right]$.

Since $n \geq 2, l \geq 0,1 \leq \alpha \leq 2$ an upper bound independent of $n$ and $l$ can be derived

$$
\left|\hat{x}_{n}^{(l)}\right| \leq \frac{M \epsilon^{n}(n+l) !^{\alpha}}{\lambda R^{l} n !^{\alpha}} \underbrace{\left[\frac{1}{R \epsilon^{2}}+\frac{\nu \epsilon+|\beta|+\left|\mu_{0}\right| M+\left|\mu_{1}\right| M^{2}}{2 \epsilon^{2}}\right]}_{=: h(\epsilon)} .
$$

Using the Cauchy-Hadamard formula for $\alpha \in[1,2]$, the radius of convergence $\rho$ of the formal power series $\sum_{n} \hat{x}_{n}(t) z^{n}$ with the above bound is given by $\rho=\frac{1}{\epsilon}$. Hence solving $h(\epsilon)=\lambda$ for $\epsilon$ and taking the maximal $\epsilon$ yields inequality (2.37) which completes the proof.

As can be clearly deduced from inequality (2.37), the sets of admissible parameters $\mathcal{P}=$ $\left\{\lambda, \nu, \beta, \nu_{0}, \nu_{1}\right\}$ and trajectories represented by $\{M, R\}$ are rather limited and usually outside a practically relevant range as shown in Figure 2.3: for increasing $\nu$ the region of convergence contracts to a point and vanishes afterwards. In particular, it is necessary to require $R>1$ to satisfy (2.37) for given $M$ and parameter sets $\mathcal{P}$. Furthermore, the diffusion parameter $\lambda$ must be large compared to the convection parameter $\nu$, i.e. only diffusion dominated systems can be considered within this framework. Prior to presenting an approach in Chapters 3 and 4 to bypass these limitations, the ideas of Section 2.1 are generalized to boundary controlled systems of DCR-equations.

\subsection{MIMO DCR-equations with boundary inputs}

The promising results of Section 2.1 clearly illustrate the applicability of formal power series to control design for scalar DCR-systems, although certain restrictions remain. On the other 
hand, typical real-world problems consist of systems of parabolic nonlinear DCR-equations originating e.g. from chemical or biochemical engineering. Important examples include tubular fixed-bed reactor for production or tubular bioreactors for degradation processes. Therefore in this chapter, the formal power series approach is generalized to certain systems of boundary controlled nonlinear DCR-equations, with the focus on systematic feedforward tracking control design. General results are derived by formally analyzing a generic DCR model description. In the second part, these results are applied to formally parameterize a non-isothermal tubular reactor model with boundary control.

\subsubsection{General DCR-equations}

In the following, the formal power series parameterization of states and inputs is considered for a general system of $K$ second-order parabolic PDEs with $M$ input functions on $(z, t) \in(0,1) \times \mathbb{R}^{+}$, i.e.

$$
\frac{\partial \boldsymbol{x}(z, t)}{\partial t}=S \frac{\partial^{2} \boldsymbol{x}(z, t)}{\partial z^{2}}+\boldsymbol{\Psi}\left(\boldsymbol{x}(z, t), \frac{\partial \boldsymbol{x}(z, t)}{\partial z}\right), \quad z \in(0,1), t>0
$$

with initial conditions (IC)

$$
\boldsymbol{x}(z, 0)=\boldsymbol{x}_{0}(z), \quad z \in[0,1]
$$

and boundary conditions $(\mathrm{BC})$

$$
\begin{array}{ll}
P_{0} \frac{\partial \boldsymbol{x}}{\partial z}(0, t)+R_{0} \boldsymbol{x}(0, t) & =\boldsymbol{\zeta}(t), \quad t>0 \\
P_{1} \frac{\partial \boldsymbol{x}}{\partial z}(1, t)+R_{1} \boldsymbol{x}(1, t) & =Q \boldsymbol{u}(t), \quad t>0 .
\end{array}
$$

Thereby it is assumed, that state $\boldsymbol{x}(z, \cdot)$ and input $\boldsymbol{u}(\cdot)$ evolve on suitable functional spaces $\mathcal{X}[0,1]$ and $\mathcal{U}$, respectively. The smooth $(2 K-M) \times 1$ vector $\boldsymbol{\zeta}(t) \in \mathcal{C}^{\infty}$ on the righthand side of (2.41) is assumed to summarize known functions of time to represent possible inhomogeneous BCs. Furthermore, it is assumed that the matrix $S \in \mathbb{R}^{K \times K}$ is of diagonal form $^{6}$ and the input matrix $Q \in \mathbb{R}^{M \times M}$ is assumed non-singular. Since spatial differentiation in (2.39) is limited to second order, it follows that the matrices in the boundary conditions satisfy $P_{0}, R_{0} \in \mathbb{R}^{(2 K-M) \times K}$ while $P_{1}, R_{1} \in \mathbb{R}^{M \times K}$.

Remark 2.1. The following considerations are not limited to the case where $K=M$, i.e. the number of states equals the number of inputs, which provides an extension of the results available in respective literature on the power series analysis of boundary controlled PDEs.

\footnotetext{
${ }^{6}$ This can be achieved for any given non-singular matrix $Q \in \mathbb{R}^{K \times K}$ by modal decomposition, i.e. $Q=$ $V S V^{-1}$, where $V=\left[\boldsymbol{v}_{1}, \ldots, \boldsymbol{v}_{K}\right], S=\operatorname{Diag}\left\{\lambda_{1}, \ldots, \lambda_{K}\right\}$ with $\boldsymbol{v}_{i}$ being the $i^{\text {th }}$ eigenvector corresponding the $i^{\text {th }}$ eigenvalue $\lambda_{i}$. Hence introduction of the new states $\boldsymbol{x}(z, t)=V \boldsymbol{x}^{\prime}(z, t)$ provides a PDE structure consistent with the assumed one (2.39). Note that such a transformation always exists, since the system is assumed to be parabolic.
} 
For the application of the parameterization approach, it is assumed that there exists a unique formal power series solution $\hat{\boldsymbol{x}}(z, t)$ in the spatial coordinate with coefficients $\hat{\boldsymbol{x}}_{n}(t), n \in \mathbb{N}_{0}$ from a suitable Banach space, i.e.

$$
\hat{\boldsymbol{x}}(z, t)=\sum_{n=0}^{\infty} \hat{\boldsymbol{x}}_{n}(t) z^{n}
$$

such that $\hat{\boldsymbol{x}}(z, t)$ represents the series expansion of $\boldsymbol{x}(z, t)$. Hence, the following operations are only applied formally, since they require uniform convergence of the formal power series $\hat{\boldsymbol{x}}(z, t)$. Substitution of (2.43) into the PDEs (2.39) yields

$$
\sum_{n=0}^{\infty}\left[\frac{d \hat{\boldsymbol{x}}_{n}(t)}{d t}-(n+2)(n+1) S \hat{\boldsymbol{x}}_{n+2}(t)\right] z^{n}=\boldsymbol{\Psi}\left(\sum_{n=0}^{\infty} \hat{\boldsymbol{x}}_{n}(t) z^{n}, \sum_{n=0}^{\infty}(n+1) \hat{\boldsymbol{x}}_{n+1}(t) z^{n}\right) .
$$

In view of this equation, terms of equal order in $z$ can be sorted if the nonlinear source term $\boldsymbol{\Psi}(\boldsymbol{x}, \partial \boldsymbol{x}, / \partial z)$ is restricted to functions satisfying the following assumption:

Assumption 2.1. ${ }^{7}$ The nonlinear source $\boldsymbol{\Psi}\left(\boldsymbol{x}(z, t), \frac{\partial \boldsymbol{x}(z, t)}{\partial z}\right)$ in PDE (2.39) formally allows a power series expansion with respect to (2.43) of the form

$$
\left.\boldsymbol{\Psi}\left(\boldsymbol{x}(z, t), \frac{\partial \boldsymbol{x}(z, t)}{\partial z}\right)\right|_{\boldsymbol{x}(z, t) \rightarrow \hat{\boldsymbol{x}}(z, t)}=\sum_{n=0}^{\infty} \hat{\boldsymbol{\Psi}}_{n}\left(\underline{\hat{\boldsymbol{x}}}_{n+1}(t)\right) z^{n}
$$

where

$$
\underline{\hat{\boldsymbol{x}}}_{n+1}(t):=\left[\hat{\boldsymbol{x}}_{0}^{T}(t), \hat{\boldsymbol{x}}_{1}^{T}(t), \hat{\boldsymbol{x}}_{2}^{T}(t), \ldots, \hat{\boldsymbol{x}}_{n+1}^{T}(t)\right]^{T}
$$

and the time-variable coefficients $\hat{\mathbf{\Psi}}_{n}\left(\underline{\hat{\boldsymbol{x}}}_{n+1}(t)\right)=\left[\hat{\Psi}_{1}\left(\underline{\hat{\boldsymbol{x}}}_{n+1}(t)\right), \ldots, \hat{\Psi}_{K}\left(\underline{\hat{\boldsymbol{x}}}_{n+1}(t)\right)\right]^{T}$ being smooth with respect to their arguments, i.e.

$$
\forall i \in \mathbb{N} \wedge \forall n \in \mathbb{N}_{0}: \quad \exists \frac{\partial^{i} \hat{\Psi}_{j, n}\left(\underline{\hat{x}}_{n+1}\right)}{\partial \hat{x}_{l, m}^{i}} \quad \text { with } l \in\{1,2, \ldots, K\}, m \in 0,1, \ldots, n+1 .
$$

Since the PDE (2.39) is defined for all $z \in(0,1)$, the second order differential recursion

$$
\hat{\boldsymbol{x}}_{n+2}(t)=\frac{S^{-1}}{(n+2)(n+1)}\left(\dot{\hat{\boldsymbol{x}}}_{n}(t)-\hat{\mathbf{\Psi}}_{n}\left(\underline{\hat{\boldsymbol{x}}}_{n+1}(t)\right)\right), \quad \forall n \in \mathbb{N}_{0}
$$

can be derived from (2.44)-(2.45) for the series coefficients $\hat{\boldsymbol{x}}_{n}(t), n \geq 2$. For a unique solution, two starting conditions for the coefficients $\hat{\boldsymbol{x}}_{0}(t)$ and $\hat{\boldsymbol{x}}_{1}(t)$ have to be specified. Therefore, formally evaluate BC (2.41) with the power series ansatz (2.43), i.e.

$$
P_{0} \hat{\boldsymbol{x}}_{1}(t)+R_{0} \hat{\boldsymbol{x}}_{0}(t)=\boldsymbol{\zeta}(t) .
$$

\footnotetext{
${ }^{7}$ Note that the imposed restriction is rather weak, since it is only required that $\boldsymbol{\Psi}\left(\boldsymbol{x}(z, t), \frac{\partial \boldsymbol{x}(z, t)}{\partial z}\right)$ can be either expanded in a convergent Taylor series or approximated by a polynomial in $\boldsymbol{x}(z, t)$ and $\frac{\partial \boldsymbol{x}(z, t)}{\partial z}$ of finite degree. For the latter case, Eqn. (2.45) directly follows from the application of Cauchy's product formula.
} 
Note that no further information on the series coefficients $\hat{\boldsymbol{x}}_{0}(t)$ and $\hat{\boldsymbol{x}}_{1}(t)$ can be obtained from $\mathrm{BC}(2.42)$, since

$$
\hat{\boldsymbol{u}}(t)=Q^{-1} \sum_{n=0}^{\infty}\left[R_{1} \hat{\boldsymbol{x}}_{n}(t)+(n+1) P_{1} \hat{\boldsymbol{x}}_{n+1}(t)\right] .
$$

Hence instead of considering (2.42), additional imposed BCs are specified

$$
F_{d} \frac{\partial \boldsymbol{x}}{\partial z}(0, t)+F_{v} \boldsymbol{x}(0, t)=\boldsymbol{y}(t)
$$

with yet unknown matrices $F_{d}, F_{v} \in \mathbb{R}^{M \times K}$. These equations introduce the (so far) fictious quantities $\boldsymbol{y}(t)$ in order to allow a parameterization of the states $\hat{\boldsymbol{x}}_{n}(t)$. In order to illustrate this, evaluate the natural BCs (2.41) and the imposed BCs (2.50) together with the formal power series ansatz (2.43), which yields a $2 K$-dimensional set of linear equations for the $2 K$ unknowns $\hat{\boldsymbol{x}}_{0}(t)$ and $\hat{\boldsymbol{x}}_{1}(t)$

$$
\underbrace{\left(\begin{array}{ll}
R_{0} & P_{0} \\
F_{v} & F_{d}
\end{array}\right)}_{=: \Theta}\left[\begin{array}{l}
\hat{\boldsymbol{x}}_{0}(t) \\
\hat{\boldsymbol{x}}_{1}(t)
\end{array}\right]=\left[\begin{array}{l}
\boldsymbol{\zeta}(t) \\
\boldsymbol{y}(t)
\end{array}\right] .
$$

If the matrix $\Theta$ is non-singular, i.e. $\exists \Theta^{-1}=\left[\Theta_{1,1}^{-1}, \Theta_{1,2}^{-1} \mid \Theta_{2,1}^{-1}, \Theta_{2,2}^{-1}\right]$, the coefficients $\hat{\boldsymbol{x}}_{0}(t)$ and $\hat{\boldsymbol{x}}_{1}(t)$ can be uniquely determined

$$
\begin{aligned}
& \hat{\boldsymbol{x}}_{0}(t)=\Theta_{1,1}^{-1} \boldsymbol{\zeta}(t)+\Theta_{1,2}^{-1} \boldsymbol{y}(t)=: \boldsymbol{\Upsilon}_{0}(\boldsymbol{y}(t), \boldsymbol{\zeta}(t)) \\
& \hat{\boldsymbol{x}}_{1}(t)=\Theta_{2,1}^{-1} \boldsymbol{\zeta}(t)+\Theta_{2,2}^{-1} \boldsymbol{y}(t)=: \boldsymbol{\Upsilon}_{1}(\boldsymbol{y}(t), \boldsymbol{\zeta}(t))
\end{aligned}
$$

As a result, the differential recursion defined by (2.47) is uniquely solvable due to the starting conditions (2.52), (2.53). The solution, i.e. the series coefficients $\hat{\boldsymbol{x}}_{n}(t), n \geq 2$, can be determined by sequential evaluation of the recursion in terms of $\boldsymbol{y}(t)$ and its time-derivatives $\boldsymbol{y}^{(j)}(t), j \geq 1$ up to infinite order since $n \in \mathbb{N}_{0}$. This yields a schematic parameterization of the series coefficients in the following recursive representation

$$
\begin{aligned}
& \hat{\boldsymbol{x}}_{2 n}(t)=\frac{S^{-1}}{(2 n)(2 n-1)}\left(\frac{\partial \boldsymbol{\Upsilon}_{2 n-2}\left(\underline{\boldsymbol{y}}_{n-1}, \underline{\boldsymbol{\zeta}}_{n-1}\right)}{\partial \underline{\boldsymbol{y}}_{n-1}} \underline{\boldsymbol{y}}_{n-1}+\frac{\partial \boldsymbol{\Upsilon}_{2 n-2}\left(\underline{\boldsymbol{y}}_{n-1}, \underline{\boldsymbol{\zeta}}_{n-1}\right)}{\partial \underline{\boldsymbol{\zeta}}_{n-1}} \dot{\boldsymbol{\zeta}}_{n-1}\right. \\
& \left.-\hat{\boldsymbol{\Psi}}_{2 n-2}\left(\boldsymbol{\Upsilon}_{2 n-1}\left(\underline{\boldsymbol{y}}_{n-1}, \underline{\boldsymbol{\zeta}}_{n-1}\right)\right)\right)=: \boldsymbol{\Upsilon}_{2 n}\left(\underline{\boldsymbol{y}}_{n}(t), \underline{\boldsymbol{\zeta}}_{n}(t)\right)
\end{aligned}
$$

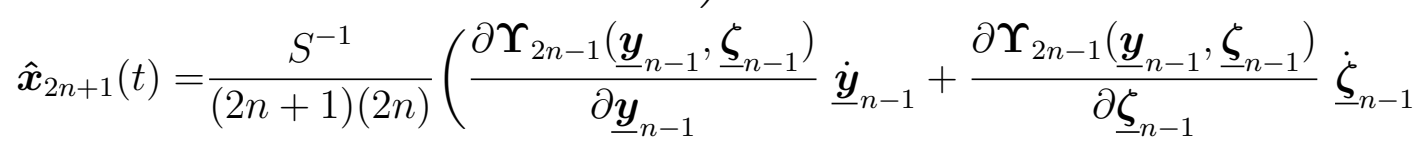

$$
\begin{aligned}
& \left.-\hat{\boldsymbol{\Psi}}_{2 n-1}\left(\boldsymbol{\Upsilon}_{2 n}\left(\underline{\boldsymbol{y}}_{n}, \underline{\boldsymbol{\zeta}}_{n}\right)\right)\right)=: \boldsymbol{\Upsilon}_{2 n+1}\left(\underline{\boldsymbol{y}}_{n}(t), \underline{\boldsymbol{\zeta}}_{n}(t)\right),
\end{aligned}
$$

where

$$
\underline{\boldsymbol{y}}_{n}(t):=\left[\boldsymbol{y}^{T}(t), \dot{\boldsymbol{y}}^{T}(t), \ldots, \boldsymbol{y}^{(n) T}(t)\right]^{T}, \quad \underline{\boldsymbol{\zeta}}_{n}(t):=\left[\boldsymbol{\zeta}^{T}(t), \dot{\boldsymbol{\zeta}}^{T}(t), \ldots, \boldsymbol{\zeta}^{(n) T}(t)\right]^{T} .
$$


Note that depending on the choice of the matrices $F_{v}, F_{d}$ of the imposed BCs (2.50) to ensure non-singularity of $\Theta$, the dependency of the parameterizations (2.54), (2.55) on $\boldsymbol{y}(t)$ and its derivatives might be different with the latter equations representing the most general case (see e.g. tubular reactor example in the next section).

Hence substitution of (2.54), (2.55) into the ansatz (2.43) formally provides the power series expansion of $\boldsymbol{x}(z, t)$ in terms of $\boldsymbol{y}(t)$, i.e.

$$
\hat{\boldsymbol{x}}(z, t)=\sum_{n=0}^{\infty}\left[\boldsymbol{\Upsilon}_{2 n}\left(\underline{\boldsymbol{y}}_{n}(t), \underline{\boldsymbol{\zeta}}_{n}(t)\right) z^{2 n}+\boldsymbol{\Upsilon}_{2 n+1}\left(\underline{\boldsymbol{y}}_{n}(t), \underline{\boldsymbol{\zeta}}_{n}(t)\right) z^{2 n+1}\right] .
$$

Furthermore, evaluation of (2.42) provides the formal parameterization of the boundary inputs

$$
\hat{\boldsymbol{u}}(t)=Q^{-1} \sum_{n=0}^{\infty}\left(\left[R_{1}+2 n P_{1}\right] \boldsymbol{\Upsilon}_{2 n}\left(\underline{\boldsymbol{y}}_{n}(t), \underline{\boldsymbol{\zeta}}_{n}(t)\right)+\left[R_{1}+(2 n+1) P_{1}\right] \boldsymbol{\Upsilon}_{2 n+1}\left(\underline{\boldsymbol{y}}_{n}(t), \underline{\boldsymbol{\zeta}}_{n}(t)\right)\right) .
$$

This expression can be exploited for feedforward tracking control design by assigning trajectories $t \mapsto \boldsymbol{y}_{d}(t)$ for $\boldsymbol{y}(t)$. Thereby convergence of the formal solution (2.57) must be ensured by an appropriate choice of $\boldsymbol{y}_{d}(t)$. As can be expected form the convergence results for scalar DCR-equations with polynomial nonlinearity in Section 2.1.3, the proof of convergency for the considered DPS-system is obviously more challenging and is likely to be accessible only for special cases as is illustrated for the model of a non-isothermal tubular reactor in the next section.

Summarizing the above results, a formal power series parameterization of the DPS (2.39)-(2.42) is available if the following Theorem holds:

Theorem 2.4. The diffusion-convection-reaction equation (2.39)-(2.42) is formal power series parameterizable, if the matrices $F_{d}$ and $F_{v}$ of the imposed $B C s$

$$
\boldsymbol{y}(t)=F_{d} \frac{\partial \boldsymbol{x}}{\partial z}(0, t)+F_{v} \boldsymbol{x}(0, t), \quad \operatorname{dim} \boldsymbol{y}=\operatorname{dim} \boldsymbol{u}
$$

are chosen such that the $(2 K \times 2 K)-$ matrix

$$
\Theta:=\left(\begin{array}{cc}
R_{0} & P_{0} \\
F_{v} & F_{d}
\end{array}\right)
$$

with $R_{0}, P_{0}$ from (2.41) is non-singular.

Proof. See the derivation above.

This Theorem directly provides a simple test, whether a formal power series parameterization is possible and in particular yields a generalization and systematization of the more heuristical approach of Section 2.1 to introduce a parameterizing function. Nevertheless as pointed out before, the main restrictions are imposed by the necessary verification of convergence. For the following case of a non-isothermal tubular reactor boundary control problem, these conditions can be determined and motivate the introduction of a more powerful approach to recover the original states $\boldsymbol{x}(z, t)$ from the parameterized expansion $\hat{\boldsymbol{x}}(z, t)$ given by $(2.57)$. 


\subsubsection{A tubular reactor model}

As a generic example, the pseudohomogeneous axial dispersion model of a tubular reactor in case of an irreversible first order liquid phase reaction $A \rightarrow B$ (Ray, 1981; Jensen and Ray, 1982) as depicted in Figure 2.4 is considered. The PDEs for the tubular reactor in dimensionless

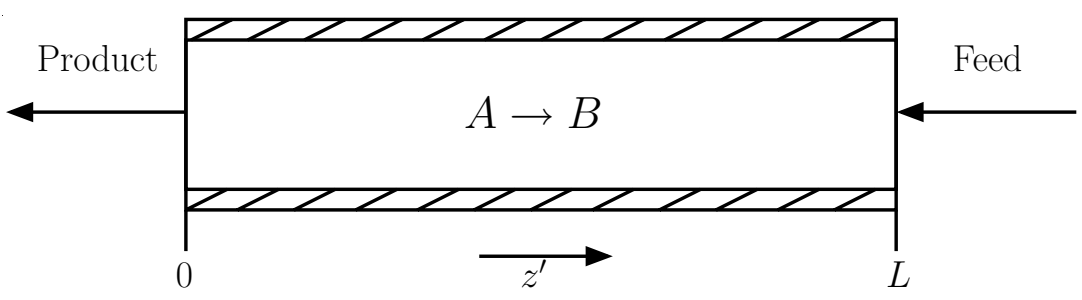

Figure 2.4: Schematic of a tubular reactor with first order liquid phase reaction $A \rightarrow B$ and spatial coordinate $z^{\prime} \in[0, L]$.

form (Ray, 1981; Jensen and Ray, 1982) read as

$$
\frac{\partial \boldsymbol{x}}{\partial t}=S \frac{\partial^{2} \boldsymbol{x}}{\partial z^{2}}+\boldsymbol{\Psi}\left(\boldsymbol{x}, \frac{\partial \boldsymbol{x}}{\partial z}\right), \quad z \in(0,1), t>0
$$

for the states $\boldsymbol{x}(z, t):=\left[x_{1}(z, t), x_{2}(z, t)\right]^{T}$ with boundary

$$
\begin{array}{ll}
\frac{\partial \boldsymbol{x}}{\partial z}(0, t)=\mathbf{0}, & t>0 \\
P_{1} \frac{\partial \boldsymbol{x}}{\partial z}(1, t)+\boldsymbol{x}(1, t)=\boldsymbol{u}(t), & t>0
\end{array}
$$

and consistent initial conditions

$$
\boldsymbol{x}(z, 0)=\boldsymbol{x}_{0}(z), \quad z \in[0,1]
$$

In the PDEs (2.61), the diffusion matrix is given by

$$
S=\left(\begin{array}{cc}
\frac{L e}{P e_{1}} & 0 \\
0 & \frac{1}{P e_{2}}
\end{array}\right)
$$

and convective as well as reactive effects are summarized in the vector

$$
\boldsymbol{\Psi}\left(\boldsymbol{x}(z, t), \frac{\partial \boldsymbol{x}(z, t)}{\partial z}\right)=\left[\begin{array}{c}
L e \frac{\partial x_{1}}{\partial z}+\operatorname{LeDa}\left(1-x_{1}\right) \exp \left(\frac{x_{2}}{1+x_{2} / \sigma}\right) \\
\frac{\partial x_{2}}{\partial z}-\beta\left(x_{2}-x_{2 w}\right)+B D a\left(1-x_{1}\right) \exp \left(\frac{x_{2}}{1+x_{2} / \sigma}\right)
\end{array}\right] .
$$

The matrix $P_{1}$ in the Danckwert's boundary conditions $(2.62),(2.63)$ takes the form

$$
P_{1}=\left(\begin{array}{cc}
\frac{1}{P e_{1}} & 0 \\
0 & \frac{1}{P e_{2}}
\end{array}\right)
$$


Here, $P e_{1}$ and $P e_{2}$ denote the Peclet numbers for mass and heat transfer, respectively, Le the Lewis number, $D a$ the Damköhler number, $B$ the adiabatic temperature rise, and $\beta, \gamma$ represent characteristic quantities for heat exchange and reaction (Jensen and Ray, 1982). The dimensionless state $\boldsymbol{x}(z, t)$ stems from a normalization by $x_{1}(z, t)=\left(c_{0}-c(z, t)\right) / c_{0}$ and $x_{2}(z, t)=\left(T(z, t)-T_{0}\right) E_{A} /\left(R T_{0}^{2}\right)$ with some constant concentration $c_{0}$ and temperature $T_{0}$, e.g. a constant stationary profile, saturation concentration or temperature maximum. The boundary input $\boldsymbol{u}(t)$ in (2.63) corresponds to similarly normalized inlet concentration $u_{1}(t)$ and temperature $u_{2}(t)$. The parameters $E_{A}$ and $R$ represent the activation energy and general gas constant, respectively. The value $x_{2 w}=\left(T_{w}-T_{0}\right) E_{A} /\left(R T_{0}^{2}\right)$ denotes the normalized wall temperature, where $x_{2 w}=0$ if $T_{0}=T_{w}$ with constant $T_{w}$. Furthermore, temporal and spatial coordinates are normalized according to $t=t^{\prime} v_{z} /\left(\right.$ Le $\left._{p} L\right)$ and $z=z^{\prime} / L$ with gas velocity $v_{z}$, bed porosity $\epsilon_{p}$, and reactor length $L$.

The control problem concerns the design of the boundary inputs $\boldsymbol{u}(t)=\left[u_{1}(t), u_{2}(t)\right]^{T}$ in order to track the output

$$
\boldsymbol{w}(t)=\boldsymbol{x}(0, t) \rightarrow \boldsymbol{w}_{d}(t), t \geq 0
$$

along desired trajectories $\boldsymbol{w}_{d}(t)=\left[w_{1, d}(t), w_{2, d}(t)\right]^{T}$ for the realization of e.g. startup, set point changes, transient operation, or shutdown of the tubular reactor.

Therefore, it can be observed that the considered tubular reactor model (2.61)-(2.64) with boundary inputs directly corresponds to the DCR-system structure introduced for the derivation of Theorem 2.4, whereby $R_{0}=\mathrm{O}_{2}$ and $P_{0}=R_{1}=Q=\mathrm{I}_{2}$ with $\mathrm{O}_{2}$ and $\mathrm{I}_{2}$ the $2 \times 2$ zero and unit matrix, respectively. Assuming that the vector $\boldsymbol{\Psi}(\boldsymbol{x}, \partial \boldsymbol{x} / \partial z)$ can be transferred into the form (2.45), the tubular reactor can be parameterized by a formal power series (2.43) in terms of a parameterizing function $\boldsymbol{y}(t)=F_{d} \frac{\partial \boldsymbol{x}}{\partial z}(0, t)+F_{v} \boldsymbol{x}(0, t)$ if the matrix $\Theta$ defined by (2.60), i.e.

$$
\Theta=\left(\begin{array}{ccccc}
0 & 0 & \vdots & 1 & 0 \\
0 & 0 & \vdots & 0 & 1 \\
\hdashline & . & 0 & 0 & . \\
F_{v} & \vdots & & F_{d}
\end{array}\right)
$$

is non-singular. Since $\operatorname{det} \Theta=\operatorname{det} F_{v}$, the tubular reactor model (2.61)-(2.64) is formally parameterizable whenever $F_{v}$ is non-singular. Hence a family of imposed BCs (2.50) exists, namely $\boldsymbol{y}(t)=F_{d} \frac{\partial \boldsymbol{x}}{\partial z}(0, t)+F_{v} \boldsymbol{x}(0, t)$ with $\operatorname{det} F_{v} \neq 0$. Consequently, there is no unique solution to the parameterization problem. However, the degrees of freedom in the choice of $F_{v}$ with $\operatorname{det} F_{v} \neq 0$ for arbitrary $F_{d}$ allow to select the most suitable parameterizing function $\boldsymbol{y}(t)$ for the given control problem. In case of the tubular reactor example, the most natural choice is given by $F_{d}=\mathbb{O}_{2}$ and $F_{v}=\mathbb{I}_{2}$ such that $\boldsymbol{y}(t)$ corresponds to the output variables $\boldsymbol{y}(t)=$ $\boldsymbol{x}(0, t)=\boldsymbol{w}(t)$. $^{8}$

For the application of the formal power series approach, it is required to justify assumption (2.45) for the convective and reactive terms of the model. Note that this imposed restriction

\footnotetext{
${ }^{8}$ In the following $\boldsymbol{y}(t)$ and $\boldsymbol{w}(t)$ are equivalently used to express the output of the reactor.
} 
is rather weak, since many types of nonlinearities can at least within a subset of their range of definition be expressed or approximated by a formal power series of the above form (Meurer and Zeitz, 2003b). For the vector $\boldsymbol{\Psi}(\boldsymbol{x}, \partial \boldsymbol{x} / \partial z)$ of nonlinearities (2.66), the following expansion of the exponential term holds locally with constants $a_{i}, i=0,1, \ldots, I$ adjusted for a given range of operation for $x_{2}(z, t)$

Assumption 2.2. There exist constants $a_{i} \in \mathbb{R}, i=0,1, \ldots, I$, s.t.

$$
\exp \left(\frac{x_{2}}{1+x_{2} / \sigma}\right) \approx \sum_{i=0}^{I} a_{i} x_{2}^{i}
$$

at least locally within some range of values of $x_{2}$.

Consequently, evaluation of (2.66) with (2.70) provides that

$$
\boldsymbol{\Psi}\left(\boldsymbol{x}, \frac{\partial \boldsymbol{x}}{\partial z}\right)-\left[\begin{array}{c}
L e \frac{\partial x_{1}}{\partial z} \\
\frac{\partial x_{2}}{\partial z}-\beta x_{2}
\end{array}\right] \approx\left[\begin{array}{c}
0 \\
\beta x_{2 w}
\end{array}\right]+\left[\begin{array}{c}
D a L e \\
B D a
\end{array}\right]\left(1-x_{1}\right) \sum_{i=0}^{I} a_{i} x_{2}^{i}=: \boldsymbol{\varphi}(\boldsymbol{x})
$$

which can be transferred in the required form (2.45), (2.46) by successive application of the Cauchy product formula to $\boldsymbol{\varphi}(\boldsymbol{x})$, i.e.

$$
\boldsymbol{\varphi}\left(\sum_{n=0}^{\infty} \hat{\boldsymbol{x}}_{n} z^{n}\right)=\sum_{n=0}^{\infty} \hat{\boldsymbol{\varphi}}_{n}\left(\underline{\hat{\boldsymbol{x}}}_{n}\right) z^{n} .
$$

Note that the remaining constants in (2.71) $\beta x_{2 w}$ and $a_{0} D a L e$ or $a_{0} B D a$ respectively, do not complicate the analysis since they only appear in the leading coefficients of $z^{0}$ of the formal power series expansion.

As a result, the differential recursions for the determination of the series coefficients $\hat{\boldsymbol{x}}_{n}(t)=$ $\left[\hat{x}_{1, n}(t), \hat{x}_{2, n}(t)\right]^{T}$ of the formal power series (2.43) can be determined from (2.47) for any $n \in \mathbb{N}_{0}$

$$
\begin{aligned}
& \hat{x}_{1, n+2}=\frac{P e_{1}}{(n+2)(n+1)}\left(\frac{1}{L e} \frac{d \hat{x}_{1, n}}{d t}-(n+1) \hat{x}_{1, n+1}-\frac{1}{L e} \hat{\varphi}_{1, n}\left(\underline{\hat{x}}_{n}\right)\right) \\
& \hat{x}_{2, n+2}=\frac{P e_{2}}{(n+2)(n+1)}\left(\frac{d \hat{x}_{2, n}}{d t}-(n+1) \hat{x}_{2, n+1}+\beta \hat{x}_{2, n}-\hat{\varphi}_{2, n}\left(\underline{\hat{\boldsymbol{x}}}_{n}\right)\right) .
\end{aligned}
$$

Their evaluation requires the specification of two starting conditions for $\hat{\boldsymbol{x}}_{0}(t)$ and $\hat{\boldsymbol{x}}_{1}(t)$ from the BCs (2.62) and the imposed boundary conditions with $F_{d}=\mathbb{O}_{2}$ and $F_{v}=\mathbb{I}_{2}$ such that

$$
\begin{aligned}
& \hat{\boldsymbol{x}}_{0}(t)=\left[\hat{x}_{1,0}(t), \hat{x}_{2,0}(t)\right]^{T}=\boldsymbol{y}(t)=\boldsymbol{x}(0, t) \\
& \hat{\boldsymbol{x}}_{1}(t)=\left[\hat{x}_{1,1}(t), \hat{x}_{2,1}(t)\right]^{T}=\mathbf{0}=\frac{\partial \boldsymbol{x}}{\partial z}(0, t) .
\end{aligned}
$$

Under the assumption of sufficiently smooth functions $\boldsymbol{y}(t) \in \mathcal{C}^{\infty}$, the differential recursions (2.72), (2.73) with starting conditions (2.74), (2.75) are uniquely solvable by sequential evaluation. Note that the complexity of the recursions prevents a closed-form solution. Since 
$\hat{\boldsymbol{x}}_{0}(t)=\boldsymbol{y}(t)$ and $\hat{\boldsymbol{x}}_{1}(t)=\mathbf{0}$, the formal power series ansatz (2.43) can be parameterized by $\boldsymbol{y}(t)$ which in analogy to (2.57) yields a schematic representation

$$
\begin{aligned}
\hat{\boldsymbol{x}}(z, t) & =\sum_{n=0}^{\infty}\left[\boldsymbol{\Upsilon}_{2 n}\left(\boldsymbol{y}(t), \dot{\boldsymbol{y}}(t), \ldots, \boldsymbol{y}^{(n)}(t)\right) z^{2 n}+\boldsymbol{\Upsilon}_{2 n+1}\left(\boldsymbol{y}(t), \dot{\boldsymbol{y}}(t), \ldots, \boldsymbol{y}^{(n)}(t)\right) z^{2 n+1}\right] \\
& =\boldsymbol{\Upsilon}\left(z, \boldsymbol{y}(t), \dot{y}(t), \ldots, \boldsymbol{y}^{(n)}(t), \ldots\right) .
\end{aligned}
$$

For the parameterization of the inputs $\boldsymbol{u}(t)$, formally evaluate boundary condition (2.62) with (2.67) and the series ansatz (2.43) for $i=1,2$

$$
\hat{u}_{i}(t)=\sum_{n=0}^{\infty}\left(\hat{x}_{i, n}(t)+\frac{(n+1)}{P e_{i}} \hat{x}_{i, n+1}(t)\right) .
$$

Utilizing (2.54), (2.55) for the considered problem provides the desired expression $\hat{\boldsymbol{u}}(t)$ for the series expansion of the input functions $\boldsymbol{u}(t)$ in terms of the parameterizing functions $\boldsymbol{y}(t)$ and their time-derivatives up to infinite order. After re-arrangement of the series coefficients, this yields

$$
\begin{aligned}
\hat{u}_{i}(t)= & y_{i}(t)+\sum_{n=0}^{\infty}\left[1+\frac{2 n+1}{P e_{i}}\right] \Upsilon_{i, 2 n+1}\left(\boldsymbol{y}(t), \ldots, \boldsymbol{y}^{(n)}(t)\right) \\
& +\sum_{n=1}^{\infty}\left[1+\frac{2 n}{P e_{i}}\right] \Upsilon_{i, 2 n}\left(\boldsymbol{y}(t), \ldots, \boldsymbol{y}^{(n-1)}(t), y_{i}^{(n)}(t)\right)
\end{aligned}
$$

As can be clearly seen from the parameterization (2.76), series convergence greatly depends on an appropriate choice of trajectories for $\boldsymbol{y}(t)$. Hence if convergence can be ensured, (2.78) immediately provides the open-loop controls $\boldsymbol{u}_{d}(t)=\left[u_{1, d}(t), u_{2, d}(t)\right]^{T}$ which are required to track the prescribed output trajectories $\boldsymbol{y}(t) \rightarrow \boldsymbol{y}_{d}(t)$ in the nominal case. In particular uniform convergence is required to validate the so far formal operations such as the interchange of differentiation and summation. Exemplarily uniform convergence is proven for the case of $I=1$ in (2.70), i.e. first order approximation of the Arrhenius reaction term.

Theorem 2.5. For the first order approximation of the reaction kinetics (2.70), the series solution (2.76) with coefficients satisfying the differential recursions (2.72)-(2.73) converges with at least a unit radius of convergence if $y_{i}(t), i=1,2$ is Gevrey of order $\alpha \leq 2$ with constants $M_{i}, i=1,2, R_{1}=R_{2}=R$ and if the following inequalities are satisfied

$$
\begin{aligned}
P e_{1} & \leq \frac{2}{1+\frac{2}{R L e}+D a\left(\left|a_{0}\right|+\left|a_{1}\right| M_{2}\left(1+\frac{1}{M_{2}}\right)\right)} \\
P e_{2} & \leq \frac{2}{1+\frac{2}{R}+B D a M_{1}\left(\left|a_{1}\right|+\frac{\left|a_{0}\right|}{M_{2}}\right)+\left|\left(\beta-a_{1} B D a\right)\right|} .
\end{aligned}
$$

Note that one could as well replace $M_{i}, i=1,2$ by $M=\max \left\{M_{1}, M_{2}\right\}$. The resulting inequalities represent rather severe restrictions on the application of the formal solution. In particular, 
Eqns. (2.79), (2.80) yield that $P e_{i} \in(0,2]$, i.e. the convective effects must be dominated by the diffusive effects.

The proof follows the lines of the proof of Theorem 2.3 and is therefore just sketched below.

Proof. By induction, it is shown that

$$
\sup _{t \in \mathbb{R}^{+}}\left|\hat{x}_{i, n}^{(l)}(t)\right| \leq \frac{M_{i} \epsilon^{n}}{R^{l}} \frac{(n+l) !^{\alpha}}{n !^{\alpha}}, \quad i=1,2 .
$$

For $I=1$ in (2.70), the application of Lemmas A.2 and A.3 of the Appendix A yields after some intermediate computations similar to those of the proof of Theorem 2.3 that

$$
\begin{aligned}
\frac{1}{P e_{1}}\left|\hat{x}_{1, n}^{(l)}\right| & \leq \underbrace{\frac{M_{1} \epsilon^{n}(n+l) !^{\alpha}}{R^{l} n !^{\alpha}}\left[\frac{1}{R L e \epsilon^{2}}+\frac{\epsilon+D a\left(\left|a_{0}\right|+M_{2}\left|a_{1}\right|\left[1+\frac{1}{M_{1}}\right]\right)}{2 \epsilon^{2}}\right]}_{=: h_{1}(\epsilon)} \\
\frac{1}{P e_{2}}\left|\hat{x}_{2, n}^{(l)}\right| \leq & \leq \underbrace{\frac{M_{2} \epsilon^{n}(n+l) !^{\alpha}}{R^{l} n !^{\alpha}}[\frac{1}{R \epsilon^{2}}+\underbrace{\epsilon+B D a M_{1}\left(\left|a_{1}\right|+\frac{\left|a_{0}\right|}{M_{2}}\right)+\left|\left(\beta-a_{1} B D a\right)\right|}}_{=: h_{2}(\epsilon)} .
\end{aligned}
$$

From the Cauchy-Hadamard formula for $\alpha \in[1,2]$, the radii of convergence $\rho_{i}$ of the formal power series $\sum_{n} \hat{x}_{i, n}(t) z^{n}, i=1,2$ with the assumed bounds are given by $\rho_{1}=\rho_{2}=\frac{1}{\epsilon}$. Hence solving each $h_{i}(\epsilon)=\frac{1}{P e_{i}}, i=1,2$ and taking the respective maximal $\epsilon$ yields the inequalities (2.79), (2.80).

The following remark illustrates extensions of the derived results for the boundary controlled tubular reactor to input and output delays, which partly arise in applications.

Remark 2.2. The proposed approach for feedforward control design using formal power series can be easily extended to several scenarios involving input and output delays:

- In case of input delays, i.e. when the input is given as $\boldsymbol{u}\left(t-\Delta t_{u}\right)$, the input parameterization (2.78) can be evaluated at $t+\Delta t_{u}$, which directly provides the compensation of the delay $\Delta t_{u}$

- In case of a product recycle loop (Antoniades and Christofides, 2001; Berezowski, 2001), a ratio $r$ of the product stream at the outlet of the reactor is fed back time-delayed into the reactor inlet, such that boundary condition (2.62) reads $P_{1} \frac{\partial \boldsymbol{x}}{\partial z}(1, t)+\boldsymbol{x}(1, t)=(1-$ $r) \boldsymbol{u}_{r}(t)+r \boldsymbol{y}\left(t-\Delta t_{y}\right)$. Since the reactor output $\boldsymbol{y}(t)$ denotes the parameterizing function, simply choose $\boldsymbol{u}_{r}(t)=\left(\boldsymbol{u}(t)-r \boldsymbol{y}\left(t-\Delta t_{y}\right)\right) /(1-r)$ with $\boldsymbol{u}(t)$ from (2.78) to compensate the influence of the delayed output due to the recycle loop. 
For the computation of the series coefficients $\hat{\boldsymbol{x}}_{n}, n \geq 0$ by sequential evaluation of the differential recursions (2.72)-(2.75), efficient symbolic algorithms - see Appendix C - were developed and implemented in the computer algebra system MaтнематісA (Wolfram, 1999). As a result, analytical expressions for the series coefficients up to high orders are obtained, which can be used for evaluation of the formal power series.

As illustrated for scalar linear and nonlinear DCR-equations with boundary control in Section 2.1 as well as general systems of DCR-equations in Section 2.2, formal power series - if applicable - provide a useful approach to parameterize system states and boundary inputs in terms of a parameterizing function which typically represents a combination of boundary state values. In case of uniform convergence which can be ensured by appropriate motion planning, this parameterization directly yields the open-loop control to realize the desired motion. This underlines that controllability and parameterizability interact, whereby the latter can be interpreted as a constructive controllability analysis.

This property can be further generalized and leads to the introduction of the notion of formal power series parameterizability, which provides a general framework to embed the analysis carried out exemplarily for the linear heat and diffusion equation as well as scalar and systems of nonlinear DCR-equations.

\subsection{Formal power series parameterizability, flatness and controllability of DCR-systems}

As illustrated in the previous sections, formal power series provide a widely applicable approach for motion planning and feedforward control design for boundary controlled DCRsystems. Hence a generalization of the parameterizing procedure outlined above seems appropriate. Therefore, the notion of formal power series parameterization (FPSP) is introduced (Meurer and Zeitz, 2004a; Wagner et al., 2004; Meurer and Zeitz, 2005), which extends these ideas to rather general nonlinear sets of DCR-equations.

Definition 2.1 (Formal Power Series Parameterization). An $K$-dimensional set of nonlinear diffusion-convection-reaction equations with boundary inputs

$$
\begin{gathered}
G_{i}\left(\frac{\partial \boldsymbol{x}(z, t)}{\partial t}, \boldsymbol{x}(z, t), \frac{\partial \boldsymbol{x}(z, t)}{\partial z}, \frac{\partial^{2} \boldsymbol{x}(z, t)}{\partial z^{2}}\right)=0, \quad z \in(0,1), \quad t>0, \quad i=1, \ldots, K \\
R_{0, j}\left(\boldsymbol{x}(0, t), \frac{\partial \boldsymbol{x}}{\partial z}(0, t), \boldsymbol{u}_{0}(t)\right)=0, \quad t>0, \quad j=1, \ldots, K_{0} \\
R_{1, l}\left(\boldsymbol{x}(1, t), \frac{\partial \boldsymbol{x}}{\partial z}(1, t), \boldsymbol{u}_{1}(t)\right)=0, \quad t>0, \quad l=1, \ldots, K_{1} \quad\left(K_{0}+K_{1}=2 K\right) \\
\boldsymbol{x}(z, 0)=\boldsymbol{x}_{0}(z), \quad z \in[0,1]
\end{gathered}
$$


defined on $(z, t) \in[0,1] \times \mathbb{R}^{+}$with state vector $\boldsymbol{x}(z, t)=\left[x_{1}(z, t), \ldots, x_{K}(z, t)\right]^{T}$ and input $\boldsymbol{u}(t)$ from suitable functional spaces $\mathcal{X}[0,1]$ and $\mathcal{U}$, respectively, is called formal power series parameterizable (FPSP), if there exists a formal power series

$$
\hat{\boldsymbol{x}}(z, t)=\sum_{n=0}^{\infty} \hat{\boldsymbol{x}}_{n}(t) p_{n}(z),
$$

in $p_{n}(z)$, an appropriate polynomial of degree $n$ in $z$, which formally satisfies (2.81)-(2.84) and whose coefficients $\hat{\boldsymbol{x}}_{n}(t)$ can be expressed in terms of a parameterizing function

$$
\boldsymbol{y}(t)=\boldsymbol{H}\left(\boldsymbol{x}(0, t), \frac{\partial \boldsymbol{x}}{\partial z}(0, t), \boldsymbol{x}(1, t), \frac{\partial \boldsymbol{x}}{\partial z}(1, t), \boldsymbol{u}_{0,1}(t)\right)
$$

with $\operatorname{dim} \boldsymbol{y}=\operatorname{dim} \boldsymbol{u}_{0}+\operatorname{dim} \boldsymbol{u}_{1}$ and its time-derivatives i.e.

$$
\hat{\boldsymbol{x}}_{n}(t)=\boldsymbol{\Upsilon}_{n}\left(\boldsymbol{y}(t), \dot{\boldsymbol{y}}(t), \ldots, \boldsymbol{y}^{\left(r_{x}\right)}(t)\right)
$$

where $r_{x} \in \mathbb{N}_{0}$ and possibly $r_{x} \rightarrow \infty$ as $n \rightarrow \infty$. The system inputs $\boldsymbol{u}_{0,1}(t)$ are called formal power series parameterizable, if (2.81)-(2.84) are formally power series parameterizable and (2.82), (2.83) can be (explicitly) solved for $\boldsymbol{u}_{0,1}(t)$.

This definition basically expresses the possibility to parameterize the state $\boldsymbol{x}(z, t)$ and the inputs $\boldsymbol{u}_{0,1}(t)$ of a given DCR-system via a formal power series in terms of a parameterizing function $\boldsymbol{y}(t)$.

Hence, formal power series parameterizability resembles the notion of differential flatness as introduced for nonlinear finite dimensional systems by Fliess et al. (1995). In particular note that no general extension of differential flatness is so far available for DPS, whereby several authors (Aoustin et al., 1997; Fliess et al., 1997, 1998a,b; Laroche et al., 1998; Laroche and Martin, 2000; Laroche et al., 2000; Lynch and Rudolph, 2002; Ollivier and Sedoglavic, 2001; Petit, 2002; Rudolph, 2003a) adopt the flatness notion to express the possibility to parameterize a given DPS and hence determine the corresponding inverse system representation. Initial steps to extend the flatness notion to DPS are proposed by Fliess et al. (1997); Laroche (2000); Rouchon (2001). Thereby, the results of Laroche (2000) for boundary controlled linear parabolic DPS are also based on a series ansatz with two essential differences to FPSP:

(i) The series is defined in terms of known temporal behavior with the spatial part being determined by sequential solution of boundary-value problems (BVPs) depending on the respective BCs of the governing linear DPS.

(ii) The series has to converge uniformly.

The notion of FPSP generalizes these ideas, whereby in particular uniform convergence is not a priori imposed. As will be seen in the following chapters, this requirement can be relaxed by considering possibly divergent series which are summable in a certain to be defined sense. 
On the other hand and as illustrated in Section 2.1 for linear and nonlinear scalar equations as well as Section 2.2 for the more general setup, the determination of the parameterization provides a direct construction of the boundary inputs $\boldsymbol{u}(t)$ necessary to track the imposed outputs $\boldsymbol{y}(t)$ in open-loop, assuming convergence (or summability as will be seen later) of the obtained parameterized power series. Hence existence of a parameterization is related to the controllability analysis.

For DPS, there is basically a distinction between the following three controllability notions (Russell, 1978; Curtain and Zwart, 1995; Zuazua, 2004):

- Exact controllability in finite time $T>0$, i.e. given any initial and final state, $\boldsymbol{x}_{0}(z), \boldsymbol{x}_{T}(z) \in \mathcal{X}[0,1]$ there exists an input $\boldsymbol{u}(t) \in \mathcal{U}$ such that the solution of (2.81)-(2.84) satisfies $\boldsymbol{x}(z, T)=\boldsymbol{x}_{T}(z)$,

- Approximate controllability in finite time $T>0$, i.e. any final state $\boldsymbol{x}_{t}(z)$ can be reached up to an $\epsilon$-neighborhood,

- Null-controllability, i.e. the terminal state is the zero state $\boldsymbol{x}_{T}(z) \equiv 0$.

Note that approximate and null-controllability are the natural notions for parabolic DPS (Zuazua, 2004), where in particular many general results are available for the linear case - see e.g. Russell (1978); Curtain and Zwart (1995); Zuazua (2004) and the references therein. On the other hand, for nonlinear parabolic DPS only few results are available. Thereby, Doubova et al. (2002) verify approximate and null-controllability for systems similar to the boundary controlled DCR-system (2.39)-(2.42) by imposing growth restrictions on the nonlinear term $\boldsymbol{\Psi}(\boldsymbol{x}, \partial \boldsymbol{x} / \partial z)$.

In any case, the notion of controllability is related to the existence of a control without its explicit computation. Alternatively within the parameterization approach, Laroche (2000) introduces the notion of 'commandabilité approchée plat' in the sense of a constructive verification of controllability providing explicit solutions to the control problem. For the illustration of this notion, consider a linear scalar DCR-equation, which exhibits a uniformly convergent state and input parameterization in terms of a parameterizing function $y(t)$. Let $\mathcal{O}$ denote the set of initial and final states of the DCR-equation, which can be connected by an appropriate choice of $y(t)$. Furthermore let $u^{y}(t) \in \mathcal{C}^{\infty}(0, T)$ denote the parameterized input in terms of $y(t)$ which steers the DPS - in an exact sense - from a given initial state $x_{0}^{y} \in \mathcal{O}$ to a given finite state $x_{T}^{y} \in \mathcal{O}$ in finite time $T>0$. Then it can be proven (Laroche, 2000, Prop. 2.6.2), that given initial and final states $x_{0}$ and $x_{T}$ of the DPS satisfying $\left\|x_{i}-x_{i}^{y}\right\|_{\mathcal{X}} \leq \epsilon, i=0, T$, there exists a control $u(t) \in \mathcal{C}^{2}(0, T)$ and a constant $K(T)>0$, such that $\left\|x(\cdot, T)-x_{T}\right\|_{\mathcal{X}} \leq K(T)$ starting from $x(\cdot, 0)=x_{0}$. Hence, parameterizability implies approximate controllability.

Although no proof of this result is available for more general and in particular nonlinear problems, in the following chapters, the proposed parameterization approach will serve as a constructive illustration of controllability for rather general nonlinear systems of boundary controlled DCR-equations. 


\subsection{Conclusions}

In this chapter, the property of formal power series parameterizability is introduced as a widely applicable parameterization-based tool for feedforward control design and motion planning for boundary controlled nonlinear DCR-systems. Thereby it can be shown that FPSP is directly related to controllability in a constructive sense providing explicit solutions to the control problem. Based on various examples including the linear heat and diffusion-convection equation or the model of a non-isothermal tubular fixed-bed reactor, it is illustrated that the main drawback of this approach is hidden in the proof of convergence of the resulting series representations for system states and inputs. Hence, a more general framework is desired to overcome these problems. 


\section{Chapter 3}

\section{Summability methods and sequence transformations}

In the previous chapter, (formal) power series are used to determine the inverse system representation of a given boundary controlled PDE in terms of a parameterizing function and its derivatives up to infinite order. Thereby, convergence of the series solution greatly depends on appropriate motion planning and the structure of the considered system, which significantly restricts the applicability of method.

One approach to overcome these limitations is provided by so-called summability methods (Borel, 1928; Hardy, 1949; Ramis, 1980; Malgrange, 1995; Balser, 1994, 2000), with the desire to prolong the space of uniformly convergent power series in order to deal with certain divergent series. Within this framework, general results are available for formal solutions to ODEs (Ramis, 1980; Malgrange, 1995; Balser, 1994, 2000), and recent work extends these ideas to the study of PDEs (Lutz et al., 1996; Balser and Miyake, 1999; Balser, 2001). Nevertheless, since many practical problems typically allow only the determination of a finite number of series coefficients, these theoretically appealing approaches have to be suitably modified, which leads to so-called generalized sequence transformations (GST) (Brezinski, 1977; Smith and Ford, 1979; Weniger, 1989). These methods allow to accelerate convergence and approximate the sum of certain divergent series using only partial information on the series under consideration and in particular play a crucial role e.g. in quantum physics and quantum chemistry (Weniger, 1994).

In order to motivate these approaches and to build a mathematical basis for the presented ideas, in this chapter formal power series with coefficients in some space of functions in one or more variables - here a fixed but arbitrary Banach space $\mathbb{E}$ equipped with some fixed norm $\|\cdot\|$ over the field of complex numbers $\mathbb{C}$ - are considered following mainly the lines of Balser (2000). This is in particular necessary for the study of formal solutions of PDEs. Hence, after briefly summarizing some mathematical background in Section 3.1, formal power series and their interpretation as asymptotic expansions are considered in Section 3.2. By imposing a certain growth condition on their remainder terms, so-called Gevrey asymptotic expansions can be introduced, which naturally lead to the definition of Gevrey functions as 
already considered in Chapter 2. Based on these results, summation methods are introduced in Section 3.3, which allow to uniquely determine a respective holomorphic function given a possibly divergent formal power series. Thereby, it is in particular desired to prolong the space of convergent series, while retaining its favorable algebraic properties. This leads to Borel and $k$-summation (Borel, 1928; Ramis, 1980), whose ability to sum divergent series is exemplarily studied for the geometric and Euler series. In view of the numerical implementation and consideration of practical problems, certain modifications are proposed, which result in the so-called $(N, \xi)$-approximate $k$-summation (Wagner, 2003), (Wagner et al., 2004), whose properties are exemplified for the geometric and Euler series in Section 3.4. Finally, iterative and nonlinear GSTs are considered in Section 3.5 for comparison purposes and to illustrate their ability to accelerate convergence and to sum certain divergent series.

\subsection{Preliminaries}

For the sake of completeness and to make this chapter self-contained, some fundamental mathematical preliminaries have to be briefly introduced, which are essential for the study of formal power series over the field of complex numbers, asymptotic expansions, and summability methods (Balser, 2000).

Definition 3.2 (Sector). A set $S=S(d, \varsigma, \sigma)=\{z: 0<|z|<\sigma,|d-\arg z|<\varsigma / 2\}$ on the Riemann surface of the logarithm ${ }^{1}$ with $d \in \mathbb{R}$ (bisecting direction), $\varsigma \in \mathbb{R}^{+}$(bisecting opening), $\sigma \in \mathbb{R}^{+}$(bisecting radius) or $\sigma=\infty$ is called a sector.

If $\sigma<\infty$ or $\sigma=\infty$ the sector is of finite or infinite radius, respectively. If $\sigma=\infty$, then $S(d, \varsigma)=S(d, \varsigma, \sigma)$.

Definition 3.3 (Closed sector). A closed sector is a set of the form $\bar{S}=\bar{S}(d, \varsigma, \sigma)=$ $\{z: 0<|z| \leq \sigma,|d-\arg z| \leq \varsigma / 2\}$ with $\sigma \in \mathbb{R} \neq \infty$.

Obviously, a closed sector never includes the origin and is always of finite radius, such that closed sectors are not closed as subsets of $\mathbb{C}$ but are as subsets of the Riemann surface of the logarithm.

Definition 3.4 (Sectorial region). A region $\Omega(d, \varsigma)$ on the Riemann surface of the logarithm, is called a sectorial region if $\exists d, \varsigma \in \mathbb{R}^{+}: \Omega \subset S(d, \varsigma)$, while $\forall \beta: 0<\beta<\varsigma \exists \sigma>0$ : $\bar{S}(d, \beta, \sigma) \subset \Omega$.

\footnotetext{
${ }^{1}$ Let $z \in \mathbb{C}$ such that $z=r e^{\imath \varphi}$ and let $0<r \leq 1$, then $\log z=\log r+\imath \varphi$. By varying $r$ and $\varphi$ one obtains a spiraling staircase, the Riemann surface of the logarithm, over the complex plane $|z| \leq r$ with infinitely many levels in both directions centered at the origin. Any point on this staircase can be projected onto the complex plane $\mathbb{C}$, say to a point $z=p e^{\imath \psi}$. Thereby the points $z$ and $z e^{2 \pi \imath}$ coincide in $\mathbb{C}$ but are not the same on the staircase due to their different distance to the origin - the points sit on each other. Hence on the Riemann surface, the argument of a point is uniquely defined, such that no distinction between the possibly infinite number of branches of a function is necessary.
} 

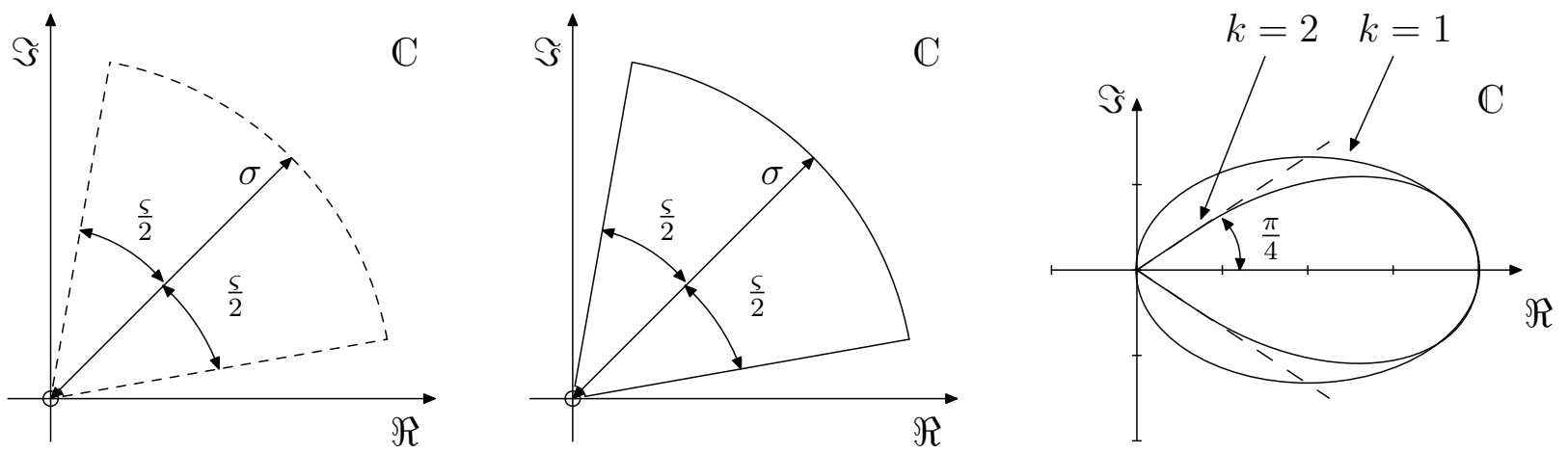

Figure 3.1: Example $\mathbb{E}=\mathbb{C}$ : sector $S(d, \varsigma, \sigma)$ as the interior of the area limited by the dashed lines (left); closed sector $\bar{S}(d, \varsigma, \sigma)$ as the union of interior and boundary of the marked area (middle); sectorial region $\Omega$ of opening $\pi / k$ defined by the inequalities $k|d-\arg z|<\pi / 2 \wedge \cos (k[d-\arg z])>$ $c|z|^{k}$ for $d=0, c=1$, and $k \in\{1,2\}$ (right).

Thereby $\varsigma$ is called the opening and $d$ the bisection direction of $\Omega$. Let $\Omega$ be a given sectorial region and let $x \in \boldsymbol{H}(\Omega, \mathbb{E})$, i.e. the set of all $\mathbb{E}$-valued functions holomorphic in a sectorial region $\Omega$. Note that $x$ might be multi-valued if $\Omega$ has an opening greater than $2 \pi$.

Definition 3.5 (Holomorphic function). The function $x: \Omega \rightarrow \mathbb{E}$ is called holomorphic in $\Omega$ if for every $z_{0} \in \Omega$ the limit

$$
\lim _{z \rightarrow z_{0}}\left[z-z_{0}\right]^{-1}\left[x(z)-x\left(z_{0}\right)\right]=x^{\prime}\left(z_{0}\right)
$$

exists.

For functions $x \in \boldsymbol{H}(\Omega, \mathbb{E})$, properties such as boundedness, continuity (see Appendix A) and exponential growth can be defined, which play a crucial role for the study of formal power series in $z \in \mathbb{C}$. In particular, the property of exponential growth of a function is essential to study summability of a given formal power series.

Definition 3.6 (Exponential growth). Let $S=S(d, \varsigma)$ be a sector of infinite radius, and let $x(z)$ be holomorphic in $S$. Suppose that there exists $k>0$ for which the following holds true: To every $\varphi$ with $|d-\varphi|<\varsigma / 2, \exists \rho, c_{1}, c_{2}>0$ s.t. for every $z$ with $|z| \geq \rho,|d-\arg z| \leq \varphi$,

$$
\|x(z)\| \leq c_{1} \exp \left(c_{2}|z|^{k}\right)
$$

Then the function $x(z)$ is said to be of exponential growth at most $k$ in $S$.

In the following, the set of functions $x$ being holomorphic and of exponential growth at most $k$ in a sector $S$ and continuous at the origin shall be denoted by $\boldsymbol{A}^{(k)}(S, \mathbb{E})$. 


\subsection{Asymptotic power series and Gevrey functions}

In the following, formal power series with coefficients from some Banach space and asymptotic expansions are considered in order to motivate the application of suitable summation methods.

\subsubsection{Formal power series on Banach spaces}

Consider a sequence $\left\{\hat{x}_{n}\right\}_{n=0}^{\infty}$ of elements of the Banach space $\mathbb{E}$ and an element $z \in \mathbb{C}$.

Definition 3.7 (Formal power series). Given a sequence $\left\{\hat{x}_{n}\right\}_{n=0}^{\infty}$ of elements of $\mathbb{E}$, the series

$$
\hat{x}(z)=\sum_{n=0}^{\infty} \hat{x}_{n} z^{n}
$$

is called a formal power series (in $z \in \mathbb{C}$ ).

The term "formal" simply reflects the fact that the radius of convergence of the power series (3.1) might eventually be equal to zero. The set of all such formal power series will be denoted by $\mathbb{E}[[z]]$. The properties of the set $\mathbb{E}[[z]]$ can be determined by introducing the natural operations on $\mathbb{E}[[z]]$ i.e.

(1) addition:

$$
\oplus: \mathbb{E}[[z]] \times \mathbb{E}[[z]] \rightarrow \mathbb{E}[[z]] \text { s.t. } \hat{w}(z) \oplus \hat{x}(z)=\sum_{n=0}^{\infty}\left(\hat{w}_{n}+\hat{x}_{n}\right) z^{n}
$$

(2) scalar multiplication:

$$
\odot: \mathbb{E} \times \mathbb{E}[[z]] \rightarrow \mathbb{E}[[z]] \text { s.t } a \odot \hat{x}(z)=\sum_{n=0}^{\infty} a \cdot \hat{x}_{n} z^{n}
$$

(3) multiplication: ${ }^{2}$

$$
\circledast: \mathbb{E}[[z]] \times \mathbb{E}[[z]] \rightarrow \mathbb{E}[[z]] \text { s.t } \hat{w}(z) \circledast \hat{x}(z)=\sum_{n=0}^{\infty}\left(\sum_{j=0}^{n} \hat{w}_{n-j} * \hat{x}_{j}\right) z^{n}
$$

for $\hat{x}(z), \hat{w}(z) \in \mathbb{E}[[z]], a \in \mathbb{E}$ where $+, \cdot, *$ denote addition, scalar multiplication, and multiplication in the Banach space $\mathbb{E}$. If the context is clear, the binary composition laws $\oplus, \odot, \circledast$ as well as $\cdot$, * are omitted. It can be hence verified that the following properties hold true:

\footnotetext{
${ }^{2}$ It should be pointed out there exist various approaches to define series multiplication, such as Dirichlet's rule (Hardy, 1949; Knopp, 1964). In view of the following considerations on $k$-summation in Section 3.3, Cauchy's product rule is applied.
} 
(i) $\mathbb{E}[[z]]$ with respect to addition and scalar multiplication is a vector space over $\mathbb{C}$.

(ii) If $\mathbb{E}$ is a Banach algebra, then $\mathbb{E}[[z]]$ with respect to multiplication is an algebra over $\mathbb{C}$ and is commutative if $\mathbb{E}$ is commutative.

The proof of (i) follows directly from the assumption that $\mathbb{E}$ is a Banach space, i.e. a complete, normed linear space. Since (i) holds, the proof of property (ii) requires to verify that $\mathbb{E}[[z]]$ is a ring, and that scalar multiplication $\odot$ and multiplication $\circledast$ commute - see also Lang (1993). Furthermore it can be verified that $\mathbb{E}[[z]]$ has the structure of a differential algebra:

(iii) If $\mathbb{E}$ is a Banach algebra, then $\mathbb{E}[[z]]$ with respect to termwise differentiation (with respect to $z$ ) is a differential algebra over $\mathbb{C}$.

Further properties can be e.g. found in (Balser, 2000, p. 64f). These rather general and abstract results extend to the case when the formal power series $\hat{x}(z)$ is Gevrey of order $\alpha$. The importance of this notion is already outlined in Section 2.1 where functions of a certain Gevrey order became apparent for the proof of (uniform) convergence of the derived power series solutions.

Definition 3.8 (Gevrey order). The formal power series $\hat{x}(z)=\sum_{n=0}^{\infty} \hat{x}_{n} z^{n}$ is of Gevrey order $\alpha \geq 1$, if there exist positive $M, R$, such that

$$
\left\|\hat{x}_{n}\right\| \leq \frac{M}{R^{n}} \Gamma(1+(\alpha-1) n) \quad \forall n \in \mathbb{N}_{0} .
$$

Equivalent expressions can be derived from Hua and Rodino (1996), i.e.

$$
\left\|\hat{x}_{n}\right\| \leq \frac{M}{R^{n}}(n !)^{\alpha-1} \quad \text { or } \quad\left\|\hat{x}_{n}\right\| \leq \frac{M}{R^{n}} n^{(\alpha-1) n} \quad \forall n \in \mathbb{N}_{0} .
$$

Note that this definition slightly differs from the one in the classical literature on formal power series (Ramis, 1993; Malgrange, 1995; Balser, 2000), where the Gevrey order is defined in terms of $\check{\alpha}=\alpha-1$. Hence, a Gevrey order of 1 in the introduced notation equals a Gevrey order 0 in the cited literature. Nevertheless, the introduced notation is consistent with the literature on boundary control design using formal power series (Fliess et al., 1997; Laroche et al., 2000; Lynch and Rudolph, 2002; Rudolph, 2003a) and other authors dealing with PDEs in Gevrey classes (Hua and Rodino, 1996). Furthermore, this definition of Gevrey order directly carries over to the definition of a Gevrey function by considering asymptotic expansions as will be outlined below. In the sequel, the set of all formal power series of Gevrey order $\alpha \geq 1$ will be denoted by $\mathbb{E}[[z]]_{\alpha}$.

Since a simple proof shows that the composition laws $\oplus, \odot$, $\circledast$ as well as termwise differentiation (with respect to $z$ ) preserve the Gevrey order, the following property (Balser, 2000) follows from (i)-(iii):

(iv) The set $\mathbb{E}[[z]]_{\alpha}$ under the natural operations $\oplus, \odot$, $\circledast$ is a vector space closed under termwise differentiation. It is a differential algebra in case of $\mathbb{E}$ being a Banach algebra. 
Furthermore, the classical convergence notion for power series carries over to power series with coefficients in Banach spaces. The formal power series $\hat{x}(z)$ converges if there exists $\rho>0$, such that the power series converges for all $z$ with $|z|<\rho$ defining a function $x(z)$ (the sum of $\hat{x}$ ) holomorphic in a disc of radius $\rho$ centered at the origin. The set of all convergent power series will be denoted by $\mathbb{E}\{z\}$. Given a formal power series $\hat{x}(z) \in \mathbb{E}[[z]]_{\alpha}$, it follows by the Cauchy-Hadamard formula (A.1) that $\mathbb{E}[[z]]_{0}=\mathbb{E}\{z\}$.

\subsubsection{Asymptotic expansions}

It is well-know that series although possibly being divergent are still useful for numerical evaluations (Knopp, 1964). In order to illustrate this consider the Euler series (Hardy, 1949, p. 26) for real $z \geq 0$

$$
\hat{x}(z)=\sum_{n=0}^{\infty}(-1)^{n} n ! z^{n}
$$

whose radius of convergence equals zero. Euler summed this series to the function

$$
x(z)=\frac{e^{\frac{1}{z}}}{z} \int_{0}^{z} \frac{e^{-\frac{1}{\zeta}}}{\zeta} d \zeta .
$$

From the series, it can be easily observed that the series coefficients $\hat{x}_{n}(z):=(-1)^{n} n ! z^{n}$ for any $z<1$ first decrease (the series behaves like a convergent series) before growing beyond any bound. In view of this observation, values of $\hat{x}(z)$ at some $z<1$ can be computed with a prescribed accuracy, i.e. up to $d$ decimal places, by truncating the series at some fixed but yet unknown limit $N \in \mathbb{N}$. Of course, the possible accuracy depends on the value of $z$ and $N$, such that $d=d(z, N)$. In order to determine the necessary number of series coefficients required to obtain the requested accuracy, for this example, the first term of the remainder has to satisfy $(N+1) ! z^{N+1} \leq 10^{-d(z, N)} / 2$ for given $z<1$ (Knopp, 1964). For example let $z=0.1$ and request $d(0.1, N)=3$. Hence it follows from the inequality that $N=7$, which yields $\hat{x}^{(7)}(0.1)=\sum_{n=0}^{7}(-1)^{n} n !(0.1)^{n}=0.915416$ compared to the exact solution $x(0.1)=0.915633$ from (3.7). On the other hand $d(0.1, N)>3$ cannot be achieved since there does not exist an $N \in \mathbb{N}$ in order to fulfill the inequality. Nevertheless, a minimum deviation can be obtained also for this case by taking the $N$ for which the inequality reaches its minimum. This is the basic idea of the so-called least-term summation (Rudin, 1976), i.e.

$$
x(z) \cong \sum_{n=0}^{N} \hat{x}_{n} z^{n} \quad \text { with } N \text { from } \quad\left\|\hat{x}_{N} z^{N}\right\|=\min _{n \in \mathbb{N}_{0}}\left\{\left\|\hat{x}_{n} z^{n}\right\|\right\}, \quad z \in \Omega \subset \mathbb{C} .
$$

Obviously, the number $N$ of series coefficients is not a priori known, if existent at all. In anticipation of the introduction of summability methods, it can be shown that least-termsummation does not represent a (linear) summation method, but rather a heuristical approach to approximate the sum of a given series (Wagner, 2003). 
The considered example clearly illustrates, that a partial sum of given formal power series might possibly provide a sufficiently accurate approximation of the sum of the series at least in a neighborhood of the origin. These partial sums are called asymptotic expansions (Hardy, 1949; Knopp, 1964; Balser, 1994, 2000). The following definition is borrowed form Balser (2000) to account for formal power series with coefficients in Banach spaces.

Definition 3.9 (Asymptotic expansion). Given a function $x(z) \in \boldsymbol{H}(\Omega, \mathbb{E})$ and a formal power series $\hat{x}(z)=\sum_{n=0}^{\infty} \hat{x}_{n} z^{n} \in \mathbb{E}[[z]]$, then $\hat{x}(z)$ is the asymptotic expansion of $x(z)$ (or $x(z)$ asymptotically equals $\hat{x}(z))$ in $\Omega$, i.e. $x(z) \cong \hat{x}(z)$ in $\Omega$, if to every $N \in \mathbb{N}$ and every closed subsector $\bar{S}$ of $\Omega$ there exists $c=c(N, \bar{S})>0$ such that

$$
|z|^{-N}\left\|x(z)-\sum_{n=0}^{N-1} \hat{x}_{n} z^{n}\right\| \leq c \quad \text { for } z \in \bar{S} .
$$

This is equivalent to boundedness of the remainders

$$
R_{x}(z, N)=z^{-N}\left(x(z)-\sum_{n=0}^{N-1} \hat{x}_{n} z^{n}\right)
$$

at the origin for every $N \geq 0$.

Note that in case of $\hat{x}(z) \in \mathbb{E}\{z\}$, power series expansion and asymptotic expansion coincide. In addition, it can be proven, that the existence of an asymptotic expansion is equivalent to $x(z)$ being infinitely often differentiable at the origin $z=0$ (Malgrange, 1995; Balser, 2000).

Lemma 3.1. Let $x(z)$ be holomorphic in a sectorial region $\Omega$. Then the following statements are equivalent:

(i) $x(z) \cong \hat{x}(z)=\sum_{n=0}^{\infty} \hat{x}_{n} z^{n}$ in $\Omega$.

(ii) The function $x(z)$ is infinitely often differentiable at the origin, and $x^{(n)}(0)=n ! \hat{x}_{n}$ for $n \geq 0$.

(iii) All derivatives $x^{(n)}(z)$ are continuous at the origin and $x^{(n)}(z) \rightarrow n ! \hat{x}_{n}, \Omega \ni z \rightarrow 0, n \geq 0$.

In view of this Lemma, it can be shown that the set $\boldsymbol{A}(\Omega, \mathbb{E})$ of all functions $x \in \boldsymbol{H}(\Omega, \mathbb{E})$ having asymptotic expansion $\hat{x}(z)$ is a differential algebra under the natural operations $\oplus, \odot, \circledast$ assuming $\mathbb{E}$ is a Banach algebra (Balser, 2000). Furthermore

$$
J: \boldsymbol{A}(\Omega, \mathbb{E}) \rightarrow \mathbb{E}[[z]], \quad x \mapsto \hat{x}=J x
$$

mapping each $x$ to its asymptotic expansion is a surjective homomorphism between $\boldsymbol{A}(\Omega, \mathbb{E})$ and $\mathbb{E}[[z]]$. Hence, every asymptotic expansion in $\mathbb{E}[[z]]$ has an inverse image in $\boldsymbol{A}(\Omega, \mathbb{E})$. However, the map $J$ is not injective, i.e. there is no unique asymptotic expansion for a given holomorphic function. This property has further consequences. Assume that the formal power series $\hat{x}(z)$ 
satisfies a given differential equation. The above result ensures that there exists a holomorphic function $x(z)$ have $\hat{x}(z)=(J x)(z)$ as its asymptotic expansion. Nevertheless, since the map is not injective, it is not possible to conclude that $x(z)$ also solves the differential equation. For example, the functions $x_{1}(z)=\exp (-1 / z)$ and $x_{2}(z)=\exp \left(-1 / z^{2}\right)$ both have the same asymptotic expansion $\hat{x}(z)=0$ in the neighborhood of the origin $z=0$, with $x_{1}(z)$ solving the ODE $z^{2} x_{1}^{\prime}-x_{1}=0$ and $x_{2}(z)$ solving $z^{3} / 2 x_{2}^{\prime}-x_{2}=0$. Hence it is desired to work in a different set of asymptotic expansions which leads to the definition of so-called Gevrey asymptotics.

\subsubsection{Gevrey asymptotics}

In the previous section, asymptotic expansions are introduced by imposing quite arbitrary bounds on the remainder terms (3.9) or (3.10), respectively. Specifying a prescribed growth on the remainder terms in the sense of Definition 3.8, leads to Gevrey asymptotics (Ramis, 1993; Balser, 1994; Malgrange, 1995; Balser, 1994, 2000).

Definition 3.10 (Gevrey asymptotic expansion). Given $\alpha>1$, a function $x(z) \in$ $\boldsymbol{H}(\Omega, \mathbb{E})$, and a formal power series $\hat{x}(z)=\sum_{n=0}^{\infty} \hat{x}_{n} z^{n} \in \mathbb{E}[[z]]$, then $\hat{x}(z)$ is the asymptotic expansion of order $\alpha$ of $x(z)$ (or $x(z)$ asymptotically equals $\hat{x}(z)$ of Gevrey order $\alpha$ ) in $\Omega$, i.e. $x(z) \cong_{\alpha} \hat{x}(z)$ in $\Omega$, if to every $N \in \mathbb{N}$ and every closed subsector $\bar{S}$ of $\Omega$ there exists $M, R>0$ such that

$$
\left\|R_{x}(z, N)\right\|=\left\|z^{-N}\left(x(z)-\sum_{n=0}^{N-1} \hat{x}_{n} z^{n}\right)\right\| \leq \frac{M}{R^{N}} \Gamma(1+(\alpha-1) N) .
$$

Note that $x(z) \cong_{\alpha} \hat{x}(z)$ implies that $x(z) \cong_{\bar{\alpha}} \hat{x}(z)$ for $\bar{\alpha}>\alpha$. For $\alpha=1$, the function $x(z)$ is holomorphic at the origin with $\hat{x}(z)$ representing its power series expansion. Similar to Lemma 3.1, the following result can be proven (Balser, 2000).

Lemma 3.2. Let $x \in \boldsymbol{H}(\Omega, \mathbb{E})$, and let $\alpha \geq 1$. Then the following statements are equivalent:

(i) $x(z) \cong_{\alpha} \hat{x}(z)=\sum_{n=0}^{\infty} \hat{x}_{n} z^{n}$ in $\Omega$.

(ii) All derivatives $x^{(n)}(z)$ are continuous at the origin and for every closed subsector $\bar{S}$ of $\Omega$ there exist constants $M, R$ such that for every $n \in \mathbb{N}_{0}$

$$
\frac{1}{n !} \sup _{z \in \bar{S}}\left\|x^{(n)}(z)\right\| \leq \frac{M}{R^{n}} \Gamma(1+(\alpha-1) n) .
$$

This directly leads to the important notion of a Gevrey function (Hua and Rodino, 1996), which is already briefly introduced and utilized in Chapter 2 .

Definition 3.11 (Gevrey class). The function $x(z)$ is in $G_{M, R, \alpha}(\Omega)$, the Gevrey class of order $\alpha$ in $\Omega$, if $x(z) \in \mathcal{C}^{\infty}(\Omega)$ and for every closed subsector $\bar{S}$ of $\Omega$, there exist positive constants $M, R$ such that for all $\alpha$ and $z \in \bar{S}$

$$
\left\|x^{(n)}(z)\right\| \leq \frac{M}{R^{n}}(n !)^{\alpha} .
$$


Using multi-index notation, this definition directly carries over to the multi-dimensional case where $z \rightarrow \boldsymbol{z}=\left(z_{1}, \ldots, z_{p}\right)$ and $n \rightarrow\left(n_{1}, \ldots, n_{p}\right)$. Note that $G_{M, R, \alpha}(\Omega)$ is a vector space and a ring with respect to the arithmetic product of functions and it is closed under the standard rules of differentiation — see e.g. Hua and Rodino (1996). In particular, functions of Gevrey order $\alpha<1$ are entire and analytic in case $\alpha=1$ (Gevrey, 1918; Boas, 1954; Rudin, 1999). Further properties of the set $\boldsymbol{A}_{\alpha}(\Omega, \mathbb{E})$ of all functions $x \in \boldsymbol{H}(\Omega, \mathbb{E})$ having an asymptotic expansion $\hat{x}$ of order $\alpha$ can be e.g. found in Ramis (1993) or (Balser, 2000, p. 71f). In particular, if $\mathbb{E}$ is a Banach algebra, $\boldsymbol{A}_{\alpha}(\Omega, \mathbb{E})$ is a differential algebra with the map $J: \boldsymbol{A}_{\alpha}(\Omega, \mathbb{E}) \rightarrow \mathbb{E}[[z]]_{\alpha}$ being a homomorphism mapping products $x_{1} x_{2}$ to products $\left(J x_{1}\right)\left(J x_{2}\right)=\hat{x}_{1} \hat{x}_{2}$. As outlined in the previous section, it is desired that the map $J$ is injective in order to ensure that to every $\hat{x} \in \boldsymbol{A}_{\alpha}(\Omega, \mathbb{E})$ there exists a unique inverse image $x \in \boldsymbol{H}(\Omega, \mathbb{E})$. For the considered set $\boldsymbol{A}_{\alpha}(\Omega, \mathbb{E})$ of Gevrey asymptotics, the following two results (Malgrange, 1995; Balser, 2000) provide an answer to this problem.

Theorem 3.6 (Ritt's Theorem/Borel-Ritt Theorem). For $\alpha>1$, let $\hat{x}(z) \in \mathbb{C}[[z]]_{\alpha}$ and a sectorial region $\Omega$ of opening at most $(\alpha-1) \pi$ be arbitrarily given. Then there exists a function $x(z)$, holomorphic in $\Omega$, so that $x(z) \cong_{\alpha} \hat{x}(z)$ in $\Omega$.

This ensures that the map $J: \boldsymbol{A}_{\alpha}(\Omega, \mathbb{E}) \rightarrow \mathbb{E}[[z]]_{\alpha}$ is surjective in narrow regions if the opening of $\Omega$ is smaller than or equal to $(\alpha-1) \pi$.

Lemma 3.3 (Watson's Lemma). Suppose that $\Omega$ is a sectorial region of opening more than $(\alpha-1) \pi, \alpha>1$, and let $x \in \boldsymbol{H}(\Omega, \mathbb{E})$ satisfy $x \cong_{\alpha} \hat{0}$ in $\Omega$. Then $x(z) \equiv 0$ in $\Omega$.

This result ensures that the map $J: \boldsymbol{A}_{\alpha}(\Omega, \mathbb{E}) \rightarrow \mathbb{E}[[z]]_{\alpha}$ is injective for wider regions. On the other hand it can be shown that in this case surjectivity is lost (Malgrange, 1995; Balser, 2000).

The question now arises, how to uniquely determine the function $x(z)$ from a given formal power series $\hat{x}(z)$. From the results above, it follows that $\hat{x}(z)$ has to represent the Gevrey asymptotic expansion of a holomorphic function $x(z)$, i.e. $\hat{x} \in \boldsymbol{A}_{\alpha}(\Omega, \mathbb{E})$ in a sectorial region $\Omega$ of opening larger than $(\alpha-1) \pi$ due to Watson's Lemma. Once $x$ is found, it is natural to consider $x$ as the sum of $\hat{x}$ (Balser, 2000). Nevertheless, the determination of $x$ requires to find a functional which inverts the map $J: \boldsymbol{H}(\Omega, \mathbb{E}) \rightarrow \boldsymbol{A}_{\alpha}(\Omega, \mathbb{E})$. This leads to the introduction of summation methods.

\subsection{Summation of power series}

As the most common example of a summation method consider partial summation as the limit of the partial sum tends to infinity, i.e.

$$
\mathcal{S}_{P}\left(\sum_{n=0}^{\infty} \hat{x}_{n} z^{n}\right)=\lim _{N \rightarrow \infty} \sum_{n=0}^{N} \hat{x}_{n} z^{n} .
$$


Hence $\mathcal{S}_{P}$ can be interpreted as a linear functional mapping each convergent power series to its natural sum. Of course, this method is weak in the sense that it applies to convergent series only but provides insight into the structure of summation methods. Generalizing this approach, let $\mathcal{S}_{V}$ be a linear functional on a linear space $X_{\mathcal{S}}$ of sequences or equivalently series. Therefore introduce a sequence of functions $a_{n}(\xi)$ in the continuous parameter $\xi \geq 0$ and consider

$$
\mathcal{S}_{V}\left(\sum_{n=0}^{\infty} \hat{x}_{n} z^{n}\right)=\lim _{\xi \rightarrow \infty} \sum_{n=0}^{\infty} a_{n}(\xi) \hat{x}_{n} z^{n}=\lim _{\xi \rightarrow \infty} \hat{x}^{A}(z ; \xi) .
$$

If $\hat{x}^{A}(z ; \xi)$ converges for $|z|<\rho$, it defines a family of holomorphic functions in a disc $D=$ $D(0, \rho)$ of radius $\rho$ centered at the origin. Following Ramis (1993), the restriction to the case where convergence for $\xi \rightarrow \infty$ is locally uniform in $z$ (at least for $z$ in some sectorial region $\Omega$ of $D$ ) yields a holomorphic function $x(z)=\lim _{\xi \rightarrow \infty} x^{A}(z ; \xi)$ on $\Omega$. In this case, the formal power series $\hat{x}(z)$ is said to be $A$-summable on the region $\Omega$ and the function $x(z)=\left(\mathcal{S}_{V} \hat{x}\right)(z)$ is referred to as the $A$-sum of $\hat{x}(z)$. As a result, the space $X_{\mathcal{S}}$ consists of all $A$-summable series and is called the summability domain of the method $\mathcal{S}_{V}$. For a comprehensive discussion, the reader is refereed to Hardy (1949).

\subsubsection{General considerations}

In order to make a summation method suitable for the formal solution of $\mathrm{ODEs}^{3}$, the method has to satisfy further requirements (Ramis, 1993; Balser, 2000):

(i) The summability domain $X_{\mathcal{S}}$ has to be a differential algebra, prolonging the space $\mathbb{E}\{z\}$ of convergent power series $\hat{x}$ in $z \in \mathbb{C}$.

(ii) The functional $\mathcal{S}_{V}$ has to be regular, i.e. for any $\hat{x} \in \mathbb{E}\{z\}$ it follows that $\mathcal{S}_{P}(\hat{x})=\mathcal{S}_{V}(\hat{x})$.

(iii) The functional $\mathcal{S}_{V}$ has to be a linear homomorphism mapping products to products and derivatives to derivatives.

(iv) The operator $J: \boldsymbol{A}(\Omega, \mathbb{E}) \rightarrow \mathbb{E}[[z]]$ has to invert the functional $\mathcal{S}_{V}$. Note that this includes all operators $J: \boldsymbol{A}_{\alpha}(\Omega, \mathbb{E}) \rightarrow \mathbb{E}[[z]]_{\alpha}$.

Property (i) ensures first that differentiation is allowed and second that the summability domain enlarges the set of convergent series. Due to property (ii), the sum (the natural limit) of a convergent series is preserved under the functional $\mathcal{S}_{V}$. Assuming that (iii) is satisfied, it follows that given $\hat{x}(z), \hat{w}(z) \in X_{\mathcal{S}}, \mathcal{S}_{V}(\hat{x}(z) \circledast \hat{w}(z))=x(z) \cdot w(z)$ where $\cdot$ denotes multiplication in $\mathbb{E}$, and $\mathcal{S}_{V}(d \hat{x}(z) / d z)=d x(z) / d z$. Both properties are essential for the study of formal power series solutions to differential equations and are rarely satisfied for general summation processes. Property (iv) basically ensures, that given a formal power series $\hat{x}(z)$ satisfying a

\footnotetext{
${ }^{3}$ In order to deal with formal solutions to PDEs, further requirement such as uniform summability have to be introduced.
} 
given differential equation, its sum $\left.x(z)=\left(\mathcal{S}_{V} \hat{x}\right)(z)\right)$ can be uniquely determined in the region $\Omega$ and solves the differential equation with $x(z)$ being asymptotic to $\hat{x}(z)$ of order $\alpha$. Recall that due to Watson's Lemma 3.3, uniqueness requires that the sectorial region is of opening greater than $(\alpha-1) \pi$. One such summation method is the so-called $k$-summation, which was introduced by Ramis (1980) as a generalization of the work of Borel (1928) on the summation of divergent series.

\subsubsection{Summation in a direction}

$K$-summability can be considered as a generalization of the widely studied Borel summation (Borel, 1899; Watson, 1912; Borel, 1928; Hardy, 1949). The latter allows the summation of highly divergent power series by assigning to them Laplace-integrals, which if convergent, provide an analytic function in the argument $z \in \mathbb{C}$ of the power series.

In order to illustrate the principle setup, consider a convergent series $\hat{x}(z)=\sum_{n=0}^{\infty} \hat{x}_{n} z^{n} \in \mathbb{E}\{z\}$ with radius of convergence $\rho>0$. Let $z \in \mathbb{C}$ with $|z|<\rho$, such that there exists a holomorphic function $x(z)$ having $\hat{x}(z)$ as its power series expansion. Using standard arguments for uniformly convergent series, it can be verified that $x(z)$ admits an integral representation of the following form

$$
x(z)=\left(\mathcal{L}_{1} x_{B, 1}\right)(z)=\frac{1}{z} \int_{0}^{\infty} e^{-\frac{s}{z}} \underbrace{\sum_{n=0}^{\infty} \hat{x}_{n} \frac{s^{n}}{n !}}_{=: \hat{x}_{B, 1}(s)} d s=\sum_{n=0}^{\infty} \frac{\hat{x}_{n}}{z} \int_{0}^{\infty} e^{-\frac{s}{z}} \frac{s^{n}}{n !} d s=\sum_{n=0}^{\infty} \hat{x}_{n} z^{n},
$$

whereby the term

$$
\hat{x}_{B, 1}(z)=\left(\hat{\mathcal{B}}_{1} \hat{x}\right)(z)=\sum_{n=0}^{\infty} \hat{x}_{n} \frac{z^{n}}{n !}
$$

is called the formal Borel transform with $\hat{\mathcal{B}}_{1}$ the formal Borel operator (Ramis, 1980). Significantly, the integral in (3.17) still converges for divergent $\hat{x}(z)$ if further restrictions are imposed. Assume that $\hat{x}(z)$ is Gevrey of order $\alpha=2$, i.e. $\left\|\hat{x}_{n}\right\| \leq M / R^{n} n$ ! for $n \in \mathbb{N}_{0}$. Hence the formal Borel transform $\hat{x}_{B, 1}(z)$ converges in a neighborhood of the origin $z=0$ with say $|z|<\rho^{\prime}$, representing an analytic function $x_{B, 1}(z)=\left(\mathcal{S}_{P} \hat{x}_{B, 1}\right)(z)$ inside this open disc. Moreover, if there exits an analytic continuation of $x_{B, 1}(z)$ into a sector enclosing the positive real semi axis having exponential growth not more than one, i.e. $\exists c_{1}, c_{2}>0:\left\|x_{B, 1}\right\| \leq c_{1} \exp \left(c_{2}|z|\right)$, then the Laplace-integral (3.17) exists for $\Re z<1 / c_{2}$ (Hardy, 1949; Sokal, 1980; Ramis, 1993).

These considerations represent the basic idea of the so-called Borel summation Borel (1899, 1928), whereby detailed discussions on the necessary and sufficient conditions for Borel summability of divergent power series can be found in Watson (1912); Nevanlinna (1919); Sokal (1980).

Example 3.1 (Borel summation of the geometric series). In order to illustrate this approach, consider the geometric series in the complex plane $z \in \mathbb{C}$,

$$
\hat{x}(z)=\sum_{n=0}^{\infty} z^{n} .
$$


This example is due to Hardy (1949). Its formal Borel transform $\hat{x}_{B, 1}(z)=\sum_{n=0}^{\infty} z^{n} / n$ ! converges in the whole complex plane to the sum $x_{B, 1}(z)=\left(\mathcal{S}_{P} \hat{x}_{B, 1}\right)(z)=\exp (z)$ with obviously exponential growth at most one. Hence the Laplace-integral (3.17) exists for all $z \in \mathbb{C}: \Re z<1$.

A further generalization of Borel summation leads to $k$-summation (Ramis, 1980, 1993), which is able to sum certain divergent series whose coefficients grow faster than $M / R^{n} n$ !. Therefore, the Laplace-transform and Borel operators are extended to transforms and operators of a certain order to deal with formal power series $\hat{x}(z)=\sum_{n=0}^{\infty} \hat{x}_{n} z^{n}$ of Gevrey order $\alpha=1+1 / k$ for given $k>0$. Following Balser $(1994,2000)$, the Laplace-integral ${ }^{4}$ of order $k$ when applied to a holomorphic function $x \in \boldsymbol{A}^{(k)}(S, \mathbb{E})$ of exponential growth at most $k>0$ in a sector $S=S(d, \epsilon)$ yields

$$
\left(\mathcal{L}_{k} x\right)(z)=\frac{k}{z^{k}} \int_{0}^{\infty(\tau)} x(s) e^{-\left(\frac{s}{z}\right)^{k}} s^{k-1} d s .
$$

This integral converges absolutely and compactly with integration along $\arg s=\tau,|d-\tau| \leq \epsilon / 2$ in the open set $\cos (k[\tau-\arg z])>c(x, \tau)|z|^{k}$ with $c(x, \tau)$ sufficiently large. On the Riemann surface of the logarithm, this set consists of infinitely many connected components. Thereby it can be easily verified geometrically, that one component is given by the sectorial region $\Omega(\tau, \pi / k)$ of opening $\pi / k$ and bisecting direction $\tau$ defined by $k|d-\tau|<\pi / 2, \cos (k[\tau-\arg z])>c|z|^{k}$ - this region is depicted in Figure 3.1, right. Furthermore, it can be shown that $\left(\mathcal{L}_{k} x\right)(z)$ is independent of $\tau$ and holomorphic in the union of the corresponding regions $\Omega(\tau, \pi / k)$, i.e. a bounded sectorial region with opening $\epsilon+\pi / k$ and bisecting direction $d$ (Balser, 1994, 2000). Let

$$
\hat{x}_{B, k}(z)=\left(\hat{\mathcal{B}}_{k} \hat{x}\right)(z)=\sum_{n=0}^{\infty} \hat{x}_{n} \frac{z^{n}}{\Gamma\left(1+\frac{n}{k}\right)},
$$

denote the formal Borel operator of order $k$ of a given formal power series $\hat{x}(z)=\sum_{n=0}^{\infty} \hat{x}_{n} z^{n}$. Assume in addition that $\hat{x}_{B, k}(z)$ converges in a neighborhood of the origin $z=0$ and yields an analytical continuation into a sector $S=S(d, \epsilon)$ for some $\epsilon>0$ with exponential growth at most $k$ in $S$, then $x(z)=\left(\mathcal{L}_{k} \hat{x}_{B, k}\right)(z)$ denotes the $k$-sum of the series $\hat{x}(z)$. Under these conditions, it can be verified that

$$
\begin{aligned}
x(z)=\left(\mathcal{L}_{k} \hat{x}_{B, k}\right)(z) & =\frac{k}{z^{k}} \int_{0}^{\infty(\tau)} \sum_{n=0}^{\infty} \hat{x}_{n} \frac{s^{n}}{\Gamma\left(1+\frac{n}{k}\right)} s^{k-1} e^{-\left(\frac{s}{z}\right)^{k}} d s \\
& =\frac{k}{z^{k}} \sum_{n=0}^{\infty} \hat{x}_{n} \int_{0}^{\infty(\tau)} \frac{s^{n+k-1}}{\Gamma\left(1+\frac{n}{k}\right)} e^{-\left(\frac{s}{z}\right)^{k}} d s=\sum_{n=0}^{\infty} \hat{x}_{n} z^{n}
\end{aligned}
$$

whereby the latter equality requires a change of variables $\zeta=(s / z)^{k}$ and utilization of the definition of the $\Gamma$-function. The general result borrowed from Balser (2000), extends the

\footnotetext{
${ }^{4}$ Note that there exist several definitions of Laplace-transforms and Borel operators of a certain order throughout the available literature, whose consistency is not always obvious. Therefore, the notation of Balser (2000) is adopted in the sequel.
} 
results of Ramis $(1980,1993)$ to formal power series with coefficients in a fixed but arbitrary Banach space $\mathbb{E}$.

Definition 3.12 ( $k$-summability). Let $k>0, d \in \mathbb{R}$ and $\hat{x} \in \mathbb{E}[[z]]$ be given. The formal power series $\hat{x}$ is $k$-summable in direction $d$, if a sectorial region $\Omega=\Omega(d, \varsigma)$ of opening $\varsigma>\pi / k$ and a function $x \in \boldsymbol{A}_{1+1 / k}(\Omega, \mathbb{E})$ exist with $J: \boldsymbol{A}_{1+1 / k}(\Omega, \mathbb{E}) \rightarrow \mathbb{E}[[z]]_{1+1 / k}$ such that $J(x)=\hat{x}$.

Hence, $\hat{x} \in \mathbb{E}[[z]]_{1+1 / k}$ and $x \in \boldsymbol{A}_{1+1 / k}(\Omega, \mathbb{E})$ is unique by Watson's Lemma 3.3. As a result, if $\hat{x}_{B, k}=\mathcal{B}_{k} \hat{x}$ converges and if $x_{B, k}=\mathcal{S}_{P} \hat{x}_{B, k}$ is holomorphic and of exponential growth at most $k$ in a sector $S(d, \epsilon)$ for some $\epsilon>0$, then $\hat{x}$ is $k$-summable in direction $d$ with the function $x=\mathcal{S}_{k, d} \hat{x}=\mathcal{L}_{k} \circ \mathcal{S}_{p} \circ \mathcal{B}_{k} \hat{x}$ representing the $k$-sum in direction $d$. In particular for $k=1$, the results for Borel summation are recovered, such that 1-summability is equivalent to Borel summability.

The computation of the $k$-sum of a given formal power series $\hat{x}$ of Gevrey order $\alpha=1+1 / k$, i.e. $\hat{x} \in \mathbb{E}[[z]]_{1+1 / k}$ requires to verify that

(i) the formal Borel transform $\hat{x}_{B, k}(z)=\left(\mathcal{B}_{k} \hat{x}\right)(z)$ converges for some $|z|<\rho$, such that $x_{B, k}=\mathcal{S}_{P} \hat{x}_{B, k}$ and that

(ii) the function $x_{B, k}$ can be analytically continued into a sector $S(d, \epsilon)$ of infinite opening for some $\epsilon>0$ with exponential growth at most $k$ within the sector $S(d, \epsilon)$.

In this case, inversion of the Borel transform using the Laplace transform $\mathcal{L}_{k}$ of order $k$ determines the $k$-sum $x=\mathcal{L}_{k} \hat{x}_{B, k}$ of the formal power series $\hat{x}(z)$. Note that the integral evaluation can be performed numerically using e.g. quadrature rules.

As an example, the $k$-sum of the Euler series, which is divergent for any $z \in \mathbb{C}$ if $|z| \neq 0$ is determined:

Example 3.2 ( $\boldsymbol{k}$-summability of the Euler series). Consider first the application of the formal Borel operator of order $k$ (3.21) on the series $\hat{x}(z)=\sum_{n=0}^{\infty} \Gamma\left(1+\frac{n}{k}\right) z^{n}$ with $z \in \mathbb{C}$, i.e.

$$
\hat{x}_{B, k}(z)=\left(\hat{\mathcal{B}}_{k} \hat{x}\right)(z)=\sum_{n=0}^{\infty} z^{n} .
$$

Since $\left(\mathcal{S}_{P} \hat{x}_{B, k}\right)(z)=1 /(1-z)$, the formal Borel operator of order $k$ can be analytically continued into any sector excluding the positive real axis with exponential growth obviously less than $k$. As a result, the series $\hat{x}(z)$ is $k$-summable in any direction $d \neq 2 j \pi, j \in \mathbb{Z}$. Hence let $k=1$ and replace $z$ by $-z$ such that $\hat{x}(z)$ corresponds to the Euler series. Since now $\left(\mathcal{S} \hat{x}_{B, k}\right)(z)=1 /(1+z)$, it follows similarly that the Euler series is 1 -summable in any direction $d \neq(2 j+1) \pi, j \in \mathbb{Z}$. In particular,

$$
x(z)=\left(\mathcal{L}_{1} \hat{x}_{B, 1}\right)=\frac{1}{z} \int_{0}^{\infty(\tau)} \frac{1}{1+s} e^{-\frac{s}{z}} d s=\frac{e^{\frac{1}{z}}}{z} \int_{0}^{z} \frac{e^{-\frac{1}{\zeta}}}{\zeta} d \zeta,
$$

which can be obtained by a change of variables $\zeta=z /(1+s)$. Thereby $k$-summability for $k>1$ is implied by the following Lemma. 
Lemma 3.4 ((Balser, 2000)). Let $\hat{x}(z)$ be $k_{1}$-summable in direction $d$, let $k>k_{1}$ and define $k_{2}$ by $1 / k_{2}=1 / k_{1}-1 / k$. Then $\hat{x}_{B, k}=\hat{\mathcal{B}}_{k} \hat{x}$ is $k_{2}$-summable in direction $d$.

Further properties of $k$-summability can be found in Ramis (1993), (Balser, 1994, p. 13f), and (Balser, 2000, p. 100f), whereby in particular regularity of the $k$-summation can be proven. However, the algebraic properties of the set $\mathbb{E}\{z\}_{k, d}$ of $k$-summable power series in direction $d$ are remarkable and therefore briefly summarized below. It can be proven that $\mathbb{E}\{z\}_{k, d}$ has the same algebraic properties as the space $\mathbb{E}\{z\}$ of convergent power series (Balser, 2000, p. 103) as is summarized below.

Theorem 3.7. For fixed, but arbitrary $k>0$ and $d$, let $\hat{x}, \hat{v}, \hat{w} \in \mathbb{E}\{z\}_{k, d}$ be given. Then

$$
\begin{array}{rlrl}
\hat{v}+\hat{w} & \in \mathbb{E}\{z\}_{k, d}, & & \mathcal{S}_{k, d}(\hat{v}+\hat{w})=\mathcal{S}_{k, d} \hat{v}+\mathcal{S}_{k, d} \hat{w} \\
\hat{x}^{\prime} & \in \mathbb{E}\{z\}_{k, d}, & & \mathcal{S}_{k, d}\left(\hat{x}^{\prime}\right)=\frac{d}{d z}\left(\mathcal{S}_{k, d} \hat{x}\right) \\
\int_{0}^{z} \hat{x}(s) d s & \in \mathbb{E}\{z\}_{k, d}, & \mathcal{S}_{k, d}\left(\int_{0}^{z} \hat{x}(s) d s\right)=\int_{0}^{z}\left(\mathcal{S}_{k, d} \hat{x}\right)(s) d s .
\end{array}
$$

If $\mathbb{E}$ is a Banach algebra, then in addition

$$
\hat{v} \hat{w} \in \mathbb{E}\{z\}_{k, d}, \quad \mathcal{S}_{k, d}(\hat{v} \hat{w})=\left(\mathcal{S}_{k, d} \hat{v}\right)\left(\mathcal{S}_{k, d} \hat{w}\right) .
$$

Hence, the well-known properties of convergent power series directly carry over to $k$-summable power series, such that computations can be performed in a similar way.

As outlined throughout this section and the presented examples, the theoretical verification of $k$-summability of a given formal power series is in general a challenging task. For formal power series solutions to general meromorphic nonlinear ODEs, quite general results are provided e.g. in Balser $(1994,2000)$. Thereby, it has to be pointed out, that $k$-summability is still too weak to sum all the arising series solutions, which led to the introduction of so-called multisummability methods (see e.g. Malgrange (1995); Ramis (1993); Balser (1994, 2000) and the references therein). It can be shown that every multi-summable formal power series can be written as a sum of finitely many formal power series, each of which being $k$-summable in some direction for an individual value of $k$ depending on the series.

Nevertheless, in many situations it is only possible to show that there exists indeed a power series solution to a given problem, whereby only a finite and usually limited number of series coefficients is (analytically and numerically) accessible. This fact leads to the introduction of socalled generalized sequence transformations, which act on a finite number of series coefficients in order to approximate or recover the complete power series solution of a given problem. Within this framework, a modification of $k$-summability $\mathcal{S}_{k, d}$ is proposed in Section 3.4, namely the $(N, \xi)$-approximate $k$-sum, which approximately retains the powerful properties of $\mathcal{S}_{k, d}$ and accounts for the described problems. 


\subsection{Towards numerical implementation and convergence acceleration: the $(\mathbf{N}, \xi)$-approximate $\mathbf{k}-$ sum}

As illustrated in the previous section, $k$-summability is a powerful tool for the summation of certain highly divergent series. On the other hand for computational purposes and practical problems, typically only a finite number of series coefficients is processable if at all available. In order to account for this fact, a modification to $k$-summation, namely the so-called $(N, \xi)-$ approximate $k$-sum is proposed in Wagner (2003), Meurer and Zeitz (2004a), and Wagner et al. (2004), which allows to accelerate convergence of a given series and to sum certain divergent series using only a finite number $N$ of series coefficients.

In order to motivate this approach, the previously introduced transformations have to be rewritten in a slightly different manner to illustrate their effect on the coefficients of a given sequence. For the sake of simplicity consider first the 1-sum or Borel sum of a convergent power series $\hat{x}(z)=\sum_{n=0}^{\infty} \hat{x}_{n} z^{n}$ with radius of convergence $\rho>0$. Let $s_{n}(z)$ denote its $n^{-}$ th partial sum $s_{n}(z)=\sum_{j=0}^{n} \hat{x}_{j} z^{j}$. Then it follows from (3.17) after a change of coordinates $\xi=s / z$ and integration by parts that

$$
\begin{aligned}
\mathcal{L}_{1}\left(\hat{x}_{B, 1}\right)(z) & =\frac{1}{z} \int_{0}^{\infty} e^{-s / z} \sum_{n=0}^{\infty} \hat{x}_{n} \frac{s^{n}}{n !} d s \stackrel{\xi=s / z}{=} \int_{0}^{\infty} e^{-\xi} \sum_{n=0}^{\infty} \hat{x}_{n} z^{n} \frac{\xi^{n}}{n !} d \xi \\
& \stackrel{\text { p. int. }}{=}\left[-e^{-\xi} \sum_{n=0}^{\infty} \hat{x}_{n} z^{n} \frac{\xi^{n}}{n !}\right]_{0}^{\infty}+\int_{0}^{\infty} e^{-\xi} \sum_{n=0}^{\infty} \hat{x}_{n+1} z^{n+1} \frac{\xi^{n}}{n !} d \xi \\
& =\hat{x}_{0}+\int_{0}^{\infty} e^{-\xi} \sum_{n=0}^{\infty}\left[s_{n+1}(z)-s_{n}(z)\right] \frac{\xi^{n}}{n !} d \xi \\
& =\hat{x}_{0}+\int_{0}^{\infty} \frac{d}{d \xi}\left[e^{-\xi} \sum_{n=0}^{\infty} \frac{s_{n}(z) \xi^{n}}{n !}\right] d \xi=\hat{x}_{0}+\int_{0}^{\infty} \frac{d}{d \xi} \mathcal{B}(z, \xi) d \xi \\
& =\lim _{\xi \rightarrow \infty} \mathcal{B}(z, \xi) .
\end{aligned}
$$

As a result, the sum $x(z)$ of the convergent power series $\hat{x}(z)$ can be equivalently determined by

$$
x(z)=\mathcal{S}_{B} \hat{x}(z):=\lim _{\xi \rightarrow \infty} e^{-\xi} \sum_{n=0}^{\infty} \frac{s_{n}(z) \xi^{n}}{n !}=\lim _{\xi \rightarrow \infty} \mathcal{B}(z, \xi),
$$

where $s_{n}(z)=\sum_{j=0}^{n} \hat{x}_{n} z^{n}$ and $\mathcal{S}_{B}$ denotes the Borel sum (Borel, 1928; Hardy, 1949). The Borel summation procedure (3.23) is an element of the methods defined by means of integral functions (Hardy, 1949): Let $P(\xi)=\sum_{n} p_{n} \xi^{n}$ define an entire function, i.e. $P(\xi)$ converges for all $\xi \in \mathbb{C}$ with non-negative coefficients $p_{n}$. If

$$
\left(\mathcal{S}_{V} \hat{x}\right)(z)=\lim _{\xi \rightarrow \infty} \frac{\sum_{n} p_{n} s_{n}(z) \xi^{n}}{\sum_{n} p_{n} \xi^{n}} \rightarrow x(z)
$$


converges for $\xi \rightarrow \infty$, its limit $x(z)$ is called the sum of the series $\hat{x}(z)$. For $p_{n}=1 / n$ !, the Borel sum is recovered from (3.24). On the other hand by choosing $p_{n}=1 / \Gamma(1+n / k), k$-summation can be similarly re-defined as a subset of the means of integral functions approach, i.e.

$$
x(z) \cong \hat{x}(z)=\lim _{\xi \rightarrow \infty} \frac{\sum_{n=0}^{\infty} s_{n}(z) \frac{\xi^{n}}{\Gamma\left(1+\frac{n}{k}\right)}}{\sum_{n=0}^{\infty} \frac{\xi^{n}}{\Gamma\left(1+\frac{n}{k}\right)}}=:\left(\mathcal{S}_{B}^{k} \hat{x}\right)(z),
$$

as similarly outlined in Balser and Braun (2000). In particular, the denominator function of the fractional term is also known as the Mittag-Leffler function of index $1 / k$ i.e. $E_{1 / k}(\xi)=$ $\sum_{n=0}^{\infty} \xi^{n} / \Gamma(1+n / k)$, which is entire for $k>0$ (Mittag-Leffler, 1903; Miller, 1993). Note that $E_{1 / k}(\xi)$ is a direct generalization of an exponential functions since $E_{1}(\xi)=\exp \xi$. As a simple example for the application of $\mathcal{S}_{B}^{k}$, consider again the geometric series.

Example 3.3 (Summation of the geometric series by $\mathcal{S}_{B}^{k}$ ). It can be easily verified that the $n$-th partial sum $s_{n}(z)$ of the geometric series $\hat{x}(z)=\sum_{n=0}^{\infty} z^{n}$ is given by $s_{n}(z)=\left(z^{n+1}-\right.$ $1) /(z-1)$. Hence it follows that

$$
\left(\mathcal{S}_{B}^{k} \hat{x}\right)(z)=\lim _{\xi \rightarrow \infty} E_{1 / k}^{-1}(\xi) \sum_{n=0}^{\infty} \frac{z^{n+1}-1}{z-1} \frac{\xi^{n}}{\Gamma\left(1+\frac{n}{k}\right)}=\frac{1}{1-z}-\frac{z}{1-z} \lim _{\xi \rightarrow \infty} \frac{E_{1 / k}(z \xi)}{E_{1 / k}(\xi)}=\frac{1}{1-z}=x(z)
$$

provided that $\Re z<1$. As expected from Example 3.1, the method $\mathcal{S}_{B}^{k}$ also allows to sum the geometric series in the whole complex half plane $\Re z<1$.

From an implementation point of view, the explicit determination of the limits in either Borel summation (3.23) or $k$-summation (3.25) requires knowledge of the complete sequence $\left\{\hat{x}_{n}\right\}_{n=0}^{\infty}$. Nevertheless in many applications, only a finite number $N$ of series coefficients is available if computable at all. Evaluation of (3.25) with a maximum of $N<\infty$ coefficients $\left\{\hat{x}_{n}\right\}_{n=0}^{N-1}$ yields a value of zero for the sum in the limit as $\xi \rightarrow \infty$, since $\sum_{n=0}^{\infty} \xi^{n} / \Gamma(1+n / k) \approx A \exp \left(B \xi^{1 / k}\right)$ with constants $A, B$ depending on $k$. Hence, if only a finite number of series coefficients is available, the limiting process has to be replaced which results in the so-called $(N, \xi)$-approximate $k^{-}$ summation.

\subsubsection{The $(\mathbf{N}, \xi)$-approximate $\mathrm{k}$-sum}

If only a finite number of series coefficients is computable, Wagner (2003), Meurer and Zeitz (2004a), and Wagner et al. (2004) introduce the $(N, \xi)$-approximate $k$-summation as a variant of $k$-summation within the methods of means of integral functions:

Definition $3.13\left((\boldsymbol{N}, \xi)\right.$-approximate $\boldsymbol{k}$-sum). The $(N, \xi)$-approximate $k$-sum $\mathcal{S}_{k}^{N, \xi}$ of a $k$-summable formal power series $\hat{x}(z)=\sum_{n=0}^{\infty} \hat{x}_{n} z^{n}$ is defined as

$$
\mathcal{S}_{k}^{N, \xi} \hat{x}(z)=\frac{\sum_{n=0}^{N} s_{n}(z) \frac{\xi^{n}}{\Gamma\left(1+\frac{n}{k}\right)}}{\sum_{n=0}^{N} \frac{\xi^{n}}{\Gamma\left(1+\frac{n}{k}\right)}}
$$

where $s_{n}(z)=\sum_{j=0}^{n} \hat{x}_{j} z^{j}$ denotes the $n$-th partial sum. 
Note that this definition also fits into the terminology of generalized sequence transformations, which are briefly introduced in Section 3.5. In order to illustrate the underlying idea and the properties of the proposed summation approach, consider the $(N, \xi)$-approximate $k$-sum of the geometric series.

Example 3.4 $((\boldsymbol{N}, \xi)$-approximate 1-sum of the geometric series). It is shown in Example 3.1, that the geometric series $\hat{x}(z)=\sum_{n=0}^{\infty} z^{n}$ is $k$-summable outside the unit disc for every $z \in \mathbb{C}$ with $|z|<1$ to the classical limit $x(z)=1 /(1-z)$. Application of $\mathcal{S}_{1}^{N, \xi}$ yields that

$$
\left(\mathcal{S}_{1}^{N, \xi} \hat{x}\right)(z)=\frac{1}{1-z}-\frac{z}{1-z} \frac{\sum_{n=0}^{N} \frac{(z \xi)^{n}}{n !}}{\sum_{n=0}^{N} \frac{\xi^{n}}{n !}}=x(z)+e(z, \xi, n) .
$$

Hence, the Borel sum is recovered with an error $e(z, \xi, N)$ depending on $z$ and on the choice of $\xi$ and $N$. However, a suitable choice of the summation parameters $\xi$ and $N$ minimizes the error e $(z, \xi, N)$. Since for general series, typically the number $N$ of available series coefficients is limited, consider $\partial e(z, \xi, N) / \partial \xi=0$ for fixed $z$ and $N$ and solve for $\xi$, i.e.

$$
\frac{\partial e(z, \xi, N)}{\partial \xi}=-\frac{z}{1-z} \frac{z \sum_{n=0}^{N-1} \frac{(z \xi)^{n}}{n !} \sum_{n=0}^{N} \frac{\xi^{n}}{n !}-\sum_{n=0}^{N} \frac{(z \xi)^{n}}{n !} \sum_{n=0}^{N-1} \frac{\xi^{n}}{n !}}{\left(\sum_{n=0}^{N} \frac{\xi^{n}}{n !}\right)^{2}}=: \frac{e^{(n)}(z, \xi, N)}{e^{(d)}(z, \xi, N)} \stackrel{!}{=} 0 .
$$

Due to a cancellation of the terms of order $2 N-1$ in $\xi$, the numerator polynomial $e^{(n)}(z, \xi, N)$ is of order $2 N-2$. For the sake of simplicity let $z \in \mathbb{R}$. Hence it is natural to restrict $\xi$ to the positive real axis in order to ensure that the result $\mathcal{S}_{1}^{N, \xi} \hat{x}$ is in $\mathbb{R}$. Numerical results indicate that for given $z<1$, the real roots of $e^{(n)}(z, \xi, N)$ provide a minimal error $e(z, \xi, N)$ whenever $N$ is even. Table 3.1 summarizes the results for $z=-2$ and $N \in\{4,10,20,50\}$. There, the given value of $\xi$ represents the real root $\xi$ of the numerator polynomial $e^{(n)}(z, \xi, N)=0$ for which the minimal error is obtained.

Table 3.1: Error induced by $(N, \xi)$-approximate 1-sum applied to the geometric series of Example 3.4 for $z=-2$.

\begin{tabular}{l|l|l|l}
\hline$N$ & Root $\xi$ & Error $e(-2, \xi, N)$ & $\left(\mathcal{S}_{k}^{N, \xi} \hat{x}\right)(z=-2)$ \\
\hline \hline 4 & 0.89268837 & 0.0775168 & 0.4108501 \\
\hline 10 & 1.7421111 & 0.005656 & 0.338990 \\
\hline 20 & 3.15724 & 0.00007893 & 0.333412 \\
\hline 50 & 7.37305 & $2.49224 \times 10^{-10}$ & 0.333333 \\
\hline \multicolumn{2}{l}{ Borel sum $x(z)=1 /(1-z)$} & $\frac{1}{3}$ \\
\hline
\end{tabular}

Further properties of the $(N, \xi)$-approximate $k$-sum are summarized below:

Proposition 3.1. Given a $k$-summable series $\hat{x}(z) \in \boldsymbol{A}_{1 / k}(S, \mathbb{E})$, then 
(i) For every $\epsilon_{1}>0$, there exists $\xi_{0}>0$ such that

$$
\left\|\lim _{\xi \rightarrow \infty} \frac{\sum_{n=0}^{\infty} s_{n}(z) \frac{\xi^{n}}{\Gamma\left(1+\frac{n}{k}\right)}}{\sum_{n=0}^{\infty} \frac{\xi^{n}}{\Gamma\left(1+\frac{n}{k}\right)}}-\frac{\sum_{n=0}^{\infty} s_{n}(z) \frac{\xi_{0}^{n}}{\Gamma\left(1+\frac{n}{k}\right)}}{\sum_{n=0}^{\infty} \frac{\xi_{0}^{n}}{\Gamma\left(1+\frac{n}{k}\right)}}\right\|<\epsilon_{1} .
$$

(ii) For every $\epsilon_{2}>0$ and $\xi>0$, there exists an integer $N(\xi)$ such that

$$
\left\|\frac{\sum_{n=0}^{\infty} s_{n}(z) \frac{\xi^{n}}{\Gamma\left(1+\frac{n}{k}\right)}}{\sum_{n=0}^{\infty} \frac{\xi^{n}}{\Gamma\left(1+\frac{n}{k}\right)}}-\frac{\sum_{n=0}^{N} s_{n}(z) \frac{\xi^{n}}{\Gamma\left(1+\frac{n}{k}\right)}}{\sum_{n=0}^{N} \frac{\xi^{n}}{\Gamma\left(1+\frac{n}{k}\right)}}\right\|<\epsilon_{2} .
$$

Hence, the difference between the $k$-sum and the $(N, \xi)$-approximate $k$-sum of a given series is not greater than $\epsilon=\epsilon_{1}+\epsilon_{2}$.

The proof directly follows from the assumption of $k$-summability of the formal series (Wagner, 2003).

\subsubsection{Numerical example: the divergent Euler series}

In the following, the $(N, \xi)$-approximate $k$-summation (3.26) is applied to approximate the sum of the Euler series $\hat{x}(z)=\sum_{n=0}^{\infty} n !(-z)^{n}$, which is studied in Example 3.2 in the context of $k$-summability. Based on this example, it is additionally desired to motivate a simple approach for the determination of the summation parameters $\xi$ and $k$ in $\mathcal{S}_{k}^{N, \xi}$.

Therefore consider Figure 3.2, where sections of the numerical results for the application of $\mathcal{S}_{k}^{N, \xi}$ to the Euler series with $z=1$ are depicted for fixed $k=0.8$ and $N, \xi$ varied, as well as fixed $N=30$ with $\xi, k$ varied. The results illustrate the effect of omitting the evaluation of the two limits in the original $k$-summation process, i.e. $N \rightarrow \infty$ and $\xi \rightarrow \infty$. As can be clearly deduced from Figure 3.2 (left), it is necessary to determine a suitable path in the $(N, \xi)$-domain to obtain a sufficient approximation of the sum of series, which is obtained along the constant plane. On the other hand and in view of the application to formal solutions to DPS, it is often required to determine the sum of a possibly divergent series without the ability to vary $N$ over a large interval but rather to restrict $N$ to a finite number which depends on the complexity of the differential recursion defining the series coefficients. Numerical results for this case are shown in Figure 3.2 (right), which confirm the existence of parameter combinations $(\xi, k)$ for fixed $N$, providing accurate results for approximation of the sum of the series - here along the evolving marked plane. This behavior is typical for the introduced approach when approximating the $k$-sum of a given series by its $(N, \xi)$-approximate $k$-sum.

Based on these observations, two simple algorithms for the determination of suitable summation parameters can be formulated:

(A1) If $N$ can be varied in a large interval, a gradient search over $N$ can be performed to determine the minimal gradient of the curve $\mathcal{S}_{\breve{k}}^{N, \check{\xi}}(\hat{x})$ for fixed $\check{\xi}, \check{k}>0$. 

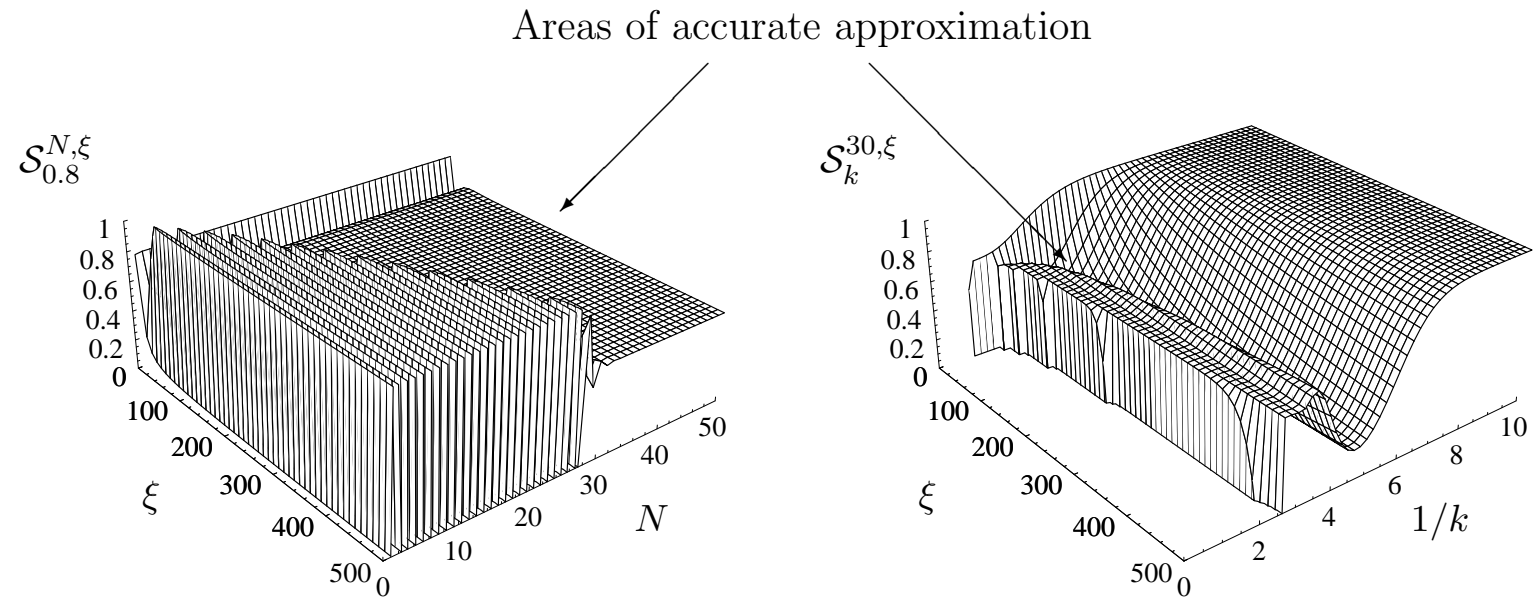

Figure 3.2: Numerical results for the application of $\mathcal{S}_{k}^{N, \xi}$ to the Euler series with $z=1$ for fixed $k=0.8$ with $N, \xi$ varied (left) and fixed $N=30$ with $\xi, k$ varied (right).

(A2) If $N$ is fixed, many problems allow to consider the quotient of $q_{\mathcal{S}}:=\mathcal{S}_{k}^{N, \xi}(\hat{x}) / \mathcal{S}_{k+\epsilon}^{N, \xi}(\hat{x})$ for fixed $N$ and $k$ over $\xi$. Whenever the marked area in Figure 3.2 (right) is reached, $q_{\mathcal{S}} \approx$ const. and exhibits oscillating behavior outside. Hence, determination of minimal gradient $d q_{\mathcal{S}} / d \xi$ over $\xi$ provides the desired approximation.

Although, these simple rules often provide a highly accurate approximation, certain knowledge of the series under consideration is required for the choice of suitable for $\xi$ and $k$ for (A1) or $N, k$, and $\epsilon$ for $(A 2)$. Numerical results for the applications of these ideas to approximate the sum of the divergent Euler series, are summarized in Table 3.2. It can be observed that the sum of the series determined from (3.7) can be approximated up to 6 decimal places.

Note that in view of the application of the $(N, \xi)$-approximate $k$-summation to feedforward control design, an optimization based approach for the determination of the summation parameters is proposed in Section 4.4.1.2. In the following, the approximation of the sum of divergent Euler series will serve a benchmark example to evaluate different summation techniques. These are introduced within the framework of generalized sequence transformations (Brezinski, 1977).

Table 3.2: Approximation of the sum of the Euler series (3.7) with $z=1$ using the $(N, \xi)$-approximate $k$-summation. The quantities determined from (A1) and (A2), respectively, are marked bold-faced.

\begin{tabular}{l|l|l|l|l|l}
\hline & $N$ & $\xi$ & $k$ & $\mathcal{S}_{k}^{N, \xi} \hat{x}$ & Accuracy [Decimals] \\
\hline \hline (A1) & $\mathbf{8 6}$ & 100 & 2 & 0.59634767397679 & 6 \\
\hline (A2) & 70 & $\mathbf{1 0 4}$ & 2.1 & 0.59634780283721 & 6 \\
\hline \multicolumn{6}{l}{ Sum from (3.7) } \\
\hline
\end{tabular}




\subsection{General sequence transformations}

Sequence transformations are algorithmic tools to overcome convergence problems of general sequences such as slow convergence or divergence. In the following, only the basic ideas are presented, and the interested reader is referred to the comprehensive treatise in Brezinski (1977); Weniger $(1989,1994)$ and the references therein.

\subsubsection{General considerations}

Differing from the previous sections studying summability methods, sequence transformations in general act on a finite number of series or sequence coefficients, whereby no algebraic structure can be identified with the domain of transformable sequences. For example, in Section 3.3.2 it is illustrated that the space $\mathbb{E}\{z\}_{k, d}$ of $k$-summable power series is a differential algebra in case $\mathbb{E}$ is a Banach algebra. This in particular allows to work with $k$-summable power series in the same manner as is well-known from convergent power series. Sequence transformations on the other hand, represent an algorithmic framework with the goal to compute the limit or the sum of a given (divergent) series considering just a finite number of series coefficient by purely algebraic operations without evaluation of limiting processes.

Let $\mathcal{T}_{p}$ denote a generalized summation process which assigns to the $(p+1)$ partial sums $s_{n}(z), \ldots, s_{n+p}$ with $s_{n}(z)=\sum_{j=0}^{n} \hat{x}_{j} z^{j}$ an element $s_{n}^{\mathcal{T}}(z)$ of the transformed sequence $\left\{s_{n}^{\mathcal{T}}(z)\right\}_{n=0}^{\infty}$, i.e.

$$
s_{n}^{\mathcal{T}}(z)=\mathcal{T}_{p}\left(s_{n}(z), s_{n+1}(z), \ldots, s_{n+p}(z)\right), \quad p \in \mathbb{N}_{0} .
$$

Convergency is improved if

$$
\lim _{n \rightarrow \infty} \frac{s_{n}^{\mathcal{T}}(z)-x(z)}{s_{n}(z)-x(z)}=0
$$

where $x(z)=s_{\infty}(z)$ is the limit of the untransformed sequence $\left\{s_{n}(z)\right\}_{n=0}^{\infty}$ in a neighborhood of the origin (Weniger, 1994). This of course requires convergency (possibly slow) of $\lim _{n \rightarrow \infty} s_{n}(z)=\mathcal{S}_{P}\left(\sum_{n=0}^{\infty} \hat{x}_{n} z^{n}\right)$ for some $z$ with $|z|<\rho$.

Furthermore, linearity as well as regularity can be defined in the standard way. A prototype example of a generalized summation process being linear and regular is given by so-called regular matrix transformations (Weniger, 1994), i.e. the transformed sequence $\left\{s_{n}^{\mathcal{T}}\right\}_{n=0}^{\infty}$ is determined from the original sequence $\left\{s_{n}\right\}_{n=0}^{\infty}$ of partial sums by introducing weighted mean values

$$
s_{n}^{\mathcal{T}}(z)=\sum_{p=0}^{n} \mu_{n, p} s_{p}(z) .
$$

Note that it is possible to provide necessary and sufficient conditions for the weights $\mu_{n, p}$ to ensure regularity of the method (Hardy, 1949; Knopp, 1964). 
On the other hand as outlined by Weniger $(1989,1994)$, nonlinear and possibly non-regular sequence transformations increase the power of the method with the drawback of possibly shrinking the domain of applicability. Nevertheless, such a approaches are appealing in both theory and application, which is reflected by an immense amount of literature - see e.g. the discussion in (Weniger, 1994, p. 21f).

The construction of GSTs is usually based on the decomposition of a convergent or divergent sequence $\left\{s_{n}(z)\right\}_{n=0}^{\infty}$ into a limit (if $\lim _{n \rightarrow \infty} s_{n}(z)=x(z)$ exists for $|z|<\rho$ ) or an antilimit $x(z)$, and a remainder $R(z, n)$

$$
s_{n}(z)=x(z)+R(z, n)
$$

for $n \in \mathbb{N}_{0}$. In order to determine $x(z)$ for a given sequence $\left\{s_{n}(z)\right\}_{n=0}^{\infty}$, the question arises of how to construct approximations to the remainders $R(z, n)$. By applying a generalized summation process $\mathcal{T}$, it is desired to generate transformed sequences $\left\{s_{n}^{\mathcal{T}}(z)\right\}_{n=0}^{\infty},\left\{R^{\mathcal{T}}(z, n)\right\}_{n=0}^{\infty}$, which can be again decomposed for any $n \in \mathbb{N}_{0}$ into

$$
s_{n}^{\mathcal{T}}(z)=x(z)+R^{\mathcal{T}}(z, n) .
$$

Obviously, convergence acceleration is obtained if the new sequence of transformed remainders $\left\{R^{\mathcal{T}}(z, n)\right\}_{n=0}^{\infty}$ vanish faster than the original sequence $\{R(z, n)\}_{n=0}^{\infty}$. Furthermore, it is possible to sum divergent series by the generalized summation process $\mathcal{T}$, if the sequence of transformed remainders $\left\{R^{\mathcal{T}}(z, n)\right\}_{n=0}^{\infty}$ vanishes for $n \rightarrow \infty$ (Weniger, 1989). For the explicit construction, several approaches exist, which are usually based on iterated processes, estimates on the remainder terms or Padé approximations.

Remark 3.3 (Connections between $(\boldsymbol{N}, \xi)$-approximate $\boldsymbol{k}$-summation and GSTs). In view of Eqn. (3.27), the introduced $(N, \xi)$-approximate $k$-summation (3.26) can be interpreted within the framework of GSTs, since

$$
\left(\mathcal{S}_{k}^{N, \xi} \hat{x}\right)(z)=\frac{\sum_{n=0}^{N} s_{n}(z) \frac{\xi^{n}}{\Gamma\left(1+\frac{n}{k}\right)}}{\sum_{n=0}^{N} \frac{\xi^{n}}{\Gamma\left(1+\frac{n}{k}\right)}}=\mathcal{T}_{N}^{\xi, k}\left(s_{0}(z), s_{1}(z), \ldots, s_{N}(z)\right) .
$$

It is easy to see that $\mathcal{T}_{N}^{\xi, k}$ is a linear sequence transformation due to

$$
\begin{aligned}
\mathcal{T}_{N}^{\xi, k}\left(a s_{0}(z), a s_{1}(z), \ldots, a s_{N}(z)\right) & =a \mathcal{T}_{N}^{\xi, k}\left(s_{0}(z), s_{1}(z), \ldots, s_{N}(z)\right) \\
\mathcal{T}_{N}^{\xi, k}\left(b+s_{0}(z), b+s_{1}(z), \ldots, b+s_{N}(z)\right) & =b+\mathcal{T}_{N}^{\xi, k}\left(s_{0}(z), s_{1}(z), \ldots, s_{N}(z)\right)
\end{aligned}
$$

for any $a, b$. Furthermore following the ideas of Weniger (1994), it can be verified that $\mathcal{T}_{N}^{\xi, k}$ represents a regular GST summing every convergent series to its natural sum.

\subsubsection{Examples of linear and nonlinear sequence transformations}

In view of a comparison of GST with $(N, \xi)$-approximate $k$-summation for convergence acceleration and summation of divergent series, several widely studied algorithms are summarized 
below. These are either classical iterated schemes (Aitken's $\Delta^{2}$-formula), classical algorithms with explicit remainder estimates (Levin $U$-transformation), and modified versions of the latter approach (Weniger's $\delta$-algorithm). Each method is formulated to accelerate convergence of a formal series $\hat{x}=\sum_{n} \hat{x}_{n} z^{n}$. A detailed study of their properties can be found e.g. in Brezinski (1977); Weniger $(1989,1994)$ and the literature cited therein.

- Aitken's $\Delta^{2}$-formula

$$
\begin{aligned}
& \mathcal{A I}_{0}^{\left(q_{\mathcal{A I}}\right)}(z)=s_{q_{\mathcal{A I}}}(z) \\
& \mathcal{A I}_{p_{\mathcal{A I}}}^{\left(q_{\mathcal{A} \mathcal{I}}\right)}(z)=\mathcal{A I}_{p_{\mathcal{A I}}-1}^{\left(q_{\mathcal{A}}\right)}(z)-\frac{\left[\Delta \mathcal{A I}_{p_{\mathcal{A I}}-1}^{\left(q_{\mathcal{I}}\right)}(z)\right]^{2}}{\Delta^{2} \mathcal{A I}_{p_{\mathcal{A} \mathcal{I}}-1}^{\left(q_{\mathcal{A}}\right)}(z)}, \quad p_{\mathcal{A I}} \in \mathbb{N}, q_{\mathcal{A I}} \in \mathbb{N}_{0}
\end{aligned}
$$

where

$$
\Delta \mathcal{A I}_{p_{\mathcal{A I}}-1}^{\left(q_{\mathcal{A} I}\right)}(z)=\mathcal{A I}_{p_{\mathcal{A I}}-1}^{\left(q_{\mathcal{A}}+1\right)}(z)-\mathcal{A I}_{p_{\mathcal{A I}}-1}^{\left(q_{\mathcal{A} \mathcal{I}}\right)}(z), \quad \Delta^{2} \mathcal{A I}_{p_{\mathcal{A I}}-1}^{\left(q_{\mathcal{A}}\right)}(z)=\Delta\left(\Delta \mathcal{A I}_{p_{\mathcal{A I}}-1}^{\left(q_{\mathcal{A} \mathcal{I}}\right)}(z)\right)
$$

Here for given $p_{\mathcal{A I}}$ and $q_{\mathcal{A I}}$, the highest order coefficient of the power series $\hat{x}(z)$ used for the iterative evaluation of $\mathcal{A I}_{p_{\mathcal{A I}}}^{\left(q_{\mathcal{A} I}\right)}(z)$ is given by $\hat{x}_{q_{\mathcal{A I}}+2 p_{\mathcal{A I}}} z^{q_{\mathcal{A I}}+2 p_{\mathcal{A I}}}$.

- Levin's U-transformation (Levin, 1973)

$$
U_{p_{U}}^{\left(q_{U}\right)}(\zeta)=\frac{\sum_{j=0}^{p_{U}}(-1)^{j}\left(\begin{array}{c}
p_{U} \\
j
\end{array}\right) \frac{\left(\zeta+q_{U}+j\right)^{p_{U}-2}}{\left(\zeta+q_{U}+p_{U}\right)^{p_{U}-1}} \frac{s_{q_{U}+j}(z)}{\hat{x}_{q_{U}+j} z^{q_{U}+j}}}{\sum_{j=0}^{p_{U}}(-1)^{j}\left(\begin{array}{c}
p_{U} \\
j
\end{array}\right) \frac{\left(\zeta+q_{U}+j\right)^{p_{U}-2}}{\left(\zeta+q_{U}+p_{U}\right)^{p_{U}-1}} \frac{1}{\hat{x}_{q_{U}+j} z^{q_{U}+j}}}
$$

- Weniger's $\delta$-algorithm (Weniger, 1989) as an analogon to the $d$-transformation of Smith and Ford (1979)

$$
\delta_{p_{\delta}}^{\left(q_{\delta}\right)}(\zeta)=\frac{\sum_{j=0}^{p_{\delta}}(-1)^{j}\left(\begin{array}{c}
p_{\delta} \\
j
\end{array}\right) \frac{\left(\zeta+q_{\delta}+j\right)_{p_{\delta}-1}}{\left(\zeta+q_{\delta}+p_{\delta}\right)_{p_{\delta}-1}} \frac{s_{q_{\delta}+j}(z)}{\hat{x}_{q_{\delta}+j+1} z^{q_{\delta}+j+1}}}{\sum_{j=0}^{p_{\delta}}(-1)^{j}\left(\begin{array}{c}
p_{\delta} \\
j
\end{array}\right) \frac{\left(\zeta+q_{\delta}+j\right)_{p_{\delta}-1}}{\left(\zeta+q_{\delta}+p_{\delta}\right)_{p_{\delta}-1}} \frac{1}{\hat{x}_{q_{\delta}+j+1} z^{q_{\delta}+j+1}}},
$$

where $(a)_{n}$ denotes the Pochhammer symbol i.e. $(a)_{n}=\Gamma(a+n) / \Gamma(a)$.

Both Levin's $U$-transformation and its variants such as Weniger's $\delta$-algorithm are GSTs with explicit remainder estimates. Since in most cases the remainders $R(z, N)$ of an asymptotic expansion or a general series are unknown, a re-construction from a finite and usually small number of partial sums $s_{n}(z), s_{n+1}(z), \ldots, s_{n+m}(z)$ of the known coefficients is used, which typically leads to nonlinear GSTs in terms of the partial sums. It is worth to mention, that often heuristical approaches to determine explicit remainder estimates lead to powerful transformation significantly improving convergence of a given sequence (Levin, 1973). 


\subsubsection{Numerical example: the divergent Euler series}

As a benchmark example to illustrate convergence acceleration and summation of divergent series, recall the Euler series, whose radius of convergence evaluates to zero. In Example 3.2, it is shown that the Euler series $\hat{x}(z)=\sum_{n=0}^{\infty}(-1)^{n} n ! z^{n}$ is $k$-summable for $k>1$ in any direction $d \neq(2 j+1) \pi, j \in \mathbb{Z}$. Furthermore in Section 3.4.2, the $(N, \xi)$-approximate $k$-summation is used to approximate the sum of the Euler series by considering a finite number of series coefficients. In the sequel, summation of the Euler series is considered when applying Aitken's $\Delta^{2}$-algorithm, Levin's $U$-transformation, and Weniger's $\delta$-algorithm. Therefore, the number $N \in \mathbb{N}_{0}$ of series coefficients is varied within $N \in[3,20]$ and compared to the actual solution obtained by evaluation of the Euler integral (3.7) for a given value of $z=1$. The numerical results are summarized in Table 3.3. Clearly, the series diverges as can be seen from the blowup in the partial sums $s_{n}=\sum_{j=0}^{n}(-1)^{j} j$ !. Nevertheless, all algorithms yield highly accurate results for the sum of the series, whereby in particular Weniger's $\delta$-algorithm approximates the sum up to 12 decimal places using 20 series coefficients.

From a computational point of view, the iterated Aitken $\Delta^{2}$-algorithm requires the most computational time given by a factor of approximately 85000 compared to the equally efficient Levin $U$-transformation or Weniger $\delta$-algorithm. As outlined in (Weniger, 1994, p. 112f), the use of double precision is not sufficient such that either quadruple precision or computer algebra systems allowing to achieve a desired numerical precision independent of the machine precision is recommended in order to perform the numerical computations. Hence, MATHEMATICA (Wolfram, 1999) in version 5.0 is used for the numerical implementation.

\subsection{Conclusions}

In this chapter, some properties of formal power series with coefficients in some Banach space are briefly recalled in view of the introduction of Borel and $k$-summation in order to uniquely assign a holomorphic function as the sum to a given possibly divergent formal power series. Furthermore in view of practical application and implementation, a variant, the so-called $(N, \xi)-$ approximate $k$-summation is introduced and exemplarily studied to sum the geometric series outside its circle of convergence and to approximate the limit of the divergent Euler series. For comparison purposes, generalized sequence transformations such as Aitken's $\Delta^{2}$-algorithm, Levin's $U$-transformation, and Weniger's $\delta$-algorithm are briefly discussed and applied to determine the sum of the divergent Euler series. 
Table 3.3: Approximation of the sum (3.7) of the Euler series (3.6) for $z=1$ using Aitken's $\Delta^{2}$ algorithm (3.31), Levin's $U$-transformation (3.32), and Weniger's $\delta$-algorithm (3.33).

\begin{tabular}{l|l|l|l|l}
\hline$N$ & Partial sum $s_{n}$ & $\mathcal{A I}_{[[N / 2]]}^{(N-2[N / 2]])}$ & $U_{N}^{(0)}(\zeta)(1)$ & $\delta_{N}^{(0)}(1)$ \\
\hline \hline 3 & -4 & 0.50000000000000000 & 0.60273972602739726 & 0.59813084112149533 \\
4 & 20 & 0.60714285714285714 & 0.59536275243081525 & 0.59668508287292818 \\
5 & -100 & 0.58181818181818182 & 0.59633396849252570 & 0.59635068980863373 \\
6 & 620 & 0.59778112882378285 & 0.59639917985987091 & 0.59633870078981204 \\
7 & -4420 & 0.59435788011115522 & 0.59633696065850994 & 0.59634540902752171 \\
8 & 35900 & 0.59650983395078321 & 0.59634610226201146 & 0.59634723219620850 \\
9 & -326980 & 0.59610532346036707 & 0.59634834709324914 & 0.59634741922404964 \\
10 & 3301820 & 0.59636273019372916 & 0.59634728313504315 & 0.59634738734962854 \\
11 & -36614980 & 0.59632173652888228 & 0.59634729411250006 & 0.59634736748100896 \\
12 & 442386620 & 0.59634849496958073 & 0.59634738004020485 & 0.59634736257246585 \\
13 & -5784634180 & 0.59634507677394360 & 0.59634736602203161 & 0.59634736205240687 \\
14 & 81393657020 & 0.59634740330055679 & 0.59634736009399440 & 0.59634736219211643 \\
15 & -1226280710980 & 0.59634720669751348 & 0.59634736223541194 & 0.59634736228847645 \\
16 & 19696509177020 & 0.59634735254638192 & 0.59634736256834098 & 0.59634736231882865 \\
17 & -335990918918980 & 0.59634735911419251 & 0.59634736230553450 & 0.59634736232430887 \\
18 & 6066382786809020 & 0.59634735047353435 & 0.59634736229737461 & 0.59634736232415531 \\
19 & $-1.1557872 \times 10^{17}$ & 0.59634736470805851 & 0.59634736232769551 & 0.59634736232356597 \\
20 & $2.3173233 \times 10^{18}$ & 0.59634736243901058 & 0.59634736232588925 & 0.59634736232328641 \\
\hline Sum from $(3.7)$ & 0.59634736232319407 & 0.59634736232319407 & 0.59634736232319407 \\
\hline Accuracy [Decimals] & 9 & 11 & 12 \\
\hline
\end{tabular}




\section{Chapter 4}

\section{Motion planning and feedforward control design using formal power series and summability methods}

The application of formal power series for system inversion and feedforward control design requires special emphasis on the problem of motion planning. This fact is briefly illustrated throughout Sections 2.1 and 2.2 when introducing the notion of FPSP for DCR-systems, i.e. the ability to express states $\boldsymbol{x}(z, t)$ and boundary inputs $\boldsymbol{u}(t)$ of a system of DCR-equations by a parameterizing function $\boldsymbol{y}(t)$ and its time-derivatives up to (possibly) infinite order. As a result, the evaluation of the input parameterization and hence the determination of the respective feedforward control law, requires the specification of desired trajectories $\mathbb{R}^{+} \ni t \mapsto \boldsymbol{y}_{d}(t)$ for $\boldsymbol{y}(t)$ of class $\mathcal{C}^{\infty}$. Furthermore as outlined in Section 2.1, convergence of the formal solution has to be ensured, which leads to the notion of functions of a certain Gevrey order. Additional requirements follow directly from the considered feedforward control task, which in particular for chemical engineering examples such as tubular or fixed-bed reactors includes finite-time transitions between stationary profiles during start-up, operation changes, or shutdown of the reactor.

For the realization of these transitions, a general approach is provided in Section 4.1 using smooth functions having compact support. Following Laroche et al. (2000), this approach can be suitably modified to consider finite-time transitions between arbitrary and not necessarily stationary profiles by the approximation theorem of Stone-Weierstrass. Based on these motion planning results, summability methods and generalized sequence transformations as studied in Section 3.3 are utilized for the feedforward control design for DCR-systems in Section 4.3. Thereby, the different summation techniques are compared by studying divergent formal solutions to the boundary control problem for the linear heat equation introduced in Section 2.1.1 and convergence acceleration of formal solutions of the boundary controlled linear diffusionconvection equation from Section 2.1.2. Following these initial results on scalar systems, Section 4.4 is devoted to the application of summation methods to sum formal solutions originating 
from boundary controlled systems of DCR-equations. In particular it is shown for the tubular reactor model of Section 2.2.2, that divergent formal power series and sophisticated summation approaches provide a powerful tool for motion planning and feedforward tracking control design for nonlinear DCR-systems.

\subsection{Enhanced motion planning in Gevrey classes}

In the sequel, general remarks on the motion planning problem for arbitrary but formal power series parameterizable systems of boundary controlled DCR-equations are provided with the focus on planning trajectories in certain Gevrey classes to ensure smooth transitions within finite time horizons.

\subsubsection{Multiple transitions between stationary profiles}

Since many DCR-systems originate from chemical engineering examples, such as tubular or fixed-bed reactors, typical transition problems concern the finite-time transition between stationary profiles during start-up, operation or shutdown of the reactor.

For the solution of the motion planning problem, the general model description by Eqns. (2.39)(2.42) is re-considered, which is shown to be formal power series parameterizable with state and input parameterization given by Eqns. (2.52)-(2.58). Thereby, the property of FPSP directly provides a solution to the considered control problem of finite-time transitions between an arbitrary number $I_{S}$ of stationary profiles $\boldsymbol{x}_{S}^{i}(z), i=0,1, \ldots, I_{S}$. For the DCR-system (2.39)-(2.42), stationary profiles $\boldsymbol{x}_{S}(z)$ are governed by the following BVP

$$
\begin{aligned}
& S \frac{d^{2} \boldsymbol{x}_{S}(z)}{d z^{2}}+\boldsymbol{\Psi}\left(\boldsymbol{x}_{S}(z), \frac{d \boldsymbol{x}_{S}(z)}{d z}\right)=\mathbf{0}, \quad z \in(0,1) \\
& P_{0} \frac{d \boldsymbol{x}_{S}}{d z}(0)+R_{0} \boldsymbol{x}_{S}(0)=\boldsymbol{\zeta}_{S} \\
& P_{1} \frac{d \boldsymbol{x}_{S}}{d z}(1)+R_{1} \boldsymbol{x}_{S}(1)=Q \boldsymbol{u}_{S}
\end{aligned}
$$

with matrices $S \in \mathbb{R}^{K \times K}, Q \in \mathbb{R}^{M \times M}, P_{0}, R_{0} \in \mathbb{R}^{(2 K-M) \times K}, P_{1}, R_{1} \in \mathbb{R}^{M \times K}$, stationary value of the inhomogenities $\boldsymbol{\zeta}_{S}$, and constant input $\boldsymbol{u}_{S}$. Assuming that the conditions of Theorem 2.4 on FPSP are satisfied, an alternative formulation of the BVP is obtained by replacing the non-homogeneous BCs involving the inputs $\boldsymbol{u}_{S}$ with the stationary version of the imposed BCs (see Section 2.2.1) in terms of stationary values of the parameterizing function $\boldsymbol{y}_{S}$, i.e.

$$
\begin{aligned}
& S \frac{d^{2} \boldsymbol{x}_{S}(z)}{d z^{2}}+\boldsymbol{\Psi}\left(\boldsymbol{x}_{S}(z), \frac{d \boldsymbol{x}_{S}(z)}{d z}\right)=\mathbf{0}, \quad z \in(0,1) \\
& P_{0} \frac{d \boldsymbol{x}_{S}}{d z}(0)+R_{0} \boldsymbol{x}_{S}(0)=\boldsymbol{\zeta}_{S} \\
& F_{d} \frac{d \boldsymbol{x}_{S}}{d z}(0)+F_{v} \boldsymbol{x}_{S}(0)=\boldsymbol{y}_{S} .
\end{aligned}
$$


As a result, stationary solutions $\boldsymbol{x}_{S}(z)$ can be expressed in terms of $\boldsymbol{y}_{S}$ given by the functional dependency $\boldsymbol{x}_{S}(z)=\boldsymbol{x}_{S}\left(z ; \boldsymbol{y}_{S}\right)$. Consequently, re-planning the stationary values $\boldsymbol{y}_{S}$ of the parameterizing function $\boldsymbol{y}(t)$ yields different stationary profiles $\boldsymbol{x}_{S}\left(z ; \boldsymbol{y}_{S}\right)$. Furthermore, the corresponding stationary input values $\boldsymbol{u}_{S}$ can be determined by evaluating the non-homogeneous $\mathrm{BCs}$

$$
\boldsymbol{u}_{S}=Q^{-1}\left[\left.P_{1} \frac{d \boldsymbol{x}_{S}\left(z ; \boldsymbol{y}_{S}\right)}{d z}\right|_{z=1}+R_{1} \boldsymbol{x}_{S}\left(1 ; \boldsymbol{y}_{S}\right)\right]=\boldsymbol{u}_{S}\left(\boldsymbol{y}_{S}\right)
$$

The fact that the stationary solutions satisfy $\boldsymbol{x}_{S}(z)=\boldsymbol{x}_{S}\left(z ; \boldsymbol{y}_{S}\right)$ and $\boldsymbol{u}_{S}=\boldsymbol{u}_{S}\left(\boldsymbol{y}_{S}\right)$, respectively, similarly reflects the notion of FPSP, i.e. the parameterization of states and inputs in terms of the function $\boldsymbol{y}_{S}$.

This approach can be extended to the realization of an arbitrary number of finite-time transitions between stationary profiles for formal power series parameterizable DCR-systems (2.39)(2.42) satisfying (4.1)-(4.3). In view of a representative scenario for motion planning and tracking control, consider the non-isothermal tubular reactor model of Section 2.2.2. As exemplarily illustrated in Figure 4.1, assume it is desired to start the reactor from the initial profile $\boldsymbol{x}_{0}(z)=\boldsymbol{x}_{S}^{0}(z)$, reach an operation profile $\boldsymbol{x}_{S}^{1}(z)$ along a predefined trajectory in finite time, and switch to new stationary profiles $\boldsymbol{x}_{S}^{2}(z)$ and $\boldsymbol{x}_{S}^{3}(z)$ along suitable paths. For the realization of this transition task in view of the functional parameterization $\boldsymbol{x}_{S}\left(z ; \boldsymbol{y}_{S}\right)$, appropriate motion planning requires the design of a desired transient trajectory $\mathbb{R}^{+} \ni t \mapsto \boldsymbol{y}_{d}(t)$ connecting the stationary values $\boldsymbol{y}_{S}^{i}, i=0,1,2,3$ along predefined paths.

In order to meet these objectives, following Fliess et al. (1997) use is made of the $\mathcal{C}^{\infty}$-function $\Phi_{\gamma, T}: \quad \mathbb{R} \rightarrow \mathbb{R}$ defined by Eqns. (B.1), (B.2) of the Appendix B. This function $\Phi_{\gamma, T}(t)$ is non-analytical at $t \in\{0, T\}$, i.e. for $n>0$ the derivatives satisfy $\Phi_{\gamma, T}^{(n)}(t)=0$ for $t \leq 0$ or $t \geq T$ but $\Phi_{\gamma, T}^{(n)}(t) \neq 0$ for $t \in(0, T)$. Due to this property, it is possible to start at $\Phi_{\gamma, T}(0)=0$ and reach exactly the desired value $\Phi_{\gamma, T}(t \geq T)=1$ within the finite-time interval $t \in[0, T]$. Further properties of the smooth compact support function $\Phi_{\gamma, T}(t)$ are derived in Lynch and Rudolph (2002), where in particular its Gevrey order is determined as $\alpha=1+\frac{1}{\gamma}$. Note that there is no solution to the control problem using an analytic function, since an analytic function being constant on an interval of its domain is necessarily constant due to Liouville's theorem (Rudin, 1999, p. 253). Hence, no transition is possible in this case.

Combining these results for $\Phi_{\gamma, T}(t)$, the desired trajectory $\boldsymbol{y}_{d}(t)=\left[y_{1, d}(t), \ldots, y_{M, d}(t)\right]^{T}$ for the parameterizing function $\boldsymbol{y}(t)$ is assigned as

$$
y_{j, d}(t)=y_{j, S}^{0}+\left(y_{j, S}^{1}-y_{j, S}^{0}\right) \Phi_{\gamma_{j}^{1}, T_{j}^{1}-T_{j}^{0}}\left(t-T_{j}^{0}\right)+\left(y_{j, S}^{2}-y_{j, S}^{1}\right) \Phi_{\gamma_{j}^{2}, T_{j}^{3}-T_{j}^{2}}\left(t-T_{j}^{2}\right)
$$

for $j \in\{1,2, \ldots, M\}=: J_{M}$. This approach can be easily generalized to an arbitrary number of transitions

$$
y_{j, S}(t)=y_{j, S}^{0}+\sum_{i=0}^{I_{S}^{j}}\left(y_{j, S}^{i+1}-y_{j, S}^{i}\right) \Phi_{\gamma_{j}^{i+1}, T_{j}^{2 i+1}-T_{j}^{2 i}}\left(t-T_{j}^{2 i}\right), \quad j \in J_{M}
$$


between stationary profiles. Note that the trajectories for any $y_{j, d}(t) j \in J_{M}$ can be chosen independently, such that depending on the transition times $T_{j}^{i}, i=0,1, \ldots, I_{S}^{j} ; j \in J_{M}$, a maximum number of $1+M \sum_{j=1}^{M} I_{S}^{j}$ operating profiles can be realized by (4.6). Desired trajectories for $\boldsymbol{y}_{d}(t)$ with $M=2$ are exemplarily shown in Figure 4.1. In particular, due to the possibility of an independent assignment of trajectories for each component of $\boldsymbol{y}_{d}(t)$, several intermediate states can be reached.

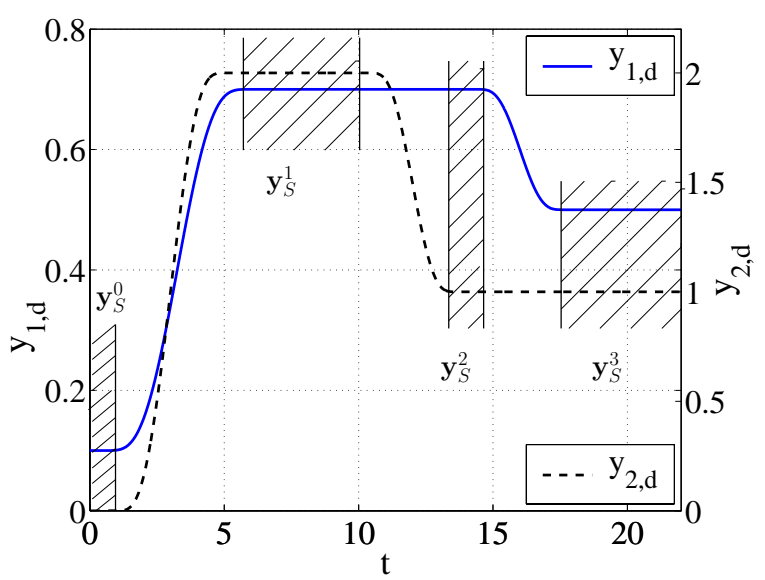

Figure 4.1: Desired trajectories $y_{j, d}(t)=\left[y_{1, d}(t), y_{2, d}(t)\right]^{T}$ for the realization of transitions between the 4 dashed operating points. The design parameters for the evaluation of (4.5) are summarized in Table D.1 of the Appendix D.2.

\subsubsection{Multiple transitions between arbitrary profiles}

The previous approach for motion planning can be suitably modified to realize finite-time transitions between arbitrary and in particular not necessarily stationary profiles as proposed by Laroche et al. (2000). In order to illustrate their approach, the scalar case, i.e. $K=M=1$ is considered, whereby the calculations directly carry over to the case $K, M>1$. Hence, given an initial profile

$$
x(z, 0)=x_{0}(z), \quad z \in[0,1],
$$

a terminal profile at time $t=T>0$

$$
x(z, T)=x_{T}(z), \quad z \in[0,1],
$$

and assume that $x_{0}(z), x_{T}(z) \in \boldsymbol{L}_{2}(0,1)$, then by the Stone-Weierstrass Theorem (Rudin, 1976), there exist polynomials

$$
P_{0}(z)=\sum_{n=0}^{N_{p}} \check{p}_{n} z^{n}, \quad P_{T}(z)=\sum_{n=0}^{N_{p}} \check{q}_{n} z^{n}
$$


such that $\left\|x_{0}-P_{0}\right\|<\epsilon$ and $\left\|x_{T}-P_{T}\right\|<\epsilon$, for all $\epsilon>0$ where $\|\cdot\|$ denotes the norm in $\boldsymbol{L}_{2}(0,1)$. As a direct consequence (Laroche et al., 2000), the choice of

$$
y_{d}(t):=\left(1-\Phi_{\gamma, T}(t)\right) \sum_{n=0}^{N_{p}} \check{p}_{n} \frac{t^{n}}{n !}+\Phi_{\gamma, T}(t) \sum_{n=0}^{N_{p}} \check{q}_{n} \frac{(t-T)^{n}}{n !}
$$

provides a trajectory $y_{d}(t)$ which allows an approximate realization of the transition from the initial profile $x_{0}(z)$ to the final profile $x_{T}(z)$ in time $0<t \leq T$. Note that either profile can be only reached approximately, since the number $M$ of boundary inputs is finite, whereby each profile contains an infinite number of degrees-of-freedom since $z \in \mathbb{R}^{+}$. As a result, at most $M$ (here 1) independent trajectories can be assigned.

Since

$$
\begin{array}{llll}
y_{d}^{(j)}(0)=\check{p}_{j}, & y_{d}^{(j)}(T)=\check{q}_{j}, & & j=0,1, \ldots, N_{p} \\
y_{d}^{(j)}(0)=0, & y_{d}^{(j)}(T)=0, & j>N_{p},
\end{array}
$$

the parameterizations (2.52)-(2.55) of the differential recursion (2.47) for $K=M=1$ in terms of $y(t)$ and its time-derivatives at times $t=0, T$ reduce to

$$
\begin{aligned}
\hat{x}_{2 n+m}^{d}(0) & =\Upsilon_{2 n+m}^{d, 0}\left(\left[\check{p}_{0}, \ldots, \check{p}_{j}\right]^{T}\right), & j \leq N_{p} \\
\hat{x}_{2 n+m}^{d}(T) & =\Upsilon_{2 n+m}^{d, T}\left(\left[\check{q}_{0}, \ldots, \check{q}_{j}\right]^{T}\right), & \\
\hat{x}_{2 n+m}^{d}(0) & =\Upsilon_{2 n+m}^{d, 0}\left(\left[\check{p}_{0}, \ldots, \check{p}_{N_{p}}, 0, \ldots, 0\right]^{T}\right), & j>N_{p} \\
\hat{x}_{2 n+m}^{d}(T) & =\Upsilon_{2 n+m}^{d, T}\left(\left[\check{q}_{0}, \ldots, \check{q}_{N_{p}}, 0, \ldots, 0\right]^{T}\right) &
\end{aligned}
$$

with $m=0,1$. Hence, the corresponding spatial profiles follow as

$$
\hat{x}^{d}(z, 0)=\sum_{n=0}^{\infty} \Upsilon_{n}^{d, 0}(\check{\boldsymbol{p}}) z^{n}, \quad \hat{x}^{d}(z, T)=\sum_{n=0}^{\infty} \Upsilon_{n}^{d, T}(\check{\boldsymbol{q}}) z^{n}
$$

with $\check{\boldsymbol{p}}=\left[\check{p}_{0}, \ldots, \check{p}_{N_{p}}\right]^{T}$ and $\check{\boldsymbol{q}}=\left[\check{q}_{0}, \ldots, \check{q}_{N_{p}}\right]^{T}$. Naturally the question arises whether $\| x^{d}(z, 0)-$ $x_{0} \|<\epsilon$ and $\left\|\hat{x}^{d}(z, T)-x_{T}\right\|<\epsilon$ with $\epsilon \ll 1$. In case of convergency of the formal solution, the answer is trivial by definition. Nevertheless, it is observed in Section 2.1.2 for the boundary controlled linear diffusion-convection equation by studying variations in the convection rate, that although convergence is ensured, the respective speed of convergence might be small. Since in addition only a finite number of series coefficients can be summed for the series evaluation, it can be expected that the approximation error $\epsilon$ in the inequalities above might possibly be large depending on the number of addends. For the study of weakly nonlinear DCR-equations, the situation becomes even worse due to convergence restrictions relating motion planning and system parameters as illustrated in Section 2.1.3.

Thus in view of a wide range application and realization of the desired motion, the restriction to (uniformly) convergent power series — or equivalently partial summation as a summation method - has to be replaced by studying regular summation methods prolonging the space of (uniformly) convergent power series. In the sequel, it will be shown that $k$-summation and 
its proposed variant, the $(N, \xi)$-approximate $k$-summation studied in Sections 3.15 and 3.4 provide a highly efficient and powerful tool for enhanced motion planning and feedforward control design for a broad class of nonlinear DCR-systems. In view of their application to PDEs, the considerations of Chapter 3 have to be adapted, as is summarized below.

\subsection{Power series solutions and summability methods for PDEs}

The classical results on power series solutions for PDEs are typically related to the Kowalewskian case on convergent analytic solutions to the initial-value problem (IVP) (Haack and Wendland, 1969). Nevertheless, new interest has arisen in the non-Kowalewskian case of solutions with radius of convergence equal to zero. Various authors study the Gevrey order for such power series (see e.g. (Gérard and Tahara, 1998) and the references therein). In addition, it can be shown that the resulting formal solutions are asymptotic representations of proper solutions of the underlying equations (Ouchi, 1994). Recent work concerns the questions of $k$-summability and multi-summability of these series. Thereby, a complete summability analysis is available for the Cauchy problem to the linear heat equation (Lutz et al., 1996; Balser, 1999). In subsequent articles, several other PDEs are studied with either constant (Balser and Miyake, 1999) or holomorphic coefficients (Hibino, 2001, 2003). For an extensive literature survey, see e.g. (Balser, 2003), where summability of the Cauchy problem to the inhomogeneous heat equation is studied, or (Balser and Malek, 2003) on the multi-dimensional heat equation. Recent results on formal solutions to ODEs and PDEs are also summarized in (Balser and Kostov, 2003).

Thereby it is interesting to note that the mathematical and physical literature on formal power series is focused on the study of the Cauchy problem, while typically neglecting the case of initial-boundary-value problems, which naturally arise in the study of boundary control problems for PDEs. Based on the initial work of e.g. Fliess et al. (1997, 1998a,b); Laroche et al. (1998); Laroche (2000); Lynch and Rudolph (2000a, 2002) on uniformly convergent series solutions to IBVPs arising from control applications, new results where obtained within the general framework of parameterization:

- scalar PDEs with temporally and spatially varying as well as state dependent coefficients (Rothfuß et al., 2000; Lynch and Rudolph, 2002),

- nonlinear PDEs with quadratic (Lynch and Rudolph, 2000a, 2002) and cubic nonlinearity (Meurer and Zeitz, 2004b) — see also Section 2.1.3 of this thesis,

- coupled sets of PDEs modeling a tubular reactor with quadratic nonlinearity (Lynch and Rudolph, 2000c), (Becker, 2003), (Meurer et al., 2003),

- nonlinear Stefan problems with moving BCs (Dunbar et al., 2003). 
For a comprehensive study, the reader is also referred to the work of Rudolph (2003a,b); Rudolph et al. (2003). As illustrated in Chapter 2, these results share the fact that convergence greatly depends on appropriate motion planning, i.e. restricting the desired trajectories to smooth function of Gevrey order $\alpha \in(1,2]$.

Besides the convergent case, Laroche et al. (2000) illustrate that also pointwise or possibly divergent series solutions might be useful for feedforward control design by considering the boundary controlled linear heat equation. Hence in view of the literature on summability results for divergent solutions to certain linear and nonlinear IVPs, an extension of these results to IBVPs seems promising. Initial impulses towards this study are provided by Wagner (2003) and Balser $(2003 / 2004,2004)$, with the latter reference providing an analysis of $k$-summability for divergent power series solutions to the boundary controlled heat equation in the complex domain. Nevertheless also for the simplest cases, the summability analysis is far from being complete.

On the other hand and from an application point of view, the numerical results of Section 3.4 for the proposed variant of $k$-summation, the so-called $(N, \xi)$-approximate $k$-summation, illustrate that it is still possible to re-construct a meaningful limit to a given divergent series by appropriately combining a finite number of addends. Similar results are obtained using generalized sequence transformation, which are extensively studied e.g. in quantum physics and quantum chemistry (Zinn-Justin, 1989; Weniger, 1994). In the following, these techniques are exploited for motion planning and feedforward control design for DCR-equations with boundary input.

\subsection{Scalar DCR-equations with boundary input}

In the following, the introduced $(N, \xi)$-approximate $k$-summation as well as the considered prototype examples of generalized sequence transformations, namely Aitken's algorithm and Weniger's $\delta$-transformation, are applied for enhanced feedforward control design for the scalar boundary controlled DCR-equations of Section 2.1. Thereby, the section is focused on the numerical application and implementation.

\subsubsection{Enhanced motion planning for the linear heat equation}

As an illustrative benchmark example, consider first the linear heat equation which is studied in Section 2.1.1 in the context of operational calculus and formal power series parameterizability. In particular, it is shown in Theorem 2.1, that the formal solution converges if the parameterizing function $y(t)=x(0, t)$ is Gevrey of order $\alpha \leq 2$. On the other hand, the numerical results in Fig. 2.1 indicate, that divergent solutions by assigning trajectories $t \mapsto y_{d}(t)$ for $y(t)$ of Gevrey order $\alpha>2$ yield smoother finite-time transitions with lower input amplitude. Nevertheless, meaningful results using classical partial summation up to the available number 

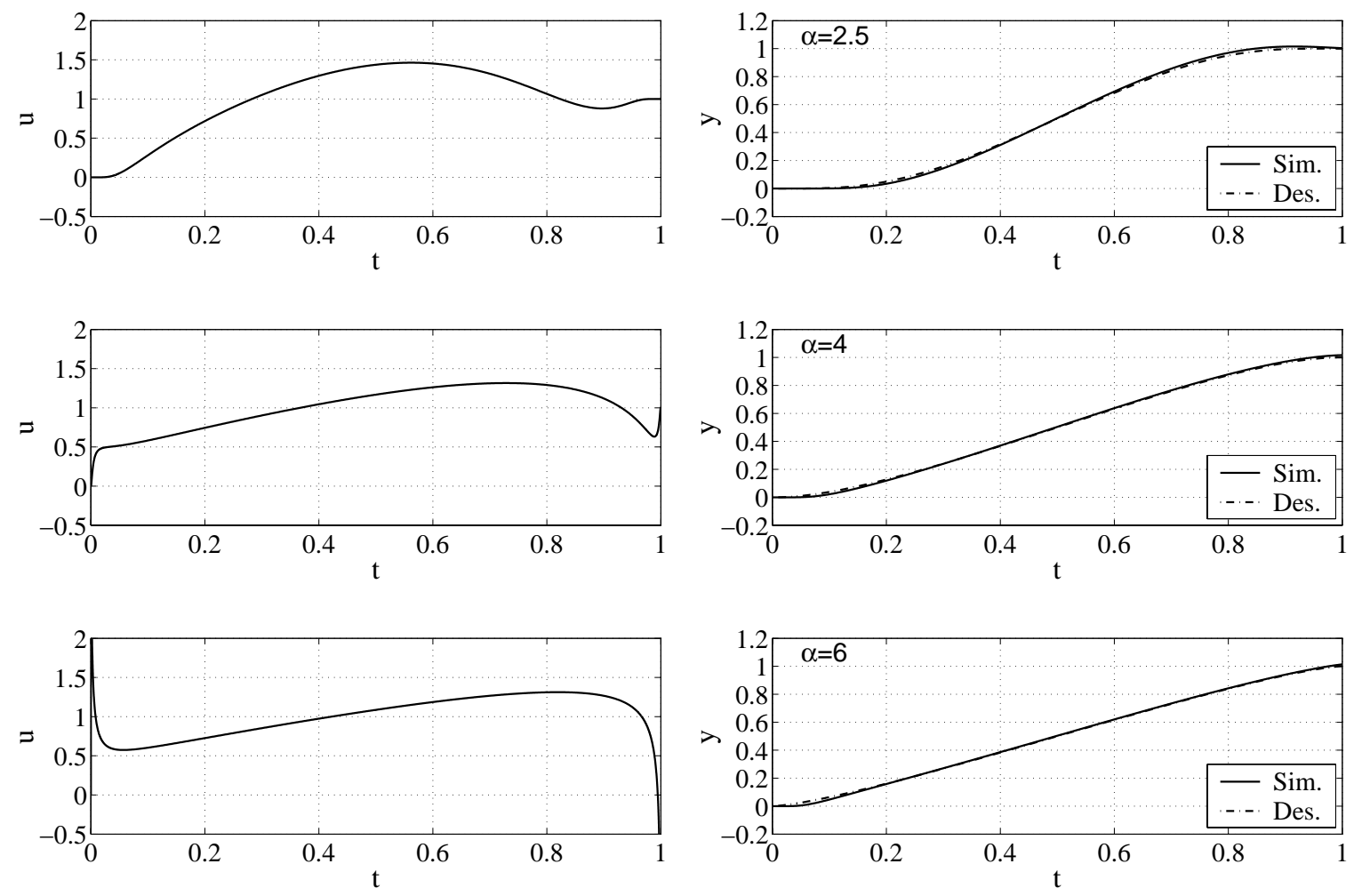

Figure 4.2: Open-loop control of the linear heat equation (2.1)-(2.4) using Aitken's algorithm (3.31) for $\lambda=1, \nu=0, \beta=0, p_{1}=0, r_{1}=1, \Psi(x)=0$ with desired trajectory $y_{d}(t)=\Phi_{\gamma, T}(t)($ B.1) of Gevrey order $1+1 / \gamma=\alpha \in\{2.5,4,6\}$ for $T=1$. Left: feedforward control $u_{N_{\mathcal{S}}}(t)$ from (2.17); right: output $y(t)=x(0, t)$ compared to desired trajectory $y_{d}(t)$.

of series coefficients are only obtained for Gevrey orders close to $\alpha=2$, whereby already for $\alpha=2.5$ the divergent behavior becomes apparent - see Figure 2.1.

Note that this is already observed in Laroche et al. (2000), where least-term summation is used to determine the limit of the divergent series. As illustrated in Section 3.2.2, the least-term summation (3.8) approach can be deduced from asymptotic expansions and the observation that many divergent series behave like convergent ones before growing beyond any bound. Although the results of Laroche et al. (2000) yield the applicability of least-term summation for the linear heat equation, the summation results cannot be recovered for nonlinear problems (Wagner, 2003), (Wagner et al., 2004), such that the least-term summation approach is not considered within this contribution.

In order to overcome these limitations, the advanced summation processes studied in Chapter 3 are applied to enhance motion planning for the boundary controlled linear heat equation and to determine the respective feedforward control. Therefore, Aitken's algorithm $\mathcal{A I}_{p_{\mathcal{A I}}}^{\left(q_{\mathcal{I}}\right)}(3.31)$ as the prototype of iterative approaches, Weniger's $\delta$-algorithm $\delta_{p_{\delta}}^{\left(q_{\delta}\right)}(3.33)$ as a successor of the Levin transformations, and the proposed $(N, \xi)$-approximate $k$-sum $\mathcal{S}_{k}^{(N, \xi)}(3.26)$ are applied 

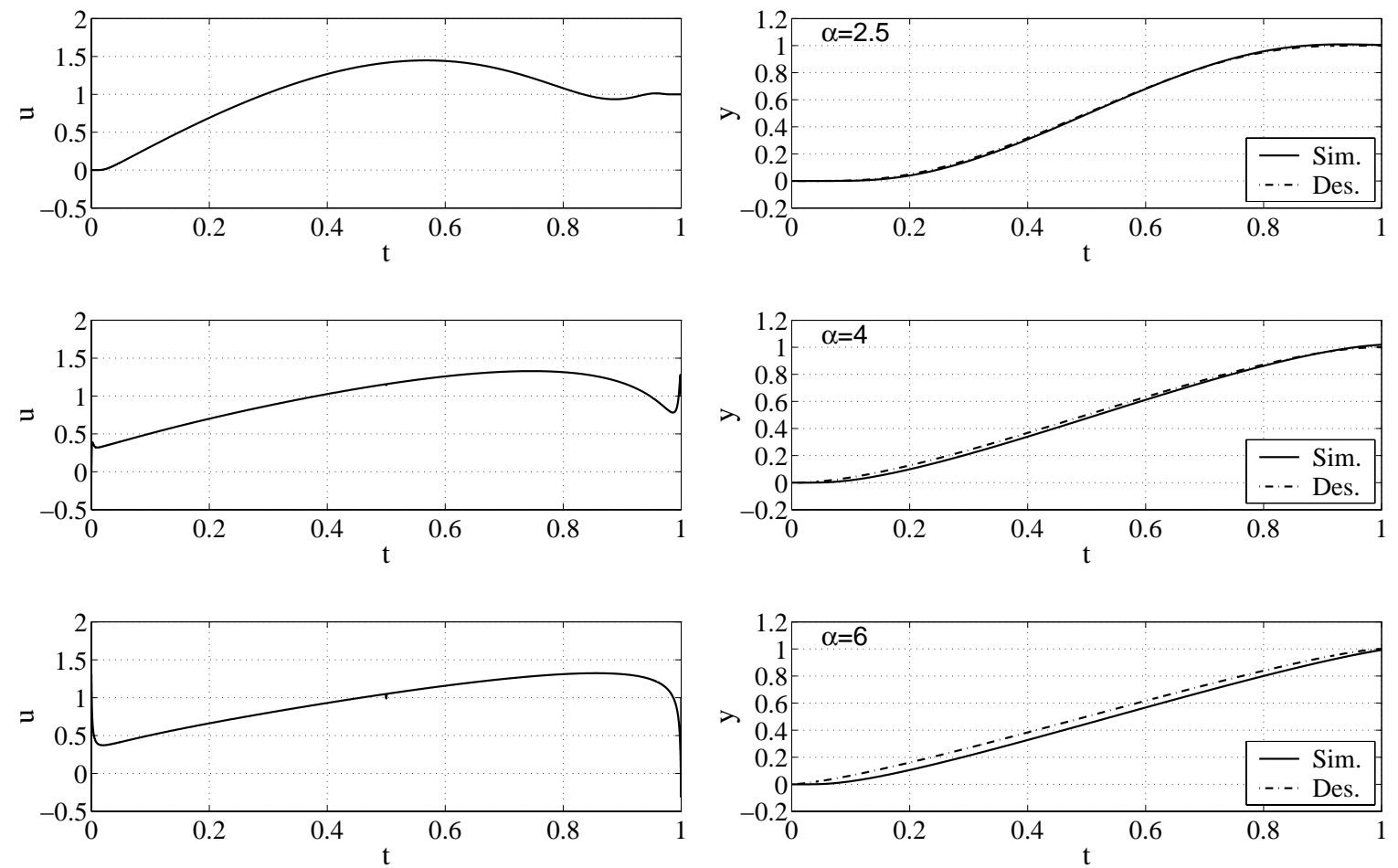

Figure 4.3: Open-loop control of the linear heat equation (2.1)-(2.4) using Weniger's $\delta$-algorithm (3.33) for $\lambda=1, \nu=0, \beta=0, p_{1}=0, r_{1}=1, \Psi(x)=0$ with desired trajectory $y_{d}(t)=\Phi_{\gamma, T}(t)(B .1)$ of Gevrey order $1+1 / \gamma=\alpha \in\{2.5,4,6\}$ for $T=1$. Left: feedforward control $u_{N_{\mathcal{S}}}(t)$ from (2.17); right: output $y(t)=x(0, t)$ compared to desired trajectory $y_{d}(t)$.

to the formal power series parameterization of the linear heat equation (2.21), (2.22) i.e.

$$
\begin{aligned}
\hat{x}_{2 n}(t) & =\frac{1}{\lambda^{n}(2 n) !} \sum_{i=0}^{n}\left(\begin{array}{c}
n \\
i
\end{array}\right)(-\beta)^{n-i} y^{(i)}(t), \\
\hat{x}_{2 n+1}(t) & =0, .
\end{aligned}
$$

where $y(t)=x(0, t)$. Using $\hat{x}(z, t)=\sum_{n=0}^{\infty} \hat{x}_{n}(t) z^{n}$, the boundary control $u(t)=p_{1} \partial x / \partial z(1, t)+$ $r_{1} x(1, t)$ can be formally determined as

$$
\hat{u}(t)=r_{1} \hat{x}_{0}(t)+\sum_{n=1}^{\infty}\left(r_{1}+n p_{1}\right) \hat{x}_{n}(t)=: \sum_{n=0}^{\infty} \hat{u}_{n}(t) .
$$

By Theorem 2.1, this series converges for $y(t)$ being Gevrey of order $\alpha \leq 2$. However in view of numerical evaluation and implementation, the infinite series has to be truncated at some integer. It is thereby desired to recover the limit of the series $\hat{x}(z, t)$ and $\hat{u}(t)$, respectively, for the convergent as well as the divergent case by increasing the Gevrey order of the desired trajectory. Application of the considered summation approaches yields

$$
u_{N_{\mathcal{S}}}(t)=\mathcal{S}\left(\sum_{n=0}^{N_{\mathcal{S}}} \hat{u}_{n}(t)\right)
$$



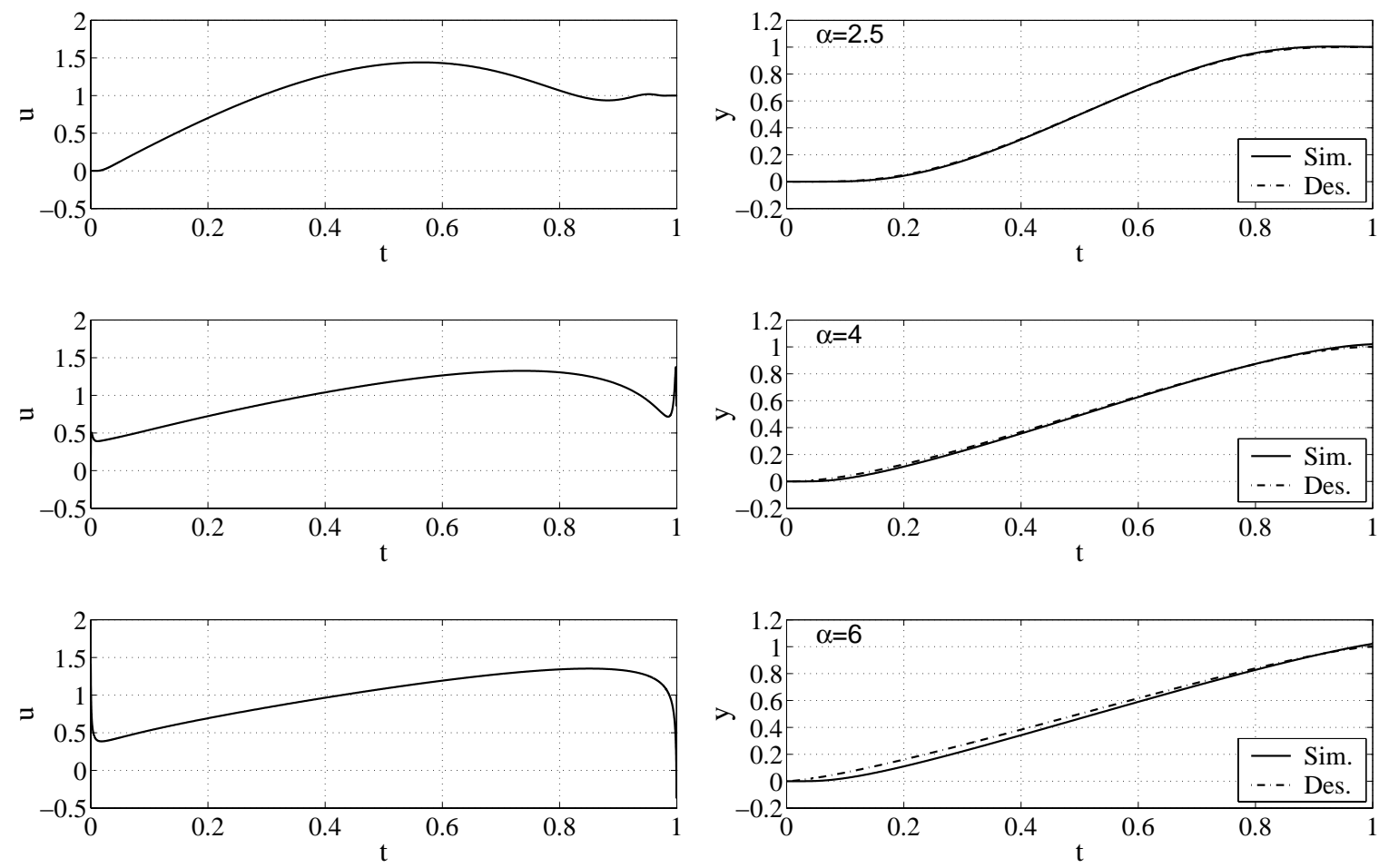

Figure 4.4: Open-loop control of the linear heat equation (2.1)-(2.4) using $\left(N_{\mathcal{S}}, \xi\right)$-approximate $k^{-}$ $\operatorname{sum}(3.26)$ for $\lambda=1, \nu=0, \beta=0, p_{1}=0, r_{1}=1, \Psi(x)=0$ with desired trajectory $y_{d}(t)=\Phi_{\gamma, T}(t)$ (B.1) of Gevrey order $1+1 / \gamma=\alpha \in\{2.5,4,6\}$ for $T=1$. Left: feedforward control $u_{N_{\mathcal{S}}}(t)$ from (2.17); right: output $y(t)=x(0, t)$ compared to desired trajectory $y_{d}(t)$.

where $\mathcal{S} \in\left\{\mathcal{A I}_{p_{\mathcal{A I}}}^{\left(q_{\mathcal{A} \mathcal{I}}\right)}, \delta_{p_{\delta}}^{\left(q_{\delta}\right)}, \mathcal{S}_{k}^{(N, \xi)}\right\}$ with $N_{\mathcal{S}}=N_{\mathcal{S}}(\mathcal{S})$. Numerical results when varying the Gevrey order $\alpha \in\{2.5,4,6\}$ are depicted in Figures $4.2-4.4$ for $\mathcal{A}_{p_{\mathcal{A I}}}^{\left(q_{\mathcal{A}}\right)}, \delta_{p_{\delta}}^{\left(q_{\delta}\right)}$, and $\mathcal{S}_{k}^{(N, \xi)}$, respectively. Thereby, the desired trajectory $t \mapsto y_{d}(t)$ is chosen as $\Phi_{1 /(\alpha-1), 1}(t)$ defined in (B.1) of Appendix B. The corresponding feedforward controls $u_{N_{\mathcal{S}}}(t)$ obtained from the summation approaches (respective left column) are applied to a MOL discretized model of the boundary controlled heat conduction system in order to compare the output $y(t)$ with its desired values $y_{d}(t)$ (respective right column). The integer $N_{\mathcal{S}}$ is adapted to each summation process, with $N_{\mathcal{S}}\left(\mathcal{A I}_{p_{\mathcal{A I}}}^{\left(q_{\mathcal{I}}\right)}\right)=q_{\mathcal{A I}}+2 p_{\mathcal{A I}}, N_{\mathcal{S}}\left(\delta_{p_{\delta}}^{\left(q_{\delta}\right)}\right)=p_{\delta}+q_{\delta}+1, N_{\mathcal{S}}\left(\mathcal{S}_{k}^{(N, \xi)}\right)=39$, and remaining summation parameters summarized in Table 4.1.

Obviously, the three approaches yield sufficiently accurate results for these highly divergent scenarios, proving their applicability to sum divergent series to a meaningful limit. However for increasing Gevrey order $\alpha$, the inputs reach their maximum close to the boundaries at $t \in\{0, T\}$, which reflect the behavior of the desired trajectory whose derivatives exhibit their maximum near $t=0$ and $t=T$ for $\alpha>2-$ see Appendix B.

Nevertheless it should be pointed out, that the computation time for the iterated $\mathcal{A I}_{p_{\mathcal{A I}}}^{\left(q_{\mathcal{A I}}{ }_{-}\right.}$ algorithm exceeds the time for both $\delta_{p_{\delta}}^{\left(q_{\delta}\right)}$ and $\mathcal{S}_{k}^{(N, \xi)}$ by several orders of magnitude. Furthermore, an application of $\mathcal{A I}_{p_{\mathcal{A I}}}^{\left(q_{\mathcal{A} \mathcal{I}}\right)}$ and $\delta_{p_{\delta}}^{\left(q_{\delta}\right)}$ requires to perturb some of the series coefficients $\hat{u}_{n}(t)$ 
Table 4.1: Parameters $p_{\mathcal{A I}}, q_{\mathcal{A I}}$ of Aitken's algorithm (3.31), $p_{\delta}, q_{\delta}, \zeta$ of Weniger's $\delta$-algorithm (3.33), and $\xi$ and $k$ of $\left(N_{\mathcal{S}}, \xi\right)$-approximate $k$-sum (3.26), when applied for open-loop control design for the linear heat equation (2.1)-(2.4) with parameters $\lambda=1, \nu=0, \beta=0, p_{1}=0, r_{1}=1, \Psi(x)=0$ and desired trajectory of Gevrey order $\alpha \in\{2.5,4,6\}$.

\begin{tabular}{l|l|l|l|l|l|l|l}
\hline$\alpha$ & $p_{\mathcal{A I}}$ & $q_{\mathcal{A I}}$ & $p_{\delta}$ & $q_{\delta}$ & $\zeta$ & $\xi$ & $k$ \\
\hline \hline 2.5 & 3 & 1 & 10 & 0 & 150 & 1000 & $2 / 5$ \\
\hline 4 & 3 & 1 & 7 & 0 & $10^{4}$ & $10^{5}$ & $1 / 4$ \\
\hline 6 & 3 & 1 & 5 & 0 & 2500 & $1.2 \times 10^{7}$ & $1 / 6$ \\
\hline
\end{tabular}

by some $\epsilon \ll 1$ due their zero value over some intervals of time, which in some cases induces further problems for numerical evaluation. This can be easily deduced from the governing equations (3.31) and (3.33), respectively, where the denominator directly depends on the series coefficients. In addition, it can be observed that numerical overflow using double precision is a typical problem in particular for Weniger's $\delta$-algorithm, such that the use of quadruple precision or computer-algebra-systems with arbitrary working precision is recommended. Note that these drawbacks of the considered generalized sequence transformations are not observed using the $\left(N_{\mathcal{S}}, \xi\right)$-approximate $k$-sum, which under the assumption of suitably chosen parameters $\xi$ and $k$ for given $N_{\mathcal{S}}$ yields a highly accurate result for the feedforward term to realize the desired transition.

\subsubsection{Convergence acceleration for the linear diffusion-convection equation}

In Section 2.1.2, the applicability of formal power series to study the boundary controlled linear diffusion-convection equation is outlined within the framework of formal power series parameterizability. Thereby, system state $x(z, t)$ and boundary input $u(t)$ are parameterized in terms of the parameterizing function $y(t)=x(0, t)$, such that the series coefficients $\hat{x}_{n}(t), n \in$ $\mathbb{N}_{0}$ of $\hat{x}(z, t)=\sum_{n=0}^{\infty} \hat{x}_{n}(t) z^{n}$ can be determined from the differential recursion $(2.24)-(2.27)$, i.e.

$$
\begin{aligned}
\hat{x}_{n+2}(t) & =\frac{\dot{\hat{x}}_{n}(t)-\nu(n+1) \hat{x}_{n+1}(t)-\beta \hat{x}_{n}(t)}{\lambda(n+2)(n+1)}, \quad n \in \mathbb{N}_{0} \\
\hat{x}_{1}(t) & =0, \quad \hat{x}_{0}(t)=y(t) .
\end{aligned}
$$

For this example, a closed form solution of the differential recursion in terms of $y(t)$ and its time-derivatives up to infinite order is available by Eqn. (2.28). On the other hand, evaluation of $\mathrm{BC}(2.4)$ involving the input $u(t)$, yields its formal power series expansion

$$
\hat{u}(t)=r_{1} \hat{x}_{0}(t)+\sum_{n=1}^{\infty}\left(r_{1}+n p_{1}\right) \hat{x}_{n}(t)=: \sum_{n=0}^{\infty} \hat{u}_{n}(t) .
$$


Assigning trajectories $[0, T] \ni t \mapsto y_{d}(t) \in G_{M, R, \alpha}([0, T])$ with $\alpha \leq 2$, i.e. trajectories of Gevrey order $\alpha \leq 2$, by Theorem 2.2 uniform convergence of the formal solution can be verified with at least a unit radius of convergence. Nevertheless, the proof of convergency does not account for the speed of convergence of the formal solution as illustrated in Figure 2.2. In particular, for high values of the convection parameter $\nu$, a large number of series coefficients is required to obtain a useful solution.

In order to overcome this limitation, the summation processes studied in Sections 3.4 and 3.5 are applied to determine a meaningful limit to the series

$$
u_{N_{\mathcal{S}}}(t)=\mathcal{S}\left(\sum_{n=0}^{N_{\mathcal{S}}} \hat{u}_{n}(t)\right)
$$

using only a finite number of series coefficients accessible for numerical evaluation. Similar to the previous section, Aitken's algorithm $\mathcal{A I}_{p_{\mathcal{A I}}}^{\left(q_{\mathcal{A}}\right)}$ (3.31), Weniger's $\delta$-algorithm $\delta_{p_{\delta}}^{\left(q_{\delta}\right)}(3.33)$, and the $\left(N_{\mathcal{S}}, \xi\right)$-approximate $k$-sum $\mathcal{S}_{k}^{\left(N_{\mathcal{S}}, \xi\right)}(3.26)$ are applied to the sequence $\left\{\hat{u}_{n}(t)\right\}_{n=0}^{N_{\mathcal{S}}}$ for $t \in[0, T]$, such that $\mathcal{S} \in\left\{\mathcal{A I}_{p_{\mathcal{A I}}}^{\left(q_{\mathcal{A}}\right)}, \delta_{p_{\delta}}^{\left(q_{\delta}\right)}, \mathcal{S}_{k}^{\left(N_{\mathcal{S}}, \xi\right)}\right\}$. Numerical results for the boundary controlled linear diffusion-convection system (2.1)-(2.4) when increasing the convection rate $\nu \in\{10,20,25\}$ with $\lambda=1, \beta=0, p_{1}=0, r_{1}=1, \Psi(x)=0$ are depicted in Figures 4.5-4.8 for $\mathcal{A I}_{p_{\mathcal{A I}}}^{\left(q_{\mathcal{I}}\right)}$, $\delta_{p_{\delta}}^{\left(q_{\delta}\right)}$, and $\mathcal{S}_{k}^{(N, \xi)}$, respectively. Thereby, the feedforward control $u_{N_{\mathcal{S}}}(t)$ determined from the summation processes (respective left column) is applied to a MOL discretized model of the boundary controlled diffusion-convection system in order to compare the output $y(t)$ with its desired values $y_{d}(t)$ (respective right column). The integer $N_{\mathcal{S}}$ is adapted to each summation process, with $N_{\mathcal{S}}\left(\mathcal{A I}_{p_{\mathcal{A I}}}^{\left(q_{\mathcal{A} \mathcal{I}}\right)}\right)=q_{\mathcal{A I}}+2 p_{\mathcal{A I}}, N_{\mathcal{S}}\left(\delta_{p_{\delta}}^{\left(q_{\delta}\right)}\right)=p_{\delta}+q_{\delta}+1$ and $N_{\mathcal{S}}\left(\mathcal{S}_{k}^{(N, \xi)}\right)=39$ with the remaining summation parameters summarized in Table 4.2.

The results clearly illustrate the applicability of Aitken's algorithm also in the case of high convection rates. Note that the parameters entering the algorithm have to be chosen with special care depending on the value of $\nu$, since small variations in either $p_{\mathcal{A I}}$ or $q_{\mathcal{A I}}$ result in large differences in the numerical results. For clarification, Figure 4.6 shows the feedforward control $u_{N_{\mathcal{S}}}(t)$ for the case of $\nu=20$ determined by $\mathcal{A I}_{p_{\mathcal{A I}}}^{\left(q_{\mathcal{A} I}\right)}$ with a varied value of $p_{\mathcal{A I}} \in\{3,5\}$

Table 4.2: Parameters $p_{\mathcal{A I}}, q_{\mathcal{A} \mathcal{I}}$ of Aitken's algorithm (3.31), $p_{\delta}, q_{\delta}, \zeta$ of Weniger's $\delta$-algorithm (3.33), and $\xi$ and $k$ of $\left(N_{\mathcal{S}}, \xi\right)$-approximate $k$-sum (3.26), when applied for open-loop control design for the linear diffusion-convection equation (2.1)-(2.4) with $\nu$ varied while $\lambda=1, \beta=0, p_{1}=0, r_{1}=1, \Psi(x)=0$.

\begin{tabular}{|c|c|c|c|c|c|c|c|}
\hline$\nu$ & $p_{\mathcal{A I}}$ & $q_{\mathcal{A I}}$ & $p_{\delta}$ & $q_{\delta}$ & $\zeta$ & $\xi$ & $k$ \\
\hline 10 & 30 & 2 & 38 & 0 & 1 & 20 & 1 \\
\hline 20 & 4 & 4 & 38 & 0 & 1 & 20 & 1 \\
\hline 25 & 4 & 4 & 38 & 0 & 1 & 10 & 1 \\
\hline 50 & \multicolumn{5}{|c|}{-} & 5 & 1 \\
\hline 100 & \multicolumn{5}{|c|}{ - } & 2 & 1 \\
\hline
\end{tabular}



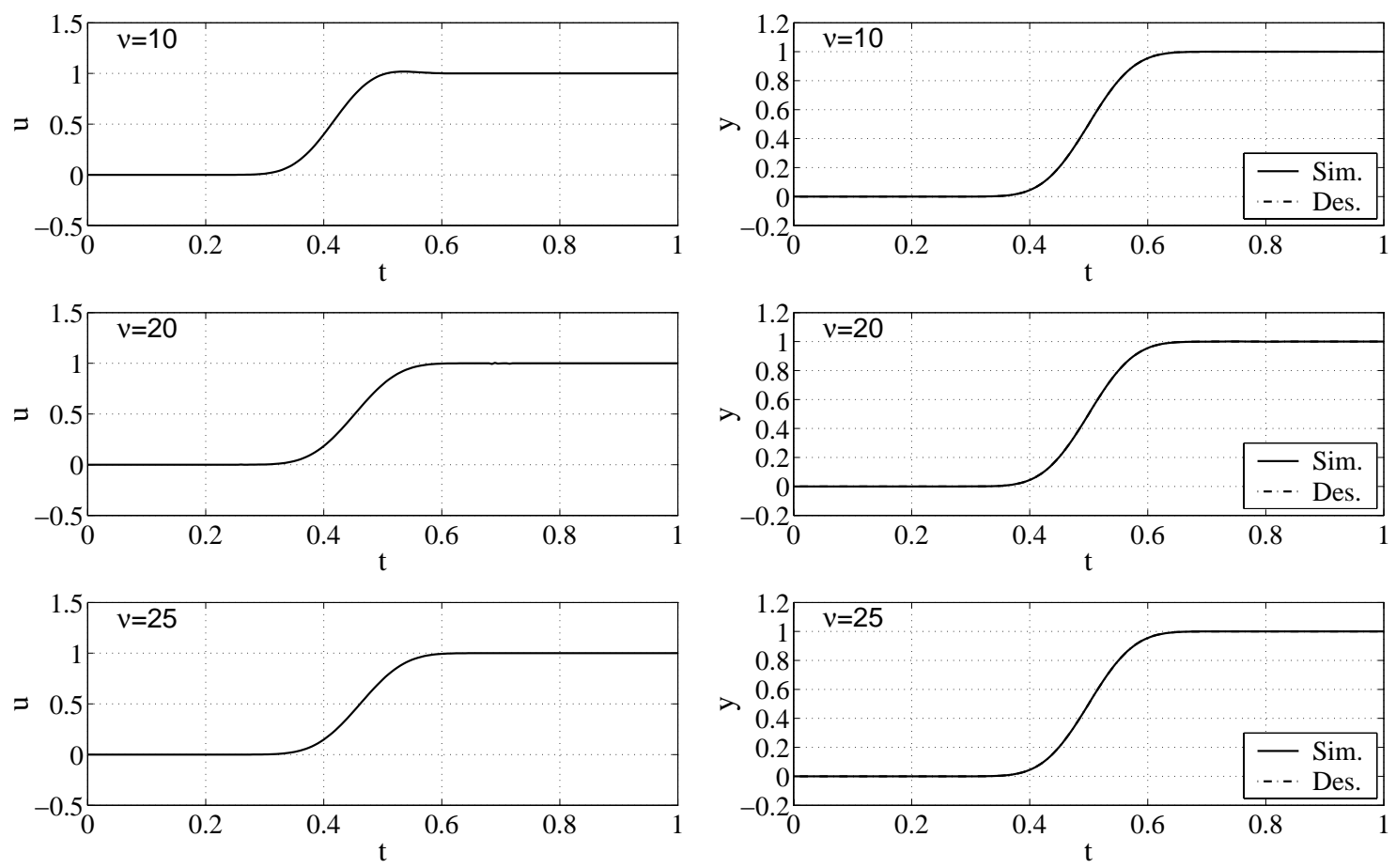

Figure 4.5: Open-loop control of the linear diffusion-convection equation (2.1)-(2.4) using Aitken's algorithm (3.31) for $\lambda=1, \beta=0, p_{1}=0, r_{1}=1, \Psi(x)=0$ with $\nu \in\{10,20,25\}$ varied and desired trajectory $y_{d}(t)=\Phi_{\gamma, T}(t)$ (B.1) of Gevrey order $\alpha=1.5$ for $T=1$. Left: feedforward control $u_{N_{\mathcal{S}}}(t)$ from (2.17); right: output $y(t)=x(0, t)$ compared to desired trajectory $y_{d}(t)$.
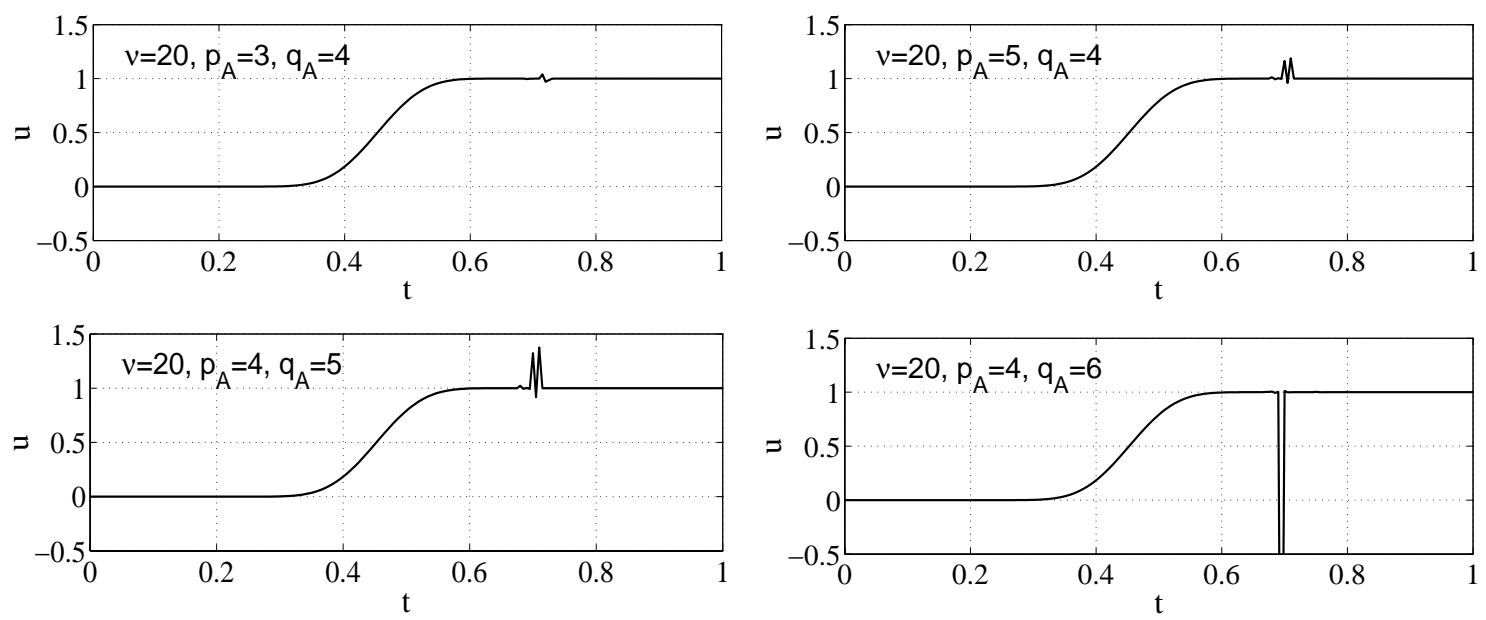

Figure 4.6: Open-loop control from Aitken's algorithm (3.31) for $\lambda=1, \nu=20, \beta=0, p_{1}=0$, $r_{1}=1, \Psi(x)=0$ with summation parameters $p_{\mathcal{A I}}, q_{\mathcal{A I}}$ varied for desired trajectory $y_{d}(t)=\Phi_{\gamma, T}(t)$ (B.1) of Gevrey order $\alpha=1.5$ and $T=1$.

for $q_{\mathcal{A I}}=4$ (top) and varied value of $q_{\mathcal{A I}} \in\{5,6\}$ for $p_{\mathcal{A I}}=4$ (bottom) compared to the 'nominal' parameter set of Figure 4.5, i.e. $p_{\mathcal{A I}}=4$ and $q_{\mathcal{A I}}=4$.

On the other hand, the results for the feedforward control $u_{N_{\mathcal{S}}}(t)$ obtained from the application of Weniger's $\delta_{p_{\delta}}^{\left(q_{\delta}\right)}$ process, illustrate its limited applicability to sum series resulting from formal 

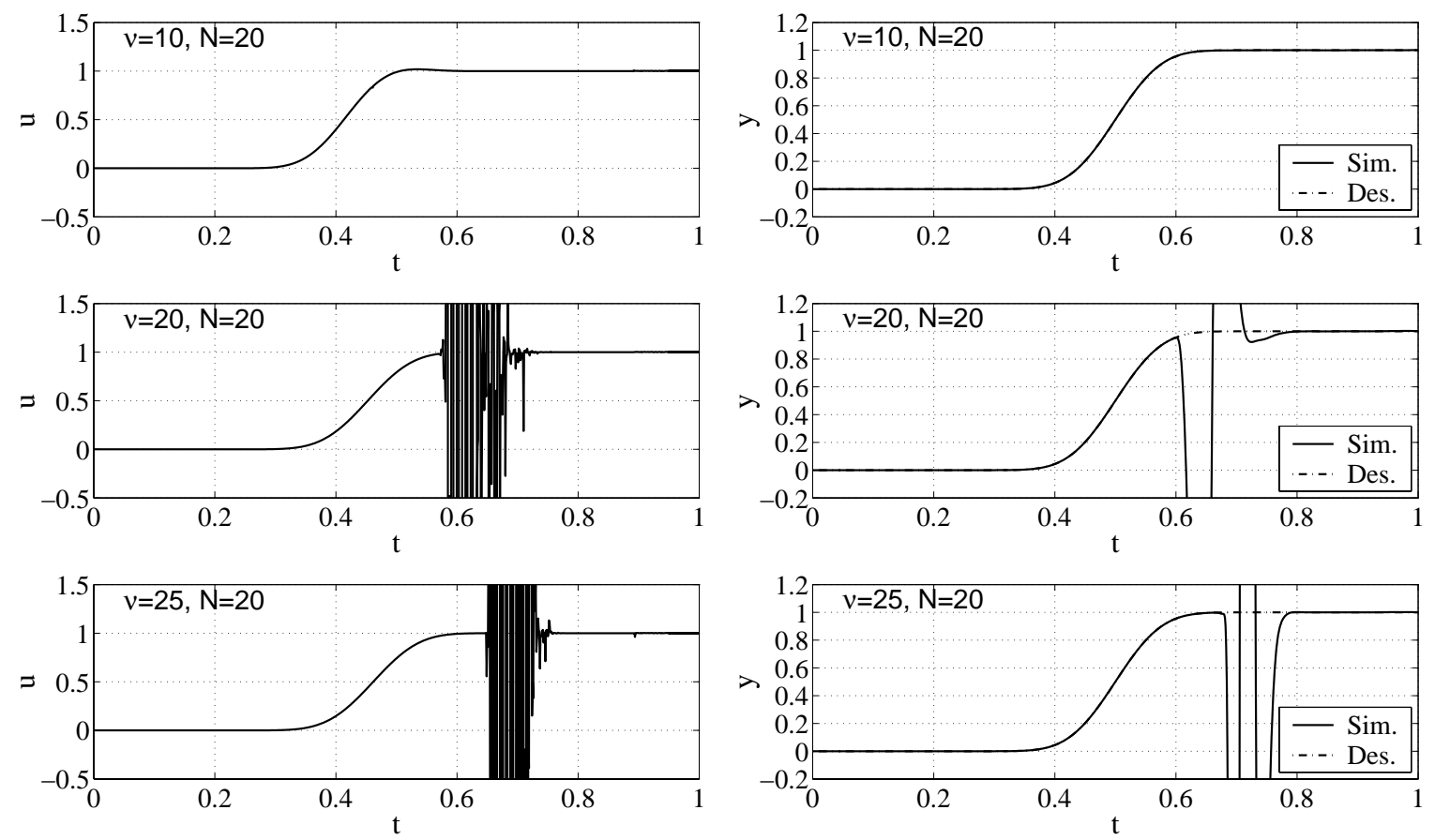

Figure 4.7: Open-loop control of the linear diffusion-convection equation (2.1)-(2.4) using Weniger's $\delta$-algorithm (3.33) for $\lambda=1, \beta=0, p_{1}=0, r_{1}=1, \Psi(x)=0$ with $\nu \in\{10,20,25\}$ varied and desired trajectory $y_{d}(t)=\Phi_{\gamma, T}(t)$ (B.1) of Gevrey order $\alpha=1.5$ for $T=1$. Left: feedforward control $u_{N_{\mathcal{S}}}(t)$ from (2.17); right: output $y(t)=x(0, t)$ compared to desired trajectory $y_{d}(t)$.

solutions of convection dominated DCR-equations. For higher convection rates $\nu>10$, spurious oscillations appear which increase in amplitude and intensity with increasing $\nu$. Detailed parameter studies when varying the parameters $p_{\delta}$ and $\zeta$ of $\delta_{p_{\delta}}^{\left(q_{\delta}\right)}(\zeta)$ with $q_{\delta}=0$ indicate that it is impossible to recover the characteristic behavior of the governing system using $\delta_{p_{\delta}}^{\left(q_{\delta}\right)}(\zeta)$ to approximate the series limit. Note that for increasing $\nu$, the diffusion-convection equation tends to the advection equation, such that the input has to converge to the output $y_{d}(t)$ shifted by $1 / \nu$, i.e. $u_{N_{\mathcal{S}}}(t) \approx y_{d}(t-1 / \nu)$ for $\nu \gg 1$. The inability of Weniger $\delta$-algorithm to sum this convergent series up to its natural sum might be a direct consequence of the non-regularity of this nonlinear generalized sequence transformation as outlined in Section 3.5.

However, application of $\left(N_{\mathcal{S}}, \xi\right)$-approximate $k$-sum $\mathcal{S}_{k}^{\left(N_{\mathcal{S}}, \xi\right)}(3.26)$ with suitably chosen summation parameters $\xi$ and $k$ yields highly accurate results for any considered value of $\nu$. The natural choice of $k=1$ stems from the fact that the series is known to converge, such that only $\xi$ has to be adapted as a function of $\nu$, i.e. $\xi=\xi(\nu)$. Note that due to the verified convergence of the formal solution, accurate results for the feedforward control are also obtained for $k=0$ with values of $\xi \in(0,1)$. In order to illustrate the effect of the increasing convection rate, i.e. the diffusion-convection equation approaches the linear advection equation, the corresponding desired output trajectories $y_{d}(t)$ are compared to the obtained feedforward term $u_{N_{\mathcal{S}}}(t)$ in Figure 4.8, left column. Obviously, the required predictive behavior of $u_{N_{\mathcal{S}}}(t)$ is recovered from the summation procedure. In addition, the wide range applicability of the 

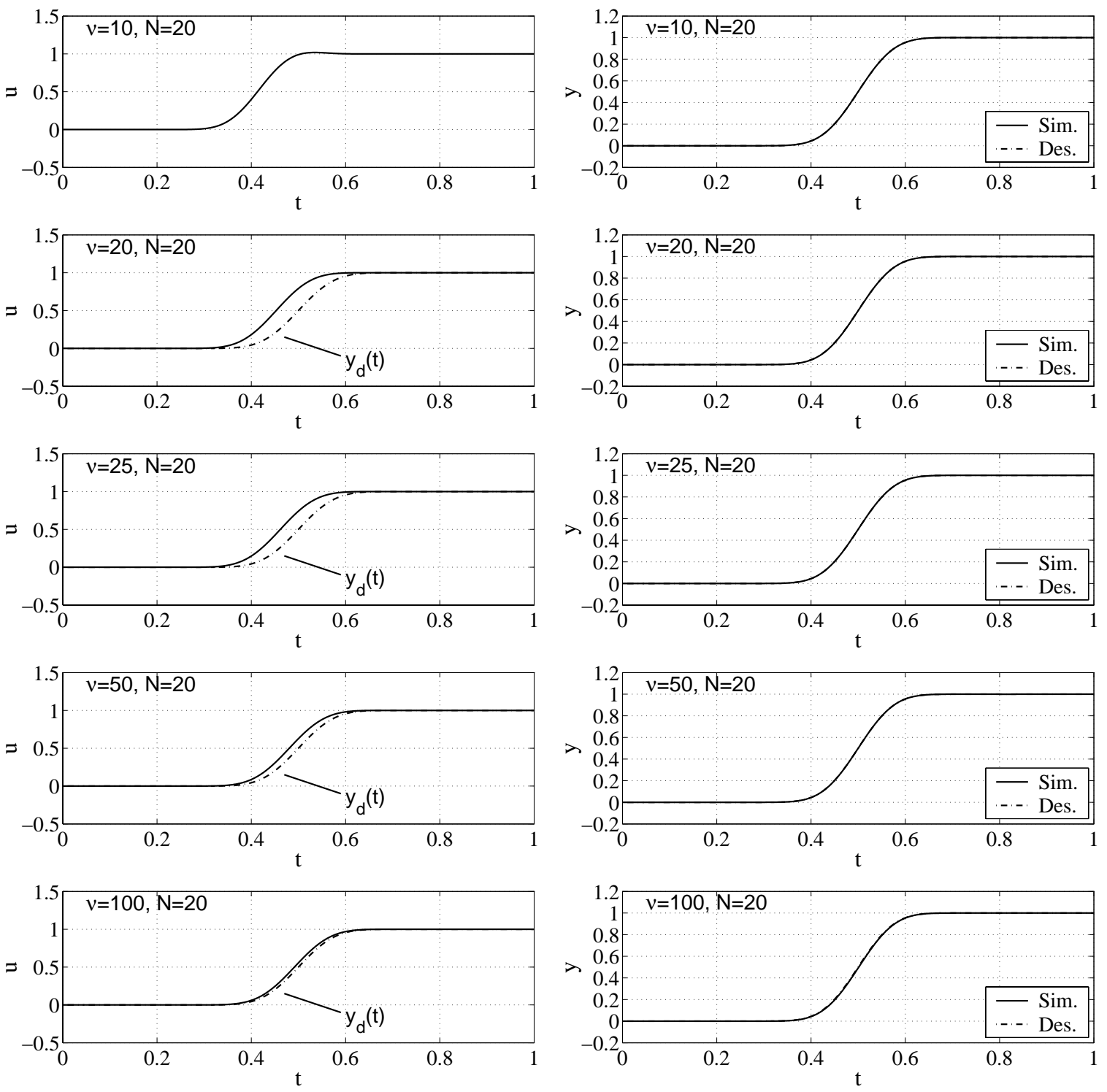

Figure 4.8: Open-loop control of the linear diffusion-convection equation $(2.1)-(2.4)$ using $\left(N_{\mathcal{S}}, \xi\right)-$ approximate $k$-sum (3.26) for $\lambda=1, \beta=0, p_{1}=0, r_{1}=1, \Psi(x)=0$ with $\nu \in\{10,20,25,50,100\}$ varied and $y_{d}(t)=\Phi_{\gamma, T}(t)$ (B.1) of Gevrey order $\alpha=1.5$ for $T=1$. Left: feedforward control $u_{N_{\mathcal{S}}}(t)$ from (2.17) compared to desired trajectory $y_{d}(t)$ to illustrate the effect of higher convection rates; right: output $y(t)=x(0, t)$ compared to desired trajectory $y_{d}(t)$.

$\left(N_{\mathcal{S}}, \xi\right)$-approximate $k$-sum $(3.26)$ is illustrated in the two lower rows of Figure 4.8, where numerical results are depicted for the boundary controlled linear diffusion-convection equation (2.1)-(2.4) with convection parameter $\nu=50$ and $\nu=100$.

\subsubsection{Summary}

The results of this section clearly illustrate the applicability of formal power series in conjunction with summation methods for the feedforward control design for boundary controlled linear 
DCR-systems. It is thereby shown, that depending on the problem under consideration, the studied summation techniques yield significantly differing results. Thereby, the considered approaches provide equally well results for the summation of divergent solutions to the heat equation. However, Weniger's $\delta$-algorithm fails to accelerate convergence of the linear diffusionconvection equation. On the other hand and although successfully applied to divergent series and convergence acceleration, the computational effort for the evaluation of Aitken's iterated algorithm naturally exceeds the effort for the evaluation of the $(N, \xi)$-approximate $k$-sum by several orders of magnitude. Furthermore as is shown for the linear diffusion-convection equation, the iterated algorithm exhibits a high sensitivity with respect the chosen summation parameters $p_{\mathcal{A I}}$ and $q_{\mathcal{A I}}$, with small deviations resulting in large scale effects in the summation quality. Hence in the following, the application of the $(N, \xi)$-approximate $k$-sum is emphasized for feedforward control design for boundary controlled systems of DCR-equations.

\subsection{MIMO DCR-equations with boundary inputs}

In the sequel, summation methods with special focus on the $(N, \xi)$-approximate $k$-summation are applied to determine feedforward control laws for formal power series parameterizable systems of DCR-equations with boundary controls. This provides a systematization of the promising results of the previous section for scalar DCR-equations. Thereby, the broad range applicability of the approach combining formal power series and sophisticated summation methods is confirmed by simulation results for the tubular reactor model of Section 2.2.2.

\subsubsection{Feedforward control design for general DCR-systems}

Based on the promising results for the linear heat and diffusion-convection equation in Sections 4.3.1 and 4.3.2, the considered summation methods are applied to systems of boundary controlled DCR-equations. This generalization is supplemented by an optimization approach to determine the available degrees-of-freedom within the summation techniques.

\subsubsection{Summation of series resulting from DCR-systems}

As illustrated in Section 2.2.1, the general system of DCR-equations (2.39)-(2.42) is formal power series parameterizable if the condition of Theorem 2.4 is satisfied. Hence the formal parameterizations of system state $\boldsymbol{x}(z, t)$ and boundary input $\boldsymbol{u}(t)$ can be determined from Eqns. (2.57) and (2.58), respectively, i.e.

$$
\hat{\boldsymbol{x}}(z, t)=\sum_{n=0}^{\infty}\left[\boldsymbol{\Upsilon}_{2 n}\left(\underline{\boldsymbol{y}}_{n}(t), \underline{\boldsymbol{\zeta}}_{n}(t)\right) z^{2 n}+\boldsymbol{\Upsilon}_{2 n+1}\left(\underline{\boldsymbol{y}}_{n}(t), \underline{\boldsymbol{\zeta}}_{n}(t)\right) z^{2 n+1}\right]=: \sum_{n=0}^{\infty} \hat{\boldsymbol{x}}_{n}(t) z^{n}
$$




$$
\begin{aligned}
\hat{\boldsymbol{u}}(t) & =Q^{-1} \sum_{n=0}^{\infty}\left(\left[R_{1}+2 n P_{1}\right] \mathbf{\Upsilon}_{2 n}\left(\underline{\boldsymbol{y}}_{n}(t), \underline{\boldsymbol{\zeta}}_{n}(t)\right)+\left[R_{1}+(2 n+1) P_{1}\right] \mathbf{\Upsilon}_{2 n+1}\left(\underline{\boldsymbol{y}}_{n}(t), \underline{\boldsymbol{\zeta}}_{n}(t)\right)\right) \\
& =: \sum_{n=0}^{\infty} \hat{\boldsymbol{u}}_{n}(t)
\end{aligned}
$$

with coefficients $\boldsymbol{\Upsilon}_{2 n}\left(\underline{\boldsymbol{y}}_{n}(t), \underline{\boldsymbol{\zeta}}_{n}(t)\right)$ and $\left.\boldsymbol{\Upsilon}_{2 n+1} \underline{\boldsymbol{y}}_{n}(t), \underline{\boldsymbol{\zeta}}_{n}(t)\right), n \in \mathbb{N}_{0}$ defined in (2.54), (2.55) and $\underline{\boldsymbol{y}}_{n}(t), \underline{\boldsymbol{\zeta}}_{n}(t)$ from $(2.56)$.

Let $\mathcal{S}_{V}$ denote a set of linear functional on a space of sequences. Based on the theoretical considerations of Section 3.3 on the summation of power series, it is desired that

$$
\mathcal{S}_{V}(\hat{\boldsymbol{x}}(z, \cdot))=\boldsymbol{x}(z, \cdot)
$$

for $|z|<\rho>1$ since $z \in[0,1]$ by assumption. If the series $\hat{\boldsymbol{x}}(z, t)$ converges uniformly, $\mathcal{S}_{V}$ can be replaced by partial summation $\mathcal{S}_{P}$ or any other regular functional. On the other hand as outlined in Section 4.3, if the series $\hat{\boldsymbol{x}}(z, t)$ diverges, the verification of the existence of a functional satisfying (4.16) is mainly an open question with initial results available for certain Cauchy problems neglecting BCs. Furthermore it is shown in Sections 2.1.3 and 2.2 for nonlinear DCR-equations, that the differential recursions obtained from the formal power series analysis are highly complicated and allow to explicitly determine only a finite number of series coefficients $\hat{\boldsymbol{x}}_{n}(t), n=0,1, \ldots, N$. Nevertheless, the promising numerical results of the previous section indicate, that the application of suitable summation techniques on a finite of number of series coefficients allows to approximate the sum of a given (possibly divergent) series with sufficient accuracy.

Thereby it is shown, that in particular the $(N, \xi)$-approximate $k$-summation introduced in Section 3.4.1 combines a highly accurate and computationally efficient approximation of the sum of the governing slowly converging or diverging series. On the other hand, Aitken's $\Delta^{2}$-formula (3.31) while similarly providing high accuracy, suffers from the high computational effort due to its iterative character. Furthermore, the nonlinear $\delta$-algorithm (3.33) and similarly the family of Levin-transformations are not necessarily able to provide comparable accuracy for the considered scenarios as shown in particular for the linear diffusion-convection equation. Hence in the following, the $(N, \xi)$-approximate $k$-sum $\mathcal{S}_{\boldsymbol{k}}^{N, \boldsymbol{\xi}}$ is chosen as the prototype summation approach to approximate the sum of the parameterized series (4.14), (4.15) resulting from the formal power series analysis of boundary controlled systems of DCR-equations.

Thereby, the summation $\mathcal{S}_{k}^{N, \xi}$ can be applied individually on each element $x_{i}(z, t), i=1, \ldots, K$ of $\boldsymbol{x}(z, t)$ or $u_{i}(t), i=1, \ldots, M$ of $\boldsymbol{u}(t)$, respectively. This additionally allows to introduce separate summation parameters $\xi$ and $k$ for each equation. In summary using matrix notation, the following approximations of the sums (4.14) and (4.15) are obtained

$$
\begin{gathered}
\boldsymbol{x}_{N}(z, t)=V_{\boldsymbol{\xi}, \boldsymbol{k}}^{K} \sum_{n=0}^{N} W_{\boldsymbol{\xi}, \boldsymbol{k}}^{K}(n) \sum_{j=0}^{n} \hat{\boldsymbol{x}}_{j}(t) z^{j}, \\
\boldsymbol{u}_{N}(t)=V_{\boldsymbol{\xi}, \boldsymbol{k}}^{M} \sum_{n=0}^{N} W_{\boldsymbol{\xi}, \boldsymbol{k}}^{M}(n) \sum_{j=0}^{n} \hat{\boldsymbol{u}}_{j}(t)
\end{gathered}
$$


with matrices

$$
\begin{aligned}
V_{\boldsymbol{\xi}, \boldsymbol{k}}^{m} & =\operatorname{diag}\left\{1 / \sum_{n=0}^{N} \frac{\xi_{i}^{n}}{\Gamma\left(1+\frac{n}{k_{i}}\right)}\right\}_{i=1, \ldots, m} \in \mathbb{R}^{m \times m} \\
W_{\boldsymbol{\xi}, \boldsymbol{k}}^{m}(n) & =\operatorname{diag}\left\{\frac{\xi_{i}^{n}}{\Gamma\left(1+\frac{n}{k_{i}}\right)}\right\}_{i=1, \ldots, m} \in \mathbb{R}^{m \times m} .
\end{aligned}
$$

for $m \in\{K, M\}$. Note that in the previous section, the two parameters $\xi$ and $k$ of the $(N, \xi)$-approximate $k$-summation are mainly chosen by knowledge of the growth of the series coefficients and by numerical simulation. On the other hand, for the implementation of (4.19) or (4.20), the number of degrees-of-freedom increases up to $2 K$ or $2 M$, respectively. ${ }^{1}$ Hence, a systematic approach is required to determine the summation parameters $\boldsymbol{\xi}$ and $\boldsymbol{k}$.

\subsubsection{Choice of summation parameters in $(\mathrm{N}, \xi)$-approximate $\mathrm{k}-\mathrm{sum}$}

In order to determine the summation parameters $\boldsymbol{\xi}$ and $\boldsymbol{k}$ of the $(N, \xi)$-approximate $\boldsymbol{k}$-sum, information on the growth of the series coefficients depending on the parameterizing function and its time-derivatives is required. However, this information is hardly available when studying formal solutions to systems of DCR-equations due to the complexity of the governing differential recursions. Therefore, an optimization approach is considered, which is based on the fact that the formal power series analysis is focused on the design of boundary tracking control. Hence, it suffices to determine suitable summation parameters $\boldsymbol{\xi}$ and $\boldsymbol{k}$ for the input parameterization (4.18), whereby the optimization criteria has to be designed to minimize the tracking error (Meurer and Zeitz, 2005).

In the sequel, it is desired to minimize the time-integral over the absolute tracking error on a given sufficiently large time horizon $T$, i.e.

$$
\min _{\boldsymbol{\xi}, \boldsymbol{k}} J\left(\boldsymbol{y}(t), \boldsymbol{y}_{d}(t), \boldsymbol{u}_{d}(t)\right)
$$

where

$$
J\left(\boldsymbol{y}(t), \boldsymbol{y}_{d}(t), \boldsymbol{u}_{d}(t)\right)=\int_{0}^{T}\left[\boldsymbol{y}\left(\tau ; \boldsymbol{u}_{d}(\tau)\right)-\boldsymbol{y}_{d}(t)\right]^{T} \mathcal{W}\left[\boldsymbol{y}\left(\tau ; \boldsymbol{u}_{d}(\tau)\right)-\boldsymbol{y}_{d}(t)\right] d \tau
$$

with the positive definite, symmetric weighting matrix $\mathcal{W} \in \mathbb{R}^{M \times M}$. Here, $\boldsymbol{y}\left(\tau ; \boldsymbol{u}_{d}(\tau)\right)$ denotes the solution of the feedforward controlled DCR-system (2.39)-(2.42) with boundary inputs $\boldsymbol{u}_{d}(\cdot)$ evaluated at time $\tau$. Note that the optimization for the determination of the summation parameters $\boldsymbol{\xi}$ and $\boldsymbol{k}$ can be also performed in closed-loop when combining the feedforward control $\boldsymbol{u}_{d}(t)$ with some feedback control e.g. standard PID-control, for stabilization and/or compensation of model errors and exogenous disturbances. Furthermore, the chosen optimization approach also allows to impose additional constraints, e.g. on the input values.

\footnotetext{
${ }^{1}$ As a further generalization, $N$ could be varied individually for each sum increasing the number of degreesof-freedom by $K$ or $M$, respectively.
} 
In the following, the results of Section 4.4.1 are applied to the feedforward control design for the tubular reactor model of Section 2.2.2. Thereby, the proposed optimization approach is used to determine the summation parameters of the $(N, \xi)$-approximate $k$-sum.

\subsubsection{Feedforward control design for the tubular reactor model}

As illustrated in Section 2.2.2 when studying convergence of the series resulting from the formal power series analysis of the model of a non-isothermal tubular reactor, the available convergence conditions (Lynch and Rudolph, 2000c, 2002; Meurer et al., 2003; Meurer and Zeitz, 2004b) are too restrictive for most physical situations. In particular, the Peclet-numbers are restricted to $P e_{i}<2, i=1,2$, with numbers up to $P e_{1}=200$ and $P e_{2}=100$ arising in applications (Jensen and Ray, 1982). In other words, operation is performed in a highly convective regime, while formal solutions are only available in a highly diffusive region.

Motivated by the promising results obtained from the application of $(N, \xi)$-approximate $k^{-}$ summation for convergence acceleration and summation of divergent series, this technique is applied to feedforward tracking control design for the formal power series parameterizable tubular reactor model (2.61)-(2.68). Therefore, the trajectory (4.5) as depicted in Figure 4.1 is chosen exemplarily to realize a transition between four consecutive operating points. These are determined by the numerical solution of the parameterized BVP (4.3) with $\boldsymbol{y}_{S}^{i}, i=1,2,3,4$ as prescribed by the trajectory $\boldsymbol{y}_{d}(t)$. The corresponding input parameterization follows directly from the substitution of (4.5) into (2.78), i.e.

$$
\begin{aligned}
u_{i, d}(t)= & y_{i, d}(t)+\sum_{n=0}^{\infty}\left[1+\frac{2 n+1}{P e_{i}}\right] \Upsilon_{i, 2 n+1}\left(\boldsymbol{y}_{d}, \ldots, \boldsymbol{y}_{d}^{(n)}\right)+ \\
& \sum_{n=1}^{\infty}\left[1+\frac{2 n}{P e_{i}}\right] \Upsilon_{i, 2 n}\left(\boldsymbol{y}_{d}, \ldots, \boldsymbol{y}_{d}^{(n-1)}, y_{i, d}^{(n)}\right)=: \sum_{n=0}^{\infty} \hat{u}_{i, n}^{d} .
\end{aligned}
$$

Since the complexity of the governing differential recursion (2.72)-(2.75) for the determination the parameterized series coefficients $\Upsilon_{i, n}(\cdot), i=1,2$ prevents a closed-form solution, only a finite number $N$ of addends can be determined using e.g. the algorithm described in Appendix C. Hence in view of numerical evaluation, $\boldsymbol{u}_{d}(t)$ has to be replaced by its $(N, \xi)$-approximate $k$-sum, i.e.

$$
\boldsymbol{u}_{N, d}(t)=\mathcal{S}_{\boldsymbol{k}}^{N, \boldsymbol{\xi}}\left(\sum_{n=0}^{\infty} \hat{\boldsymbol{u}}_{n}^{d}\right)
$$

For the evaluation of the differential recursion, Assumption 2.2 on the nonlinearities has to be justified:

Remark 4.4. For the application of the formal power series approach, the nonlinear source (2.66) has to be approximated following Assumption 2.2. Therefore, similar to Lynch and Rudolph (2000c), a first order Taylor linearization of $\tilde{\varphi}\left(x_{2}\right)=\exp \left(x_{2} /\left(1+x_{2} / \sigma\right)\right)$ around some 
constant reference value $x_{2}^{0}$ can be performed to determine the coefficients $a_{0}$ and $a_{1}$ of (2.70), i.e.

$$
a_{0}=\tilde{\varphi}\left(x_{2}^{0}\right)\left(1-\frac{x_{2}^{0}}{\left(1+\frac{x_{2}^{0}}{\sigma}\right)^{2}}\right), \quad a_{1}=\frac{\tilde{\varphi}\left(x_{2}^{0}\right)-a_{0}}{x_{2}^{0}} .
$$

Alternatively, the linearization can be evaluated along the desired trajectory $y_{2, d}(t)$, which leads to much higher accuracy but time-varying coefficients $a_{0}(t) a_{1}(t)$ and more complex differential recursions. Hence, for any of the following simulations, $a_{0}(t)$ and $a_{1}(t)$ are assumed to be slowly varying, such that $d^{n} a_{i}(t) / d t^{n} \approx 0$ for $n>0, i=1,2$. This has proven to be a good trade-off between accuracy and computational complexity.

For the determination of the summation parameters $\boldsymbol{\xi}=\left(\xi_{1}, \xi_{2}\right)$ and $\boldsymbol{k}=\left(k_{1}, k_{2}\right)$ in $\mathcal{S}_{\boldsymbol{k}}^{N, \boldsymbol{\xi}}$, the optimization approach introduced in the previous section can be applied for given parameter sets of the tubular reactor model - see Appendix D.2 and Table D.1.

The numerical simulations are carried out using a high order semi-discretized version of the DPS (2.61)-(2.68). Note that the feedforward controls are determined in view of Remark 4.4, whereby the simulation model is synthesized using the full nonlinear convection-reaction term $\boldsymbol{\Psi}(\boldsymbol{x}, \partial \boldsymbol{x} / \partial z)$ governed by (2.66). Simulation results for the application of $\mathcal{S}_{\boldsymbol{k}}^{N, \boldsymbol{\xi}}$ with parameters from Table D.1 are depicted in Figures 4.9 and 4.10. Following Jensen and Ray (1982), the chosen parameter sets correspond to an empty tube while $P e_{1}=P e_{2}, L e=1$ (Figure 4.9, (a), (b)) and merge into a packed fixed bed reactor (Figure 4.10) for $P e_{1}=200, P e_{2}=100$, $L e=500$. Note that throughout the literature on control of tubular reactors, usually low and unrealistic $P e$-numbers are employed (among others e.g. by Christofides (2001) or Antoniades and Christofides (2001) as summarized in Panfilov and Sheintuch (2001)), whereby obviously the proposed approach for feedforward control design is applicable for wide parameter ranges. The rather large overshoot in the $y_{2}$ trajectory in Figure 4.10 for the considered severe case with high Peclet- and Lewis-numbers is thereby due to the choice of constant summation parameters $\boldsymbol{\xi}$ and $\boldsymbol{k}$ over the transition interval. It can be shown that an appropriate variation of $\boldsymbol{\xi}$ and $\boldsymbol{k}$ over time significantly reduces the overshoot.

In order to illustrate the superior approximation quality when applying the $(N, \xi)$-approximate $k$-summation to the tubular reactor model, Figure 4.11 shows the feedforward term $u_{1, d}(t)$ obtained from the evaluation of Aitken's algorithm (3.31) with summation parameters $p_{\mathcal{A I}}$ and $q_{\mathcal{A I}}$ varied for the fixed-bed case with $P e_{1}=200, P e_{2}=100$, and $L e=500$. Obviously, spurious oscillations appear, which deteriorate the approximation quality. In addition, the results show the sensitivity of the algorithm to the choice of $p_{\mathcal{A I}}$ and $q_{\mathcal{A I}}$. Furthermore, it should be noted that the necessary computational time to evaluate the (3.31) is of several magnitudes larger than for $\mathcal{S}_{\boldsymbol{k}}^{N, \boldsymbol{\xi}}$, i.e. the quotient $r_{\mathrm{cpu}}:=t_{\mathrm{cpu}}\left(\mathcal{A I}_{p_{\mathcal{A I}}}^{\left(q_{\mathcal{A}}\right)}\right) / t_{\mathrm{cpu}}\left(\mathcal{S}_{k}^{N, \xi}\right)$ with $t_{\mathrm{cpu}}(\cdot)$ the CPU-time necessary for evaluation of the respective summation procedure, is given by $r_{\mathrm{cpu}}=1189$ for $p_{\mathcal{A I}}=3=q_{\mathcal{A I}}$ (Figure 4.11, top) and $r_{\mathrm{cpu}}=43837$ for $p_{\mathcal{A I}}=4, q_{\mathcal{A I}}=5$ (Figure 4.11, bottom). On the other hand, application of Weniger's $\delta$-algorithm provides smooth but inaccurate results for the feedforward control as is illustrated in Figure 4.12. There, the feedforward controls 
(a) $P e_{1}=6, P e_{2}=6, L e=1$
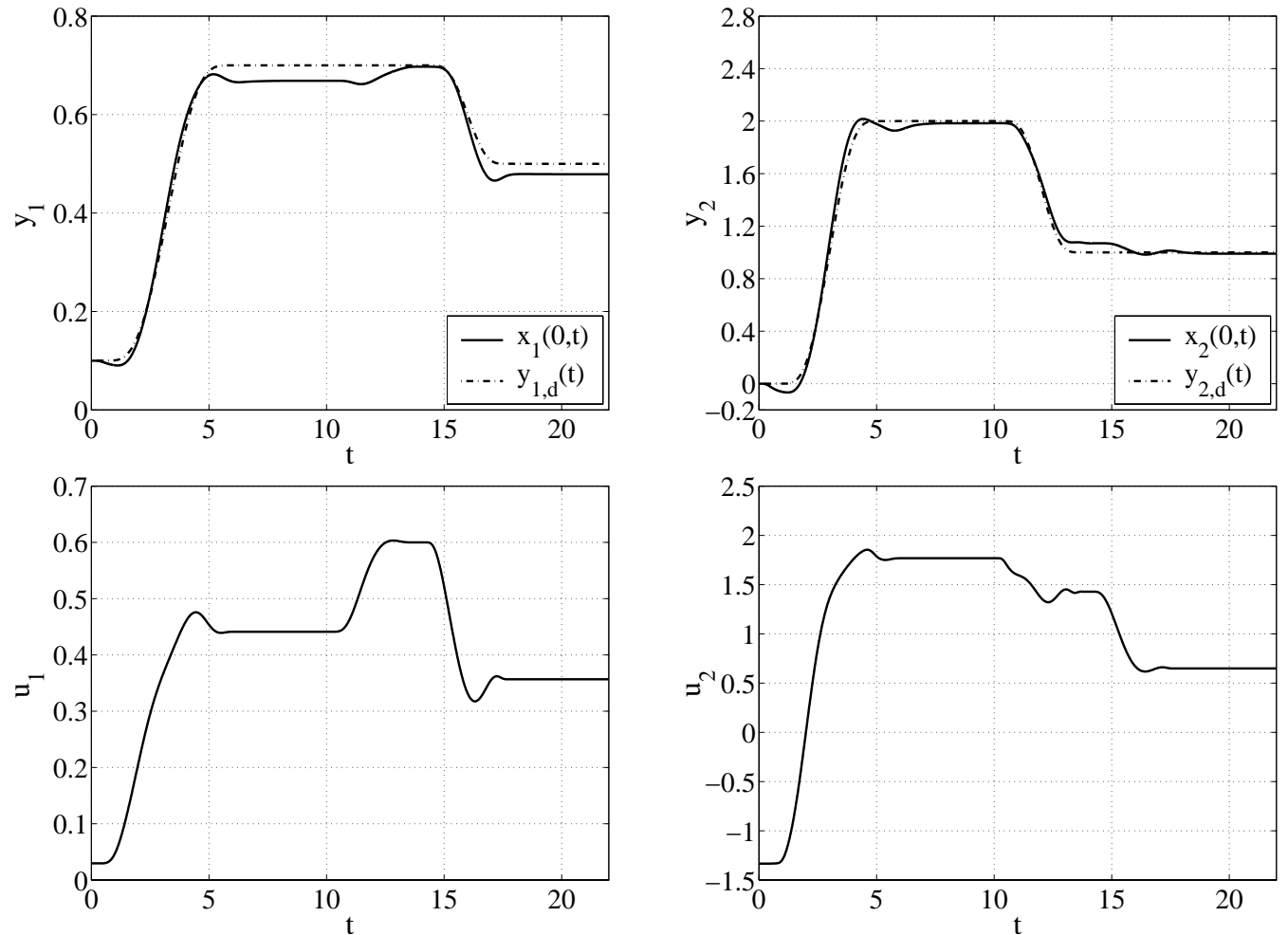

(b) $P e_{1}=10, P e_{2}=10, L e=1$
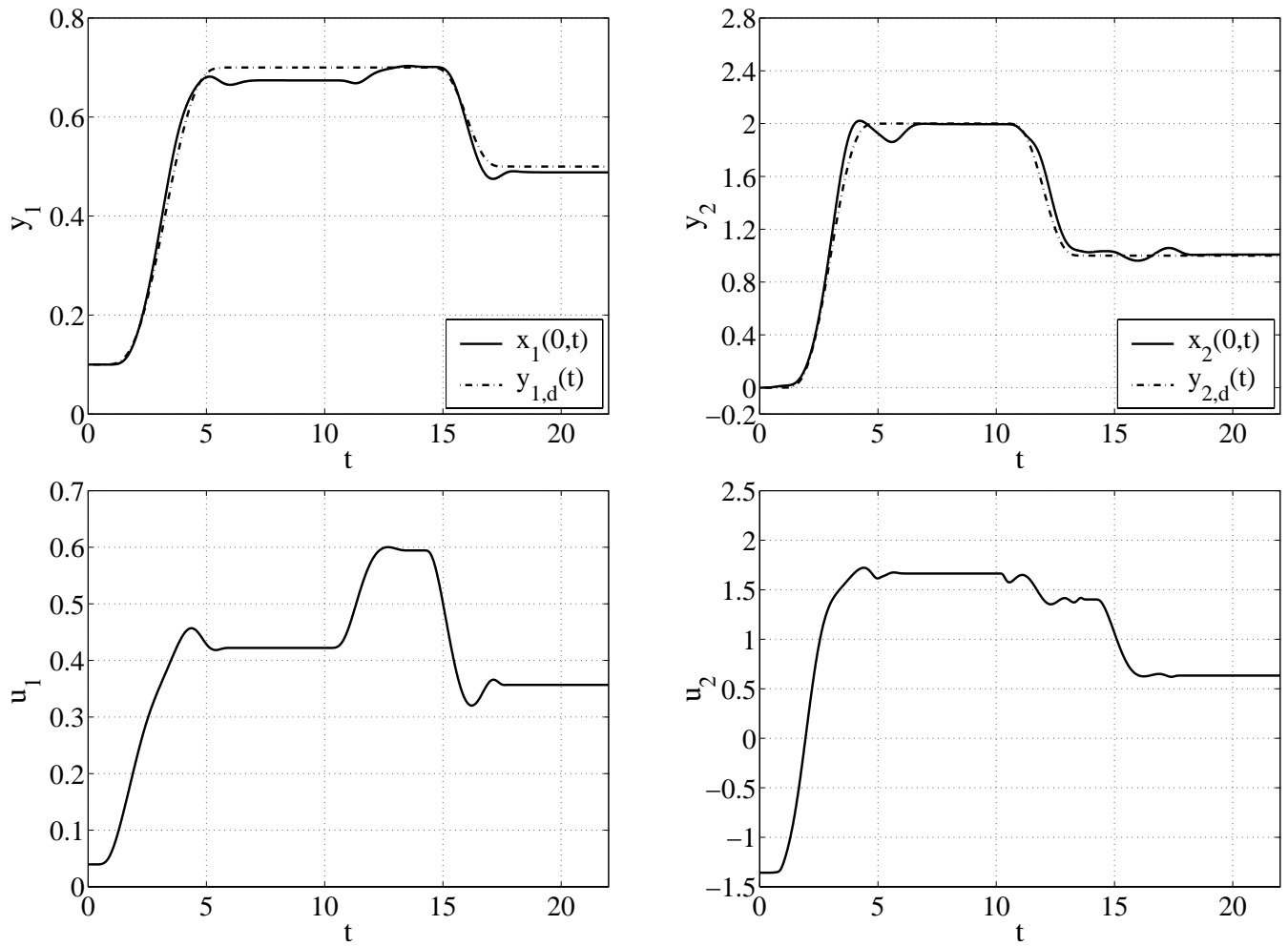

Figure 4.9: Simulation results for feedforward tracking control (4.23) of the tubular reactor model (2.61)-(2.68) with Peclet numbers varied for $L e=1$. Remaining parameters are summarized in Appendix D.2. Top: tracking behavior for $x_{1}(0, t)$ and $x_{2}(0, t)$, bottom: boundary inputs $(2.62)$. 
(c) $P e_{1}=200, P e_{2}=100, L e=500$
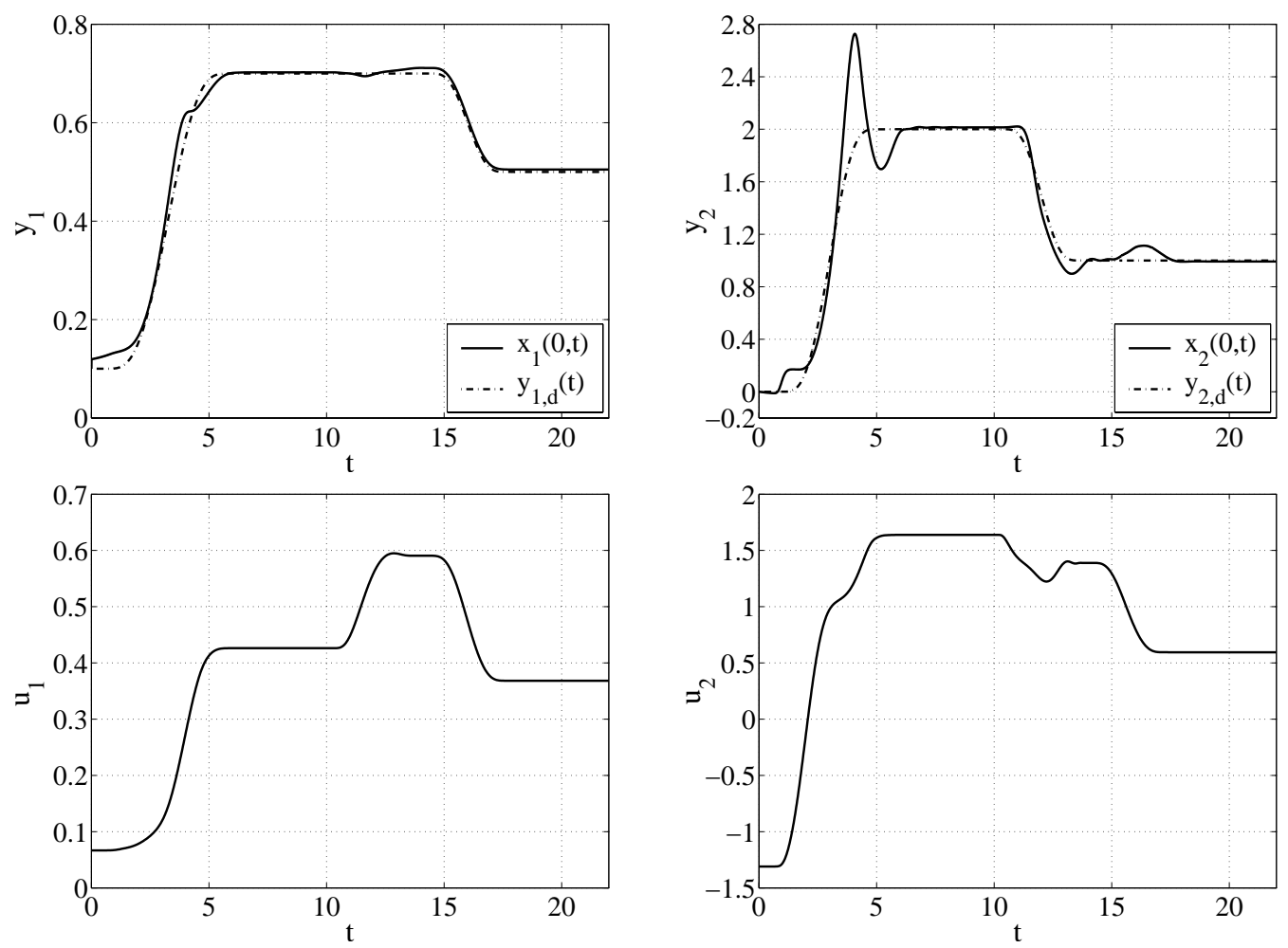

Figure 4.10: Simulation results for feedforward tracking control (4.23) of the tubular reactor model (2.61)-(2.68) with $P e_{1}=200, P e_{2}=100, L e=500$. Remaining parameters are summarized in Appendix D.2. Top: tracking behavior for $x_{1}(0, t)$ and $x_{2}(0, t)$, bottom: boundary inputs $(2.62)$.

obtained from Weniger's $\delta$-algorithm with $p_{\delta}=20, q_{\delta}=0, \zeta=1$ are compared the functions determined from the $(N, \xi)$-approximate $k$-summation. Obviously, the rather large deviations in the control $u_{2}$ are reflected in the output behavior, where hot spots can be identified. Note that different choices of the parameters $p_{\delta}, q_{\delta}, \zeta$ result in a further deterioration of the results.

\subsection{Conclusions}

As illustrated in this section, formal power series in conjunction with advanced summation processes provide a powerful tool for motion planning and feedforward control design for linear and nonlinear sets of DCR-equations with boundary inputs.

Thereby, the introduced $(N, \xi)$-approximate $k$-summation allows to re-cover a meaningful limit from a given possibly slowly converging or diverging series in wide parameter ranges. This is confirmed by the numerical results obtained for the linear heat and diffusion-convection equation and the nonlinear model of a non-isothermal tubular reactor. These examples also illustrate the drawbacks of well-known generalized sequence transformations from quantum physics and quantum chemistry, such as Aitken's $\Delta^{2}$-formula or Weniger's $\delta$-algorithm. Their non-applicability is either founded in the weak accuracy when applied to the parameterized 
series or excessive computational time required for their numerical application.

Due to approximation errors, which either originate from the series truncation or are induced by the approximation of nonlinear terms during formal power series analysis, deviations between desired and actual behavior naturally arise. Hence a stabilizing feedback strategy is required to supplement the feedforward control. Thereby, similarly formal power can be utilized as will be illustrated in the following chapter.
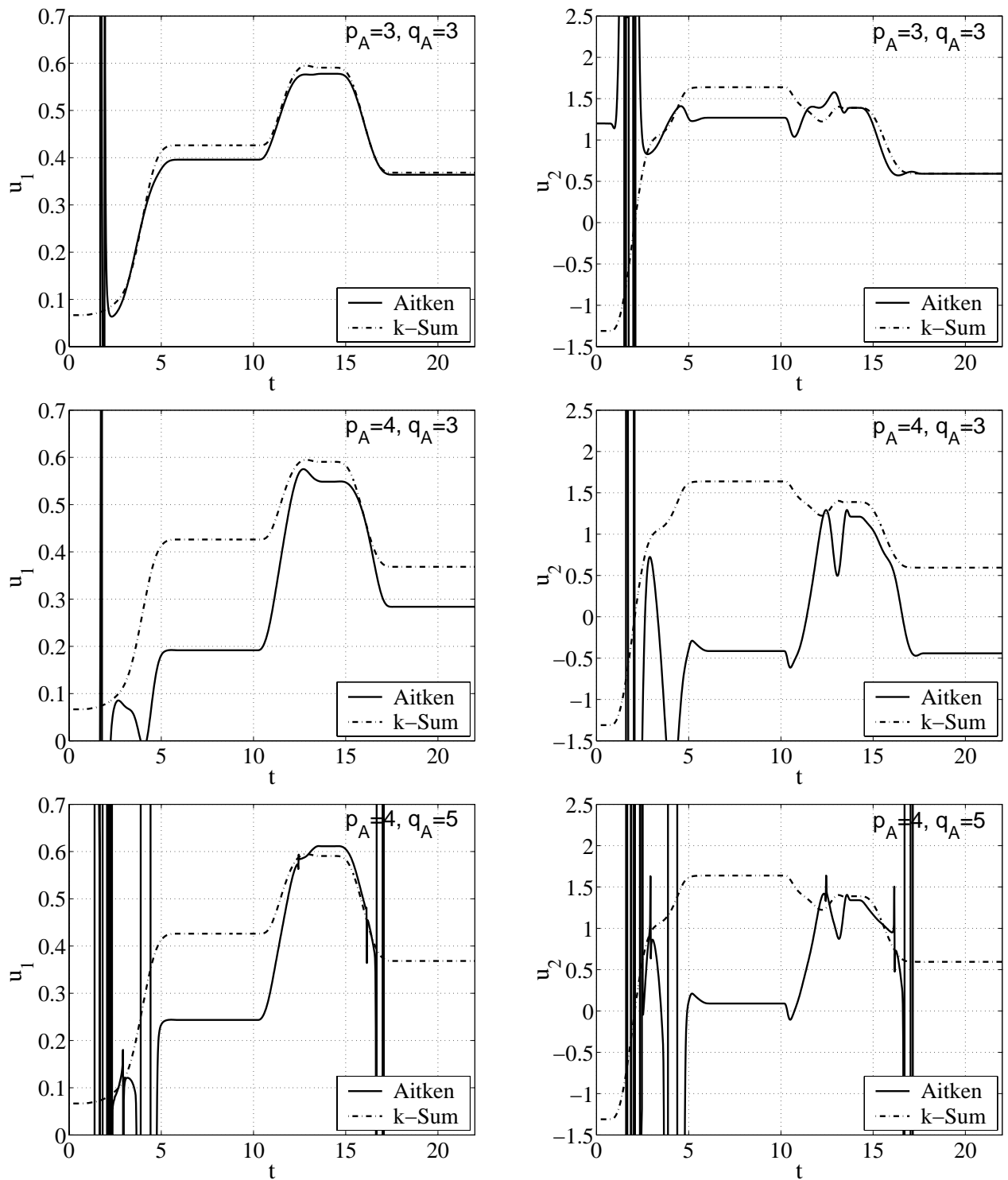

Figure 4.11: Feedforward tracking control (4.22) for the tubular reactor model (2.61)-(2.68) with $P e_{1}=200, P e_{2}=100, L e=500$, determined by application of Aitken's algorithm (3.31). For comparison purposes, the feedforward controls of Figure 4.10 for the $(N, \xi)$-approximate $k$-sum are depicted additionally. Remaining parameters are summarized in Appendix D.2. From top to bottom: variation of the parameters $p_{\mathcal{A I}}$ and $q_{\mathcal{A I}}$ in (3.31). 

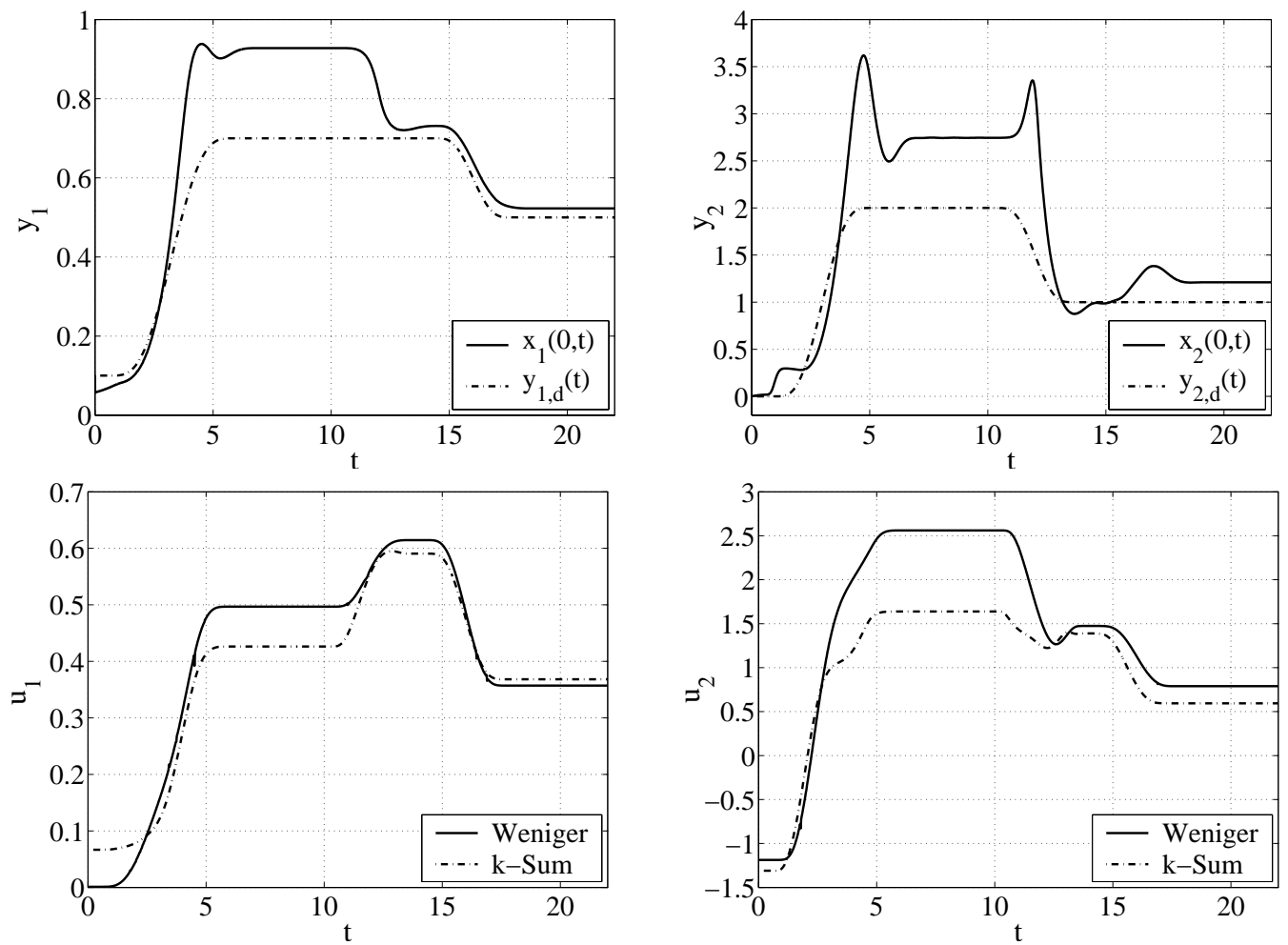

Figure 4.12: Feedforward tracking control (4.22) for the tubular reactor model (2.61)-(2.68) with $P e_{1}=200, P e_{2}=100, L e=500$, determined by application of Weniger's $\delta$-algorithm with $p_{\delta}=20$, $q_{\delta}=0, \zeta=1$. For comparison purposes, the feedforward controls of Figure 4.10 for the $(N, \xi)-$ approximate $k$-sum are depicted additionally. Remaining parameters are summarized in Appendix D.2. Top: tracking behavior for $x_{1}(0, t)$ and $x_{2}(0, t)$, bottom: boundary inputs $(2.62)$. 


\section{Chapter 5}

\section{Flatness-based feedback boundary tracking control of DCR-systems}

As illustrated in the previous chapter on feedforward control design for boundary controlled DCR-systems, formal power series in conjunction with appropriate summation methods provide a widely applicable approach allowing high tracking performance. Nevertheless except for the nominal case with stable and perfectly known plant model, the feedforward part has to be supplemented by a stabilizing feedback part in order to compensate for model errors or exogenous disturbances and to robustly track the desired trajectory.

For nonlinear finite-dimensional systems, the property of differential flatness has proven to provide a uniform methodology for asymptotic tracking control design - see e.g. Fliess et al. (1995); Martin et al. (1997); Rothfuß et al. (1996); Rothfuß (1997); Rudolph (2000a, 2003a) and the references therein. Due to the vast number of successfully applied and implemented flatness-based methods in finite-dimensions, an extension of this design approach to infinitedimensional DPS would be desirable.

Therefore in the following, formal power series and summation methods are utilized to feedback tracking control design for boundary controlled nonlinear DCR-systems. This approach is based on the introduced notion of formal power series parameterizability in Section 2.3, whose re-interpretation allows to determine a finite-dimensional and inherently differentially flat approximation of the governing DPS as a nonlinear system of ODEs in the coefficients of the formal power series. For this finite-dimensional approximation, standard flatness-based methods can be utilized for asymptotic tracking control design with observer. Thereby, the estimated data from the observer can be directly applied for spatial profile estimation and monitoring throughout the transition process.

\subsection{Scalar DCR-equations with boundary input}

In this section, the proposed approach for feedback tracking control using formal power series is applied to scalar boundary controlled DCR-systems. For the illustration of the main ideas, 
detailed results are derived for the linear heat equation, while results for the nonlinear case are embedded in the more general considerations in the second part of the chapter.

\subsubsection{Feedback tracking control design for the linear heat equation}

Starting from the results of Section 2.1.1 on the formal parameterization of the linear heat equation (2.1)-(2.4) with boundary control for $\nu=0$ and $\Psi(x) \equiv 0$, formal power series are applied to determine a finite-dimensional approximation of the governing DPS model. The proposed approach is based on the system's inverse (2.13) relating the boundary input $u(t)$ and the parameterizing function $y(t)$.

Hence recall the parameterization in the operator domain (2.18), which can be equivalently expressed ${ }^{1}$ in the time domain as

$$
\frac{d \hat{x}_{2 n}}{d t}=(2 n+2)(2 n+1) \lambda \hat{x}_{2 n+2}+\beta \hat{x}_{2 n}, \quad \forall n \in \mathbb{N}_{0},
$$

since $\hat{x}_{2 n+1}=0, \forall n \in \mathbb{N}_{0}$ due to (2.19). In Section 2.1.1, this expression is interpreted as a differential recursion for the coefficients of the series $\hat{x}(z, t)=\sum_{n=0}^{\infty} \hat{x}_{n}(t) z^{n}$ by imposing $\hat{x}_{0}(t)=$ $y(t)=x(0, t)$. As illustrated, the resulting series $\hat{x}(z, t)$ converges uniformly for functions $y(t) \in G_{M, R, \alpha}\left(\mathbb{R}^{+}\right)$with $\alpha \leq 2$, i.e. functions being Gevrey of order $\alpha \leq 2$. Thus it suffices to consider only a finite number of series coefficients $\hat{x}_{n}(t)$ to approximate the sum $\hat{x}(z, t)$.

In the sequel, this result is exploited to determine a finite-dimensional system approximation suitable for feedback control design.

\subsubsection{Finite-dimensional design model via formal power series and summation methods}

The main idea is based on the re-interpretation of the differential recursion (5.1) as a system of ODEs in the coefficients $\hat{x}_{2 n}, n \geq 0$ of the power series under the formal assumption of uniform convergence (Meurer, 2002). As a result, the summation index $n$ can be limited to $n \in\{0,1, \ldots, N-1\}$ for a certain integer $N>1$. Hence, (5.1) reads as follows

$$
\begin{aligned}
\frac{d \hat{x}_{0}}{d t} & =2 \lambda \hat{x}_{2}+\beta \hat{x}_{0} \\
\frac{d \hat{x}_{2}}{d t} \quad & =12 \lambda \hat{x}_{4}+\beta \hat{x}_{2} \\
\quad & \\
\frac{d \hat{x}_{2 N-4}}{d t} & =(2 N-2)(2 N-3) \lambda \hat{x}_{2 N-2}+\beta \hat{x}_{2 N-4} \\
\frac{d \hat{x}_{2 N-2}}{d t} & =(2 N)(2 N-1) \lambda \hat{x}_{2 N}+\beta \hat{x}_{2 N-2}
\end{aligned}
$$

\footnotetext{
${ }^{1}$ The same result can be determined from the differential recursion (2.24) for the linear diffusion-convection equation by substituting $\nu=0$.
} 
with the remaining unknown coefficient $\hat{x}_{2 N}$. Obviously, the choice of $\hat{x}_{0}=y$ directly yields the coefficient parameterization (2.21) for $n \in\{0,1, \ldots, N-1\}$. On the other hand, evaluate the inhomogeneous BC (2.4) using the series $\hat{x}(z, t)$, i.e.

$$
\hat{u}=\sum_{n=0}^{\infty}\left[p_{1}(n+1) \hat{x}_{n+1}+r_{1} \hat{x}_{n}\right]=: \sum_{n=0}^{\infty} \hat{u}_{n} .
$$

By truncating this series at $n=2 N-1$ due the convergence assumption, i.e.

$$
\hat{u} \approx \sum_{n=0}^{2 N-1} \hat{u}_{n}=r_{1} \hat{x}_{0}+\sum_{n=0}^{2 N-1}\left(n p_{1}+r_{1}\right) \hat{x}_{n}+2 N p_{1} \hat{x}_{2 N},
$$

it is possible to substitute the coefficient $\hat{x}_{2 N}$ in $(5.2){ }^{2}$ Alternatively, the generally rather restrictive convergence requirement can be formally replaced by the less constraining assumption of $k$-summability as discussed in Chapters 3 and 4 . Hence instead of truncating (5.3) at $n=2 N-1$, its $(2 N-1, \xi)$-approximate $k$-sum $\mathcal{S}_{k}^{2 N-1, \xi}$ defined in (3.25) can be introduced, such that

$$
\hat{u} \approx \frac{\sum_{n=0}^{2 N-1}\left(\sum_{j=0}^{n} \hat{u}_{j}\right) \frac{\xi^{n}}{\Gamma\left(1+\frac{n}{k}\right)}}{\sum_{n=0}^{2 N-1} \frac{\xi^{n}}{\Gamma\left(1+\frac{n}{k}\right)}}=v_{\xi, k} \sum_{n=0}^{2 N-1} w_{\xi, k}(n) \sum_{j=0}^{n} \hat{u}_{j}
$$

where

$$
v_{\xi, k}=\frac{1}{\sum_{n=0}^{2 N-1} \frac{\xi^{n}}{\Gamma\left(1+\frac{n}{k}\right)}}, \quad w_{\xi, k}(n)=\frac{\xi^{n}}{\Gamma\left(1+\frac{n}{k}\right)} .
$$

In view of $\hat{x}_{2 n+1}=0, \forall n \in \mathbb{N}_{0}$ and (5.4), solving (5.5) for $\hat{x}_{2 N}$ provides after some intermediate calculations that

$$
\hat{x}_{2 N}=-\sum_{n=0}^{N-1} a_{\xi, k}^{N}(2 n) \hat{x}_{2 n}+b_{\xi, k}^{N} \hat{u}
$$

with

$$
\begin{aligned}
a_{\xi, k}^{N}(2 n) & =\frac{1}{2 N p_{1}}\left\{2 n p_{1} w_{\xi, k}^{-1}(2 N-1) w_{\xi, k}(2 n-1)+\left(1+w_{\xi, k}^{-1}(2 N-1) \sum_{j=2 n}^{2 N-2} w_{\xi, k}(j)\right)\left[2 n p_{1}+r_{1}\right]\right\} \\
b_{\xi, k}^{N} & =\frac{1}{2 N p_{1}} w_{\xi, k}^{-1}(2 N-1) v_{\xi, k}^{-1} .
\end{aligned}
$$

The following remark summarizes the results for the special case of $p_{1}=0$ :

\footnotetext{
${ }^{2}$ By taking into account the term $r_{1} \hat{x}_{2 N}$ from the evaluation of the series (5.3) up to the limit $2 N$, the input $u$ can be similarly introduced for the following computations. Nevertheless, the above choice has proven to provide the most suitable results.
} 
Remark 5.5. If $p_{1}=0$ in the $B C$ (2.4), i.e. $r_{1} x(1, t)=u(t)$, the ansatz (5.4) has to be redefined in terms of $\hat{u} \approx \sum_{n=0}^{2 N} \hat{u}_{n}$ with $\hat{u}_{n}:=r_{1} \hat{x}_{n}$. After introducing the $(2 N, \xi)$-approximate $k$-sum, (5.6) is similarly recovered after some intermediate computations, whereby

$$
a_{\xi, k}^{N}(2 n)=1+w_{\xi, k}^{-1}(2 N) \sum_{j=2 n}^{2 N-1} w_{\xi, k}(j), \quad b_{\xi, k}^{N}=\frac{1}{r_{1}} w_{\xi, k}^{-1}(2 N) v_{\xi, k}^{-1} .
$$

In either case, substitution of (5.6) into (5.2) results in the $N$-dimensional dynamical SISO system (5.7)-(5.8) with the state $\hat{\boldsymbol{x}}_{2 N-2}^{\prime}=\left[x_{0}, x_{2}, \ldots, x_{2 N-2}\right]^{T}$ as described by Eqns. (5.7)(5.9) in Figure 5.1. Thereby, the corresponding expression (5.9) of the parameterizing function $y(t)=x(0, t)=\hat{x}_{0}(t)$ is provided additionally. Using the introduced abbreviations, the derived finite-dimensional approximation can be expressed in state space notation of a linear SISO system

$$
\begin{aligned}
& \dot{\hat{\boldsymbol{x}}}_{2 N-2}^{\prime}=A \hat{\boldsymbol{x}}_{2 N-2}^{\prime}+\boldsymbol{b} \hat{u}, \quad t>0 \\
& \hat{\boldsymbol{x}}_{2 N-2}^{\prime}(0)=\hat{\boldsymbol{x}}_{2 N-2,0}^{\prime} \in \mathbb{R}^{N} \\
& y \quad=\boldsymbol{c}^{T} \hat{\boldsymbol{x}}_{2 N-2}^{\prime} .
\end{aligned}
$$

In order to illustrate the ability to approximate the dynamics of the governing linear heat equation (2.1)-(2.4) with $\nu=0$ and $\Psi(x)=0$ using the ODEs (5.10), consider Figure 5.2. There, the spectrum $\iota(L)$ of the operator

$$
L h=\frac{d^{2} h}{d z^{2}}+\beta h
$$

with domain

$$
\mathcal{D}(L)=\left\{h \in \boldsymbol{L}_{2}(0,1): h, \frac{d h}{d z} \text { abs. cont., } \frac{d^{2} h}{d z^{2}} \in \boldsymbol{L}_{2}(0,1), \frac{d h}{d z}(0)=0=p_{1} \frac{d h}{d z}(1)+r_{1} h(1)\right\}
$$

is depicted and compared to the numerically determined eigenvalue distribution of the dynamics matrix $A$ of (5.7) or respectively (5.10), when varying the summation parameters $\xi$ and $k$ as well as the dimension $N$. Thereby, the parameters are chosen consistently with respect to Sections 2.1 .1 and 4.3.1, i.e. $\lambda=1, \beta=0, r_{1}=1, p_{1}=0$. For this choice, the spectrum of the operator $L$ can be easily determined as $\iota(L)=\left\{-\left(\frac{2 i-1}{2} \pi\right)^{2}\right\}_{i \in \mathbb{N}}$. Due to $p_{1}=0$, the results of Remark 5.5 are considered for evaluation.

Obviously, there exist sets of summation parameters $(\xi, k)$, such that for given $N$, the fundamental dynamics characterized by the slow modes is approximated with sufficient accuracy. In particular, the first modes corresponding to $\iota_{i}, i=1,2,3$ are recovered exactly. Nevertheless, residual modes appear due to the ill-posedness of the inverse system (Isakov, 1998). It should be pointed out that the eigenvalues of the system matrix $A$ of the design model for increasing $\xi$ in the $k$-summation ansatz (5.5), tend to the eigenvalues of the system one would obtain from 


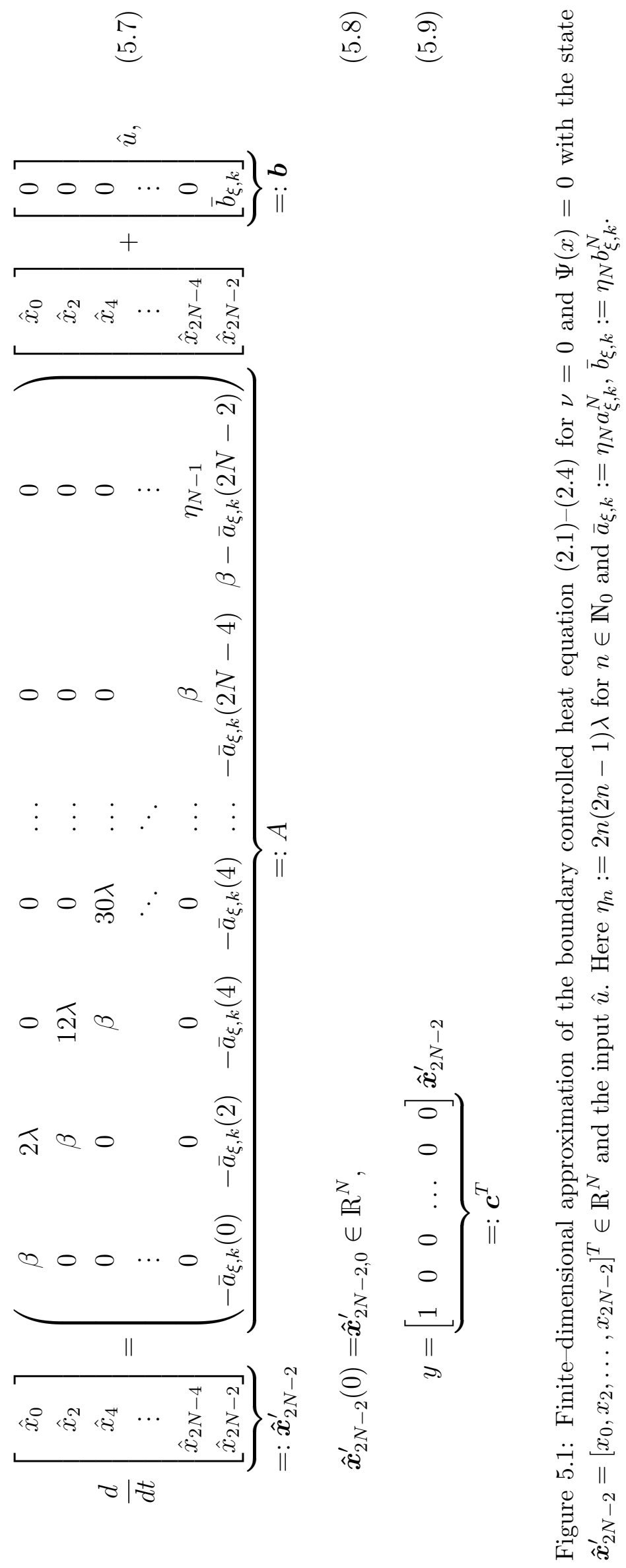


partial summation (5.4) to determine $\hat{x}_{2 N}$. This can be easily clarified since

$$
\begin{aligned}
\frac{\sum_{n=0}^{2 N-1} \overbrace{\left(\sum_{j=0}^{n} \hat{u}_{j}\right)}^{=: \hat{u}_{n}^{\prime}} \frac{\xi^{n}}{\Gamma\left(1+\frac{n}{k}\right)}}{\sum_{n=0}^{2 N-1 \frac{\xi^{n}}{\Gamma\left(1+\frac{n}{k}\right)}}}= & \frac{\hat{u}_{0}^{\prime}}{\sum_{n=0}^{2 N-1} \frac{\xi^{n}}{\Gamma\left(1+\frac{n}{k}\right)}}+\frac{\hat{u}_{1}^{\prime} \frac{\xi}{\Gamma\left(1+\frac{1}{k}\right)}}{\sum_{n=0}^{2 N-1} \frac{\xi^{n}}{\Gamma\left(1+\frac{n}{k}\right)}}+\ldots+\frac{\hat{u}_{2 N-1}^{\prime} \frac{\xi^{2 N-1}}{\Gamma\left(1+\frac{2 N-1}{k}\right)}}{\sum_{n=0}^{2 N-1} \frac{\xi^{n}}{\Gamma\left(1+\frac{n}{k}\right)}} \\
& \approx \frac{\hat{u}_{2 N-1}^{\prime} \frac{\xi^{2 N-1}}{\Gamma\left(1+\frac{2 N-1}{k}\right)}}{\sum_{n=0}^{2 N-1} \frac{\xi^{n}}{\Gamma\left(1+\frac{n}{k}\right)}} \rightarrow \hat{u}_{2 N-1}^{\prime}=\sum_{j=0}^{2 N-1} \hat{u}_{j} \quad \text { for } \xi \gg 1 .
\end{aligned}
$$

As illustrated in Figure 5.2, residual modes in the right-half complex plane are obtained for large $\xi$ when using partial summation to derive the design model. This indicates that the summation parameters have to be chosen carefully in order to avoid unphysical modes. If this is ensured, tracking control can be designed based on the system (5.7)-(5.9) or (5.10)-(5.12), respectively.

\subsubsection{Tracking control with observer}

Due to the structure of the linear system (5.10)-(5.11), which in view of (5.12) can be interpreted as a generalized linear controller normal form, asymptotically stabilizing tracking control can be designed using e.g. standard eigenvalue assignment (Lunze, 2005).

For the considered design model (5.10)-(5.12), a state feedback of the following form is proposed

$$
\begin{aligned}
b_{\xi, k}^{N} \hat{u}= & -\underbrace{\boldsymbol{h}^{T} \hat{\boldsymbol{x}}_{2 N-2}^{\prime}}_{\text {compensation }}-\underbrace{\boldsymbol{s}^{T} \hat{\boldsymbol{x}}_{2 N-2}^{\prime}}_{\text {stabilization }}-\underbrace{\frac{p_{0}}{(2 N-2) ! \lambda^{N-1}} \int_{0}^{t}\left(y(\tau)-y_{d}(\tau)\right) d \tau}_{\text {integral part }}+ \\
& \underbrace{\frac{1}{(2 N-2) ! \lambda^{N-1}}\left(y_{d}^{(N)}(t)+\sum_{n=0}^{N-1} p_{n} y_{d}^{(n)}(t)\right)}_{\text {feedforward control }} .
\end{aligned}
$$

The control law consists of a compensation part, a stabilization part by eigenvalue assignment, an integral part to achieve zero steady state error, and a feedforward part to prescribe the motion along the desired trajectory $t \mapsto y_{d}(t)$ for the output $y(t)$. Thereby, the gains are given by $\boldsymbol{h}^{T}=\left[h_{0}, \ldots, h_{N-1}\right]$ with $h_{n}=\bar{h}_{n}$ for $n=0,1, \ldots, N-1$ and $h_{N-1}=\bar{h}_{N-1}+\beta$ where

$$
\bar{h}_{n}=-\left(a_{\xi, k}^{\bar{N}}(2 n)+\frac{\sum_{j=n-1}^{N-2} g_{j} \bar{g}_{j+1, n}}{(2 N-2) ! \lambda^{N-1}}\right)
$$

as well as $\boldsymbol{s}^{T}=\left[s_{0}, \ldots, s_{N-1}\right]$ with

$$
s_{n}=\sum_{j=n}^{N-1} p_{j+1} \bar{g}_{j, n}
$$



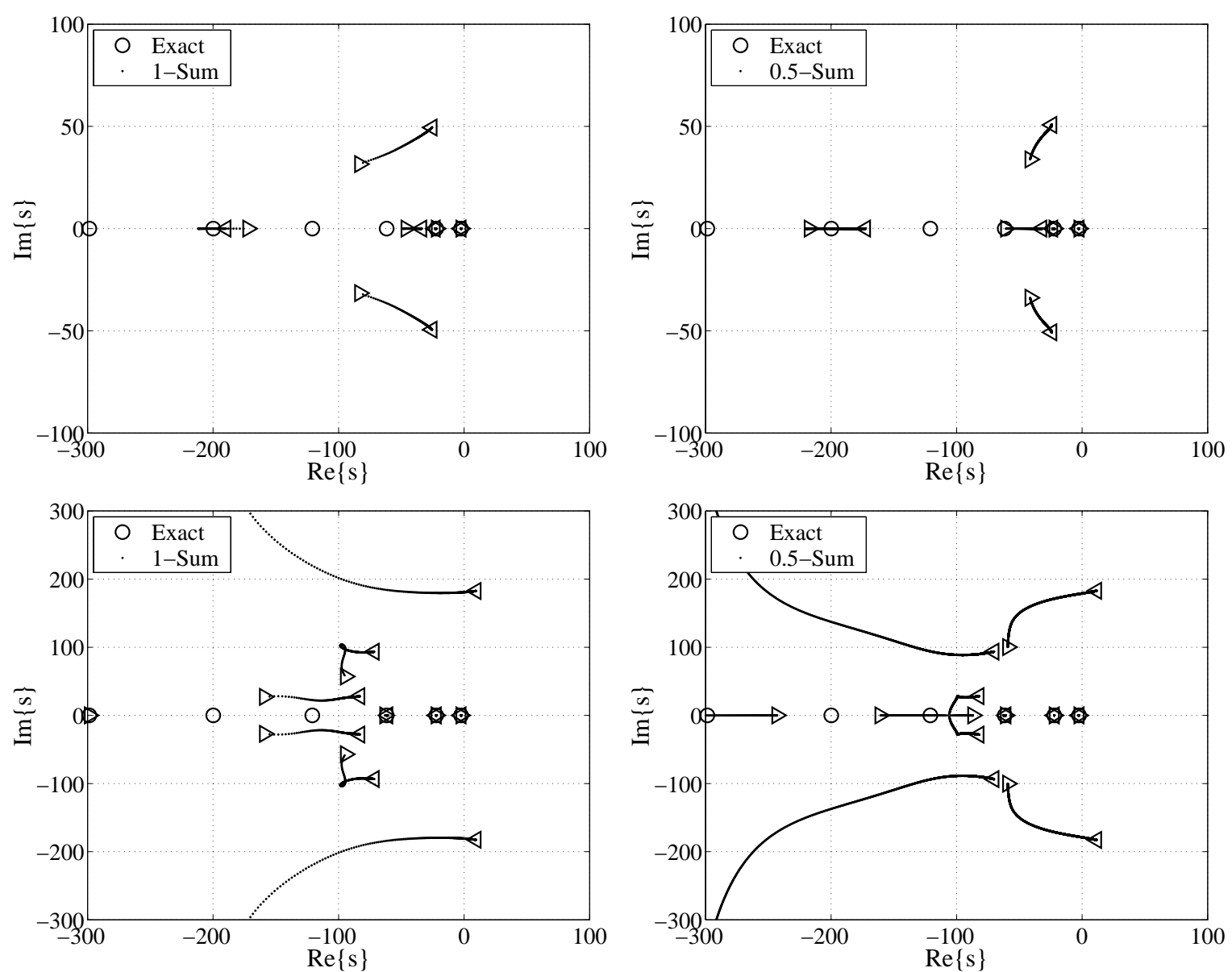

Figure 5.2: Eigenvalue distribution in the complex $s$-domain using $\mathcal{S}_{k}^{2 N, \xi}$ with $N=6$ (top) for $\xi \in[10,40], k=1$ (top,left); $\xi \in[500,4000], k=\frac{1}{2}$ (top,right) and $N=10$ (bottom) for $\xi \in[15,50]$, $k=1$ (bottom,left); $\xi \in[1000,4000], k=\frac{1}{2}$ (bottom,right). Here $\circ$ denotes the spectrum of the operator (5.13), $\triangleright$ and $\triangleleft$ correspond to the eigenvalues for the respective first and last $\xi$ of the given intervals.

for

$$
\bar{g}_{n, j}=\left(\begin{array}{c}
n \\
j
\end{array}\right)(2 j) ! \lambda^{j} \beta^{n-j}, \quad g_{n}=\left\{\begin{array}{ll}
0, & n<0, \\
\left(\begin{array}{c}
N-1 \\
n
\end{array}\right)(-\beta)^{N-1-n}, & n \geq 0,
\end{array} \quad n, j=0,1, \ldots, N-1 .\right.
$$

Note that the proposed tracking control can be directly determined from the property that $y$ from (5.12) denotes a flat output for the system (5.10) (Fliess et al., 1995; Rothfuß, 1997). In particular, the state and input parameterizations directly correspond to the finite-dimensional equivalents of (2.14) and (2.13), respectively, which illustrates that the proposed design model represents indeed an approximation of the inverse DPS.

Application of the state feedback (5.15) to the linear control system (5.10) provides the characteristic polynomial of the closed-loop system as

$$
\varrho^{N+1}+p_{N+1} \varrho^{N}+\ldots+p_{1} \varrho+p_{0}=\prod_{j=0}^{N}\left(\varrho-\varrho_{j}\right)
$$


whose $N+1$ degrees-of-freedom $p_{j}, j=0, \ldots, N$ can be determined by eigenvalue assignment $\varrho_{j}, j=0, \ldots, N$. Note that the characteristic polynomial is of order $N+1$ due to the additional integral part for robustness.

Obviously, full state information is necessary for the implementation of the chosen state feedback (5.15), such that an observer is required to estimate the non-measured states. For its design based on the linear finite-dimensional control system (5.10), (5.11), it is assumed that $y=\hat{x}_{0}=x(0, t)$ of $(5.12)$ is the measured quantity. Consequently, a linear Luenberger observer

$$
\dot{\hat{\boldsymbol{x}}}_{2 N-2}^{\prime}=A \tilde{\hat{\boldsymbol{x}}}_{2 N-2}^{\prime}+\boldsymbol{b} \tilde{\hat{u}}+\boldsymbol{l}\left(y-\boldsymbol{c}^{T} \tilde{\hat{\boldsymbol{x}}}_{2 N-2}^{\prime}\right), \quad \tilde{\hat{\boldsymbol{x}}}_{2 N-2}^{\prime}(0)=\tilde{\hat{\boldsymbol{x}}}_{2 N-2,0}^{\prime}
$$

can be designed with the constant gain vector $\boldsymbol{l} \in \mathbb{R}^{N}$, input $\tilde{\hat{u}}$ from (5.15) with $\hat{\boldsymbol{x}}_{2 N-2}^{\prime}$ replaced by the estimated states $\tilde{\hat{\boldsymbol{x}}}_{2 N-2}^{\prime}$, and suitable initial conditions $\tilde{\hat{\boldsymbol{x}}}_{2 N-2,0}^{\prime}$. Thereby it is assumed, that the characteristic polynomial of the observer error dynamics is Hurwitz with dynamical parameters $q_{j}, j=0,1, \ldots, N-1$ determined by appropriate eigenvalue assignment

$$
\tilde{\varrho}^{N}+q_{N} \tilde{\varrho}^{N-1}+\ldots+q_{1} \tilde{\varrho}+q_{0}=\prod_{j=0}^{N-1}\left(\tilde{\varrho}-\tilde{\varrho}_{j}\right) .
$$

The results of this section provide a control/observer scheme, which stabilizes and robustifies the derived design model (5.10)-(5.11) along the desired trajectory $y_{d}(t)$. Nevertheless, this does not directly ensure stability of the closed-loop system with respect to the infinite-dimensional linear heat equation.

\subsubsection{Stability of the tracking control scheme}

In practice, finite-dimensional control synthesis for the stabilization of DPS is typically based on reduced-order models. Thereby the question arises whether the derived control has the desired effect when applied to the governing infinite-dimensional system (Balas, 1982a,b). This in particular comprises the question of spillover due to unmodeled dynamics in the finitedimensional control (and observer) design model (Balas, 1978). For a comprehensive review of results on stability and stabilizability for DPS, the reader is referred e.g. to Curtain and Zwart (1995) or Luo et al. (1999).

In view of tracking control for the linear heat equation, it is necessary to determine the eigenvalue location of the combined system consisting of the derived feedback/observer scheme (5.15)-(5.18) based on the finite-dimensional design model (5.10)-(5.11) and the governing DPS (2.1)-(2.4) with $\nu=0, \Psi(x)=0$. Thereby, the combined system can be studied as an abstract boundary control problem (Fattorini, 1968; Curtain and Zwart, 1995) with the state $\check{x}(t)=\check{x}(\cdot, t)=x(\cdot, t)-b(\cdot) u(t)$ with $b(z)=z^{2} /\left(2 p_{1}+r_{1}\right)$ with $\check{x}(t) \in \boldsymbol{L}_{2}(0,1)$ and $u(t) \in \mathbb{R}$

$$
\dot{\check{x}}(t)=L \check{x}(t)+\left[\lambda b^{\prime \prime}(z)+\beta b(z)\right] u(t)-b(z) \dot{u}(t), \quad \check{x}(0)=x_{0}-b(z) u_{0}
$$

with operator $L$ on the domain $\mathcal{D}(L)$ defined in (5.13), (5.14). Since this abstract approach complicates analysis due to the appearing time-derivative of the input, in the sequel the 
spectral representation of the system is considered by introducing modal coordinates $x_{i}^{*}:=$ $\left\langle x(z, t), \psi_{i}(z)\right\rangle_{\boldsymbol{L}_{2}(0,1)}, i \in \mathbb{N}$. Here, $\psi_{i}(z)$ denotes the $i$-th eigenfunctions of the operator $L$ on $\mathcal{D}(L)$. Since the set of eigenfunctions $\left\{\psi_{i}(z)\right\}_{i \in \mathbb{N}}$ forms an ortho-normal basis in $\boldsymbol{L}_{2}(0,1)$, application of Green's theorem provides the modal representation of the boundary controlled linear heat equation

$$
\begin{aligned}
\dot{\boldsymbol{x}}^{*}(t) & =A^{*} \boldsymbol{x}^{*}(t)+\boldsymbol{b}^{*} u(t), \quad t>0 \\
\boldsymbol{x}^{*}(0) & =\boldsymbol{x}_{0}^{*} \\
y & =\boldsymbol{c}^{*, T} \boldsymbol{x}^{*}
\end{aligned}
$$

with the modal state $\boldsymbol{x}^{*}(t)=\left[x_{1}^{*}(t), x_{2}^{*}(t), \ldots\right]^{T}$. Here, $A^{*}=\operatorname{diag}\left\{-\gamma_{i}\right\}_{i \in \mathbb{N}}$ is a diagonal matrix with disjoint ordered eigenvalues $\gamma_{i} \in \iota(L)$ where $\gamma_{1}>\gamma_{2}>\ldots, b_{i}^{*}=\lambda / p_{1} \psi_{i}(1)$, and $c_{i}^{*}=\psi_{i}(0)$ for $i \in \mathbb{N}$. Note for $p_{1}=0$ it follows that $b_{i}^{*}=-\lambda / r_{1} \psi_{i}^{\prime}(1)$. In order to study stability and robustness of the closed-loop, the desired trajectory is assigned as $y_{d}(t) \equiv 0$, such that the combined system (5.19), (5.15), (5.17) follows as

$$
\frac{d}{d t}\left[\begin{array}{c}
y \\
\tilde{\hat{\boldsymbol{x}}}_{2 N-2}^{\prime} \\
\boldsymbol{x}^{*}
\end{array}\right]=\underbrace{\left(\begin{array}{c|c|c}
0 & \boldsymbol{c}^{T} & 0 \\
\hline-\boldsymbol{b} \frac{p_{0}}{(2 N-2) ! \lambda^{N-1}} & A-\boldsymbol{b}\left(\boldsymbol{h}^{T}+\boldsymbol{s}^{T}\right)-\boldsymbol{l} \boldsymbol{c}^{T} & \boldsymbol{l} \boldsymbol{c}^{* T} \\
\hline-\boldsymbol{b}^{*} \frac{p_{0}}{(2 N-2) ! \lambda^{N-1}} & -\boldsymbol{b}^{*}\left(\boldsymbol{h}^{T}+\boldsymbol{s}^{T}\right) & A^{*}
\end{array}\right)}_{=: \mathbb{A}}\left[\begin{array}{c}
y \\
\tilde{\hat{\boldsymbol{x}}}_{2 N-2}^{\prime} \\
\boldsymbol{x}^{*}
\end{array}\right] .
$$

Note that the first differential equation is due to the $I$-part in (5.15), which is added for robustness. Let $\iota(\mathbb{A})$ denote the spectrum of $\mathbb{A}$, and let

$$
v:=\max \left\{\Re \iota^{*}: \iota^{*} \in \iota(\mathbb{A})\right\} .
$$

denote the largest of the real parts of the eigenvalues of A. Stability of the combined system requires that $v<0$, whereby robustness requires a certain stability margin, i.e. $v<-\mu, \mu>0$. Curtain (1985) provides a priori estimates for the linear DPS if the reduced order design model is based on modal reduction. However as pointed out in this reference, it is hard to draw conclusions about the effect of an arbitrary compensator design based on a reduced model on the eigenvalues of the combined infinite-dimensional system.

Therefore in the following, the infinite-dimensional matrix $\mathbb{A}$ is projected into a finitedimensional linear space reflecting the dominating dynamics by considering the first $n^{*} \in \mathbb{N}$, $n^{*} \gg 1$ modes in the modal representation (5.19)-(5.21). This approximation is satisfied since the higher modes of $\mathbb{A}$ are much harder to excite and decay faster than $\exp \left(-\gamma_{n^{*}} t\right)$. Furthermore, it is possible to numerically determine an upper bound on the eigenvalues of the combined system by semi-discretizing the governing PDE in space using finite-differences (FD). The resulting system of ODEs exhibits a structure similar to (5.19)-(5.22) with modal states replaced by the values of $x(z, t)$ at the local grid points, matrix $A^{*}$ being in tri-diagonal form, and vectors $\boldsymbol{b}^{*}, \boldsymbol{c}^{*, T}$ adopted respectively. Hence the combined system can be similarly transferred into the structure (5.22). Thereby it can be illustrated that the eigenvalues of 


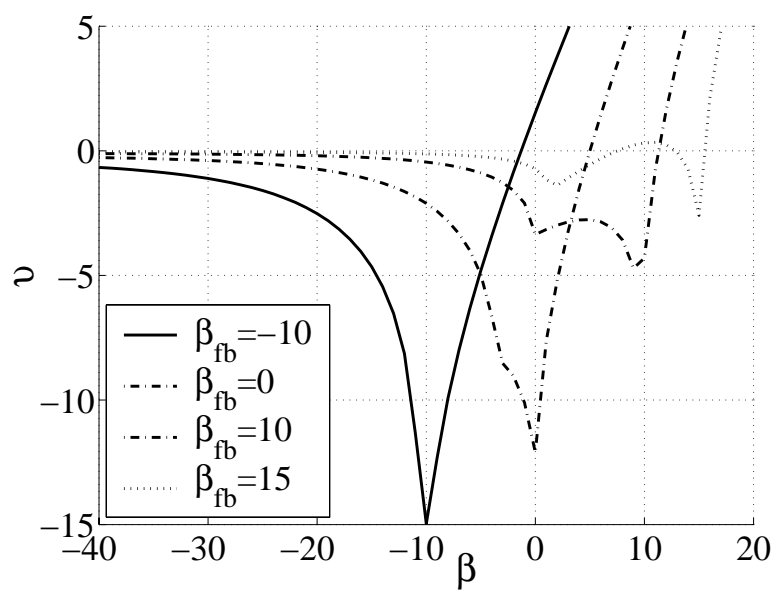

Figure 5.3: Maximal real part $v$ (5.23) of the eigenvalues of $\mathbb{A}$ in (5.22) for $n^{*}=250, \lambda=1, p_{1}=0$, $r_{1}=1$, when varying the system parameter $\beta$ in $\mathbb{A}$ for feedback control with observer (5.15), (5.17) set-up for the respective $\beta_{\mathrm{fb}}$. The remaining parameters are summarized in Table 5.1.

an FD-discretized linear PDE converge from above to the eigenvalues of the governing PDE. Hence a conservative estimate on the dominating eigenvalues of $\mathbb{A}$ can be determined, as is carried out in the following.

In order to study stability and robustness of the combined system (5.22), consider Figure 5.3. There the values of $v$ given by (5.23) are depicted over a variation of the parameter $\beta$ in the PDE or $A^{*}$, respectively, and feedback control with observer (5.15), (5.17) set-up for the indicated fixed values of $\beta_{\mathrm{fb}}$. The remaining system parameters $\lambda=1, p_{1}=0, r_{1}=1$ and number $n^{*}=250$ of considered modes are kept constant. For this choice of parameters, the spectrum of the operator $L$ on domain $\mathcal{D}(L)$ can be determined as $\iota(L)=\left\{\beta-\left(\frac{2 i-1}{2} \pi\right)^{2}\right\}_{i \in \mathbb{N}}$, such that depending on the value of $\beta$ instability occurs. Assigned eigenvalues (5.16) and (5.18), as well as the values of $\xi$ and $k$ in the summation approach are summarized in Table 5.1. Obviously, stability and certain robustness can be ensured as can be seen from the determined curves over $\beta$. Note that although the assigned eigenvalues $\varrho_{i}, i=0,1, \ldots, N$ (5.16) and $\tilde{\varrho}_{j}, j=0,1, \ldots, N-1$ are chosen rather large, the elements of the resulting gains $\boldsymbol{h}^{T}+\boldsymbol{s}^{T}$ and $\boldsymbol{l}$ remain within reasonable intervals due to their interaction with system and summation parameters.

Table 5.1: Parameters for design model (5.10)-(5.12) and assigned eigenvalues for feedback (5.15), (5.16) for $i=0,1, \ldots, N$ and observer (5.17), (5.18) for $j=0,1, \ldots, N-1$.

\begin{tabular}{l|l|l|l|l|}
\hline$\beta_{f b}$ & $\varrho_{i}$ & $\varrho_{j}$ & $\xi$ & $k$ \\
\hline \hline-10 & -35 & $-50-10(j-1)$ & 21.5 & 1 \\
\hline 0 & -35 & $-40-10(j-1)$ & 21.5 & 1 \\
\hline 10 & -25 & $-30-10(j-1)$ & 21.5 & 1 \\
\hline 15 & -25 & $-30-10(j-1)$ & 21.5 & 1 \\
\hline
\end{tabular}



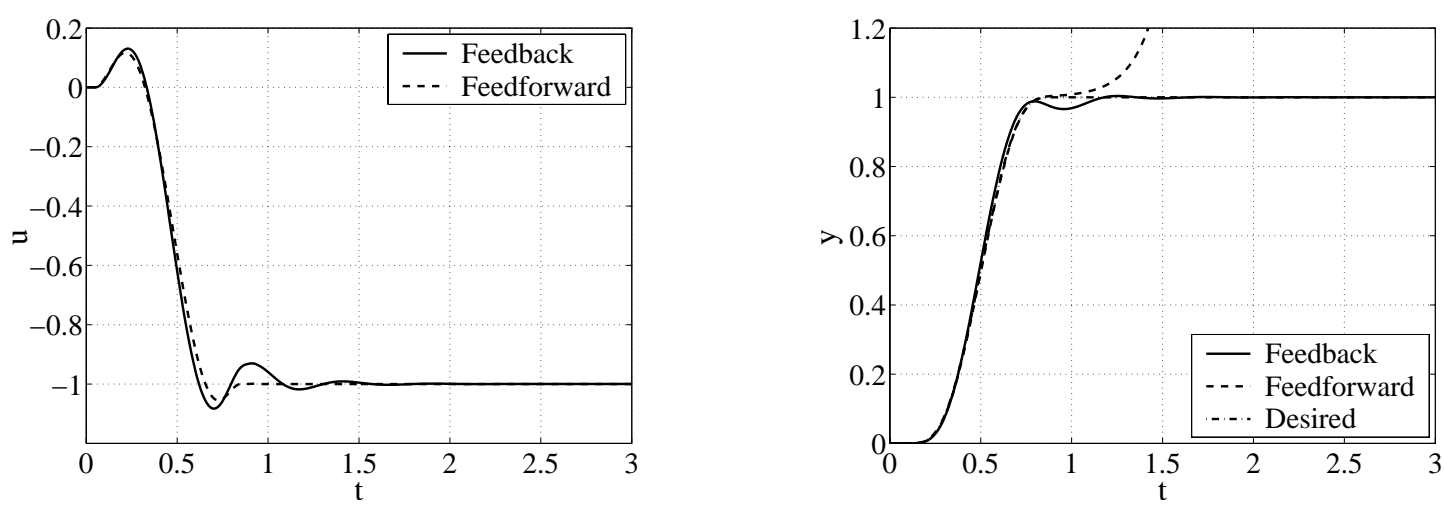

Figure 5.4: Simulation results of input $u(t)$ and output $y(t)$ for feedback tracking control with observer (5.15)-(5.18) for the unstable linear heat equation (2.1)-(2.4) with $\lambda=1, \nu=0, \beta=10, \Psi(x)=0$, $r_{1}=1$, and $p_{1}=0$.

\subsubsection{Simulation results}

For numerical simulation, the boundary controlled linear heat equation (2.1)-(2.4) for $\nu=0$ and $\Psi(x)=0$ is simulated 'in-the-loop', i.e. a sampling time $\Delta t_{\text {sample }}=0.005$ (dimensionless) is explicitly taken into account. Hence, the controls are assumed constant for each simulation time period $t_{\mathrm{sim}^{i+1}}-t_{\mathrm{sim}^{i}}=\Delta t_{\text {sample }}$ and measurements for the evaluation of the observer are only available at discrete instances of time $t_{\mathrm{sim}^{i+1}}$.

Simulation results for the derived tracking control with observer (5.15)-(5.18) are depicted in Figure (5.4) when applied to a MOL discretized model of the linear heat equation. The desired trajectory is assigned as $y_{d}(t)=\Phi_{\gamma, T}(t)$ defined by Eqns. (B.1), (B.2) of the Appendix B with $T=\gamma=1$. System parameters are chosen as $\lambda=0, \beta=10, p_{1}=0, r_{1}=0$, i.e. spectrum $\iota(L)=\{0.075,-0.122,-0.516, \ldots\}$. The design parameters follow from Table 5.1 (3rd row), i.e. $\beta_{\mathrm{fb}}=\beta$. As can be clearly deduced from the simulation results, accurate tracking behavior is obtained.

\subsubsection{Nonlinear scalar DCR-equations}

Starting from the promising results obtained for the linear heat equation in the previous section, the underlying idea of approximating the governing infinite-dimensional DPS by means of differential equations for the series coefficients of the formal power series can be directly extended to the linear diffusion-convection equation and scalar DCR-equations with polynomial nonlinearities as considered in Sections 2.1.2 and 2.1.3. Although the required computations resemble those for the heat equation, their complexity increases. Hence instead of summarizing the results for feedback tracking control design with observer for nonlinear boundary controlled scalar DCR-systems, general results for systems of boundary controlled DCR-equations are derived below, which naturally contain the scalar results. Considering the analysis of scalar 
DCR-systems within the above context, the interested reader is referred to Meurer and Zeitz (2003b, 2004a).

\subsection{MIMO DCR-equations with boundary inputs}

In the sequel, the flatness-based tracking control design approach with observer and spatial profile estimation is illustrated for general boundary controlled DCR-systems introduced in Section 2.2. This provides a generalization and systematization of the results of the previous Section 5.1, where scalar DCR-systems with single boundary input are studied within the proposed tracking control design concept.

In the second part of the section, the derived results are applied to the tubular reactor model considered in Sections 2.2.2 and 4.4.2 in order to design robust tracking control. The control performance is illustrated in several simulation scenarios.

\subsubsection{Feedback tracking control design for general DCR-systems}

It is shown in Section 5.1.1 by studying the linear heat equation with scalar boundary input, that it is possible to determine a finite-dimensional control and observer design model in a generalized controller normal form based on a re-interpretation of FPSP. In the following, these ideas are extended to general boundary controlled DCR-systems (2.39)-(2.42) satisfying the conditions of Theorem 2.4. For the sake of simplicity, it is assumed that $M=K$, i.e. the number $M$ of boundary inputs $\boldsymbol{u}(t)$ equals the number $K$ of states $\boldsymbol{x}(z, t)$ of the DCR-system. Further remarks illustrate the approach if $M<K$.

\subsubsection{Finite-dimensional design model via formal power series and summation methods}

In order to motivate the re-interpretation of formal power series parameterizability for nonlinear systems, recall the constituting equations for the differential recursion (2.47) with starting condition (2.48) for the coefficients $\hat{\boldsymbol{x}}_{n}(t), n \in \mathbb{N}_{0}$ of the series ansatz $\hat{\boldsymbol{x}}(z, t)=\sum_{n=0}^{\infty} \hat{\boldsymbol{x}}_{n}(t) z^{n}$, i.e.

$$
\begin{aligned}
& \dot{\hat{\boldsymbol{x}}}_{n}(t)-(n+2)(n+1) S \hat{\boldsymbol{x}}_{n+2}(t)-\hat{\boldsymbol{\Psi}}_{n}\left(\underline{\hat{\boldsymbol{x}}}_{n+1}(t)\right)=\mathbf{0}, \quad \forall n \in \mathbb{N}_{0} \\
& P_{0} \hat{\boldsymbol{x}}_{1}(t)+R_{0} \hat{\boldsymbol{x}}_{0}(t)=\boldsymbol{\zeta}(t) .
\end{aligned}
$$

Thereby it is assumed that the nonlinear source term $\boldsymbol{\Psi}(\boldsymbol{x}, \partial \boldsymbol{x} / \partial z)$ satisfies Assumption 2.1. For the derivation of the state and input parameterization in Chapter 2, Eqn. (5.24) is solved for the state $\hat{\boldsymbol{x}}_{n+2}(t)$ by imposing additional BCs (2.50) involving a parameterizing function. This leads to Theorem 2.4 on the formal power series parameterizability of the DCR-system 
(2.39)-(2.42). On the other hand, Eqn. (5.24) can be equivalently re-written as a set of nonlinear homogeneous equations satisfying

$$
\dot{\hat{\boldsymbol{x}}}_{n}(t)=(n+2)(n+1) S \hat{\boldsymbol{x}}_{n+2}(t)+\hat{\mathbf{\Psi}}_{n}\left(\underline{\hat{\boldsymbol{x}}}_{n+1}(t)\right), \quad \forall n \in \mathbb{N}_{0}
$$

From this set, it suffices to consider only the equations with even index $n$, since due to (5.25) the coefficients $\hat{\boldsymbol{x}}_{2 n+1}$ can be expressed algebraically in terms of the coefficients $\hat{\boldsymbol{x}}_{2 j}, j=0,1, \ldots, n$ with even index as can be easily verified by induction. This is briefly outlined below:

(i) From (5.25) for $M=K$ it follows that $\hat{\boldsymbol{x}}_{1}=P_{0}^{-1}\left(\boldsymbol{\zeta}-R_{0} \hat{\boldsymbol{x}}_{0}\right)=: \boldsymbol{\vartheta}_{1}\left(\hat{\boldsymbol{x}}_{0}, \boldsymbol{\zeta}\right)$.

(ii) Evaluation of (5.24) or respectively (5.26) for $n=1$ provides

$$
\begin{aligned}
\hat{\boldsymbol{x}}_{3} & =\frac{S^{-1}}{6}\left(\dot{\hat{\boldsymbol{x}}}_{1}-\hat{\boldsymbol{\Psi}}_{1}\left(\underline{\hat{\boldsymbol{x}}}_{n+1}\right)\right) \\
& \stackrel{(i)}{=} \frac{S^{-1}}{6}\left(\frac{\partial \boldsymbol{\vartheta}_{1}}{\partial \hat{\boldsymbol{x}}_{0}} \dot{\boldsymbol{x}}_{0}+\frac{\partial \boldsymbol{\vartheta}_{1}}{\partial \boldsymbol{\zeta}} \dot{\boldsymbol{\zeta}}-\hat{\boldsymbol{\Psi}}_{1}\left(\left[\hat{\boldsymbol{x}}_{0}^{T}, \hat{\boldsymbol{x}}_{1}^{T}, \hat{\boldsymbol{x}}_{2}^{T}\right]\right)\right) .
\end{aligned}
$$

Since $\dot{\hat{\boldsymbol{x}}}_{0}=2 S \hat{\boldsymbol{x}}_{2}+\hat{\boldsymbol{\Psi}}_{0}\left(\underline{\hat{\boldsymbol{x}}}_{1}\right)$ from (5.26) and $\hat{\boldsymbol{x}}_{1}=\boldsymbol{\vartheta}_{1}\left(\hat{\boldsymbol{x}}_{0}, \boldsymbol{\zeta}\right)$ from (i), it follows that $\hat{\boldsymbol{x}}_{3}=\boldsymbol{\vartheta}_{3}\left(\hat{\boldsymbol{x}}_{0}, \hat{\boldsymbol{x}}_{2}, \boldsymbol{\zeta}, \dot{\boldsymbol{\zeta}}\right)=: \boldsymbol{\vartheta}_{3}\left(\underline{\hat{\boldsymbol{x}}}_{2}^{\prime}, \underline{\boldsymbol{\zeta}}_{1}\right)$ where

$$
\begin{aligned}
\underline{\hat{\boldsymbol{x}}}_{2 j}^{\prime} & :=\left[\hat{\boldsymbol{x}}_{0}^{T}, \hat{\boldsymbol{x}}_{2}^{T}, \ldots, \hat{\boldsymbol{x}}_{2 j}^{T}\right]^{T} \\
\underline{\boldsymbol{\zeta}}_{j} & :=\left[\boldsymbol{\zeta}^{T}, \dot{\boldsymbol{\zeta}}^{T}, \ldots, \boldsymbol{\zeta}^{(j)^{T}}\right]^{T}
\end{aligned}
$$

for $j \in \mathbb{N}_{0}$.

(iii) Induction yields the result that

$$
\hat{\boldsymbol{x}}_{2 n+1}=\boldsymbol{\vartheta}_{2 n+1}\left(\underline{\hat{\boldsymbol{x}}}_{2 n}^{\prime}, \underline{\boldsymbol{\zeta}}_{n}\right), \quad n \in \mathbb{N}_{0} .
$$

In view of (5.27), the set (5.26) reduces to

$$
\dot{\hat{\boldsymbol{x}}}_{2 n}(t)=(2 n+2)(2 n+1) S \hat{\boldsymbol{x}}_{2 n+2}(t)+\hat{\boldsymbol{\chi}}_{2 n}\left(\underline{\hat{\boldsymbol{x}}}_{2 n}^{\prime}, \underline{\boldsymbol{\zeta}}_{n}\right),
$$

where

$$
\hat{\boldsymbol{\chi}}_{2 n}\left(\underline{\hat{\boldsymbol{x}}}_{2 n}^{\prime}, \underline{\boldsymbol{\zeta}}_{n}\right):=\hat{\boldsymbol{\Psi}}_{2 n}(\underbrace{\left.\hat{\boldsymbol{x}}_{0}^{T}, \boldsymbol{\vartheta}_{1}^{T}\left(\underline{\hat{\boldsymbol{x}}}_{0}^{\prime}, \underline{\boldsymbol{\zeta}}_{0}\right), \hat{\boldsymbol{x}}_{2}^{T}, \boldsymbol{\vartheta}_{3}^{T}\left(\underline{\hat{\boldsymbol{x}}}_{2}^{\prime}, \underline{\boldsymbol{\zeta}}_{1}\right), \ldots, \hat{\boldsymbol{x}}_{2 n}^{T}, \boldsymbol{\vartheta}_{2 n+1}^{T}\left(\underline{\hat{\boldsymbol{x}}}_{2 n}^{\prime}, \underline{\boldsymbol{\zeta}}_{n}\right)\right]^{T}}_{=: \underline{\hat{\boldsymbol{x}}}_{2 n+1}\left(\underline{\hat{\boldsymbol{x}}}_{2 n}^{\prime}, \underline{\boldsymbol{\zeta}}_{n}\right)}) .
$$

In Meurer (2002) and Meurer and Zeitz (2003b) it is shown that an inherently flat finitedimensional controller design model can be derived under the formal assumption of an at least unit radius of convergence of the series $\hat{\boldsymbol{x}}(z, t)$. This formally allows a truncation of the set of equations (5.28) at some integer $n=N-1, N>1$. The main idea is based on the reinterpretation of (5.28) for $n \in\{0,1, \ldots, N-1\}$ as sets of ODEs for the coefficients of the formal power series with even index. As can be directly deduced from the structure of (5.28), any set 
of ODEs is characterized by a forward coupling into the states of the following set of ODEs due to the terms $\hat{\boldsymbol{x}}_{2 n+2}$ such that by truncation at $n=N-1$ the variables $\hat{\boldsymbol{x}}_{2 N}$ remain in the ODEs for $\hat{\boldsymbol{x}}_{2 N-2}$. Recall that in Section 2.2.1, the differential recursions for the formal power series parameterization are expressed in terms of $\hat{\boldsymbol{x}}_{n+2}$, or respectively $\hat{\boldsymbol{x}}_{2 n+2}$, by imposing BCs (2.50) and formally neglecting the controlled BCs (2.42). However, under the formal assumption of convergence, a finite-dimensional design model is obtained by replacing $\hat{\boldsymbol{x}}_{2 N}$ in (5.28) for $n=N-1$ using an appropriate truncation of the formal power series parameterization of the input terms (2.49), i.e.

$$
\hat{\boldsymbol{u}}(t)=Q^{-1} \sum_{n=0}^{\infty}\left[R_{1} \hat{\boldsymbol{x}}_{n}(t)+(n+1) P_{1} \hat{\boldsymbol{x}}_{n+1}(t)\right]
$$

to obtain

$$
\hat{\boldsymbol{u}} \approx Q^{-1} \sum_{n=0}^{2 N-1}\left[R_{1} \hat{\boldsymbol{x}}_{n}+(n+1) P_{1} \hat{\boldsymbol{x}}_{n+1}\right]=: \sum_{n=0}^{2 N-1} \hat{\boldsymbol{u}}_{n}
$$

where $\hat{\boldsymbol{u}}_{n}:=\left[\hat{u}_{1, n}, \ldots, \hat{u}_{K, n}\right]^{T}$. As illustrated in Chapters 3 and 4 , the convergence requirement can be replaced by the less restrictive assumption of $k$-summability. Hence instead of truncating (5.29) at $n=2 N-1$ its $(2 N-1, \xi)$-approximate $k$-sum $\mathcal{S}_{\boldsymbol{k}}^{2 N-1, \boldsymbol{\xi}}$ defined in (3.25) is introduced

$$
\hat{u}_{i} \approx \frac{\sum_{n=0}^{2 N-1}\left(\sum_{j=0}^{n} \hat{u}_{i, j}\right) \frac{\xi_{i}^{n}}{\Gamma\left(1+\frac{n}{k_{i}}\right)}}{\sum_{n=0}^{2 N-1} \frac{\xi_{i}^{n}}{\Gamma\left(1+\frac{n}{k_{i}}\right)}}, \quad i=1,2, \ldots, K
$$

with summation parameters $\boldsymbol{\xi}=\left(\xi_{1}, \ldots, \xi_{K}\right)$ and $\boldsymbol{k}=\left(k_{1}, \ldots, k_{K}\right)$. These expressions for $i=1, \ldots, K$ allow a matrix representation of the form

$$
\hat{\boldsymbol{u}}=V_{\boldsymbol{\xi}, \boldsymbol{k}} \sum_{n=0}^{2 N-1} W_{\boldsymbol{\xi}, \boldsymbol{k}}(n) \sum_{j=0}^{n} \hat{\boldsymbol{u}}_{j}
$$

with matrices

$$
\begin{gathered}
V_{\boldsymbol{\xi}, \boldsymbol{k}}=\operatorname{diag}\left\{1 / \sum_{n=0}^{2 N-1} \frac{\xi_{i}^{n}}{\Gamma\left(1+\frac{n}{k_{i}}\right)}\right\}_{i=1, \ldots, K} \in \mathbb{R}^{K \times K} \\
W_{\boldsymbol{\xi}, \boldsymbol{k}}(n)=\operatorname{diag}\left\{\frac{\xi_{i}^{n}}{\Gamma\left(1+\frac{n}{k_{i}}\right)}\right\}_{i=1, \ldots, K} \in \mathbb{R}^{K \times K} .
\end{gathered}
$$

In order to substitute $\hat{\boldsymbol{x}}_{2 N}$ in (5.28) for $n \in\{0,1, \ldots, N-1\}$, solve (5.30) for this remaining unknown. Assuming that $P_{1} \neq \mathbb{O}_{K}$, if follows after some intermediate computations that

$$
\hat{\boldsymbol{x}}_{2 N}=-\sum_{n=0}^{N-1} \mathcal{A}_{\boldsymbol{\xi}, \boldsymbol{k}}^{N}(2 n) \hat{\boldsymbol{x}}_{2 n}+\mathcal{B}_{\boldsymbol{\xi}, \boldsymbol{k}}^{N} \hat{\boldsymbol{u}}-\sum_{n=0}^{N-1} \mathcal{A}_{\boldsymbol{\xi}, \boldsymbol{k}}^{N}(2 n+1) \boldsymbol{\vartheta}_{2 n+1}\left(\underline{\hat{\boldsymbol{x}}}_{2 n}^{\prime}, \underline{\boldsymbol{\zeta}}_{n}\right) \text {. }
$$


Thereby

$$
\begin{aligned}
\mathcal{A}_{\boldsymbol{\xi}, \boldsymbol{k}}^{N}(n)= & \bar{P}_{1}^{-1}(2 N-1)\left\{W_{\boldsymbol{\xi}, \boldsymbol{k}}^{-1}(2 N-1) W_{\boldsymbol{\xi}, \boldsymbol{k}}(n-1) \bar{P}_{1}(n-1)+\right. \\
& \left.\left(\mathbb{I}_{K}+W_{\boldsymbol{\xi}, \boldsymbol{k}}^{-1}(2 N-1) \sum_{j=n}^{2 N-2} W_{\boldsymbol{\xi}, \boldsymbol{k}}(j)\right)\left[\bar{P}_{1}(n-1)+\bar{R}_{1}\right]\right\}, \quad n=0,1, \ldots, 2 N-1 \\
\mathcal{B}_{\boldsymbol{\xi}, \boldsymbol{k}}^{N}= & \bar{P}_{1}^{-1}(2 N-1) W_{\boldsymbol{\xi}, \boldsymbol{k}}^{-1}(2 N-1) V_{\boldsymbol{\xi}, \boldsymbol{k}}^{-1}
\end{aligned}
$$

with $\bar{P}_{1}(j)=(j+1) Q^{-1} P_{1}$ for $j \in \mathbb{N}_{0}, \bar{R}_{1}=Q^{-1} R_{1}$, and $\mathbb{I}_{K}$ the $K \times K$ unit matrix. Note that a similar result can be obtained when $P_{1}=\mathbb{O}_{K}$ following Remark 5.5. Substitution of (5.31) into (5.28) for $n=N-1$ results in a finite-dimensional approximation of the boundary controlled DCR-system (2.39)-(2.42) by the set of ODEs (5.34), (5.35) depicted in Figure 5.5 with the state $\underline{\hat{\boldsymbol{x}}}_{2 N-2}^{\prime}=\left[\hat{\boldsymbol{x}}_{0}^{T}, \hat{\boldsymbol{x}}_{2}^{T}, \ldots, \hat{\boldsymbol{x}}_{2 N-2}^{T}\right]^{T} \in \mathbb{R}^{K \cdot N}$ and the input vector $\boldsymbol{u} \in \mathbb{R}^{K}$. These equations can be re-written as an input-affine nonlinear multiple-input system in abbreviated form

$$
\begin{aligned}
& {\underline{\dot{\boldsymbol{x}}^{\prime}}}_{2 N-2}^{\prime}=A \underline{\hat{\boldsymbol{x}}}_{2 N-2}^{\prime}+\boldsymbol{f}\left(\underline{\hat{\boldsymbol{x}}}_{2 N-2}^{\prime}, \underline{\boldsymbol{\zeta}}_{N-1}\right)+B \hat{\boldsymbol{u}}, \quad t>0 \\
& \underline{\hat{\boldsymbol{x}}}_{2 N-2}^{\prime}(0)=\underline{\hat{\boldsymbol{x}}}_{2 N-2,0}^{\prime}
\end{aligned}
$$

with $A, B \in \mathbb{R}^{K \cdot N \times K \cdot N}$ since $\hat{\boldsymbol{u}} \in \mathbb{R}^{K}$, and $\boldsymbol{f} \in \mathbb{R}^{K \cdot N}$. Supplemented by the imposed BCs (2.50) for the parameterizing function $\boldsymbol{y}$ as introduced in Section 2.2.1, i.e.

$$
\begin{aligned}
& \boldsymbol{y}=\left(F_{v}-F_{d} P_{0}^{-1} R_{0}\right) \hat{\boldsymbol{x}}_{0}+F_{d} P_{0}^{-1} \boldsymbol{\zeta} \\
& =\left(\begin{array}{llll}
F_{v}-F_{d} P_{0}^{-1} R_{0} & O_{K} & \ldots & O_{K}
\end{array}\right) \underline{\hat{x}}_{2 N-2}^{\prime}+\left(\begin{array}{llll}
F_{d} P_{0}^{-1} & O_{K} & \ldots & \mathbb{O}_{K}
\end{array}\right) \underline{\boldsymbol{\zeta}}_{N-1} \\
& =C{\underline{\hat{\boldsymbol{x}}_{2 N-2}^{\prime}}}_{2+E \underline{\boldsymbol{\zeta}}_{N-1}},
\end{aligned}
$$

and provided that the condition of Theorem 2.4 on FPSP is satisfied, the control system (5.36)(5.38) allows an interpretation as a generalized controller normal form. This is due to the parabolic character of the DCR-system (2.39) such that $S \in K \times K$ can be assumed diagonal. Hence as can be seen from (5.34), the system matrix $A$ exhibits a band-structure with non-zero elements along the upper diagonal $A_{n, K+n}, n=1, \ldots,(N-1) K$ and along the last $K$ rows. In addition, the vector $\boldsymbol{f}\left(\underline{\hat{\boldsymbol{x}}}_{2 N-2}^{\prime}, \underline{\boldsymbol{\zeta}}_{N-1}\right)$ with known time-variable coefficients $\underline{\boldsymbol{\zeta}}_{N-1}$, is in triangular form with respect to $\underline{\hat{x}}_{2 N-2}^{\prime}$.

In Section 5.2.2, it is illustrated for a simplified linear version of the considered tubular reactor example of Section 2.2.2, that the derived finite-dimensional model (5.36)-(5.37) indeed represents a sufficient and accurate approximation to the given DPS. This fact is also exploited in the following section for the design of flatness-based tracking control with observer.

\subsubsection{Flatness-based tracking control with observer}

As a direct consequence of the structure of the ODEs (5.36), it can be verified that $\boldsymbol{y}(t)$ defined by (5.38) has a relative degree $\boldsymbol{r}=\sum_{j=1}^{K} r_{j}=K \cdot N$, whereby $r_{j}=N$ for any $j$. Hence 


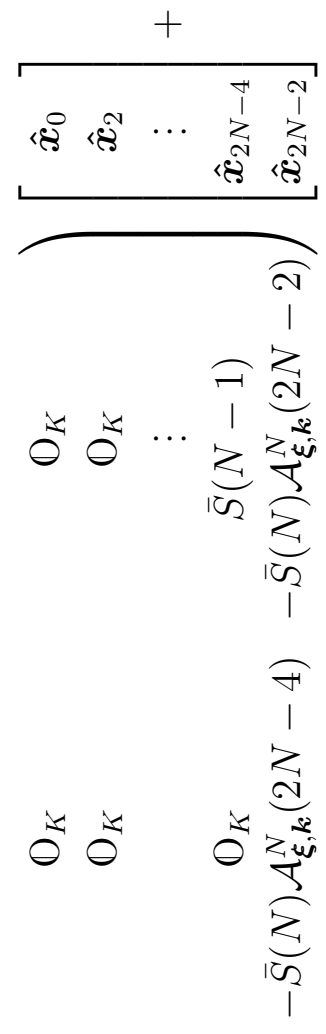

落

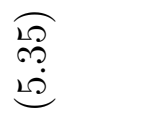

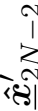

总蓠

용

爱

간

ํ.

के

ণิ

द्व

范

穵

藏

च્0

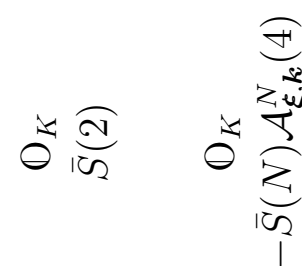

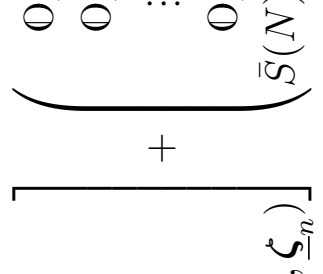

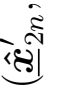

$\vdots \quad \therefore \quad \vdots \quad \vdots$
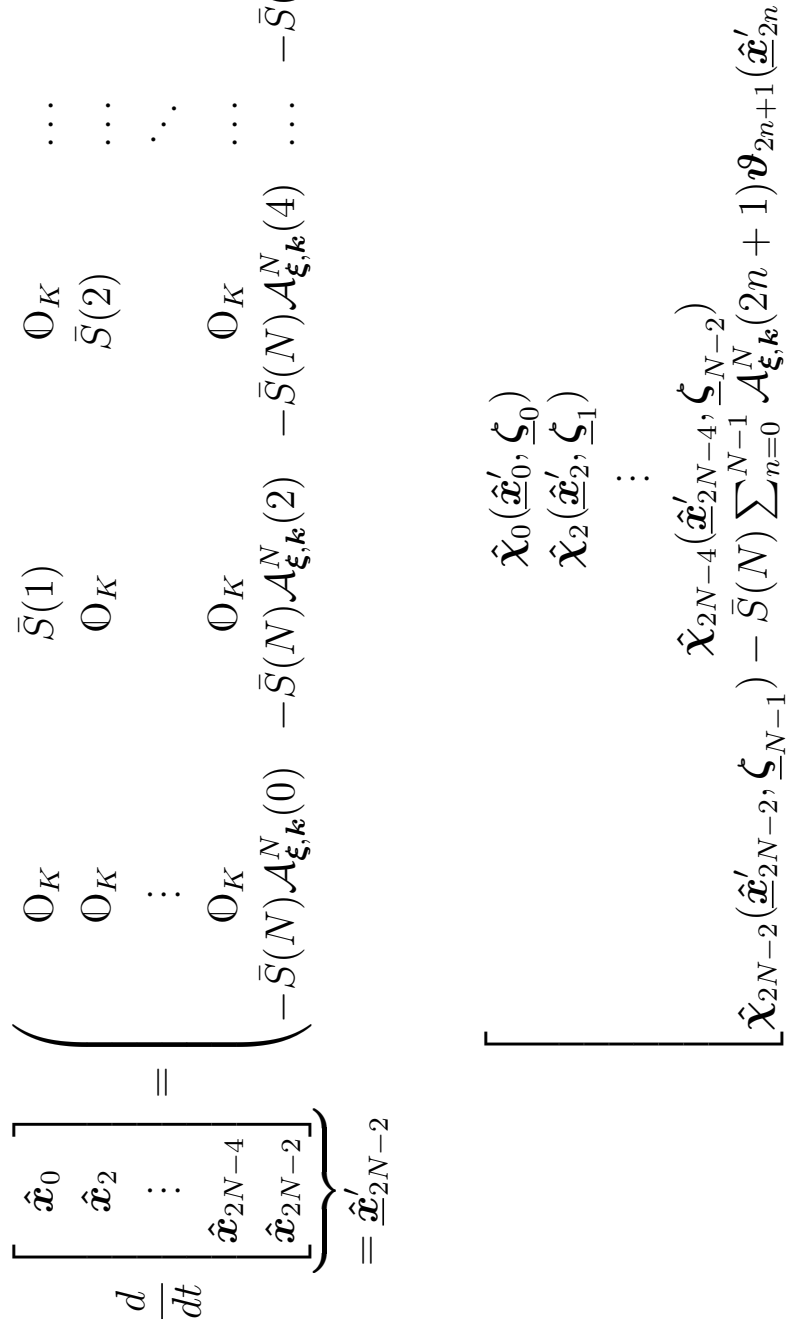

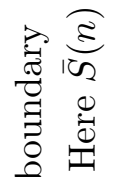

胥运

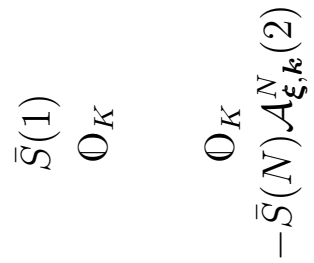

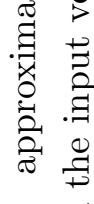

శ్రేี

.

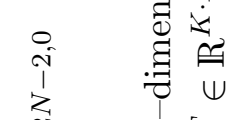

«स्ते|

莺

อ 社

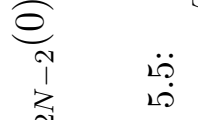

रहै|

कूष सं०

.006 
the system (5.36) is differentially flat with flat output (5.38), such that the states $\underline{\hat{\boldsymbol{x}}}_{2 n}^{\prime}(t)$, $n=0,1, \ldots, N-1$ and inputs $\boldsymbol{u}(t)$ can be expressed in terms of $\boldsymbol{y}(t)^{3}$ and its time-derivatives (Fliess et al., 1995). In the sequel, this property is exploited for the design of asymptotically stabilizing feedback tracking control for the DCR-system (2.39)-(2.42).

Since flat systems are exactly feedback linearizable, asymptotic tracking control can be designed by means of linear control theory - for details the reader is referred to Fliess et al. (1995); Rothfuß (1997); Martin et al. (1997). Following this approach for (5.36)-(5.38), a static feedback law is obtained

$$
\hat{\boldsymbol{u}}=\boldsymbol{\Theta}\left(\boldsymbol{y}, \dot{\boldsymbol{y}}, \ldots, \boldsymbol{y}^{(N-1)}, \boldsymbol{y}_{d}^{(N)}(t)-\boldsymbol{\Lambda}(\underline{\boldsymbol{e}})\right)
$$

where $\boldsymbol{\Lambda}(\underline{\boldsymbol{e}})=\left[\Lambda_{1}^{T}\left(\boldsymbol{e}_{1}\right), \Lambda_{2}^{T}\left(\boldsymbol{e}_{2}\right), \ldots, \Lambda_{K}^{T}\left(\boldsymbol{e}_{K}\right)\right]^{T}$ with $\boldsymbol{e}_{i}:=\left[e_{i}^{T}, \dot{e}_{i}^{T}, \ldots, e_{i}^{(N-1)^{T}}\right]^{T}$ can be any type of feedback control asymptotically stabilizing the tracking error $e_{i}=y_{i}-y_{i, d}, i=1,2, \ldots, K$. In the sequel, $\mathrm{PID}_{N}$ control is considered for the design of $\boldsymbol{\Lambda}(\underline{\boldsymbol{e}})$, i.e.

$$
\Lambda_{i}\left(\boldsymbol{e}_{i}\right)=p_{i, 0} \int_{0}^{t} e_{i}(\tau) d \tau+\sum_{j=1}^{N} p_{i, j} e_{i}^{(j)}
$$

where the parameters $p_{i, j}$ for $i=1,2, \ldots, K, j=0,1, \ldots, N$ are assumed to be coefficients of Hurwitz polynomials and can hence be determined by eigenvalue assignment and comparison of coefficients

$$
\varrho^{N+1}+p_{i, N} \varrho^{N}+\ldots+p_{i, 1} \varrho+p_{i, 0}=\prod_{j=0}^{N}\left(\varrho-\varrho_{i, j}\right) \quad \text { for } i=1, \ldots, K .
$$

Obviously implementation of the derived feedback law (5.39) requires knowledge of the full state information. Hence an observer is required to estimate the non-measured states. As shown in Chapter 2 for linear and nonlinear examples of DCR-systems, the from a theoretical point of view fictious parameterizing function $\boldsymbol{y}$ typically exhibits a physically meaningful behavior. Therefore, it is assumed in the following that $\boldsymbol{y}$ from (5.38) is the measured quantity, such that the verification of flatness by sequential time-differentiation of $\boldsymbol{y}$ under consideration of the system ODEs (5.36) directly corresponds to the proof of observability. Following the work of Rothfuß et al. (1996) and Rothfuß (1997), a nonlinear tracking observer can be designed based on the finite-dimensional model (5.36)-(5.38), i.e.

$$
\begin{aligned}
& \frac{d \underline{\hat{\boldsymbol{x}}}_{2 N-2}^{\prime}}{d t}=A{\underline{\tilde{\boldsymbol{x}}^{\prime}}}_{2 N-2}^{\prime}+\boldsymbol{f}\left({\underline{\tilde{\boldsymbol{x}}^{\prime}}}_{2 N-2}^{\prime}, \underline{\boldsymbol{\zeta}}_{N-1}\right)+B \tilde{\hat{\boldsymbol{u}}}+L(t)\left[\boldsymbol{y}-C{\underline{\tilde{\boldsymbol{x}}_{2 N-2}^{\prime}}}_{2}^{\prime} E \underline{\boldsymbol{\zeta}}_{N-1}\right], \\
& \underline{\hat{\boldsymbol{x}}}_{2 N-2}^{\prime}(0)=\underline{\hat{\boldsymbol{x}}}_{2 N-2,0}^{\prime}
\end{aligned}
$$

with reasonable ICs $\underline{\hat{\boldsymbol{x}}}_{2 N-2,0}^{\prime}$. The time-variable observer gain matrix $L(t) \in \mathbb{R}^{+} \times \mathbb{R}^{K \cdot N \times K}$ can be determined based on a linearization of the observer error dynamics along the desired states

\footnotetext{
${ }^{3}$ Recall that $\boldsymbol{y}(t)=F_{v} \boldsymbol{x}(0, t)+F_{d} \partial \boldsymbol{x} / \partial z(0, t)$, which is reflected in (5.38).
} 
$\underline{\hat{\boldsymbol{x}}}_{2 N-2, d}^{\prime}(t)$ which are known due to the flatness property of (5.36) (Rothfuß et al., 1996). This allows the application of the MIMO time-variable Ackermann formula for the design of $L(t)$ by means of pole placement (Freund, 1971). Thus, appropriate eigenvalues have to be assigned for the observer error dynamics

$$
\tilde{\varrho}^{N}+q_{i, N} \tilde{\varrho}^{N}+\ldots+q_{i, 1} \tilde{\varrho}+q_{i, 0}=\prod_{j=0}^{N-1}\left(\varrho \tilde{\varrho}-\tilde{\varrho}_{i, j}\right) \quad \text { for } i=1, \ldots, K .
$$

The following remark briefly concerns observer design if the measured quantities and the parameterizing function differ:

Remark 5.6. Assume for the sake of simplicity that there exists a linear operator $\mathcal{M}$ relating the state $\boldsymbol{x}(z, t)$ of the DCR-system and the vector of measurements $\boldsymbol{\eta}(t)$, i.e.

$$
\boldsymbol{\eta}(t)=\mathcal{M} \boldsymbol{x}(z, t), \quad z \in[0,1]
$$

If $\boldsymbol{x}(z, t)$ can be sufficiently approximated by the truncated series $\hat{\boldsymbol{x}}_{N}(z, t)=\sum_{n=0}^{2 N-1} \hat{\boldsymbol{x}}_{n}(t) z^{n}$ it follows in view of (5.27), that

$$
\boldsymbol{\eta}=\mathcal{M} \hat{\boldsymbol{x}}_{N}(z, t)=\mathcal{M} \sum_{n=0}^{N-1} \hat{\boldsymbol{x}}_{2 n}(t) z^{2 n}+\mathcal{M} \sum_{n=0}^{N-1} \boldsymbol{\vartheta}_{2 n+1}\left(\underline{\hat{\boldsymbol{x}}}_{2 n}^{\prime}, \underline{\boldsymbol{\zeta}}_{n}\right) z^{2 n+1}=: \boldsymbol{\Xi}\left(\underline{\hat{\boldsymbol{x}}}_{2 N-2}^{\prime}\right) .
$$

Note that alternatively, the $(2 N-1, \xi)$-approximate $k$-sum can be applied, i.e. $\boldsymbol{x}(z, t) \approx$ $\mathcal{S}_{\boldsymbol{k}}^{2 N-1, \boldsymbol{\xi}}(\hat{\boldsymbol{x}}(z, t))$ to improve the quality of the approximation. In either case, observer design is possible, if the design model (5.36) is observable with respect to $\boldsymbol{\eta}=\boldsymbol{\Xi}\left(\underline{\hat{\boldsymbol{x}}}_{2 N-2}^{\prime}\right)$. If this is ensured, similar to the design above, a time-variant observer gain $L(t)$ can be determined based on the linearization of (5.36), (5.45) along the desired states $\underline{\hat{\boldsymbol{x}}}_{2 N-2, d}^{\prime}$, which are known due to the flatness property of the design model (Rothfuß, 1997).

A similar procedure is possible for nonlinear operators $\mathcal{M}$ under the assumption that $\mathcal{M} \hat{\boldsymbol{x}}(z, t)$ can be approximated by a nonlinear function in terms of $\underline{\hat{x}}_{2 N-2}^{\prime}$.

Since the designed observer (5.42)-(5.44) provides an estimate $\underline{\hat{\boldsymbol{x}}}_{2 N-2}^{\prime}$ of the series coefficients with even index summarized in the state vector $\underline{\hat{\boldsymbol{x}}}_{2 N-2}^{\prime}$, an approximation of the evolution of the spatial profile is simultaneously obtained.

\subsubsection{Spatial profile estimation}

In addition to the realization of state feedback, the designed observer can be used for spatial profile estimation throughout the transition process (Meurer and Zeitz, 2003b; Meurer et al., $2003)$. An estimate $\tilde{\boldsymbol{x}}(z, t)$ of the spatial profile $\boldsymbol{x}(z, t)$ is obtained by an evaluation of the first $2 N+1$ terms of formal power series ansatz $\hat{\boldsymbol{x}}(z, t)=\sum_{n=0}^{\infty} \hat{\boldsymbol{x}}_{n}(t) z^{n}$ with the coefficients $\hat{\boldsymbol{x}}_{n}(t)$ being replaced by their estimated counterparts $\tilde{\hat{\boldsymbol{x}}}_{n}(t)$. Thereby, the algebraic relations (5.27) 
between the coefficients with odd and even index as well as Eqn. (5.31) for $\hat{\boldsymbol{x}}_{2 N}$ with the known input $\tilde{\hat{\boldsymbol{u}}}$ are utilized, i.e.

$$
\tilde{\hat{\boldsymbol{x}}}(z, t)=\sum_{n=0}^{2 N} \tilde{\hat{\boldsymbol{x}}}_{n}^{\prime}(t) z^{n}=\sum_{n=0}^{N-1} \tilde{\hat{\boldsymbol{x}}}_{2 n}^{\prime}(t) z^{2 n}+\tilde{\hat{\boldsymbol{x}}}_{2 N}^{\prime}\left(\underline{\hat{\boldsymbol{x}}}_{2 N-2}^{\prime}, \underline{\boldsymbol{\zeta}}, \tilde{\hat{\boldsymbol{u}}}\right) z^{2 N}+\sum_{n=0}^{N-1} \boldsymbol{\vartheta}_{2 n+1}\left(\tilde{\hat{\boldsymbol{x}}}_{2 n}^{\prime}, \underline{\boldsymbol{\zeta}}^{2 N} z^{2 n+1} .\right.
$$

On the other hand, motivated by the results of Chapter 4 for the application of the $(N, \xi)-$ approximate $k$-sum to feedforward control of boundary controlled DCR-systems, application of summation techniques enables to further improve the results of profile estimation. Hence, the system state $\boldsymbol{x}(z, t)$ can be reconstructed using

$$
\tilde{\hat{\boldsymbol{x}}}(z, t)=V_{\boldsymbol{\xi}, \boldsymbol{k}} \sum_{n=0}^{2 N} W_{\boldsymbol{\xi}, \boldsymbol{k}}(n) \sum_{j=0}^{n} \tilde{\hat{\boldsymbol{x}}}_{j}^{\prime}(t) z^{j}
$$

Summarizing the results of this section, the block diagram depicted in Figure 5.6 is obtained for the realization of the proposed flatness-based feedback control with observer for boundary tracking control of nonlinear DCR-systems. Note that all non-measured states are replaced by their estimated counterpart as indicated by the tilde on each affected quantity. The control loop is extended by the profile estimation (5.46), which online provides additional insight in the process behavior, e.g. for monitoring purposes, without further measurement or design effort.

In the following, the proposed flatness-based approach to tracking control with observer and spatial profile estimation is applied for the boundary control of the non-isothermal tubular reactor model of Section 2.2.2.

\subsubsection{The case $\mathrm{M}<\mathrm{K}$}

If in case of a formal power series parameterizable DCR-system (2.39)-(2.42), the number $K$ of states exceeds the number $M$ of inputs, the determination of a finite-dimensional control and observer design model is more challenging and depends individually on the system structure. In general, the design model has to be formulated in terms of the coefficients of the formal power series related to the controlled PDE subsystem, i.e. the $M$ explicitly boundary controlled PDEs of the overall system. Once the respective differential equations for these coefficients are identified, the introduced approach carries over to this particular case.

\subsubsection{Feedback control for the tubular reactor model}

Based on the results of the previous section on the extension of flatness-based methods to boundary controlled nonlinear DCR-systems, tracking control is designed for the tubular reactor model (2.61)-(2.64) with boundary control. Therefore, the design steps proposed in Section 5.2.1 are applied to the model equations in order to obtain robust tracking control in the presence of model errors. The control performance is illustrated in simulation scenarios. 


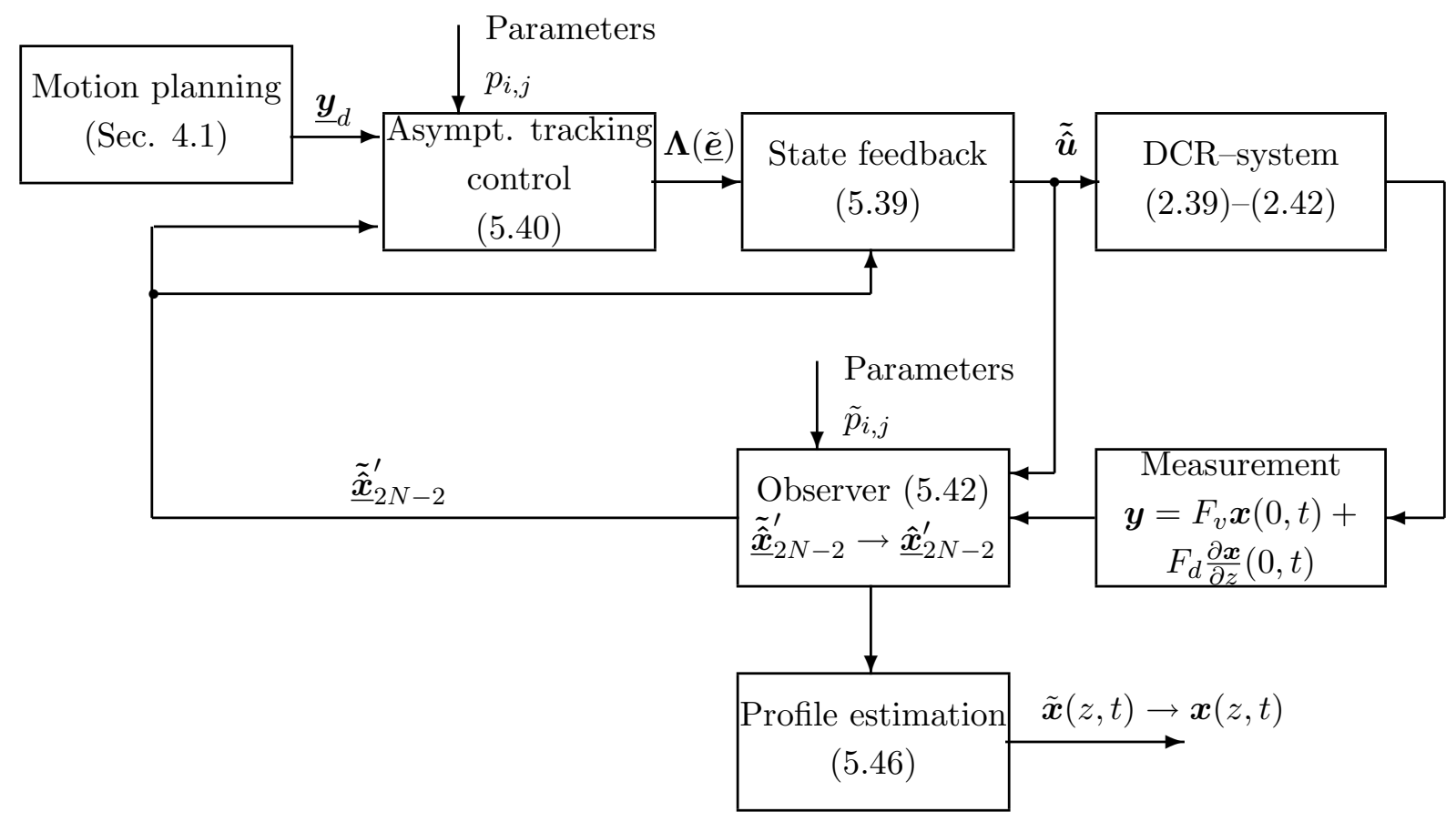

Figure 5.6: Block diagram of the flatness-based boundary tracking control scheme with spatial profile estimation for DPS (2.39)-(2.42) with $\underline{\boldsymbol{y}}_{d}=\left[\boldsymbol{y}_{d}^{T}, \ldots, \boldsymbol{y}_{d}^{(N), T}\right]^{T}$. Note that instead of measuring the parameterizing function $\boldsymbol{y}$, due to Remark 5.6 differing output variables preserving observability of the design model (5.36), (5.37) can be considered equivalently.

\subsubsection{Nonlinear controller normal form}

The control and observer design model for the tubular reactor example can be directly determined from Eqns. (5.32)-(5.35) for the general setup. Therefore, the system, boundary, and input matrices $S, R_{0,1}, P_{0,1}$, and $Q$, respectively, have to be identified. As summarized in Section 2.2.2, these read as

$$
S=\left(\begin{array}{cc}
\frac{L e}{P e_{1}} & 0 \\
0 & \frac{1}{P e_{2}}
\end{array}\right), \quad P_{1}=\left(\begin{array}{cc}
\frac{1}{P e_{1}} & 0 \\
0 & \frac{1}{P e_{2}}
\end{array}\right)
$$

while $R_{0}=\mathrm{O}_{2}$ and $P_{0}=R_{1}=Q=\mathbb{I}_{2}$. Hence Eqns. (5.32), (5.33) reduce to

$$
\begin{aligned}
\mathcal{A}_{\boldsymbol{\xi}, \boldsymbol{k}}^{N}(n) & =\operatorname{diag}\left\{\frac{P e_{i}}{2 N-1}\left[\frac{n}{P e_{i}} \sigma_{i, n-1}+\left(1+\frac{n}{P e_{i}}\right)\left(1+\sum_{j=n}^{2 N-2} \sigma_{i, j}\right)\right]\right\}_{i=1,2} \\
\mathcal{B}_{\boldsymbol{\xi}, \boldsymbol{k}}^{N} & =\left(\begin{array}{cc}
\sum_{n=0}^{2 N-1} \sigma_{1, n} & 0 \\
0 & \sum_{n=0}^{2 N-1} \sigma_{2, n}
\end{array}\right)
\end{aligned}
$$

where $\sigma_{i, j}:=\Gamma\left(1+(2 N-1) / k_{i}\right) / \Gamma\left(1+n / k_{i}\right) \xi^{n-2 N-1}, \sigma_{i,-1}=0$ for $n=0,1, \ldots, 2 N-1$ and $i=1,2$ - see also Meurer and Zeitz (2005). Substitution of (5.47), (5.48) into the 
set of differential equations (5.34) yields the inherently flat, finite-dimensional approximation depicted in Figure 5.7 for the infinite-dimensional tubular reactor model (2.61)-(2.64). Thereby the algebraic dependency (5.27) of the coefficients with odd index on those with even index is verified in Lemma D.1 of the Appendix D.1.

Before studying control and observer design based on (5.49), (5.50), it is shown that these ODEs indeed represent a sufficiently accurate approximation of the system dynamics, if the summation parameters $\boldsymbol{\xi}=\left(\xi_{1}, \xi_{2}\right)$ and $\boldsymbol{k}=\left(k_{1}, k_{2}\right)$ are chosen appropriately for given $N$. This is demonstrated in Figures 5.8-5.10. There the eigenvalue spectrum of a simplified linear version of the tubular reactor model (2.61) given by

$$
\frac{\partial \boldsymbol{x}}{\partial t}=S^{*} \frac{\partial^{2} \boldsymbol{x}}{\partial z^{2}}+\boldsymbol{\Psi}^{*}(\boldsymbol{x})
$$

with

$$
S^{*}=\operatorname{diag}\left\{\frac{1}{P e_{i}}\right\}_{i=1,2}, \quad \Psi^{*}(\boldsymbol{x})=\left[\begin{array}{c}
-\left(x_{1}+x_{2}\right) \\
2\left(x_{1}+x_{2}\right)
\end{array}\right]
$$

and boundary conditions $(2.62),(2.63)$ as above, is depicted and compared to the corresponding spectrum of the system matrix $A$ of the design model (5.49) for different summation parameters $\boldsymbol{\xi}$ and $\boldsymbol{k}$ with $N=10, P e_{1}=P e_{2}=1$. In any case, the dominating and grouped eigenvalues of the operator

$$
L \boldsymbol{x}:=S^{*} \boldsymbol{x}+\Psi^{*}(\boldsymbol{x}),
$$

which is unstable for the chosen parameters, can be approximated with high accuracy for suitable choices of $\boldsymbol{\xi}$ and $\boldsymbol{k}$ in the $(2 N-1, \xi)$-approximate $k$-summation. Furthermore for $\boldsymbol{k}$ decreasing from a value of 1 (Figure 5.8) to a value of $\frac{1}{3}$ (Figure 5.10), also some of the higher modes can be reconstructed in the design model. Note that each subfigure within Figures 5.8-5.10 represents a zoom denoted by $Z 1$ to $Z 4$ into the marked parts of complex eigenvalue domain. Since inverse systems are in general ill-posed (Isakov, 1998), degenerate eigenvalues appear in the spectrum of the design model, which tend into the open right-half complex $s^{-}$ plane along with increasing $\boldsymbol{\xi}$ for given $\boldsymbol{k}$. Nevertheless, from these results it can be deduced that there exist parameters $\boldsymbol{\xi}$ and $\boldsymbol{k}$ for given $N$, such that a suitable approximation of the governing DPS is obtained.

Remark 5.7. The numerical analysis carried out in Figures 5.8-5.10 demonstrates that the introduced re-interpretation allows to extract the fundamental dynamics from a given DPS. Hence for suitable choices of $\boldsymbol{\xi}, \boldsymbol{k}$ (and $N$ ), the proposed approximation approach can be interpreted as a projection onto a low dimensional manifold capturing the essential dynamics in the sense of e.g. Balas (1983); Temam (1988); Sano and Kunimatsu (1995); Christofides (2001) using Galerkin's method and/or (approximate) inertial manifolds.

Similar to Section 5.2.1.2, classical flatness-based methods can be applied for asymptotic tracking control design based on the finite-dimensional approximation (5.49) with flat output (5.50), 


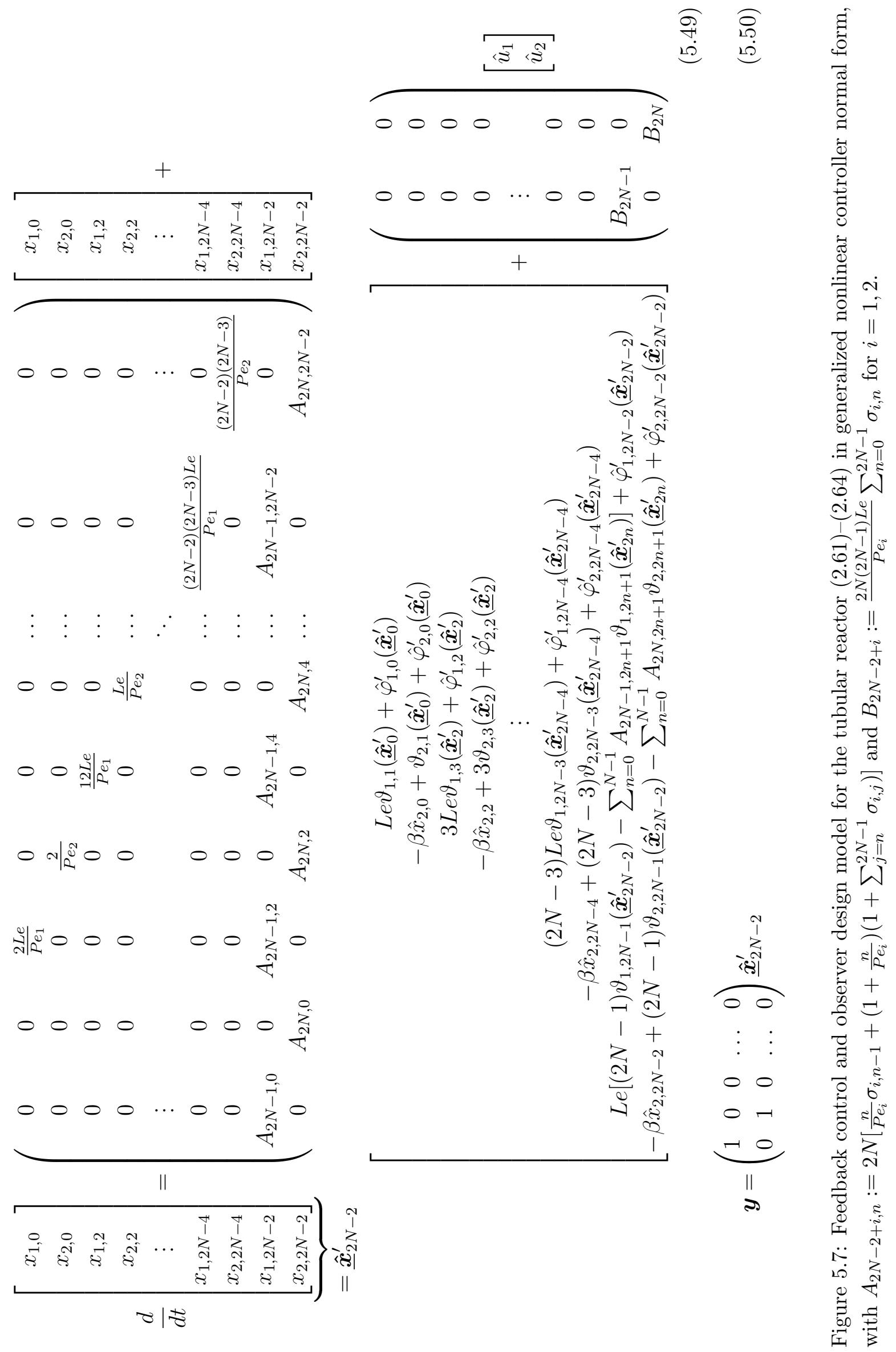



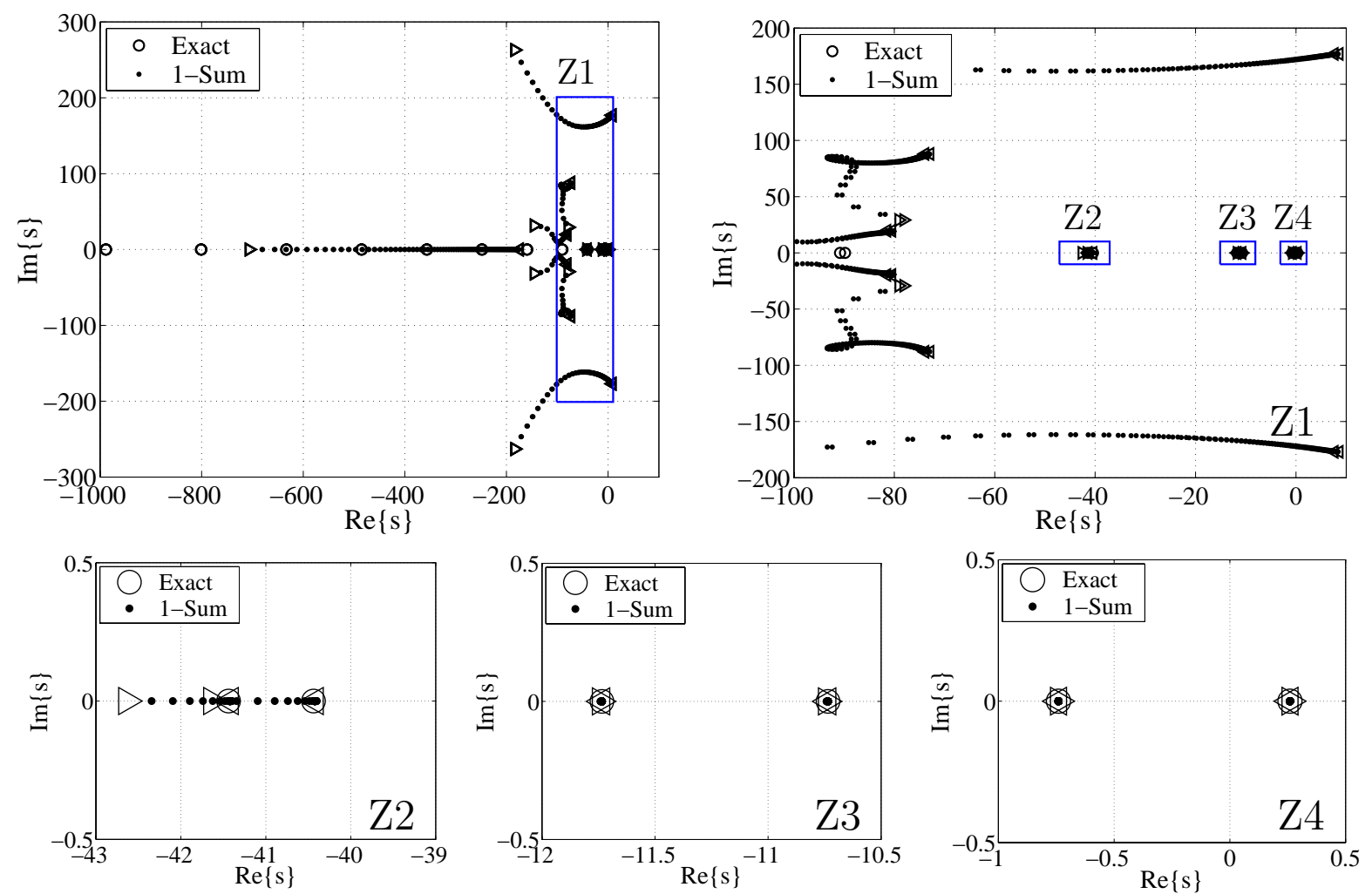

Figure 5.8: Eigenvalue distribution in the complex $s$-domain using $\mathcal{S}_{k}^{2 N-1, \xi}$ with $\xi=\xi_{1}=\xi_{2} \in[13,55]$, $k=k_{1}=k_{2}=1, N=10$ for the linear tubular reactor example (5.51). Here $\circ$ denotes the spectrum of the operator (5.52), $\triangleright$ and $\triangleleft$ correspond to the eigenvalues for $\xi=13$ and $\xi=55$.

which is assumed to represent the controlled variable. This results in the block diagram depicted in Figure 5.6 consisting of state feedback, asymptotic tracking control, observer, spatial profile estimation, and motion planning, which is discussed in Section 4.4.2. Note that any design step can be carried out fully automated using computer-algebra systems such as MATHEMATICA. In the sequel, the flatness-based control scheme is applied to a MOL discretized high-order model of the tubular reactor.

\subsubsection{Simulation results for flatness-based tracking control with observer}

For numerical simulation, the controlled tubular reactor model (2.61)-(2.68) is simulated 'inthe-loop', i.e. a sampling time $\Delta t_{\text {sample }}=0.005$ (dimensionless) is explicitly taken into account. Hence, the controls are assumed constant for each simulation time period $t_{\mathrm{sim}^{i+1}}-t_{\mathrm{sim}^{i}}=\Delta t_{\text {sample }}$ and measurements for the evaluation of the observer are only available at discrete instances of time $t_{\text {sim }^{i+1}}$. This approach directly reflects a possible implementation and allows to determine effects due to the discrete and time-delayed application of the controls.

In order to study the robustness of the proposed flatness-based feedback tracking control scheme (5.39)-(5.41) with observer (5.42)-(5.44) and spatial profile estimation (5.46) based on the design model (5.49), (5.50) when applied to a high-order MOL discretization of the tubular 

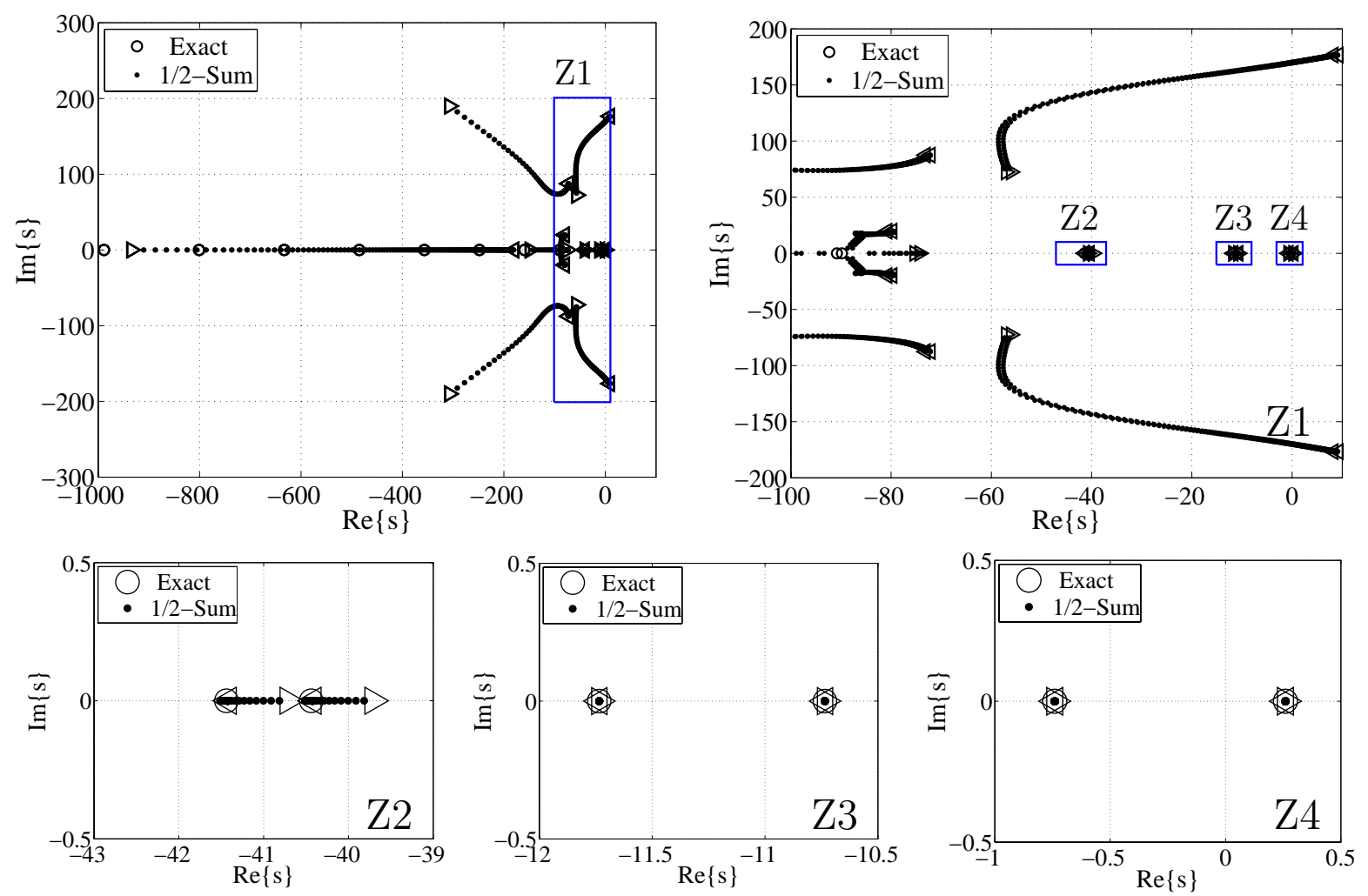

Figure 5.9: Eigenvalue distribution in the complex $s$-domain using $\mathcal{S}_{k}^{2 N-1, \xi}$ with $\xi=\xi_{1}=\xi_{2} \in$ [800,4000], $k=k_{1}=k_{2}=1 / 2, N=10$ for the linear tubular reactor example (5.51). Here $\circ$ denotes the spectrum of the operator (5.52), $\triangleright$ and $\triangleleft$ correspond to the eigenvalues for $\xi=800$ and $\xi=4000$.

reactor, a scenario involving a model error in the $D a$-number is illustrated in Figure 5.11. The $D a-$ number for the simulation model is assumed to differ by $+10 \%$ from the $D a$-number used for the derivation of the feedback control. This intensifies the exothermic reaction due to the influence of $D a$ on both PDEs (2.61). Nevertheless, the desired trajectory is tracked highly accurately by the flatness-based control scheme. For comparison purposes, simulation results for the feedforward controlled tubular reactor model are depicted additionally. Thereby the design model (5.49), (5.50) used for controller synthesis is of dimension 10, i.e. $N=5$. The eigenvalues for the feedback control (5.41) and observer (5.44) are assigned as follows

$$
\begin{aligned}
& \varrho_{i, j}=-4-\frac{j}{2}, \quad j=0,1, \ldots, N \\
& \tilde{\varrho}_{i, j}=-16-j, \quad j=0,1, \ldots, N-1
\end{aligned}
$$

with $i=1,2$. The shown results for profile estimation (Figure 5.11, bottom) clearly prove the applicability of the control concept for process monitoring.

The proposed flatness-based control scheme with state feedback and observer provides robust trajectory tracking in particular for low and medium $P e$-numbers $(\leq 10)$, whereby high $P e$-numbers restrict its applicability. Note that this is a quite common drawback of existing feedback control schemes for DCR-systems as is pointed out by Panfilov and Sheintuch (2001) 

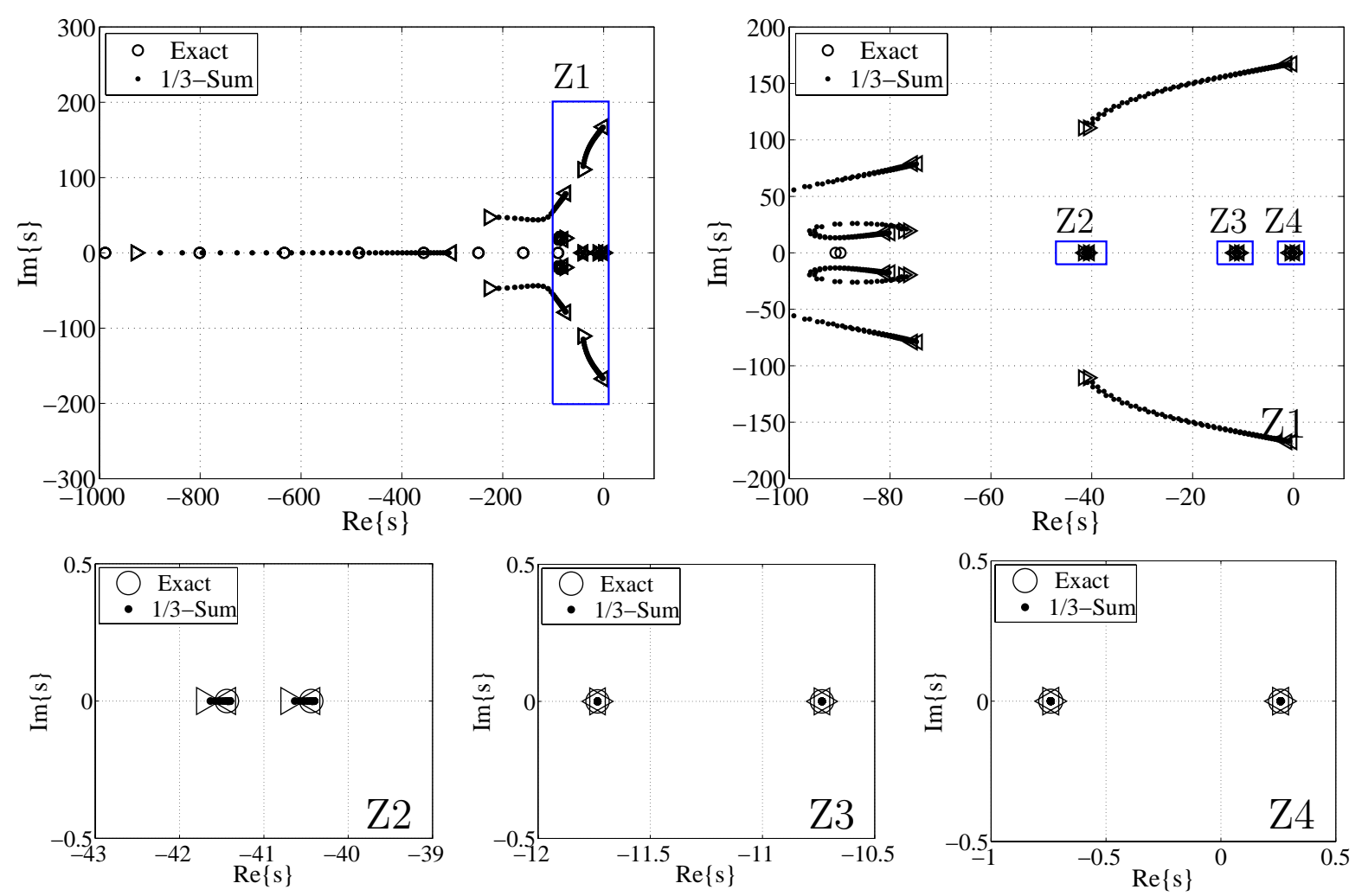

Figure 5.10: Eigenvalue distribution in the complex $s$-domain using $\mathcal{S}_{k}^{2 N-1, \xi}$ with $\xi=\xi_{1}=\xi_{2} \in$ [110000,300000], $k=k_{1}=k_{2}=1 / 3, N=10$ for the linear tubular reactor example (5.51). Here $\circ$ denotes the spectrum of the operator (5.52), $\triangleright$ and $\triangleleft$ correspond to the eigenvalues for $\xi=110000$ and $\xi=300000$.

regarding the work of Christofides (2001); Antoniades and Christofides (2001). On the other hand as illustrated in Section 4.4.2, using the determined feedforward tracking control in conjunction with appropriate summation techniques, provides very accurate trajectory tracking in the nominal case also under severe conditions characterized by high $P e$ and Le numbers. Hence as an alternative to state feedback, application of the two-degree-of-freedom (2DOF) approach (Horowitz, 1963) seems appealing, which supplementing the previously determined feedforward control for the realization of the desired tracking behavior by stabilizing output feedback control (Meurer and Zeitz, 2005). This is briefly outlined in the following section to demonstrate the broad applicability and flexibility of the control design based on FPSP.

\subsubsection{Simulation results for feedforward control and output feedback}

Although accurate feedforward control results can be obtained for the tubular reactor using the proposed $(N, \xi)$-approximate $k$-sum, stabilizing feedback is needed to account for model errors and exogenous disturbances. Within the framework of FPSP, it is possible to project the infinite-dimensional DPS onto a low dimensional manifold reflecting the essential dynamics as shown in the previous section. Starting from the resulting inherently flat approximation, classical flatness-based feedback tracking control design can be performed. 

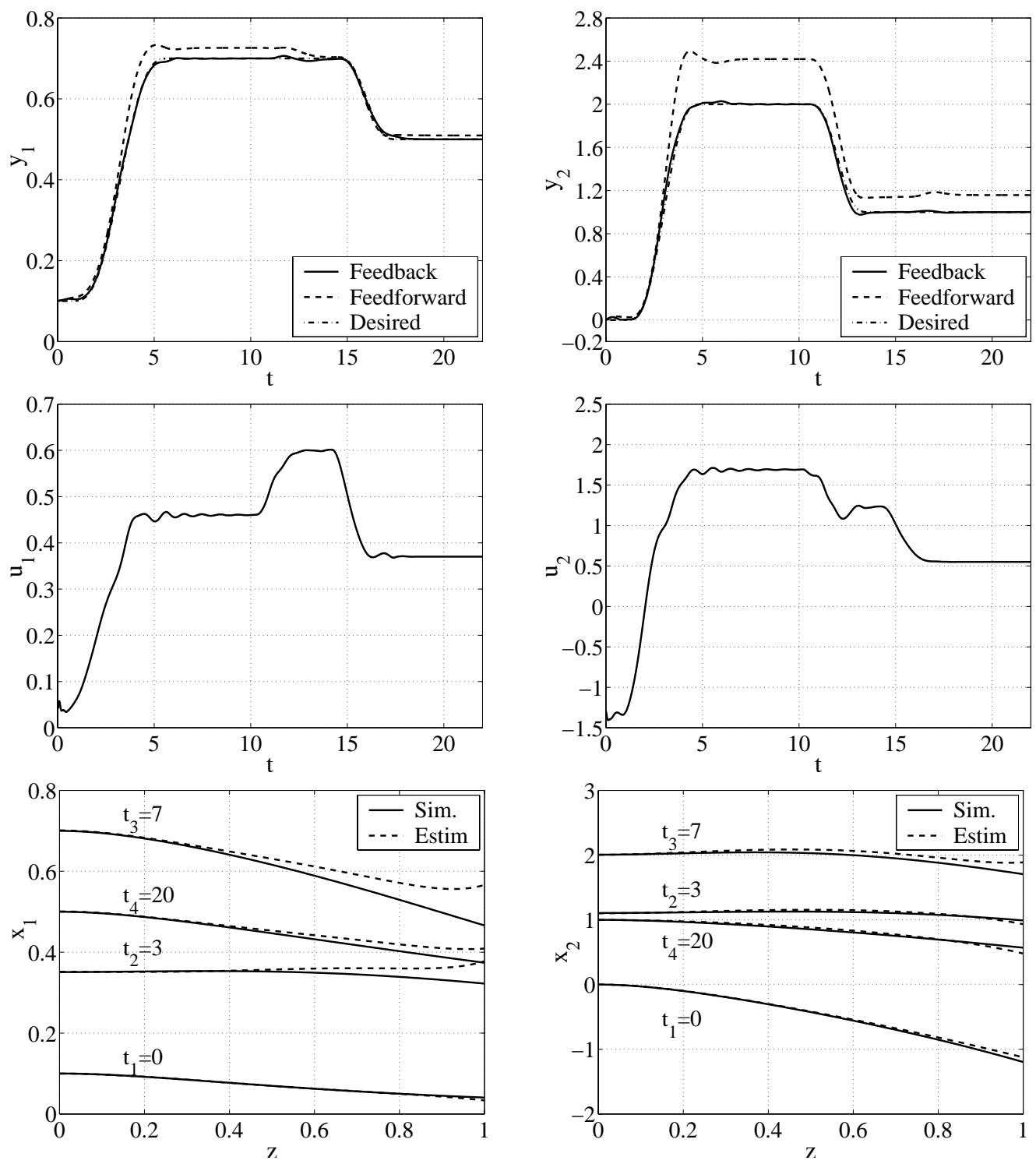

Figure 5.11: Simulation results for flatness-based feedback tracking control with observer (5.39)(5.44) of the tubular reactor model (2.61)-(2.68) with $P e_{1}=P e_{2}=6, L e=1$ and model error, i.e. $D a_{\text {plant }}=1.1 D a_{\text {design }}$. Top: tracking behavior for $x_{1}(0, t)$ and $x_{2}(0, t)$; middle: boundary inputs (5.39); bottom: comparison of estimated profiles $\tilde{x}_{i}\left(z, t_{j}\right), i=1,2$ and simulated profiles $x_{i}\left(z, t_{j}\right), i=1,2$ at different instances of time $t_{j}, j=1,2,3,4$. Remaining parameters are summarized in Table D.1.

On the other hand, the desired profile $\boldsymbol{x}_{d}(z, t)$ is known with sufficient accuracy due to the property of FPSP and the emerging state parameterization (2.76) in terms of the parameterizing function $\boldsymbol{y}$. This property can be exploited for the design of output feedback to stabilize the spatially distributed tracking error $\boldsymbol{e}(z, t):=\boldsymbol{x}(z, t)-\boldsymbol{x}_{d}(z, t)$ between actual and desired profile in order to supplement the feedforward control $\boldsymbol{u}_{d}(t)$ from (2.78), which drives the system at least in a neighborhood of $\boldsymbol{x}_{d}(z, t)$. 
As a result, the error DPS for the tubular reactor (2.61)-(2.64) reads for $z \in(0,1), t>0$

$$
\frac{\partial \boldsymbol{e}}{\partial t}=S \frac{\partial^{2} \boldsymbol{e}}{\partial z^{2}}+\boldsymbol{\Psi}\left(\boldsymbol{e}+\boldsymbol{x}_{d}\right)-\left[\begin{array}{c}
L e \frac{\partial x_{1, d}}{\partial z} \\
\frac{\partial x_{2, d}}{\partial z}-\beta x_{2, d}
\end{array}\right]+\boldsymbol{\varphi}\left(\boldsymbol{x}_{d}\right)
$$

with

$$
\begin{array}{ll}
\frac{\partial \boldsymbol{e}}{\partial z}(0, t)=\mathbf{0}, & t>0 \\
P_{1} \frac{\partial \boldsymbol{e}}{\partial z}(1, t)+R_{1} \boldsymbol{e}(1, t)=Q\left(\boldsymbol{u}(t)-\boldsymbol{u}_{d}(t)\right), & t>0 \\
\boldsymbol{e}(z, 0)=\boldsymbol{e}_{0}(z), & z \in[0,1],
\end{array}
$$

whereby the right hand side of (5.53) also reflects the difference between the exact (2.66) and approximated (2.71) nonlinear source term $\Psi(\boldsymbol{x}, \partial \boldsymbol{x} / \partial z)$. Choosing the boundary control as

$$
\boldsymbol{u}(t)=\boldsymbol{u}_{d}(t)+\Delta \boldsymbol{u}(t)
$$

the output feedback control $\boldsymbol{\Delta} \boldsymbol{u}(t)$ has to be designed in order to asymptotically stabilize the error $\boldsymbol{e}(z, t)$. Various methods exist using e.g. full state feedback (Curtain and Zwart, 1995), domain-averaging (Bošković et al., 2001), or backstepping (Bošković and Krstić, 2002). Although theoretically appealing, these approaches - if at all applicable - require full or at least partial knowledge of the profile $\boldsymbol{e}(z, t)$ for all $z \in[0,1]$ and $t \in \mathbb{R}^{+}$, making the control law infinite-dimensional and usually not realizable. In order to avoid these problems and to obtain an implementable approach, classical PI-output feedback

$$
\Delta u_{i}(t)=K_{i}^{P} e_{i}(t)+K_{i}^{I} \int_{0}^{t} e_{i}(\tau) d \tau
$$

is used with $e_{i}(t)=y_{i}(t)-y_{i, d}(t)$ for $i=1,2$. For its design, either tuning rules such as the Ziegler-Nichols criteria or Lyapunov's direct method can be applied, whereby in the following the gains $K_{i}^{P}$ and $K_{i}^{I}, i=1,2$ are determined from numerical experiments using ZieglerNichols.

In summary, exploiting the property of FPSP extends the applicability of the 2DOF-approach, which amends stabilizing control (with observer) by a feedforward loop to obtain a desired tracking behavior as illustrated in Figure 5.12, to infinite-dimensional DCR-systems (Meurer and Zeitz, 2004b, 2005).

Simulation results for a parameter set corresponding to a fixed-bed tubular reactor with $P e_{1}=$ 200, $P e_{1}=100$, and $L e=500$ when applying feedforward and PI-output feedback control are depicted in Figure 5.13. In order to illustrate the robustness of the 2DOF-concept, similar to the previous section, a model error in the $D a$-number is assumed with a deviation of $+10 \%$ between simulation and control design model. Hence the initial profile $\boldsymbol{x}_{0}(z)$ also differs from the nominal profile used for feedforward control design. Nevertheless, simple PI-output feedback is sufficient to supplement the feedforward control and stabilize the system along the desired output trajectory under these severe conditions. In Figure 5.13 (top), the system outputs $\boldsymbol{y}(t)=$ 


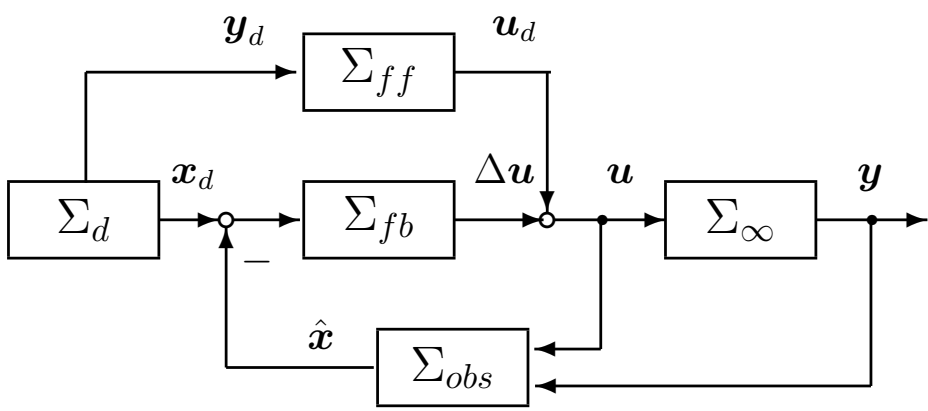

Figure 5.12: Block diagram of 2DOF-control scheme with DPS $\Sigma_{\infty}$, feedforward control $\Sigma_{f f}$, feedback control $\Sigma_{f b}$, observer $\Sigma_{o b s}$, and trajectory generator $\Sigma_{d}$ for output tracking $\boldsymbol{y}(t) \rightarrow \boldsymbol{y}_{d}(t)$.
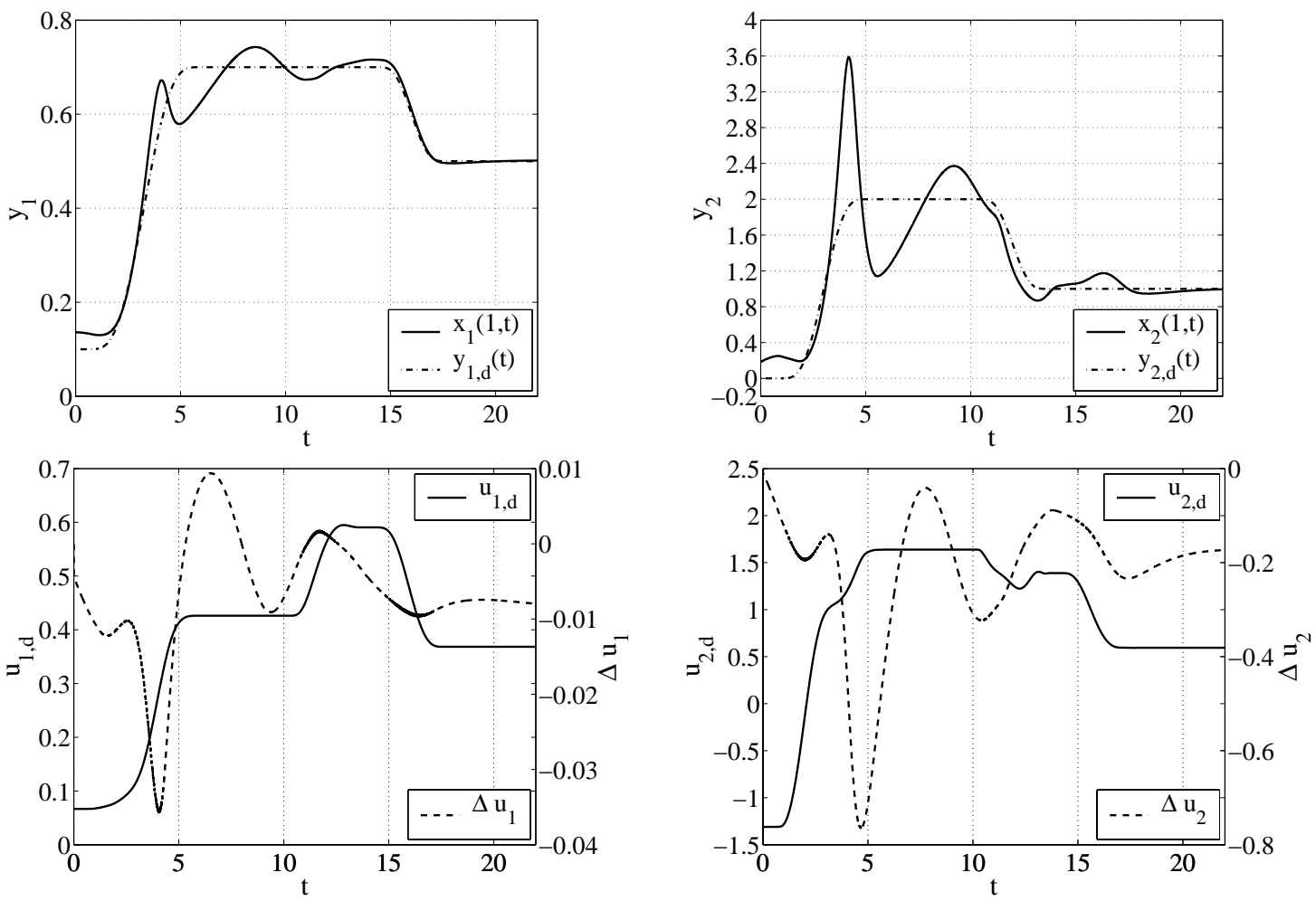

Figure 5.13: Simulation results for feedforward and PI-output feedback control of the tubular reactor model (2.61)-(2.68) with $P e_{1}=200, P e_{2}=100, L e=500$ and model error, i.e. $D a_{\text {plant }}=1.1 D a_{\text {design }}$. Top, tracking behavior for $x_{1}(0, t)$ and $x_{2}(0, t)$; bottom: comparison of feedforward $u_{i, d}(t)$ and feedback ratio $\Delta u_{i}(t), i=1,2$ in (5.57). Remaining parameters are summarized in Table D.1.

$\boldsymbol{x}(0, t)$ are shown compared to their desired values $\boldsymbol{y}_{d}(t)$. The applied controls (5.57) are split up into the feedforward part $u_{i, d}(t)$ and the PI-output feedback part $\Delta u_{i}(t), i=1,2$ (Figure 5.13 , bottom). Obviously, the feedback ratio is of magnitudes lower than the feedforward part, which is typical for the 2DOF-approach where the feedforward control ensures the desired tracking behavior and the stabilizing feedback control accounts for model errors and exogenous disturbances. 
Note that these results can be improved using more sophisticated output feedback control concepts, but illustrate the wide range of applicability of the proposed formal power series approach.

\subsection{Conclusions}

In this chapter, the property of formal power series parameterizability is applied to determine a finite-dimensional inherently flat control and observer design model for the governing infinite-dimensional systems of boundary controlled DCR-equations. The quality of the system approximation can be greatly influenced by considering appropriate summation techniques in particular the $(N, \xi)$-approximate $k$-summation - for the derivation of the design model. Based on this approximation, standard results from nonlinear control theory can be utilized to determine asymptotic tracking control with observer. Furthermore, the estimated data from the observer can be applied to spatial profile estimation during the transition process.

Stability of the closed-loop system is verified for flatness-based control of the linear heat equation. However, as is typical for feedback control of nonlinear DPS based on a finite-dimensional approximation, the theoretical verification of the stability of the combined system, i.e. DPS with feedback control and observer, is a major problem, which still lacks its solution.

Nevertheless, simulation results for the boundary controlled linear heat equation and the considered nonlinear tubular reactor model illustrate the closed-loop performance and robustness of this approach and outline further applications, such as the extension of the 2DOF-approach to infinite-dimensional systems by combining feedforward control from FPSP and stabilizing output feedback. 


\section{Chapter 6}

\section{Conclusions and outlook}

The analysis of DPS is mainly dominated by the difficulties imposed by their infinitedimensional character, which can be observed e.g. in pure, applied, and numerical mathematics. From a control engineering perspective, it is typically desired to determine the input-output behavior of a given DPS. On the other hand and as is illustrated in this thesis, the solution of the respective inverse system, namely the determination of the origin of an effect, leads to further insight in the system dynamics and directly yields the necessary rules to obtain a desired system behavior. For its design, mainly numerical discretization approaches are available, whereby in any case convergence plays the crucial role (Isakov, 1998).

This fact is reflected in this thesis, whereby a late-lumping approach based on a formal power series expansion of the system state is considered to determine an inverse system representation. It is in particular desired to provide a systematic analysis and design approach for enhanced motion planning, feedforward and flatness-based feedback tracking control design with spatial profile estimation for nonlinear parabolic systems of second-order PDEs with boundary inputs, which is suitable for numerical evaluation and computer-aided implementation. The obtained results are exemplified for several scalar examples such as the linear heat and diffusion-convection equation as well as scalar DCR-equations with polynomial nonlinearities. Furthermore, the model of a non-isothermal fixed-bed tubular reactor is studied within several branches of operation.

The proposed approach thereby comprises four steps: formal power series parameterization, introduction of suitable summation methods, and their combination to feedforward as well as feedback tracking control design.

At first, the notion of formal power series parameterizability is introduced, which formally allows to overcome the restriction to uniformly convergent power series as e.g. considered by Laroche et al. (2000); Lynch and Rudolph (2002); Rudolph (2003a).

However, an algebraic structure has to be identified with the considered space of formal power series, which leads to summability methods with the focus on $k$-summability. This in particular enables to deal with certain divergent power series and allows to compute their sum (in the sense of the summation method). Since the corresponding theory is far from complete for formal 
solutions to PDEs (Balser and Kostov, 2003) and since especially nonlinear problems only allow to determine a finite number of series coefficients, certain modifications of the summability approach are required in order to accurately approximate the sum of a given slowly converging or possibly diverging series from a given finite number of series coefficients. Within this framework of generalized sequence transformations, a variant of $k$-summation is introduced, namely the $(N, \xi)$-approximate $k$-summation (Meurer and Zeitz, 2004a; Wagner et al., 2004).

The novel combination of FPSP and appropriate GSTs provides a widely applicable approach for the design of feedforward tracking control, which is confirmed by numerical studies for formal parameterizations of the linear heat equation, the diffusion-convection equation, and the model of a non-isothermal tubular reactor.

In addition, the re-interpretation of FPSP allows to determine an inherently flat finitedimensional approximation of the governing infinite-dimensional DPS as a system of ODEs in the coefficients of the formal power series. Based on this finite-dimensional model, standard flatness-based methods can be utilized for tracking control design with observer. Moreover, the observer data can be directly applied to spatial profile estimation e.g. for monitoring purposes throughout the transition process. Thereby, application of the $(N, \xi)$-approximate $k$-summation yields a significant improvement of the results, as is illustrated in numerical simulations for tracking control of the linear heat equation and the model of a non-isothermal tubular reactor.

In particular it should be pointed out that the proposed feedforward and feedback design approach directly accounts for a computer-aided implementation using computer-algebrasystems.

Further perspectives of the formal power series approach concern the study of more general system structures. Initial results towards a generalization can be found e.g. in (Schaum, 2005), where the model of a fixed-bed tubular reactor with cooling jacket is considered. Thereby, results for both concurrent as well as countercurrent flow of the cooling medium with respect to the flow inside the reactor are available.

On the other hand, applications typically require to satisfy certain input constraints due to physical limitations, e.g. limited heating power or inlet concentration. These restrictions can be similarly taken into account for formal power series parameterizable linear and nonlinear parabolic DPS as initially illustrated in (Meurer et al., 2005) for the scalar DCR-equations with boundary input. An extension of the thereby considered approach seems desirable for systems modeled by MIMO sets of DCR-equations, such as fixed-bed tubular reactors. 


\section{Appendix A}

\section{Mathematical glossary}

\section{A.1 Analysis}

Theorem A.1 (Cauchy-Hadamard). The radius of convergence $\rho$ of a power series $\sum_{n} a_{n} z^{n}$ is given by

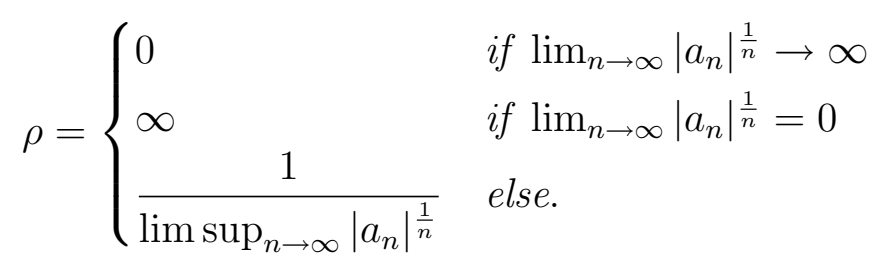

Here $\lim \sup _{n \rightarrow \infty}\left|a_{n}\right|^{\frac{1}{n}}$ denotes the upper accumulation limit of the sequence $\left|a_{n}\right|^{\frac{1}{n}}$ (see e.g. Heuser (1993)).

Lemma A.1 (Stirling's formula). For $n \gg 1$ it holds that

$$
n ! \sim \sqrt{2 \pi} n^{n+\frac{1}{2}} e^{-n}
$$

where $\sim$ is used to indicate that the ratio of the two sides goes to 1 as $n \rightarrow \infty$, i.e.

$$
\lim _{n \rightarrow \infty} \frac{n !}{\sqrt{2 \pi} n^{n+\frac{1}{2}} e^{-n}}=1 .
$$

See e.g. Heuser (1993) for a proof.

Lemma A.2. $\sum_{k} a_{k}^{\rho} \leq\left(\sum_{k} a_{k}\right)^{\rho}, \quad \rho \geq 1, a_{k} \geq 0$

Lemma A.3.

$$
\frac{i ! j !(i+j+l+1) !}{(i+j+1) !}=\sum_{r=0}^{l}\left(\begin{array}{l}
l \\
r
\end{array}\right)(j+r) !(i+l-r) !, \quad i, j, l \geq 0
$$

Proofs can be found e.g. in Lynch and Rudolph (2002). 


\section{A.2 Complex analysis}

In the following, $\mathbb{E}$ denotes a Banach space over the complex number field $\mathbb{C}$ and $G$ be some fixed region in $\mathbb{C}$.

Definition A.1 (Boundedness). The function $x$ is bounded at the origin, if for every closed subsector $\bar{S}$ of $\Omega$ there exists a positive real constant $c$ depending on $\bar{S}$, such that $\|x(z)\| \leq c$ for $z \in \bar{S}$.

Definition A.2 (Continuity). The function $x$ is continuous at the origin, if an element of $\mathbb{E}$, denoted by $x(0)$ exists, such that

$$
x(z) \longrightarrow x(0), \quad \Omega \ni z \rightarrow 0,
$$

with convergence being uniform in every closed subsector, meaning that for every $\bar{S} \subset \Omega$ and every $\epsilon>0$ there exists $\rho>0$ so that $\|x(z)-x(0)\| \leq \epsilon$ for $z \in \bar{S}$ with $|z|<\rho$. 


\section{Appendix B}

\section{Smooth functions with compact support}

For the considered class of formal power series parameterizable DCR-systems with boundary inputs, trajectory planning is based on $\mathcal{C}^{\infty}$-functions having compact support in order to realize desired transitions within finite time-intervals. Exemplarily, the following function $\Phi_{\gamma, T}: \mathbb{R}^{+} \rightarrow$ $\mathbb{R}$, i.e.

$$
\Phi_{\gamma, T}(t)= \begin{cases}0 & \text { if } t \leq 0 \\ 1 & \text { if } t \geq T \\ \frac{\int_{0}^{t} \phi_{\gamma, T}(\tau) d \tau}{\int_{0}^{T} \phi_{\gamma, T}(\tau) d \tau} & \text { if } t \in(0, T)\end{cases}
$$

with

$$
\phi_{\gamma, T}(t)= \begin{cases}0 & \text { if } t \notin(0, T) \\ \exp \left(\frac{-1}{\left[\left(1-\frac{t}{T}\right) \frac{t}{T}\right]^{\gamma}}\right) & \text { if } t \in(0, T) .\end{cases}
$$

is used for motion planning as proposed in Fliess et al. (1997). Alternatively, quite similar functions can be e.g. found in Petit (2002); Laroche (2000); Rudolph (2003a,b); Rudolph et al. (2003).

The function $\Phi_{\gamma, T}(t)$ is non-analytic at $t \in\{0, T\}$, i.e. for $n>0$ the derivatives satisfy $\Phi_{\gamma, T}^{(n)}(t)=0$ for $t \leq 0$ or $t \geq T$ but $\Phi_{\gamma, T}^{(n)}(t) \neq 0$ for $t \in(0, T)$. In particular, its Gevrey order (3.14) can be determined as $\alpha=1+\frac{1}{\gamma}$ (Lynch and Rudolph, 2002). Figure B.1 shows the behavior of $\Phi_{\gamma, T}(t)$ when varying the parameter $\gamma$ and hence the Gevrey order $\alpha$. Obviously, the curve flattens with decreasing values of $\gamma$, or respectively increasing Gevrey order $\alpha$. On the other hand, opposing behavior is observed for the derivatives of $\Phi_{\gamma, T}(t)$ as depicted in Figure B.2. There, the derivatives $\Phi_{\gamma, T}^{(n)}(t)$ for $n=1,3,5,10$ are shown over $t$ on a semi-logarithmic scale. Clearly, for $\gamma=2$ the maximum values are located in the interior of the domain $t \in[0, T]$, but move to the boundaries at $t \in\{0, T\}$ when decreasing $\gamma$. In addition, a significant increase in the amplitudes $\left|\Phi_{\gamma, T}^{(n)}(t)\right|$ can be observed, which is directly related to the Gevrey order $\alpha=1+\frac{1}{\gamma}$ of $\Phi_{\gamma, T}(t)$, i.e. $\sup _{t \in[0, T]}\left|\Phi_{\gamma, T}^{(n)}(t)\right| \leq \frac{M}{R^{n}}(n !)^{\alpha}$ - see also Def. 3.11 . 


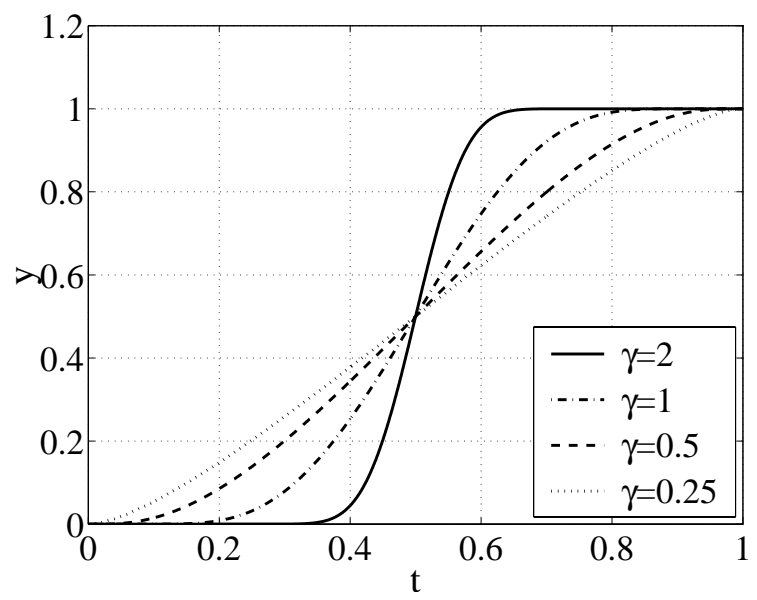

Figure B.1: $\mathcal{C}^{\infty}$-function $\Phi_{\gamma, T}(t)$ from (B.1) with $T=1$ and varied $\gamma \in\{2,1,0.5,0.25\}$. The respective Gevrey order is thereby given by $\alpha=1+\frac{1}{\gamma} \in\{1.5,2,3,5\}$.
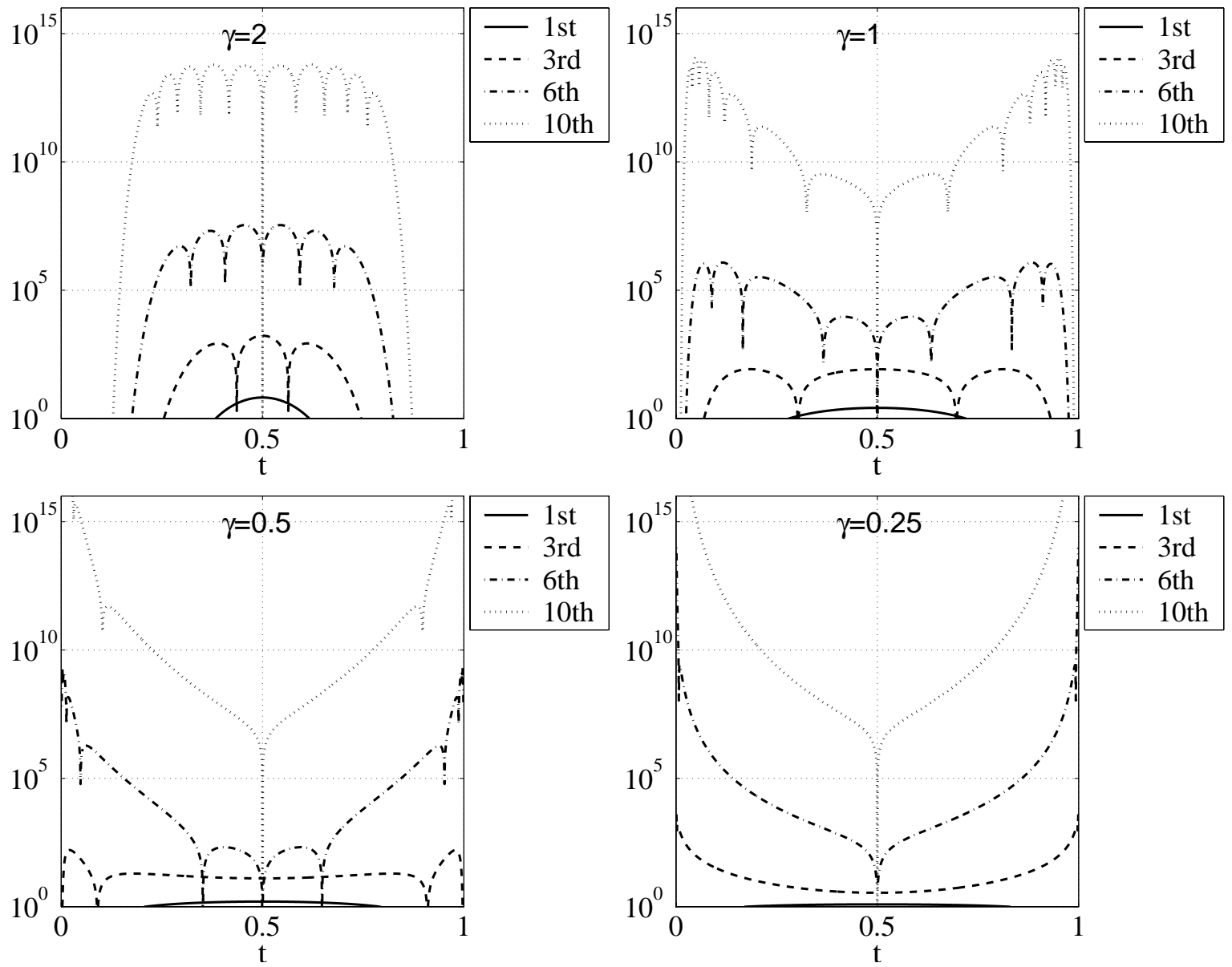

Figure B.2: Derivatives of $\Phi_{\gamma, T}(t)$ from (B.1) on a semi-logarithmic scale with $T=1$ and varied $\gamma \in\{2,1,0.5,0.25\}$. The respective Gevrey order is thereby given by $\alpha=1+\frac{1}{\gamma} \in\{1.5,2,3,5\}$. 


\section{Appendix C}

\section{An algorithm for the efficient evaluation of nonlinear differential recurrence relations}

In the following, a pattern-based symbolic algorithm for the use with computer-algebrasystems (CASs) is summarized, which allows to exactly evaluate a given second-order differential recursion in $K$-equations

$$
\begin{aligned}
\boldsymbol{\eta}_{n+2}(t) & =\boldsymbol{\mathcal { R }}\left(\boldsymbol{\eta}_{n+1}(t), \boldsymbol{\eta}_{n}(t), \frac{d \boldsymbol{\eta}_{n}(t)}{d t}\right), \quad n \in \mathbb{N}_{0}, \\
\boldsymbol{\eta}_{0} & =\boldsymbol{\alpha}(t), \\
\boldsymbol{\eta}_{1} & =\boldsymbol{\beta}(t)
\end{aligned}
$$

up to a high number of coefficients $\boldsymbol{\eta}_{n}(t), n \gg 1$. The idea is based on sequential evaluation of the recursion combined with pattern-based routines to simplify the results.

Figure C.1 schematically summarizes the algorithm in standard MATHEMATICA notation. It is initialized by Eqns. (C.1)-(C.3) and user-defined number of desired coefficients $n_{\max } \in \mathbb{N}$. This directly allows to determine the number of derivatives of each coefficient necessary to compute the coefficients $\boldsymbol{\eta}_{n}(t)$ for $n=0,1, \ldots, n_{\max }$. The corresponding computations are performed within the 'inner loop' depicted in Figure C.1. Thereby, the list 'repList' of derivatives $\boldsymbol{\eta}_{n, m}$, $m=0,1, \ldots, n_{\max }-\frac{n-n \bmod 2}{2}$ of the $n$-th coefficient $\boldsymbol{\eta}_{n}$ is build-up, with any appearing derivative of the coefficients $d^{m} \boldsymbol{\eta}_{j}(t) / d t^{m}$ with $j<n$ being substituted by the state $\boldsymbol{\eta}_{j, m}$. The respective rule is applied using 'repRule'. As a result of the evaluation of the 'outer loop', a table of equations for the states $\boldsymbol{\eta}_{n, m}, n=0,1, \ldots, n_{\max }, m=0,1, \ldots, n_{\max }-\frac{n-n \bmod 2}{2}$ is obtained. The great advantage of this approach is related to the introduction of intermediate states, namely $\boldsymbol{\eta}_{n, m}$ with $m>0$, such that the resulting equations can be evaluated in a 'top-down' procedure with rather simple expressions for each state. In particular since only symbolic computations are performed using CASs, the determined solution is exact. An efficient 


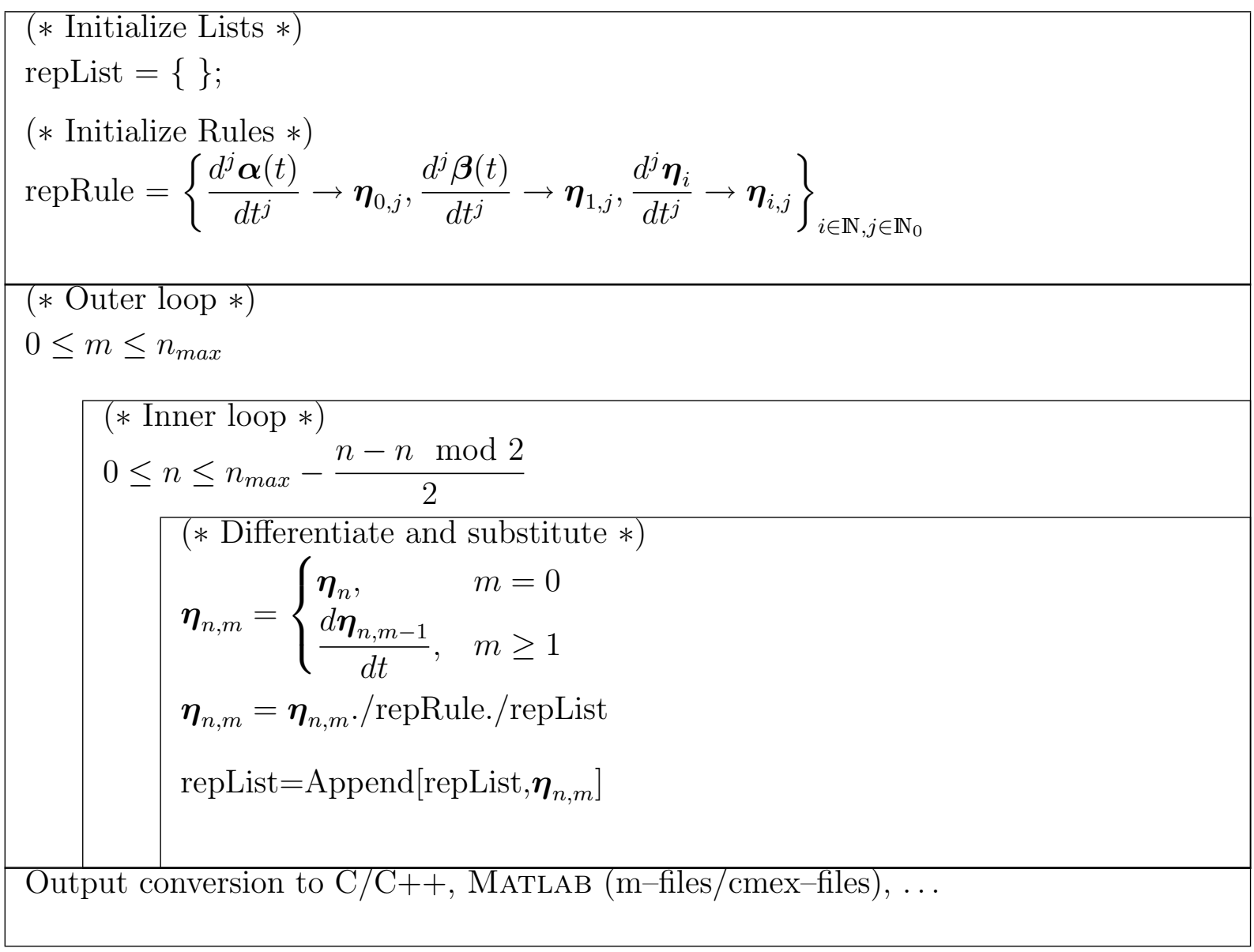

Figure C.1: Algorithm for the pattern-based sequential evaluation of the second-order differential recursion (C.1)-(C.3). Thereby Mathematica notation was used to express comments '(*...*)', rules ' $a \rightarrow b$ ', and replacement operations './'.

numerical evaluation is possible by MATHEMATICA conversion routines to MATLAB m-files, Matlab cmex-files, or C/C++ codes (Meurer, 2003b). 


\section{Appendix D}

\section{Lemmas and parameters for the non-isothermal tubular reactor model}

\section{D.1 Lemma on recursion}

Theorem D.1. The coefficients of the differential recursion for the tubular reactor

$$
\begin{aligned}
\hat{x}_{1, n+2} & =\frac{P e_{1}}{(n+2)(n+1)}\left(\frac{1}{L e} \frac{d \hat{x}_{1, n}}{d t}-(n+1) \hat{x}_{1, n+1}-\frac{1}{L e} \hat{\varphi}_{1, n}\left(\underline{\hat{x}}_{n+1}\right)\right) \\
\hat{x}_{2, n+2} & =\frac{P e_{2}}{(n+2)(n+1)}\left(\frac{d \hat{x}_{2, n}}{d t}-(n+1) \hat{x}_{2, n+1}+\beta \hat{x}_{2, n}-\hat{\varphi}_{2, n}\left(\underline{\hat{\boldsymbol{x}}}_{n+1}\right)\right) \\
\hat{x}_{i, 0} & =y_{i}(t), \quad i=1,2 \\
\hat{x}_{i, 1} & =0
\end{aligned}
$$

with $n \in \mathbb{N}_{0}$ satisfy

$$
\begin{aligned}
\hat{x}_{i, 2 j} & =\boldsymbol{\vartheta}_{i, 2 j}\left(\underline{\hat{\boldsymbol{x}}}_{2 j-2}^{\prime}, \frac{d \hat{x}_{i, 2 j-2}}{d t}\right) \\
\hat{x}_{i, 2 j+1} & =\boldsymbol{\vartheta}_{i, 2 j+1}\left(\underline{\hat{\boldsymbol{x}}}_{2 j}^{\prime}\right)
\end{aligned}
$$

for $i=1,2$ and $j \in \mathbb{N}$ where $\underline{\hat{x}}_{2 j}^{\prime}=\left[\hat{\boldsymbol{x}}_{0}^{T}, \hat{\boldsymbol{x}}_{2}^{T}, \ldots, \hat{\boldsymbol{x}}_{2 j}^{T}\right]^{T}$.

Proof. The proof follows by induction. Assume (D.1), (D.2) hold for $j \in 1,2, \ldots, m$. This implies for $n=2 m$ in the differential recursion

$$
\begin{aligned}
\hat{x}_{1,2 m+2}= & \frac{P e_{1}}{(2 m+2)(2 m+1)}\left(\frac{1}{L e} \frac{d \hat{x}_{1,2 m}}{d t}-(2 m+1) \hat{x}_{1,2 m+1}-\frac{1}{L e} \hat{\varphi}_{1,2 m}\left(\underline{\hat{\boldsymbol{x}}}_{2 m+1}\right)\right) \\
= & \frac{P e_{1}}{(2 m+2)(2 m+1)}\left(\frac{1}{L e} \frac{d \hat{x}_{1,2 m}}{d t}-(2 m+1) \boldsymbol{\vartheta}_{i, 2 m+1}\left(\underline{\hat{x}}_{2 m}^{\prime}\right)\right. \\
& \left.-\frac{1}{L e} \hat{\varphi}_{1,2 m}\left(\left[\hat{\boldsymbol{x}}_{0}^{T}, \mathbf{0}^{T}, \hat{\boldsymbol{x}}_{2}^{T}, \boldsymbol{\vartheta}_{i, 3}^{T}\left(\underline{\hat{\boldsymbol{x}}}_{2}^{\prime}\right), \ldots, \boldsymbol{\vartheta}_{i, 2 m+1}^{T}\left(\underline{\hat{\boldsymbol{x}}}_{2 m}^{\prime}\right)\right]^{T}\right)\right) \\
= & \boldsymbol{\vartheta}_{1,2 m+2}\left(\underline{\hat{\boldsymbol{x}}}_{2 m}^{\prime}, \frac{d \hat{x}_{i, 2 m}}{d t}\right) .
\end{aligned}
$$


Furthermore, it follows for $n=2 m+1$

$$
\begin{aligned}
\hat{x}_{1,2 m+3}= & \frac{P e_{1}}{(2 m+3)(2 m+2)}\left(\frac{1}{L e} \frac{d \hat{x}_{1,2 m+1}}{d t}-(2 m+2) \hat{x}_{1,2 m+2}-\frac{1}{L e} \hat{\varphi}_{1,2 m+1}\left(\underline{\boldsymbol{x}}_{2 m+2}\right)\right) \\
& \stackrel{(D .2)}{=} \frac{P e_{1}}{(2 m+3)(2 m+2)}\left(\frac{1}{L e} \sum_{l=0}^{m} \frac{\partial \boldsymbol{\vartheta}_{1,2 m+1}}{\partial \hat{\boldsymbol{x}}_{2 l}} \frac{d \hat{\boldsymbol{x}}_{2 l}}{d t}-(2 m+2) \hat{x}_{1,2 m+2}\right. \\
& \left.-\frac{1}{L e} \hat{\varphi}_{1,2 m+1}\left(\left[\hat{\boldsymbol{x}}_{0}^{T}, \mathbf{0}^{T}, \hat{\boldsymbol{x}}_{2}^{T}, \boldsymbol{\vartheta}_{i, 3}^{T}\left(\underline{\hat{\boldsymbol{x}}}_{2}^{\prime}\right), \ldots, \underline{\hat{\boldsymbol{x}}}_{2 m+2}^{T}\right]^{T}\right)\right) .
\end{aligned}
$$

Since the differential recursion is linear in the time-derivative, (D.1) can be explicitly solved for any $d \hat{x}_{i, 2 l} / d t=\Theta_{i, 2 l}\left(\underline{\hat{x}}_{2 l}^{\prime}, \hat{x}_{i, 2 l+2}\right), i=1,2$. Hence the latter equation reduces to

$$
\hat{x}_{1,2 m+3}=\boldsymbol{\vartheta}_{1,2 m+3}\left(\underline{\hat{\boldsymbol{x}}}_{2 m+2}^{\prime}\right) .
$$

Similar calculations for $\hat{x}_{2,2 m+2}$ and $\hat{x}_{2,2 m+3}$ complete the proof.

\section{D.2 Model and simulation parameters}

The dimensionless parameters of the tubular reactor model (2.61)-(2.68) are chosen as follows: $B=12.0, D a=0.11, \beta=1.5, \sigma=20.0, x_{2 w}=0$. Peclet numbers $P e_{1}, P e_{2}$ and Lewis number $L e$ are varied and summarized in the respective sections.

The desired trajectory (4.5) is parameterized by $y_{1, S}^{0}=0.1, y_{1, S}^{1}=0.7, y_{1, S}^{2}=0.5, y_{2, S}^{0}=0$, $y_{2, S}^{1}=2, y_{2, S}^{2}=1, \gamma_{1}^{1}=\gamma_{1}^{2}=\gamma_{2}^{1}=\gamma_{2}^{2}=1, T_{1}^{0}=0, T_{1}^{1}=6.5, T_{1}^{2}=14, T_{1}^{3}=18, T_{2}^{0}=0.5$, $T_{2}^{1}=4.5, T_{2}^{2}=10, T_{2}^{3}=14$.

The following table summarizes the used Peclet $P e_{1}, P e_{2}$, Lewis numbers $L e$ and summation parameters $\xi_{1}, k_{1}, \xi_{2}, k_{2}$ in the consecutive figures. Thereby, the optimization procedure introduced in Section 4.4.1.2 was applied to determine the summation parameters.

Table D.1: Summary of varied model parameters and summation parameters in the consecutive figures.

\begin{tabular}{c||c|c|c|c|c|c|c|} 
Fig. & $P e_{1}$ & $P e_{2}$ & $L e$ & $\xi_{1}$ & $k_{1}$ & $\xi_{2}$ & $k_{2}$ \\
\hline \hline \multirow{2}{*}{4.9} & 6 & 6 & 1 & 25 & $1 / 2$ & 3900 & $1 / 3$ \\
\cline { 2 - 8 } & 10 & 10 & 1 & 25 & $1 / 2$ & 3900 & 0.339 \\
\cline { 2 - 8 } & 200 & 100 & 500 & 35000 & 0.2041 & 22 & 0.4739 \\
\hline 5.11 & 6 & 6 & 1 & 5 & 1 & 5 & 1 \\
\hline 5.13 & 200 & 100 & 500 & 35000 & 0.2041 & 22 & 0.4739 \\
\hline
\end{tabular}




\section{Bibliography}

C. Antoniades and P.D. Christofides. Studies on nonlinear dynamics and control of a tubular reactor with recycle. Nonlinear Analysis, 47:5933-5944, 2001.

Y. Aoustin, M. Fliess, H. Mounier, P. Rouchon, and J. Rudolph. Theory and practice in the motion planning and control of a flexible robot arm using Mikusiński operators. In: Proc. 5th IFAC Symposium on Robot Control, pp. 287-293, Nantes, France, 1997.

J.A. Atwell and B.B. King. Proper orthogonal decompostion for reduced basis feedback controllers for parabolic equations. Mathematics and Computer Modelling, 33:1-19, 2001.

M.J. Balas. Modal control of certain flexible dynamic systems. SIAM J. Control Optim., 16(3): 450-462, 1978.

M.J. Balas. Towards a (more) practical control theory for distributed parameter systems. In: C.T. Leondes (edit.), Control and Dynamic Systems: Advances in Theory and Applications, volume 18. 1982a.

M.J. Balas. Trends in large space structure control theory: Fondest hope, wildest dreams. IEEE Trans. Automat. Control, AC-27(3):522-535, 1982b.

M.J. Balas. The Galerkin Method and Feedback Control of Linear Distributed Parameter Systems. J. Math. Anal. Appl., 91:527-546, 1983.

W. Balser. From Divergent Power Series to Analytic Functions. Springer, 1994.

W. Balser. Divergent solutions of the heat equation: on an article of Lutz, Miyake and Schäfke. Pacific J. Math., 188(1):53-63, 1999.

W. Balser. Formal power series and linear systems of meromorphic ordinary differential equations. Springer-Verlag, 2000.

W. Balser. Summability of formal power series solutions of ordinary and partial differential equations. Functional Diff. Eq., 8:11-24, 2001.

W. Balser. Power series solutions of the inhomogeneous heat equation. Preprint, 2003.

W. Balser. Personal communication, 2003/2004.

W. Balser. Formal power series solutions of the heat equation in one spatial variable. Preprint, 2004. 
W. Balser and R.W. Braun. Power Series Methods and Multisummability. Math. Nachr., 212:37-50, 2000.

W. Balser and V. Kostov. Recent progress in the theory of formal solutions for ODE and PDE. Applied Mathematics and Computation, 141:113-123, 2003.

W. Balser and S. Malek. Formal solutions of the complex heat equation in higher spatial dimensions. Preprint, 2003.

W. Balser and M. Miyake. Summability of formal solutions of certain partial differential equations. Acta Sci. Math (Szeged), 65:543-551, 1999.

M. Berezowski. Effect of the delay time on the generation of chaos in continuous systems. one dimensional model. two dimensional model - tubular chemical reactor with recycle. Chaos, Solitons and Fractals, 12:83-89, 2001.

R.P. Boas. Entire functions. Academic Press, New York, 1954.

E. Borel. Mémoires sur les séries diverentes. Annales Scientifiques de l'Ecole Normale Superieure, 16: 9-136, 1899.

E. Borel. Leçons sur les séries diverentes. Gauthier-Villar, 1928.

D.M. Bošković and M. Krstić. Backstepping control of chemical tubular reactors. Comp. Chem. Eng., 26:1077-1085, 2002.

D.M. Bošković, M. Krstić, and W. Liu. Boundary control of an unstable heat equation via measurement of domain-averaged temperature. IEEE Trans. Automat. Control, 46(12):2022-2028, 2001.

C. Brezinski. Accéleration de la convergence en analyse numérique. Lecture Notes in Mathematics. Springer-Verlag, Berlin, 1977.

I.N. Bronstein, K.A. Semendjajew, G. Musiol, and H. Mühlig. Taschenbuch der Mathematik. Verlag Harri Deutsch, 1997.

A.G. Butkovsky. Distributed Control Systems. Elsevier, 1969.

C.I. Byrnes, D.S. Gilliam, A. Isidori, and V.I. Shubov. Static and Dynamic Controllers for Boundary Controlled Distributed Parameter Systems. In: Proc. (CD-Rom) 43rd IEEE Conference on Decision and Control (CDC), pp. 3324-3325, Bahamas, Dec. 14-17 2004.

C.I. Byrnes, I.G. Laukó, D.S. Gilliam, and V.I. Shubov. Output Regulation for Linear Distributed Parameter Systems. IEEE Trans. Automat. Control, 40(12):2236-2252, 2000.

P.D. Christofides. Nonlinear and Robust Control of PDE Systems. Birkhäuser, 2001.

R.F. Curtain. Pole Assignment for Distributed Systems by Finite-Dimensional Control. Automatica, 21(1):57-67, 1985 . 
R.F. Curtain and H.J. Zwart. An Introduction to Infinite-Dimensional Linear Systems Theory. Texts in Applied Mathematics 21. Springer-Verlag New York, 1995.

A. Doubova, E. Fernández-Cara, M. González-Burgos, and E. Zuazua. On the controllability of parabolic systems with a nonlinear term involving the state and the gradient. SIAM J. Control Optim., 41(3):798-819, 2002.

W.B. Dunbar, N. Petit, P. Rouchon, and P. Martin. Motion Planning for a nonlinear Stefan Problem. ESAIM-COCV, 9:275-296, 2003.

H.O. Fattorini. Boundary control systems. SIAM J. Control, 6(3):349-388, 1968.

M. Fliess, J. Lévine, P. Martin, and P. Rouchon. Flatness and defect of non-linear systems: introductory theory and examples. Internat. J. Control, 61:1327-1361, 1995.

M. Fliess, H. Mounier, P. Rouchon, and J. Rudolph. Systèmes linéaires sur les opérateurs de Mikusiński et commande d'une poutre flexible. ESAIM Proceedings, 2:183-193, 1997.

M. Fliess, H. Mounier, P. Rouchon, and J. Rudolph. Controlling the transient of a chemical reactor: a distributed parameter approach. In: Proc. Computational Engineering in Systems Application IMACS Multiconference (CESA'98), Hammamet, Tunisia, 1998a.

M. Fliess, H. Mounier, P. Rouchon, and J. Rudolph. A distributed parameter approach to the control of a tubular reactor: a multi-variable case. In: Proc. 37th Conference on Decision and Control, pp. 439-442, Tampa, FL, USA, 1998b.

E. Freund. Zeitvariable Mehrgrößensysteme. Springer-Verlag, 1971.

A.V. Fursikov. Optimal control of distributed systems. Theory and applications. AMS, 1999.

C. Georgakis, R. Aris, and N.R. Amundson. Studies in the control of tubular reactors - I. Chem. Eng. Sci., 32:1359-1369, 1977a.

C. Georgakis, R. Aris, and N.R. Amundson. Studies in the control of tubular reactors - II. Chem. Eng. Sci., 32:1371-1379, 1977b.

C. Georgakis, R. Aris, and N.R. Amundson. Studies in the control of tubular reactors - III. Chem. Eng. Sci., 32:1381-1387, 1977c.

R. Gérard and H. Tahara. Formal power series solutions of nonlinear first order partial differential equations. Funkcialaj Ekvacioj, 41:133-166, 1998.

M. Gevrey. La nature analytique des solutions des équation aux dérivées partielles. Annales Scientifiques de l'Ecole Normale Superieure, 25:129-190, 1918.

W. Haack and W. Wendland. Vorlesungen über Partielle und Pfaffsche Differentialgleichungen. Birkhäuser Verlag Basel \& Stuttgart, 1969.

G.H. Hardy. Divergent Series. Oxford at the Clarendon Press, 1. edition, 1949. 
H. Heuser. Lehrbuch der Analysis, Teil 1. B.G. Teubner Stuttgart, 10 edition, 1993.

M. Hibino. Gevrey asymptotic theory for singular first order linear partial differential equations of nilpotent type - part ii. Publ. Res. Inst. Math. Sci., 37(4):579-614, 2001.

M. Hibino. Gevrey asymptotic theory for singular first order linear partial differential equations of nilpotent type - part i. Comm. Pure Appl. Analysis, 2(2):211-231, 2003.

I.M. Horowitz. Synthesis of Feedback Systems. Academic Press: New York, 1963.

C. Hua and L. Rodino. General theory of PDE and Gevrey classes. In: Q.Min-You and L.Rodino (eds.), General Theory of PDEs and Microlocal Analysis, pp. 6-81. Addison Wesley, 1996.

V. Isakov. Inverse Problems for Partial Differential Equations. Applied Mathematical Sciences 127. Springer-Verlag New York, 1998.

K.F. Jensen and W.H. Ray. The bifurcation behavior of tubular reactors. Chem. Eng. Sci., 37(2): 199-222, 1982 .

K. Knopp. Theorie und Anwendung der unendlichen Reihen. Grundlehren der mathematischen Wissenschaften in Einzeldarstellungen. Springer, 1964.

S. Lang. Algebra. Addison-Wesley, 3rd edition, 1993.

B. Laroche. Extension de la notion de platitude à des systèmes décrits par des équations aux dérivées partielles linéaires. PhD thesis, École Nationale Supérieure des Mines de Paris, 2000.

B. Laroche and P. Martin. Motion planning for a 1-D diffusion equation using brunovsky-like decomposition. In: Proc. 14th Int. Symposium on Mathematical Theory of Networks and Systems (MTNS), Perpignan, France, 2000.

B. Laroche, P. Martin, and P. Rouchon. Motion planning for a class of partial differential equations with boundary control. In: Proc. 37th Conference on Decision and Control, pp. 3494-3497, Tampa, FL, USA, 1998.

B. Laroche, P. Martin, and P. Rouchon. Motion planning for the heat equation. Int. J. Robust Nonlinear Control, 10:629-643, 2000.

I. Lasiecka and R. Triggiani. Stabilization and structural assignment of Dirichlet boundary feedback parabolic equations. SIAM J. Control Optim., 21(5):766-803, 1983.

D. Levin. Development of non-linear transformations for improving convergence of sequences. Int. J. Comput. Math. B, 3:371-388, 1973.

J.L. Lions. Optimal Control of Systems Governed by Partial Differential Equations. Springer, 1971.

J. Lunze. Regelungstechnik II. Springer-Verlag, 2005.

Z.H. Luo, B.Z. Guo, and O.Morgul. Stability and Stabilization of Infinite Dimensional Systems with Applications. Springer-Verlag, London, 1999. 
D.A. Lutz, M. Miyake, and R. Schäfke. On the Borel summability of divergent solutions of the heat equation. Manuskript, 1996.

A.F. Lynch and J. Rudolph. Flachheitsbasierte Randsteuerung parabolischer Systeme mit verteilten Parametern. at-Automatisierungstechnik, 48(10):478-486, 2000a.

A.F. Lynch and J. Rudolph. Flatness-based boundary control of a nonlinear parabolic equation modelling a tubular reactor. In: A. Isidori, F. Lamnabhi-Lagarrique, and W. Respondek (eds.), Nonlinear Control in the Year 2000, volume 2 of Lecture Notes in Control and Information Science, pp. 45-54. Springer-Verlag, 2000b.

A.F. Lynch and J. Rudolph. Flatness-based boundary control of coupled nonlinear PDEs modelling a tubular reactor. In: Proc. International Symposium on Nonlinear Theory and its Applications NOLTA2000, pp. 641-644, Dresden, Germany, 2000c.

A.F. Lynch and J. Rudolph. Flatness-based boundary control of a class of quasilinear parabolic distributed parameter systems. Internat. J. Control, 75(15):1219-1230, 2002.

B. Malgrange. Sommation des séries divergentes. Expositiones Mathematicae, 13:163-222, 1995.

P. Martin, R. Murray, and P. Rouchon. Flat systems. In: 4th European Control Conference (ECC), Plenary Lectures and Mini-Courses, pp. 211-264, Brussels, Belgium, 1997.

T. Meurer, J. Becker, and M. Zeitz. Flatness-based feedback tracking control of a distributed parameter tubular reactor model. In: Proc. (CD-ROM) European Control Conference (ECC), Cambridge, UK, Sep. 1-4 2003.

T. Meurer, K. Graichen, and M. Zeitz. Motion planning and feedforward control for distributed parameter systems under input constraints. In: Proc. (CD-Rom) IFAC World Congress, Prague (CZ), July 4-8 2005.

T. Meurer and M. Zeitz. A modal approach to flatness-based control of flexible structures. Proc. Appl. Math. Mech. (PAMM), 4(1):133-134, 2003a.

T. Meurer and M. Zeitz. A Novel Design Approach to Flatness-Based Feedback Boundary Control of Nonlinear Reaction-Diffusion Systems with Distributed Parameters. In: W. Kang, C. Borges, and M. Xiao (eds.), New Trends in Nonlinear Dynamics and Control, Lecture Notes in Control and Information Sciences, pp. 221-236. Springer-Verlag, New York, 2003b.

T. Meurer and M. Zeitz. Flatness-based feedback control of diffusion-convection-reaction systems via $k$-summable power series. In: Proc. 6th IFAC Symposium "Nonlinear Control Systems" (NOLCOS 2004), pp. 191-196, Stuttgart, Germany, 2004a.

T. Meurer and M. Zeitz. Two-degree-of-freedom tracking control design for boundary controlled distributed parameter systems using formal power series. In: Proc. (CD-Rom) 43rd IEEE Conference on Decision and Control (CDC), pp. 3307-3312, Bahamas, Dec. 14-17 2004b.

T. Meurer and M. Zeitz. Feedforward and feedback tracking control of nonlinear diffusion-convectionreaction systems using summability methods. Ind. Eng. Chem. Res., 44:2532-2548, 2005. 
J. Mikusiński. Operational Calculus, volume 1. Pergamon, Oxford \& PWN, 1983.

J. Mikusiński and T.K. Boehme. Operational Calculus, volume 2. Pergamon, Oxford \& PWN, 1987.

K.S. Miller. The Mittag-Leffler and Related Functions. Integral Transf. Spec. Funct., 1:41-49, 1993.

M.G. Mittag-Leffler. Sur la nouvelle fonction $E_{\alpha}(x)$. Comptes Rendus Acad. Sci. Paris, 137:554-558, 1903.

T. Nambu. Feedback Stabilization for Distributed Parameter Systems of Parabolic Type. J. Diff. Equations, 33:167-188, 1979.

T. Nambu. On the Stabilization of Diffusion Equations: Boundary Observation and Feedback. $J$. Diff. Equations, 52:204-233, 1984.

F. Nevanlinna. Zur Theorie asymptotischer Potenzreihen. Ann. Acad. Sci. Fenn. Ser. A, 12:1-81, 1919.

F. Ollivier and A. Sedoglavic. A generalization of flatness to nonlinear systems of partial differential equations. Applications to the command of a flexible rod. In: Proc. 5th IFAC Symposium "Nonlinear Control Systems" (NOLCOS 2001), pp. 196-200, Saint-Petersburg, Russia, 2001.

S. Ouchi. Formal solutions with Gevrey type estimates of nonlinear partial differential equations. $J$. Math. Sci. Univ. Tokyo, 1:205-237, 1994.

V. Panfilov and M. Sheintuch. Control strategies for front stabilization in a tubular reactor model. AIChE, 47(1):187-196, 2001.

N. Petit. Systèmes à retards. Platitude en génie des procédés et contrôle de certaines équations des ondes. Thèse de doctorat, École des Mines de Paris, 2002.

J.-P. Ramis. Les seriés k-sommables et leurs applications. In: Analysis, Micrological Calculus and Relativistic Quantum Theory, volume 126 of Lecture Notes in Physics, pp. 178-199. Springer, 1980.

J.-P. Ramis. Séries Divergentes et Théorie Asymptotiques, volume 121 of Panoramas et synthèses. Soc. Math. France, Paris, 1993.

W.H. Ray. Advanced Process Control. McGraw-Hill, 1981.

R. Rothfuß. Anwendung der flachheitsbasierten Analyse und Regelung nichtlinearer Mehrgrößensysteme. Fortschr.-Ber. VDI Reihe 8 Nr. 664. VDI Verlag, Düsseldorf, 1997.

R. Rothfuß, U. Becker, and J. Rudolph. Controlling a solenoid valve - a distributed parameter approach. In: Proc. 14th Int. Symposium on Mathematical Theory of Networks and Systems (MTNS), Perpignan, France, 2000.

R. Rothfuß, J. Rudolph, and M. Zeitz. Flatness-based control of a nonlinear chemical reactor model. Automatica, 32:1433-1439, 1996. 
R. Rothfuß, J. Rudolph, and M. Zeitz. Flachheit: Ein neuer Zugang zur Steuerung und Regelung nichtlinearer Systeme. at-Automatisierungstechnik, 45:517-525, 1997.

P. Rouchon. Motion planning, equivalence, and infinite dimensional systems. Int. J. Appl. Math. Comp. Sc., 11:165-188, 2001.

P. Rouchon. Flatness-based control of oscillators. ZAMM, 85(6):411-421, 2005.

W. Rudin. Principles of mathematical analysis. McGraw-Hill, 3rd edition, 1976.

W. Rudin. Reelle und Komplexe Analysis. Oldenbourg Wissenschaftsverlag GmbH, 1999.

J. Rudolph. Flatness-based control by quasi-static feedback illustrated on a cascade of two chemical reactors. Int. J. Control, 73(2):115-131, 2000a.

J. Rudolph. Randsteuerung von Wärmetauschern mit örtlich verteilten Parametern: ein flachheitsbasierter Zugang. at-Automatisierungstechnik, 48:399-406, $2000 \mathrm{~b}$.

J. Rudolph. Beiträge zur flachheitsbasierten Folgeregelung linearer und nichtlinearer Systeme endlicher und unendlicher Dimension. Berichte aus der Steuerungs- und Regelungstechnik. Shaker-Verlag, 2003a.

J. Rudolph. Flatness Based Control of Distributed Parameter Systems. Berichte aus der Steuerungsund Regelungstechnik. Shaker-Verlag, 2003b.

J. Rudolph. Planning trajectories for a class of linear partial differential equations: an introduction. Sciences et Technologies de l'Automatique (e-sta), 1, 2004. Electronic Journal: http://www.esta.see.asso.fr.

J. Rudolph, J. Winkler, and F. Woittennek. Flatness Based Control of Distributed Parameter Systems: Examples and Computer Exercises from Various Technological Domains. Berichte aus der Steuerungs- und Regelungstechnik. Shaker-Verlag, 2003.

J. Rudolph and F. Woittennek. Flachheitsbasierte Randsteuerung elastischen Balken mit Piezoaktuatoren. at-Automatisierungstechnik, 50:412-421, 2002.

J. Rudolph and F. Woittennek. Motion Planning for Euler-Bernoulli Beams. In: H. Sira-Ramírez and G. Silva-Navarro (eds.), Algebraic Methods in Flatness, Signal Processing and State Estimation, chapter 8, pp. 131-148. Innovacion Editoral Lagares, Mexico, 2003.

D.L. Russell. Controllability and stabilizability theory for linear partial differential equations: Recent progress and open questions. SIAM Review, 20(4):639-739, 1978.

H. Sano and N. Kunimatsu. An Application of Inertial Manifold Theory to Boundary Stabilization of Semilinear Diffusion Systems. J. Math. Anal. Appl., 196:18-42, 1995.

D.A. Smith and W.F. Ford. Acceleration of linear and logarithmic convergence. SIAM J. Num. Anal., 16(2):223-240, 1979. 
A.D. Sokal. An improvement of Watson's theorem on Borel summability. J. Math. Phys., 21(2): 261-263, 1980.

M.E. Taylor. Partial Differential Equations I. Basic Theory. Springer-Verlag, New York, 1996.

R. Temam. Infinite-Dimensional Dynamical Systems in Mechanics and Physics. New York: SpringerVerlag, 1988.

M.O. Wagner, T. Meurer, and M. Zeitz. $K$-summable power series as a design tool for feedforward control of diffusion-convection-reaction systems. In: Proc. 6th IFAC Symposium "Nonlinear Control Systems" (NOLCOS 2004), pp. 149-154, Stuttgart, Germany, 2004.

G.N. Watson. A theory of asymptotic series. Phil. Trans. Roy. Soc. London, 211:279-313, 1912.

E.J. Weniger. Nonlinear sequence transformations for the acceleration of convergence and the summation of divergent series. Compt. Phys. Rep., 10:189-371, 1989.

E.J. Weniger. Verallgemeinerte Summationsprozesse als numerische Hilfsmittel für quantenmechanische und quantenchemische Rechnungen. Habilitation thesis (in German), Universität Regensburg, Germany, 1994. available at http://arxiv.org.

D.W. Widder. The Heat Equation. Academic Press, 1975.

F. Woittennek and J. Rudolph. Motion planning for a class of boundary controlled linear hyperbolic PDE's involving finite distributed delays. ESAIM: Control, Optimisation and Calculus of Variations, 9:419-435, 2003.

S. Wolfram. The Mathematica Book. Cambridge University Press, 4. edition, 1999.

J. Zinn-Justin. Quantum field theory and critical phenomena. International series of monographs on physics 77. Oxford : Clarendon Press, 1989.

E. Zuazua. Controllability of Partial Differential Equations, 2004. Manuscript. 


\section{Internal reports, student and diploma theses}

T. Meurer. Flatness-based feedback boundary control for an infinite dimensional system modeling a heat conduction rod. Internal report 2002-02, Institut für Systemdynamik und Regelungstechnik, Univ. Stuttgart, 2002.

T. Meurer. A modal analysis approach to flatness-based open-loop control of distributed parameter systems. Internal report 2003-02, Institut für Systemdynamik und Regelungstechnik, Univ. Stuttgart, 2003a.

T. Meurer. Mathematica packages for optimized code conversion, 2003b.

J. Becker. Flachheitsbasierte Folgeregelung von verteilten Reaktions-, Diffusions- und Wärmeleitsystemen. Studienarbeit, Institut für Systemdynamik und Regelungstechnik, Univ. Stuttgart, 2003.

M. Boldt. Flachheitsbasierte Folgeregelung von verteilten linearen Diffusions- und Wärmeleitsystemen. Studienarbeit, Institut für Systemdynamik und Regelungstechnik, Univ. Stuttgart, 2003.

A. Schaum. Steuerungsentwurf für Diffusions-Konvektions-Reaktionssysteme mittels Summationsmethoden. Studienarbeit, Institut für Systemdynamik und Regelungstechnik, Univ. Stuttgart, 2005.

M. Schenk. Vorsteuerung mit prädiktiver PI-Regelung für lineare und nichtlineare Erhaltungsgleichungen. Studienarbeit, Institut für Systemdynamik und Regelungstechnik, Univ. Stuttgart, 2003.

M.O. Wagner. Flachheitsbasierter Steuerungs- und Folgeregelungsentwurf für verteiltparametrische Systeme mittels formaler Potenzreihen. Diplomarbeit, Institut für Systemdynamik und Regelungstechnik, Univ. Stuttgart, 2003. Awarded with the "Studienpreis 2003" of the SEW-Eurodrive Foundation. 